\title{
Estimação do índice de memória em processos estocásticos com memória longa: \\ Uma abordagem via $\mathrm{ABC}$
}

\author{
Plinio Lucas Dias Andrade
}

\author{
TESE APRESENTADA \\ $\mathrm{AO}$ \\ Instituto DE MATEMÁticA E EstatísticA \\ DA \\ UniVERSIDADE DE SÃO PAUlo \\ PARA \\ OBTENÇÃO DO TÍTULO \\ $\mathrm{DE}$ \\ DOUTOR EM CIÊNCIAS

\begin{abstract}
Programa: Pós-Graduação em Estatística
Orientador: Prof. Dr. Carlos Alberto de Bragança Pereira

Coorientadora: Prof ${ }^{a}$. Dra ${ }^{a}$. Laura Letícia Ramos Rifo
\end{abstract}

Durante o desenvolvimento deste trabalho o autor recebeu auxílio financeiro da CAPES e do $\mathrm{CNPq}$

São Paulo, maio de 2016 


\section{Estimação do índice de memória em processos estocásticos com memória longa: Uma abordagem via ABC}

Esta versão da tese contém as correções e alterações sugeridas pela Comissão Julgadora durante a defesa da versão original do trabalho, realizada em 28/03/2016. Uma cópia da versão original está disponível no Instituto de Matemática e Estatística da Universidade de São Paulo.

Comissão Julgadora:

- Prof ${ }^{a}$. Dra ${ }^{a}$. Laura Letícia Ramos Rifo (coorientadora) - IMECC-UNICAMP

- Prof ${ }^{a}$. Dra . Florencia Graciela Leonardi - IME-USP

- Prof ${ }^{\mathrm{a}}$. Dr ${ }^{\mathrm{a}}$. Chang Chung Yu Dorea - UNB

- Prof. Dr. Paulo Regis Caron Ruffino - IMECC-UNICAMP

- Prof. Dr. Marcelo Sobottka - UFSC 


\section{Agradecimentos}

Agradeço primeiramente a Deus e a minha família por estarem sempre presentes. Sou grato especialmente ao meu irmão com quem compartilhei dificuldades e sucessos ao longo desses anos.

Agradeço também aos colegas de Doutorado com quem muito aprendi, em particular aos amigos Bruno Ramos, Eder Fonseca e Joelson Campos.

Sou muito grato ao Professor Carlinhos por sua paciência, disponibilidade e espírito motivador, bem como à Professora Laura Rifo, orientadora e amiga, por ter me guiado brilhantemente com muita paciência e dedicação rumo ao desfecho deste trabalho.

Finalmente, agradeço à CAPES e ao CNPq pelo apoio financeiro. 


\section{Resumo}

\section{ANDRADE, P. L. D. Estimação do índice de memória em processos estocásticos com me-}

mória longa: Uma abordagem via ABC. 2016. Tese (Doutorado) - Instituto de Matemática e Estatística, Universidade de São Paulo, São Paulo, 2016.

Neste trabalho propomos o uso de um método Bayesiano para estimar o parâmetro de memória de um processo estocástico com memória longa quando sua função de verossimilhança é intratável ou não está disponível. Esta abordagem fornece uma aproximação para a distribuição a posteriori sobre a memória e outros parâmetros e é baseada numa aplicação simples do método conhecido como computação Bayesiana aproximada (ABC). Alguns estimadores populares para o parâmetro de memória serão revisados e comparados com esta abordagem. O emprego de nossa proposta viabiliza a solução de problemas complexos sob o ponto de vista Bayesiano e, embora aproximativa, possui um desempenho muito satisfatório quando comparada com métodos clássicos.

Palavras-chave: processo estocástico com memória longa, inferência Bayesiana, computação Bayesiana aproximada, ABC. 


\section{Abstract}

ANDRADE, P. L. D. Estimation of the memory index of stochastic processes with long memory: An ABC approach. 2016. Thesis (PhD) - Instituto de Matemática e Estatística, Universidade de São Paulo, São Paulo, 2016.

In this work we propose the use of a Bayesian method for estimating the memory parameter of a stochastic process with long-memory when its likelihood function is intractable or unavailable. Such approach provides an approximation for the posterior distribution on the memory and other parameters and it is based on a simple application of the so-called approximate Bayesian computation $(\mathrm{ABC})$. Some popular existing estimators for the memory parameter are reviewed and compared to this method. The use of our proposal allows for the solution of complex problems under a Bayesian point of view and this proposal, although approximative, has a satisfactory performance when compared to classical methods.

Keywords: long memory stochastic process, Bayesian inference, approximate Bayesian computation, ABC. 


\section{Sumário}

$\begin{array}{ll}\text { Lista de Abreviaturas } & \text { ix }\end{array}$

Lista de Figuras

Lista de Tabelas $\quad$ xvii

1 Introdução $\quad 1$

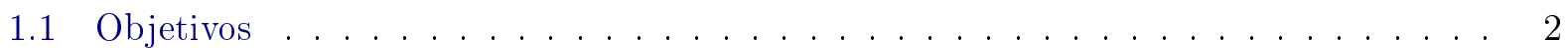

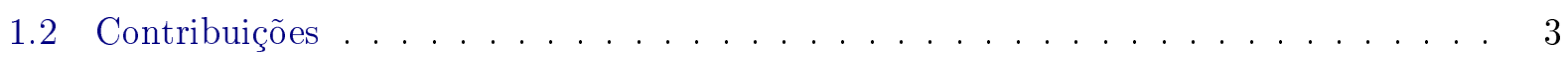

1.3 Organização do Trabalho . . . . . . . . . . . . . . . . . . . . . . 3

2 Processos estocásticos com memória longa $\quad 5$

2.1 Motivação e definições . . . . . . . . . . . . . . . . . . . . 5

2.2 Alguns processos com memória longa . . . . . . . . . . . . . 8

$2.2 .1 \quad$ Processos autossimilares . . . . . . . . . . . . . . . . . . 8

2.2 .2 Processos de Hermite . . . . . . . . . . . . . . . . . . . . . . . . . 10

2.2.3 Movimento estável fracionário . . . . . . . . . . . . . . . . . . . . . . . . . 14

2.2.4 Processo gama modulado generalizado . . . . . . . . . . . . . . . 18

3 Estimadores clássicos para o índice de memória $\quad 23$

3.1 Análise do variograma . . . . . . . . . . . . . . . . . . . 24

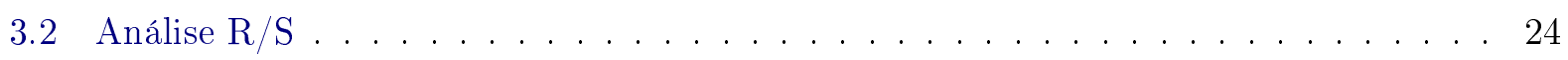

3.3 Análise local e global do periodograma . . . . . . . . . . . . . . . 25

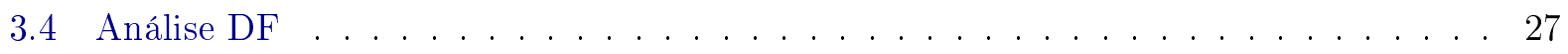

3.5 Métodos baseados no estimador de Whittle . . . . . . . . . . . . . . . 28

3.6 Método de ondaletas . . . . . . . . . . . . . . . . . . . . . . 29

3.7 Variação-p . . . . . . . . . . . . . . . . . . . . . . . 30

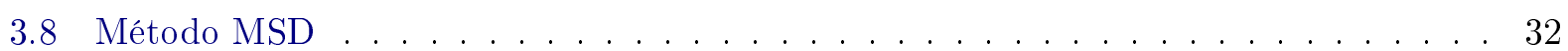

4 Computação Bayesiana Aproximada $\quad 33$

4.1 Sobre a escolha de estatísticas resumo . . . . . . . . . . . . . . . . . 44

4.1 .1 Critério de mínima entropia . . . . . . . . . . . . . . . . . . 44

4.1 .2 Critério de $\epsilon$-suficiência . . . . . . . . . . . . . . . . . . 45

$4.1 .3 \mathrm{ABC}$ semiautomático . . . . . . . . . . . . . . . . . . . . 47

4.2 Pós-processamento da amostra $\mathrm{ABC} \ldots \ldots \ldots \ldots$. . . . . . . . . . . . . 48

4.3 Uma nota sobre a escolha de modelos e testes de hipóteses através do ABC . . . . . 52 
5 Aplicações e Conclusões $\quad \mathbf{6 1}$

5.1 Descrição dos resultados . . . . . . . . . . . . . . . . . 61

5.2 Ruído Gaussiano Fracionário . . . . . . . . . . . . . . . . . . 61

5.2.1 Aplicação: Níveis mínimos anuais do rio Nilo . . . . . . . . . . . . . . . 63

5.3 Incrementos do processo de Rosenblatt . . . . . . . . . . . . . . . . . . 65

5.3.1 Aplicação: Vazão média mensal dos rios Mosa e Vístula . . . . . . . . . . . . 67

5.4 Processo binário com memória longa . . . . . . . . . . . . . . . . . . . . 70

5.5 Movimento estável fracionário . . . . . . . . . . . . . . . . . . . . . 72

5.5.1 Aplicação: Erupções solares . . . . . . . . . . . . . . . . . . . . 75

5.6 Processo gama modulado generalizado . . . . . . . . . . . . . . . . . . 78

5.6.1 Aplicação: Retornos do índice S\&P500 . . . . . . . . . . . . . . . . . . 82

5.7 Considerações Finais . . . . . . . . . . . . . . . . . . . . . . . . 83

5.8 Sugestões para Pesquisas Futuras . . . . . . . . . . . . . . . . . . . . 85

$\begin{array}{lll}\text { A } & \text { Tabelas de Resultados } & \mathbf{8 7}\end{array}$

$\begin{array}{ll}\text { Referências Bibliográficas } & 93\end{array}$ 


\title{
Lista de Abreviaturas
}

\author{
ABC Computação Bayesiana aproximada (Approximate Bayesian computation) \\ DFA Detrended fluctuation analysis \\ dp Desvio padrão \\ EQM Erro quadrático médio \\ $\mathrm{fBm} \quad$ Movimento Browniano fracionário (Fractional Brownian motion) \\ fGn Ruído Gaussiano fracionário (Fractional Gaussian noise) \\ fSm Movimento estável fracionário (Fractional stable motion) \\ fSn Ruído estável fracionário (Fractional stable noise) \\ MCMC Monte Carlo via cadeias de Markov (Markov chain Monte Carlo) \\ PML Processo com memória longa \\ PMC Processo com memória curta \\ Rn Ruído Rosenblatt (Rosenblatt noise) \\ SAABC ABC semiautomático (Semiautomatic ABC)
}




\section{Lista de Figuras}

1.1 Trajetórias do ruído Gaussiano fracionário para diferentes intensidades de memória: sem memória (primeira gráfico), memória longa com intensidade intermediária (segunda gráfico) e memória longa com alta intensidade (terceiro gráfico). . . . . . . 2

2.1 Triângulo de Sierpenski. . . . . . . . . . . . . . . . . . . . . . . . 8

2.2 Níveis mínimos anuais do rio Nilo e intervalos de previsão obtidos utilizando-se dois modelos Gaussianos: Um com memória longa (ARIMA fracionário) e um com memória curta (autoregressivo de ordem 2) . . . . . . . . . . . . . . 10

2.3 Trajetórias do processo de Hermite de ordem 1 (acima e a esquerda) e ordem 2 (abaixo e a esquerda) com parâmetro de memória $H=0.7$ e suas respectivas séries de incrementos $($ coluna direita) . . . . . . . . . . . . . . . . . . . 12

2.4 Funções densidade (esquerda) e distribuição acumulada (direita) da distribuição $\alpha$ estável com parâmetros $\beta=0, \gamma=1, \delta=0$ e $\alpha$ variando em $\{2.0,1.5,1.0,0.5\}$. . . 15

2.5 Funções densidade (esquerda) e distribuição acumulada (direita) da distribuição $\alpha$ estável com parâmetros $\alpha=0.5, \gamma=1, \delta=0$ e $\beta$ variando em $\{0.00,0.25,0.50,1.00\} .16$

2.6 Trajetória amostral do fBm (acima) e do fSm (abaixo) com parâmetro de autossimilaridade $H=0.8 \ldots \ldots \ldots \ldots \ldots \ldots$. . . . . . . . . . . . 17

2.7 Trajetória amostral do fGn (acima) e do fSn (abaixo) com parâmetro de autossimi-

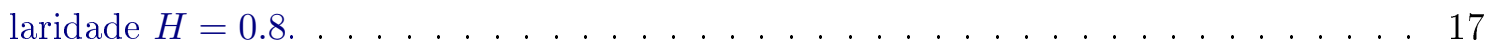

2.8 Trajetórias amostrais do processo gama-modulado generalizado (GGMp) para diferentes valores de $\beta \ldots \ldots \ldots \ldots \ldots$. . . . . . . . . . . . . . 19

2.9 Trajetórias amostrais do processo de incrementos do processo gama-modulado gene-

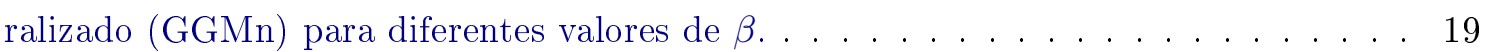

2.10 Distribuição amostral de $\operatorname{Var}\left[\Delta\left({ }^{\beta} X_{t}\right)\right]$ para $t=1,2, \ldots, 100$ obtida através de 1000 trajetórias simuladas do GGMn para diferentes valores de $\beta \ldots \ldots$. . . . . . . . 20

3.1 Variograma para séries simuladas do fGn com $H=0.5$ (esquerda) e $H=0.8$ (direita). A linha cheia foi obtida por mínimos quadrados, enquanto a linha tracejada corresponde a inclinação -1 (memória curta) . . . . . . . . . . . . . . . . 25

3.2 Poxplot para séries simuladas do fGn com $H=0.5$ (esquerda) e $H=0.8$ (direita). A linha cheia foi obtida por mínimos quadrados, enquanto a linha tracejada corresponde a inclinação 0.5 (memória curta) . . . . . . . . . . . . . . . . . . 25

3.3 Periodograma para séries simuladas do fGn com $H=0.5$ (esquerda) e $H=0.8$ (direita). A linha cheia foi obtida por mínimos quadrados, enquanto a linha tracejada corresponde a inclinação 0 (memória curta) . . . . . . . . . . . . . . . . 26 
3.4 Gráficos obtidos pela DFA para séries simuladas do fGn com $H=0.5$ (esquerda) e $H=0.8$ (direita). A linha cheia foi obtida por mínimos quadrados, enquanto a linha tracejada corresponde a inclinação 1 (memória curta) . . . . . . . . . . . . . .

4.1 Estudo da média, mediana e desvio-padrão como estatísticas resumo para o parâmetro de localização $\delta$ da distribuição $S(1.5,0.3,1, \delta) \ldots \ldots \ldots \ldots$

4.2 Histograma dos valores de $\delta$ obtidos pelo algoritmo D para uma amostra de tamanho 100 da distribuição $S(1.5,0.3,1, \delta=2)$. A curva tracejada representa a função de densidade a priori que neste caso é $N(0,25) \ldots \ldots \ldots \ldots \ldots$

4.3 Boxplots das médias a posteriori obtidas para $\delta$ usando o algoritmo D em 100 simulações de Monte Carlo. Os números $1-4$ no eixo horizontal indicam as abordagens (i) $-(i v)$.

4.4 Boxplots das médias a posteriori obtidas para $\delta$ usando o algoritmo D em 100 simulações de Monte Carlo. Os números $1-5$ no eixo horizontal indicam cada uma das 5 prioris assumidas para $\delta \ldots \ldots \ldots \ldots \ldots \ldots \ldots \ldots \ldots \ldots \ldots \ldots \ldots \ldots \ldots \ldots \ldots \ldots$

4.5 Traço dos valores de $\delta$ obtidos pelo algoritmo E para uma amostra de tamanho 100 da distribuição $S(1.5,0.3,1, \delta=2)$ e histograma desses valores após exclusão dos 5000 primeiros. A curva tracejada representa a função de densidade a priori que neste caso

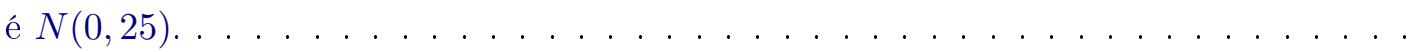

4.6 Distribuição a posteriori para $\theta=\left(\theta_{1}, \theta_{2}\right)$. O triângulo representa os valores que os parâmetros podem assumir.

4.7 Dispersão dos pontos aceitos pelo $\mathrm{ABC}$ (algoritmo $\mathrm{C}$ ) variando os valores de $\varepsilon$ de modo a aceitar $10 \%$ (esquerda), $1 \%$ (centro) e $0.1 \%$ (direita) das simulações. Cada gráfico possui curvas de nível da distribuição a posteriori teórica e os triângulos tracejados representam a distribuição a priori. . . . . . . . . . . . . .

4.8 Dispersão dos pontos aceitos pelo ABC (algoritmo D) utilizando as duas primeiras autocovariâncias como estatísticas resumo. Os valores de $\varepsilon$ foram tomados de modo a aceitar 10\% (esquerda), 1\% (centro) e 0.1\% (direita) das simulações. Cada gráfico possui curvas de nível da distribuição a posteriori teórica e os triângulos tracejados representam a distribuição a priori. . . . . . . . . . . . . . . .

4.9 Distribuições a posteriori marginais de $\theta=\left(\theta_{1}, \theta_{2}\right)$ obtidas pelo algoritmo D. A curva sólida representa a distribuição teórica, enquanto as curvas obtidas através das amostras 1, 2 e 3 correspondem aos valores de $\varepsilon$ considerados no algoritmo. . . . . .

4.10 Distribuições a posteriori marginais de $\theta=\left(\theta_{1}, \theta_{2}\right)$ obtidas pelo algoritmo D. A curva sólida representa a distribuição teórica, enquanto as demais curvas correspondem as amostras obtidas sem pós processamento (s/ correção), com pós processamento de Beaumont et al. (2002) (correção 1) e de Blum e François (2010) (correção 2). . . .

4.11 Níveis das densidades conjuntas para $(A, B)$ (esquerda) e $(g, k)$ (direita) utilizando o ABC proposto por Allingham et al. (2009) (gráficos superiores) e o método ABC semiautomático de Fearnhead e Prangle (2012) (gráficos inferiores) para uma amostra de tamanho 1000 com valor nominal de $\theta$ igual a $\theta_{0}=(3,1,2,0.5)$. O " $\times$ " demarca

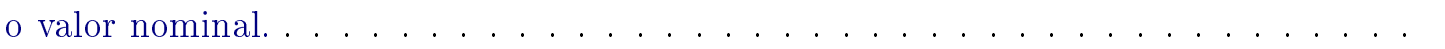

4.12 Gráficos da função de distribuição (esquerda) e função de densidade (direita) da distribuição de Rosenblatt para alguns valores de $D$. . . . . . . . . . . . . . . 56 
4.13 Distribuição amostral da média a posteriori para $D$ obtidas pelo algoritmo D. Cada coluna corresponde a um valor nominal utilizado nas simulações. . . . . . . . . . . 57

4.14 Distribuição a posteriori para $D$ obtido pelo ABC semiautomático para uma amostra de tamanho 1000 da distribuição de Rosenblatt quando: (a) $D=0.1$, (b) $D=0.2$, (c) $D=0.3$ e (d) $D=0.4$. . . . . . . . . . . . . . . . . . . . . . . . . . . 58

4.15 e-valores obtidos numericamente com respeito às hipóteses $D_{0}=0$ (esquerda) e $D_{0}=0.5$ (direita). ........................ 59

5.1 Boxplots dos desvios relativos com respeito ao valor nominal de $H \in\{0.2,0.5,0.7,0.9\}$ em 200 simulações do processo fGn segundo cada método de estimação. Cada número no eixo horizontal corresponde ao respectivo método de estimação enumerado anteriormente. . . . . . . . . . . . . . . . . . . . . . . . 64

5.2 Níveis mínimos anuais do rio Nilo entre os anos 622 e 1284 . . . . . . . . . . . . . . . 64

5.3 (a) Gráfico dos quantís teóricos da distribuição normal padrão contra os quantís empíricos da série observada padronizada. (b) Histograma da série observada e curva da densidade normal ajustada aos dados por máxima verossimilhança. (c) Análise $\mathrm{R} / \mathrm{S}$ da série observada. . . . . . . . . . . . . . . . . . . . . . 65

5.4 Posterioris aproximadas para H: (linha cheia) obtida pelo algoritmo D e (linha tracejada) ABC semiautomático. A linha pontilhada indica a distribuição a priori. . . . 66

5.5 Boxplots dos desvios relativos com respeito ao valor nominal de $H \in\{0.55,0.75,0.85,0.95\}$ em 200 simulações do processo Rn segundo cada método de estimação. Cada número no eixo horizontal corresponde ao respectivo método de estimação enumerado anteriormente. . . . . . . . . . . . . . . . . . . . . . . 67

5.6 Vazão média mensal dos rios Mosa e Vístula. . . . . . . . . . . . . . . . . . . . . 68

5.7 (a) Série do rio Mosa livre de sazonalidade. (b) Gráfico dos quantís teóricos da distribuição normal padrão contra os quantís empíricos da série observada padronizada.

(c) Histograma da série observada (dessazonalizada) e curva da densidade normal ajustada aos dados por máxima verossimilhança. (d) Análise R/S da série observada (dessazonalizada)............................ . 68

5.8 (a) Série do rio Vístula livre de sazonalidade. (b) Gráfico dos quantís teóricos da distribuição normal padrão contra os quantís empíricos da série observada padronizada.

(c) Histograma da série observada (dessazonalizada) e curva da densidade normal ajustada aos dados por máxima verossimilhança. (d) Análise R/S da série observada (dessazonalizada)......................... 69

5.9 Posterioris aproximadas para o parâmetro $H$ obtidas pelos métodos ABC para os dados dos rios Mosa e Vístula. . . . . . . . . . . . . . . . . . . . 70

5.10 Histograma das séries dessazonalizadas e padronizadas dos rios Mosa (a) e Vístula (b) com curva da densidade da distribuição de Rosenblatt com parâmetro $\hat{D}=2-2 \hat{H}$, onde $\hat{H}$ são, respectivamente iguais a 0.8089 e 0.8297 . . . . . . . . . . . . . . . . . 70

5.11 Trajetórias amostrais de comprimento 100 do processo binário com diferentes índices de memória. . . . . . . . . . . . . . . . . . . . . . . . . . 71 
5.12 Boxplots dos desvios relativos com respeito ao valor nominal de $H \in\{0.2,0.5,0.7,0.9\}$ em 200 simulações do processo binário com memória longa segundo cada método de estimação. Cada número no eixo horizontal corresponde ao respectivo método de estimação enumerado anteriormente. . . . . . . . . . . . . . . . .

5.13 Boxplots dos desvios relativos com respeito ao valor nominal de $d=H-\frac{1}{\alpha}$, com $H=0.6$ e $\alpha \in\{1.7,1.8,1.9\}$ (divididos pelas linhas verticais pontilhadas). Cada coluna corresponde a um método (etiquetado no eixo $0 x$ ). . . . . . . . . . .

5.14 Boxplots dos desvios relativos com respeito ao valor nominal de $d=H-\frac{1}{\alpha}$, com $H=0.7$ e $\alpha \in\{1.7,1.8,1.9\}$ (divididos pelas linhas verticais pontilhadas). Cada coluna corresponde a um método (etiquetado no eixo $0 x) \ldots \ldots \ldots \ldots$

5.15 Boxplots dos desvios relativos com respeito ao valor nominal de $d=H-\frac{1}{\alpha}$, com $H=0.8$ e $\alpha \in\{1.7,1.8,1.9\}$ (divididos pelas linhas verticais pontilhadas). Cada coluna corresponde a um método (etiquetado no eixo $0 x$ ) . . . . . . . . . .

5.16 Boxplots dos desvios relativos com respeito ao valor nominal de $d=H-\frac{1}{\alpha}$, com $H=0.9$ e $\alpha \in\{1.7,1.8,1.9\}$ (divididos pelas linhas verticais pontilhadas). Cada coluna corresponde a um método (etiquetado no eixo $0 x) \ldots \ldots \ldots \ldots$

5.17 Emissões energéticas de erupções solares obtidas pelo satélite GOES no período de

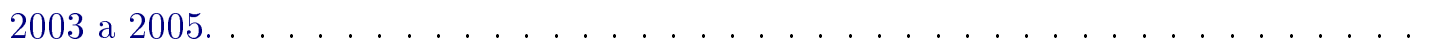

5.18 (a) Histograma da série observada de erupções solares e curva da densidade estável ajustada. (b) Gráfico dos quantís teóricos da distribuição estável ajustada aos dados contra os quantís empíricos da série observada. (c) Análise $\mathrm{R} / \mathrm{S}$ aplicada à série observada. (d) Posteriori aproximada para $\alpha$ obtida pelo método ABC semiautomático. 77

5.19 Posteriori aproximada para $H$ obtida pelo método ABC 2 apresentado na seção 5.5. . 78

5.20 Variância em blocos para séries simuladas do GGMn para diferentes valores de $\beta$ em escala logaritmica. A linha cheia corresponde à reta ajustada aos pontos por mínimos quadrados, enquanto a linha tracejada corresponde à linha teórica cuja inclinação é

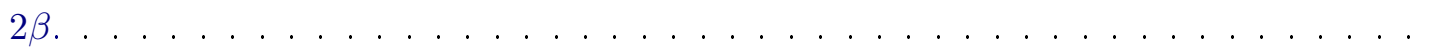

5.21 Gráficos dos parâmetros $(\beta, \mu, \sigma)$ simulados da priori plotados contra os valores de suas respectivas estatísticas resumo calculadas em 10000 simulações do processo GGMn. 80

5.22 Distribuição amostral da média aproximada a posteriori para $\beta$ em 200 simulações de Monte Carlo através do algoritmo D para diferentes valores de $\beta . \quad \ldots . .81$

5.23 Boxplots dos $e$-valores obtidos a partir da amostra aproximada a posteriori em 200 simulações de Monte Carlo para diferentes valores de $\beta$ : (a) $\beta_{0}=-0.5$; (b) $\beta_{0}=0 . \quad$. 81

5.24 Gráficos dos parâmetros $(\mu, \sigma)$ simulados da priori plotados contra os valores de suas respectivas estatísticas resumo obtidas pelo método SAABC . . . . . . . . . . 82

5.25 Distribuição amostral da média aproximada a posteriori para $\mu$ (esquerda) e $\sigma$ (direita), obtidas em 200 simulações de Monte Carlo variando $\beta$. . . . . . . . . . .

5.26 Níveis das distribuições amostrais conjuntas de $(\mu, \sigma)$ obtidas pelo método SAABC em 200 simulações de Monte Carlo para diferentes valores de $\beta$. . . . . . . . . .

5.27 (a) log-retornos dos valores de fechamento do índice S\&P500 no período de novembro de 2005 a março de 2009. (b) Variância em blocos da série observada e reta ajustada por mínimos quadrados. . . . . . . . . . . . . . . . . . . . . . 
5.28 Distribuições a posteriori aproximadas obtidas para os parâmetros $(\beta, \mu, \sigma)$ para os dados do índice S\&P500. 


\section{Lista de Tabelas}

4.1 Estudo do impacto da escolha de $\varepsilon$ na inferência sobre $\delta$ usando o algoritmo D. . . . 36

4.2 Média e desvio-padrão das estimativas (média a posteriori) obtidas para $\delta$ usando o algoritmo D em 100 simulações de Monte Carlo segundo as abordagens $(i)-(i v)$. . 38

4.3 Média e devio-padrão das estimativas (média a posteriori) obtidas para $\delta$ usando o algoritmo D em 100 simulações de Monte Carlo utilizando diferentes distribuições a priori para $\delta \ldots \ldots \ldots \ldots \ldots \ldots \ldots \ldots \ldots \ldots \ldots \ldots \ldots \ldots \ldots \ldots \ldots \ldots \ldots$

4.4 Média e devio-padrão das estimativas (média a posteriori) obtidas para $\delta$ usando o algoritmo E em 100 simulações de Monte Carlo utilizando as prioris $N(0,25)$ e

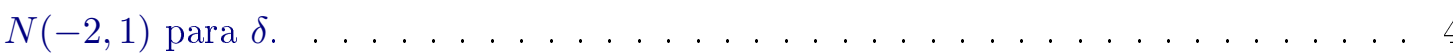

4.5 Erro quadrático médio das estimativas baseadas em 50 simulações de Monte Carlo da distribuição $g$-and- $k$ de tamanho $n=10^{4}$ e parâmetro nominal igual a $\theta_{0}=$ $(3,1,2,0.5)$, segundo cada abordagem. . . . . . . . . . . . . 52

4.6 Estimativas para as probabilidades a posteriori de cada modelo obtidas através do algoritmo $\mathrm{G}$ considerando diferentes valores para $\varepsilon$. O valor $q_{p \%}(d)$ indica o número de simulações aceitas. A última linha indica os valores verdadeiros de $\mathbb{P}(\mathcal{M}=m \mid \mathcal{D})$ obtidos por integração numérica. . . . . . . . . . . . . . . . . 54

4.7 Primeiros 10 valores de $\lambda_{n}(D)$ e $\lambda_{500}(D)$ para diferentes valores de $D$ obtidos nume-

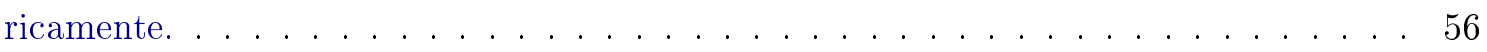

4.8 Média e desvio padrão amostral das estimativas para $D$ em 100 simulações de Monte Carlo para cada métrica considerada. . . . . . . . . . . . . . . 58

4.9 Erro quadrático médio (EQM) das estimativas obtidas (média a posteriori aproximada) em 100 simulações de Monte Carlo segundo diferentes aplicações do método

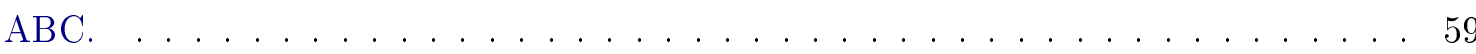

5.1 Estimativas de $H$ para a série de níveis mínimos anuais do rio Nilo obtidas por

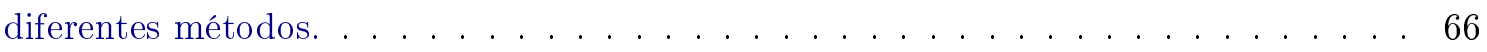

5.2 Estimativas de $H$ para as séries de vazão média mensal dos rios Mosa e Vístula por diferentes métodos. . . . . . . . . . . . . . . . . 71

5.3 Estimativas de $H$ obtidas pelos métodos de estimação apresentados na seção 5.5. 77

5.4 Resultados obtidos em 200 simulações de Monte Carlo utilizando-se os métodos ABCMCMC de Andrade et al. (2015) e SAABC. . . . . . . . . . . . . . . . 83

A.1 Resultados obtidos para $H$ através de diferentes métodos em 200 simulações de Monte Carlo do ruído Gaussiano fracionário $(\mathrm{fGn}) \ldots \ldots \ldots$. . . . . . . . 87 
A.2 Resultados obtidos para $H$ através de diferentes métodos em 200 simulações de Monte Carlo dos incrementos do processo de Rosenblatt (Rn) . . . . . . . . . . . . . . 88

A.3 Resultados obtidos para $H$ através de diferentes métodos em 200 simulações de Monte Carlo do processo binário com memória longa. . . . . . . . . . . . . . . . . . . . . 89

A.4 Resultados obtidos para $\alpha$ através dos métodos de Koutrouvelis (1980) e ABC 2 em 200 simulações de Monte Carlo do ruído estável fracionário (fSn) . . . . . . . . . . . . 90

A.5 Resultados obtidos para $H$ através de diferentes métodos em 200 simulações de Monte Carlo do ruído estável fracionário $(\mathrm{fSn}) . \ldots \ldots$. . . . . . . . . . . . 91

A.6 Resultados obtidos para $(\beta, \mu, \sigma)$ através do método ABC semiautomático em 200 simulações de Monte Carlo dos incrementos do processo gama modulado generalizado,

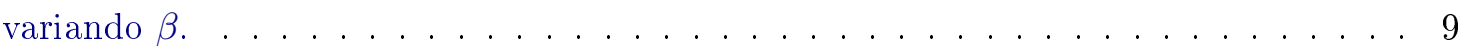




\section{Capítulo 1}

\section{Introdução}

A importância do conceito de dependência de longo alcance ou memória longa é refletida na quantidade de trabalhos relacionados a esse assunto nas mais diversas áreas, como em finanças (Lo, 1997), econometria (Robinson, 2003), modelagem de tráfego da internet (Karagiannis et al., 2004), linguística (Alvarez-Lacalle et al., 2006), sequenciamento de DNA (Karmeshu e Krishnamachari, 2004), estudos climáticos (Varotsos e Kirk-Davidoff, 2006) e especialmente em hidrologia (Painter, 1998). Em geral, tais trabalhos buscam resolver problemas como: detectar a presença de memória longa nos dados, teoremas limite sob a hipótese de dependência de longo alcance, simulação de processos com memória longa para emular certos fenômenos, estimação de parâmetros de memória longa, etc.

A noção de memória longa tem a ver com memória em um processo estocástico. Memória é, por definição, algo que perdura. O requerimento de que a memória deve ser "longa" que é especial. Qual a importância de que em um modelo a memória é "um pouco mais longa" que em outro modelo? (Samorodnitsky, 2007)

O fenômeno de memória longa é conhecido muito antes do desenvolvimento de modelos estocásticos adequados a esta característica. Pesquisadores de diversas áreas da aplicação estatística observaram empiricamente que as correlações entre observações que estão distantes (no tempo ou espaço) decaem mais lentamente do que o esperado em dados independentes ou dados que seguem modelos Markovianos ou ARMA (autoregressivo e de médias móveis). Harold Edwin Hurst, um famoso hidrólogo britânico, foi um dos pioneiros a observar empiricamente este fenômeno em sua tentativa de regularizar o fluxo do rio Nilo, conhecido por suas características bíblicas de longos períodos de baixo nível, seguido de longos períodos de cheias (Hurst, 1951). No entanto, uma resposta formal à pergunta acima só foi dada por Mandelbroot e seus colaboradores em uma série de trabalhos que foram motivados pelas descobertas de Hurst, trabalhos estes que viabilizaram o desenvolvimento da base matemática do movimento Browniano fracionário, cujo processo de incrementos, conhecido como ruído Gaussiano fracionário, é frequentemente utilizado como modelo para dados que apresentam memória longa (Mandelbrot e Van Ness, 1968; Mandelbrot e Wallis, 1968, 1969a,b,c).

A partir da observação de séries do ruído Gaussiano fracionário é que podemos ter uma idéia do que significa um modelo ter memória "mais longa" do que outro. A Figura 1.1 apresenta três trajetórias com diferentes intensidades de memória. Todas as séries foram simuladas de um processo estacionário. No caso i.i.d. (primeiro gráfico) a série oscila em torno de 0 sugerindo que o processo adjacente é estacionário, enquanto no gráfico onde a intensidade de memória é muito maior (terceiro gráfico), a série permanece por longos períodos acima e abaixo do nível 0 . Este fenômeno é conhecido como efeito Hurst em homenagem ao hidrólogo de mesmo nome. Outra característica é que se observarmos períodos curtos de tempo, é possível notar tendências locais nas séries com memória longa o que pode sugerir, se a série for observada apenas nesse período, que o processo gerador da série não seja estacionário. 

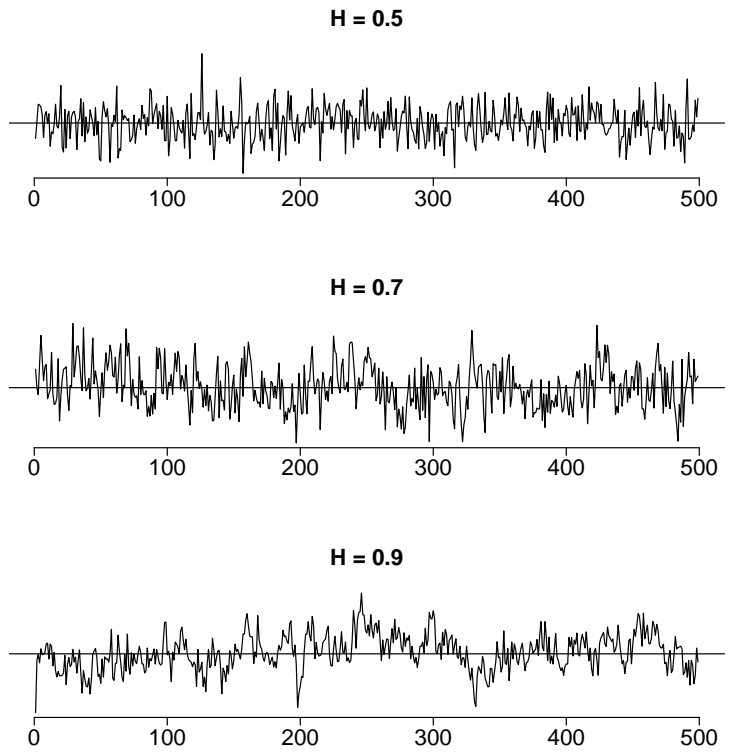

Figura 1.1: Trajetórias do ruído Gaussiano fracionário para diferentes intensidades de memória: sem memória (primeira gráfico), memória longa com intensidade intermediária (segunda gráfico) e memória longa com alta intensidade (terceiro gráfico).

Neste contexto, os processos Markovianos e grande parte dos modelos propostos na área de séries temporais não possuem memória longa. Embora exista uma estrutura de dependência definida para esses modelos, essa dependência é dita de curto alcance ou que o processo possui memória curta. $\mathrm{O}$ emprego desses modelos em séries que exibem memória longa não é indicada pois uma quantidade muito grande de parâmetros deve ser estimada. Além disso, esses modelos tendem a subestimar intervalos de previsão de observações futuras.

Em geral, estimar o parâmetro de memória longa não é uma tarefa simples. Processos Gaussianos com memória longa foram extensivamente estudados e muitas técnicas de estimação foram desenvolvidas. Uma compilação de vários resultados pode ser obtida em Beran (1994). Mesmo no caso de processos Gaussianos, a estimação do parâmetro de memória longa nem sempre é simples, pois complicações computacionais surgem quando a intensidade de memória cresce e métodos clássicos, como máxima verossimilhança, precisam ser adaptados. Quando a suposição de normalidade não é razoável, a estimação da memória é feita a partir de técnicas heurísticas ou semiparamétricas, muitas delas construídas com base em processos Gaussianos. Muitas dessas abordagens continuam consistentes, mas com complicadas distribuições limite.

Um agravante que surge na análise da memória de processos com memória longa é que grande parte destes não possuem forma analítica para suas distribuições finito dimensionais e, consequentemente, para suas funções de verossimilhança, fazendo com que técnicas baseadas no cálculo da verossimilhança, como métodos Bayesianos e de máxima verossimilhança, não possam ser utilizadas.

\section{$1.1 \quad$ Objetivos}

Neste trabalho propomos o uso de um método Bayesiano para estimar o parâmetro de memória para alguns processos estocásticos com memória longa. Para a maioria desses processos não é possível obter a função de verossimilhança para os parâmetros e, quando esta função está disponível, ela é intratável analiticamente, de modo que métodos numéricos baseados em verossimilhança, como Monte Carlo via cadeias de Markov, não podem ser aplicados. Por este motivo, propomos um método conhecido como computação Bayesiana aproximada ou simplesmente ABC (approximate Bayesian computation), método este que não necessita do cálculo da função de verossimilhança, bastando apenas que seja possível simular dados do modelo em estudo. Além disso, demonstramos através 
de dados sintéticos e reais, que a abordagem proposta neste trabalho produz resultados razoáveis quando comparados com os principais estimadores clássicos utilizados na literatura.

Em contraste com as abordagens clássicas, existem poucos trabalhos Bayesianos voltados ao estudo de processos estocásticos com memória longa, dado as dificuldades mencionadas anteriormente. Entre eles destacamos os trabalhos de Qu (2005), Holan et al. (2009) e Benmehdi et al. (2011) que abordam modelos Gaussianos semiparamétricos. Nosso objetivo é fornecer uma ferramenta que possa ser utilizada em processos mais gerais pois, embora o método aqui apresentado seja aplicado apenas em alguns processos, a idéia em si pode ser utilizada, com as devidas modificações, em qualquer modelo que se deseja estudar, desde que seja possível obter simulações do mesmo.

\subsection{Contribuições}

As principais contribuições deste trabalho são as seguintes:

- Chamar a atenção para a possibilidade de se estudar o parâmetro de memória longa de uma forma Bayesiana em processos não Gaussianos mais gerais e que não possuam função de verossimilhança tratável analiticamente.

- Fornecer uma nova abordagem para estimar o parâmetro de memória em alguns processos conhecidos e que esta seja competitiva com as abordagens clássicas mais utilizadas.

- Preencher a lacuna formada pelos poucos trabalhos Bayesianos na área de processos estocásticos com memória longa.

- Apresentar, como uma contribuição adicional, uma revisão dos principais avanços dos métodos ABC desenvolvidos ao longo dos últimos anos, bem como uma aplicação original deste método em um problema de estimação para a distribuição de Rosenblatt. Tal distribuição aparece frequentemente no contexto de processos com memória longa e não há na literatura, até o presente momento, uma abordagem inferencial para essa complicada distribuição.

\subsection{Organização do Trabalho}

Este trabalho está organizado da seguinte maneira: No Capítulo 2, definimos o conceito de memória longa e apresentamos uma revisão bibliográfica sobre os processos estocásticos que serão utilizados em nosso estudo. No Capítulo 3 apresentamos os principais estimadores clássicos encontrados na literatura e que serão utilizados para verificar a eficácia da abordagem proposta. O Capítulo 4 fornece uma revisão dos principais avanços da computação Bayesiana aproximada com diversos exemplos que ajudam a entender o funcionamento desse método. Neste mesmo capítulo, apresentamos um exemplo original onde o método $\mathrm{ABC}$ é utilizado para fazer inferência sobre a distribuição de Rosenblatt, uma importante distribuição que surge no contexto de processos com memória longa.

Finalmente, no Capítulo 5, apresentamos o modo como o método ABC é aplicado em cada processo estocástico considerado e validamos a sua eficácia através de dados simulados e reais. Os resultados obtidos por nossa abordagem são comparados com os resultados obtidos pelas principais abordagens clássicas quando disponíveis.

Algumas tabelas contendo resultados das simulações serão dadas no Apêndice A. 


\section{Capítulo 2}

\section{Processos estocásticos com memória longa}

O conceito de memória longa (dependência de longo alcance ou dependência de longo termo) pode ser definido de diversas formas a partir de diferentes pontos de vista. Beran et al. (2013) apresentam as definições mais utilizadas, enquanto Samorodnitsky (2007) apresenta uma série de definições mais sofisticadas voltadas para diferentes teorias como, por exemplo, teoria ergódica ou teoria de grandes desvios.

Neste capítulo vamos apresentar as definições mais utilizadas na literatura e que se ajustam melhor nos processos que iremos estudar. Essas definições são voltadas para processos estacionários e/ou autossimilares. Em seguida definiremos os processos estocásticos ${ }^{1}$ com memória longa que serão utilizados como modelos para nossa abordagem.

\subsection{Motivação e definições}

Uma forma de entender a definição de dependência de longo alcance é dada em Beran (1994). Vamos apresentá-la resumidamente aqui: Seja $X_{1}, \ldots, X_{n}$ uma amostra aleatória com $\mathrm{E}\left(X_{i}\right)=\mu$ e $\operatorname{Var}\left(X_{i}\right)=\sigma^{2}$ e suponha que o interesse seja calcular a variância de $\bar{X}_{n}=\frac{1}{n} \sum_{i=1}^{n} X_{i}$ para, por exemplo, obter um intervalo de confiança para $\mu$. Neste caso, temos que

$$
\operatorname{Var}\left(\bar{X}_{n}\right)=\sigma^{2} n^{-1}
$$

isto é, a variância da média amostral tende a zero como função de $n$.

Vamos agora considerar que exista dependência entre $X_{1}, \ldots, X_{n}$ e que essa dependência seja modelada por um processo $\mathrm{AR}(1)$ (autoregressivo de ordem 1), isto é

$$
X_{i}=a X_{i-1}+\epsilon_{i}, \quad a \in(-1,1),
$$

onde os $\epsilon_{i}$ 's formam um ruído Gaussiano com média 0 e variância $\sigma_{\epsilon}^{2}$.

Neste caso, é possível mostrar que para $n$ grande,

$$
\operatorname{Var}\left(\bar{X}_{n}\right) \sim \sigma^{2}\left(1+\frac{2 a}{1-a}\right) n^{-1}=\sigma^{2} c(a) n^{-1}
$$

e novamente a variância da média amostral tende a zero como função de $n$. O símbolo " " na equação (2.2) denota equivalência assintótica, isto é,

$$
f(n) \sim g(n), \text { quando } n \rightarrow \infty \Leftrightarrow \lim _{n \rightarrow \infty} \frac{f(n)}{g(n)}=1 .
$$

\footnotetext{
${ }^{1}$ Será comum ao longo do trabalho nos referirmos à um processo estocástico com memória longa simplesmente como processo com memória longa.
} 
Em geral, a variância da média amostral para muitos modelos de séries temporais (p.ex ARMA) e processos Markovianos é, para $n$ grande, dada por

$$
\operatorname{Var}\left(\bar{X}_{n}\right) \sim \sigma^{2} c(\rho) n^{-1}
$$

onde

$$
c(\rho)=1+\lim _{n \rightarrow \infty} n^{-1} \sum_{i \neq j} \rho(i, j),
$$

e $\rho(i, j)$ é a autocorrelação entre $X_{i}$ e $X_{j}$.

A variância (2.3) generaliza (2.1), mas tal generalização pode não ser suficiente. Existem modelos para os quais a variância de $\bar{X}_{n}$ difere de (2.1) não apenas através da constante $c(\rho)$, mas também pela velocidade com a qual esta tende a zero. Se houver algum indício de que a variância da média amostral tende a zero mais lentamente, é interessante modelar esse decaimento explicitamente. Assim, um modelo mais geral deveria admitir para $\kappa \in(0,1)$ e $n$ grande que

$$
\operatorname{Var}\left(\bar{X}_{n}\right) \sim \sigma^{2} c(\rho) n^{-\kappa}
$$

onde $c(\rho)$ é definido como

$$
c(\rho)=1+\lim _{n \rightarrow \infty} n^{\kappa-2} \sum_{i \neq j} \rho(i, j) .
$$

Se por simplicidade assumirmos que $\rho(i, j)=\rho(|i-j|)$, isto é, a correlação depende apenas da distância temporal entre $X_{i}$ e $X_{j}$, e possível mostrar, para $n$ grande, que

$$
\sum_{k=-(n-1)}^{n-1} \rho(k) \sim \text { constante } \cdot n^{1-\kappa},
$$

e como $\kappa<1$ segue que

$$
\sum_{k=-\infty}^{\infty} \rho(k)=\infty .
$$

Assim, as correlações decaem para zero tão lentamente de modo que elas não são somáveis, e este fenômeno recebe o nome de dependência de longo alcance ou memória longa, onde o parâmetro $\kappa$ mede a intensidade dessa memória. Observe que se $\kappa=1$ o processo ainda pode ter uma estrutura de dependência, mas esses processos são ditos possuir memória curta.

Grande parte das definições de dependência de longo alcance que aparecem na literatura são baseadas em propriedades de segunda ordem de um processo estocástico. Tais propriedades incluem o comportamento assintótico das covariâncias, densidade espectral e variância de somas parciais. A popularidade de tais definições são históricas e práticas: propriedades de segunda ordem são simples e fáceis de estimar dos dados.

Definição 2.1 (Processos estacionários de segunda ordem com memória longa). Considere um processo estacionário de segunda ordem $X=\left\{X_{t}, t \geq 0\right\}$ que assume valores reais, com média comum 0 e função de autocorrelação $\rho(\tau)$. O processo $X$ é dito um processo com memória longa (PML) se existir um número real $\kappa \in(0,1)$ tal que,

$$
\lim _{\tau \rightarrow \infty} \frac{\rho(\tau)}{c|\tau|^{-\kappa}}=1, \quad \text { para alguma constante } 0<c<\infty,
$$

ou simplesmente $\rho(\tau) \sim c|\tau|^{-\kappa}, \tau \rightarrow \infty$.

Note que o limite da definição acima implica na relação (2.4). Quando $\kappa=1 \mathrm{o}$ processo tem memória curta (PMC), como observado anteriormente, e as correlações são somáveis. Além disso, 
quando $\kappa>1$, temos que $\sum_{\tau} \rho(\tau)=0$ e o processo é dito antipersistente.

Conhecer as covariâncias (ou correlações e variância $\sigma^{2}$ ) equivale a conhecer a densidade espectral $f$ definida por:

$$
f(\lambda)=\frac{\sigma^{2}}{2 \pi} \sum_{k=-\infty}^{\infty} \rho(k) e^{i k \lambda} .
$$

Se para $|k|$ suficientemente grande temos que $\rho(k) \sim c|k|^{-\kappa} \operatorname{com} \kappa \in(0,1)$, então $f(\lambda) \sim c_{f}|\lambda|^{\kappa-1}$ quando $\lambda \rightarrow 0\left(0<c_{f}<\infty\right)$. Assim, a definição 2.1 implica que a densidade espectral de um processo estacionário com memória longa é infinita na frequência 0 , fenômeno este comumente mencionado como ruído $1 / f$ (Beran, 1994).

A definição acima serve apenas para processos estacionários de segunda ordem, razão pela qual faz sentido falar de objetos como variância, covariância e correlação do processo. No caso de processos com segundo momento infinito esses objetos não estão definidos e uma definição mais geral deve ser apresentada. Embora a definição acima não seja voltada à processos que não possuem segundo momento finito, é importante observar que qualquer série observada terá momentos finitos, de modo que a presença de memória longa pode ser detectada por muitos métodos baseados nessa definição. Heyde e Yang (1997) fornecem uma definição para memória longa mais geral que abrange estes casos.

Definição 2.2 (Processos não estacionários com memória longa). Considere um processo de segunda ordem $\left\{X_{t}, t \geq 1\right\}$ (não necessariamente estacionário) com média 0 e o processo "média em blocos"

$$
Y_{t}^{(m)}=\frac{\sum_{j=t m-m+1}^{t m} X_{j}}{\sum_{j=t m-m+1}^{t m} E\left(X_{j}^{2}\right)} .
$$

Dizemos que $\left\{X_{t}, t \geq 1\right\}$ é um processo com memória longa se

$$
\lim _{m \rightarrow \infty}\left(\sum_{j=t m-m+1}^{t m} E\left(X_{j}^{2}\right)\right) \operatorname{Var}\left(Y_{t}^{(m)}\right)=\infty .
$$

A Definição 2.2 acima implica na Definição 2.1 quando $\left\{X_{t}, t \geq 1\right\}$ é um processo estacionário de segunda ordem (Heyde e Yang, 1997).

Para lidar com casos onde a variância pode ser infinita, Heyde e Yang (1997) dizem que $\left\{X_{t}, t \geq\right.$ $1\}$ é um processo com memória longa se

$$
\frac{\left(\sum_{j=t m-m+1}^{t m} X_{j}\right)^{2}}{\sum_{j=t m-m+1}^{t m} X_{j}^{2}} \stackrel{P}{\rightarrow} \infty,
$$

e mostram que esta definição também implica na definição usual para $\left\{X_{t}, t \geq 1\right\}$ estacionário de segunda ordem.

Embora a deteç̧ão da presença de memória longa utilizando as condições acima seja complicada, Heyde e Yang (1997) apresentam o seguinte teorema:

Teorema 2.1. Seja $X=\left\{X_{t}, t \geq 0\right\}$ um processo estocástico com incrementos estacionários. Suponha que $X$ seja autossimilar com parâmetro de autossimilaridade $H$ e que $E\left(X_{1}\right)^{\alpha}<\infty$ para algum $0<\alpha<2$. Se $H>\frac{1}{\alpha}$, então $X$ é um processo com memória longa com parâmetro de memória $d=H-\frac{1}{\alpha}$.

Veremos na próxima seção o que significa um processo ser autossimilar com parâmetro de autossimilaridade $H$. Vale observar que o Teorema 2.1 fornece uma ferramenta simples para determinar se um processo estocástico autossimilar com segundo momento infinito possui memória longa. 


\subsection{Alguns processos com memória longa}

Ao longo das próximas seções apresentaremos alguns processos que possuem memória longa e que serão objeto de estudo deste trabalho do ponto de vista inferencial. Antes de apresentarmos estes processos, vamos definir o conceito de autossimilaridade, conceito este que viabilizou o grande avanço obtido por Mandelbroot e seu colaboradores no desenvolvimento de modelos com memória longa no final da década de 60 (Mandelbrot e Van Ness, 1968; Mandelbrot e Wallis, 1968, 1969a,b,c).

\subsubsection{Processos autossimilares}

O conceito de autossimilaridade foi teoricamente introduzido por Kolmogorov em 1941, mas foi somente no final da década de 60 que processos estocásticos com essas características foram desenvolvidos. Um exemplo simples de autossimilaridade vem da geometria, onde objetos com essa característica são conhecidos como fractais autossimilares. Intuitivamente, temos que uma forma geométrica é dita autossimilar se as mesmas estruturas geométricas são observadas independente da distância que se observa a mesma. A Figura 2.1 apresenta o chamado triângulo de Sierpenski, um exemplo de fractal autossimilar.

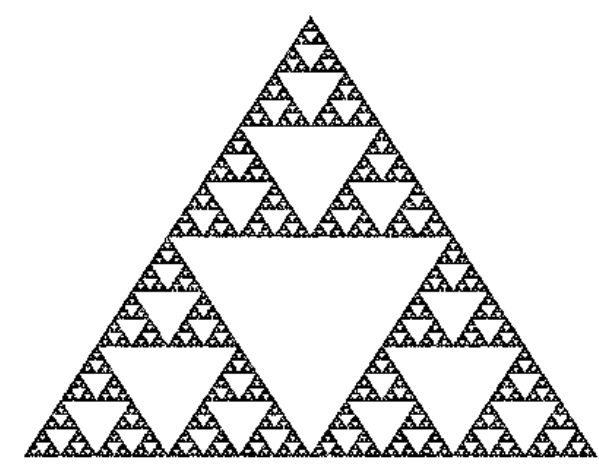

Figura 2.1: Triângulo de Sierpenski.

Em um contexto probabilístico, autossimilaridade é definida em termos da distribuição finito dimensional de um processo estocástico.

Definição 2.3 (Processos autossimilares). Um processo estocástico $\left\{Y_{t}, t \geq 0\right\}$ é dito autossimilar com parâmetro de autossimilaridade $H$ se, para qualquer constante $c>0$,

$$
\left\{Y_{c t}, t \geq 0\right\}=\left\{c^{H} Y_{t}, t \geq 0\right\},
$$

onde essa igualdade significa igualdade das distribuições finito dimensional.

Intuitivamente a definição anterior diz que trajetórias de um processo autossimilar são qualitativamente idênticas, independentemente da distância que se observa as mesmas. Isso não significa que a mesma figura se repete conforme nos aproximamos dela (como no exemplo geométrico apresentado na Figura 2.1), e sim que as propriedades probabilísticas permanecem inalteradas.

Suponha que $\left\{Y_{t}, t \geq 0\right\}$ seja um processo autossimilar com parâmetro de autossimilaridade $H$. Segue por definição que $Y_{t} \stackrel{D}{=} t^{H} Y_{1}$ para $t>0$, onde “吕” denota igualdade em distribuição. Quando $t \rightarrow \infty$ (respectivamente $t \rightarrow 0$ ) essa propriedade implica em:

1. $H<0 \Rightarrow Y_{t} \stackrel{D}{\rightarrow} 0$ (respectivamente $H<0$ e $\left.Y_{t} \neq 0 \Rightarrow\left|Y_{t}\right| \stackrel{D}{\rightarrow} \infty\right)^{2}$.

2. $H=0 \Rightarrow Y_{t} \stackrel{D}{=} Y_{1}$ (respectivamente $H=0 \Rightarrow Y_{t} \stackrel{D}{=} Y_{1}$ ).

\footnotetext{
${ }^{2} \mathrm{O}$ símbolo " $\stackrel{D}{\rightarrow}$ " denota convergência em distribuição
} 
3. $H>0$ e $Y_{t} \neq 0 \Rightarrow\left|Y_{t}\right| \stackrel{D}{\rightarrow} \infty$ (respectivamente $\left.H>0 \Rightarrow Y_{t} \stackrel{D}{\rightarrow} 0\right)$.

Excluindo o caso trivial $Y_{t} \equiv 0$, essas propriedades implicam que $\left\{Y_{t}, t \geq 0\right\}$ é estacionário somente quando $H=0$, mas tal caso corresponde ao processo constante igual a $Y_{1}$. Para modelar dados que pareçam estacionários devemos considerar processos autossimilares com incrementos estacionários. Além disso, devemos nos restringir aos valores $H>0$, pois se $H<0$ temos que $\left\{Y_{t}, t \geq 0\right\}$ não é um processo mensurável (Vervaat, 1985, 1987).

Suponha que $Y_{0}=0$ com probabilidade 1 e assuma, sem perda de generalidade que $\mathrm{E}\left(Y_{1}\right)=0$, o que implica pela autossimilaridade que $\mathrm{E}\left(Y_{t}\right)=0$ para todo $t$. Seja $s<t$ e denote por $\sigma^{2}=$ $\mathrm{E}\left(Y_{1}^{2}\right)=\mathrm{E}\left(Y_{t}-Y_{t-1}\right)^{2}$ a variância do processo de incrementos $\left\{X_{t}\right\}=\left\{Y_{t}-Y_{t-1}\right\}$, ou seja, estamos considerando nesse caso apenas processos com primeiro e segundo momento finitos. Segue que,

$$
\mathrm{E}\left[\left(Y_{t}-Y_{s}\right)^{2}\right]=\mathrm{E}\left[\left(Y_{t-s}-Y_{0}\right)^{2}\right]=\mathrm{E}\left(Y_{t-s}^{2}\right)=\mathrm{E}\left[(t-s)^{H} Y_{1}\right]^{2}=\sigma^{2}(t-s)^{2 H} .
$$

Por outro lado,

$$
\mathrm{E}\left[\left(Y_{t}-Y_{s}\right)^{2}\right]=\mathrm{E}\left(Y_{t}^{2}\right)+\mathrm{E}\left(Y_{s}^{2}\right)-2 \mathrm{E}\left(Y_{t} Y_{s}\right)=\sigma^{2} t^{2 H}+\sigma^{2} s^{2 H}-2 \operatorname{Cov}\left(Y_{t}, Y_{s}\right) .
$$

Combinando (2.5) e (2.6) segue que a função de covariância de um processo autossimilar $\left\{Y_{t}\right\}$ é dada por

$$
\gamma_{Y}(t, s)=\operatorname{Cov}\left(Y_{t}, Y_{s}\right)=\frac{\sigma^{2}}{2}\left[t^{2 H}+s^{2 H}-(t-s)^{2 H}\right]
$$

Isso implica, para todo $k$, que a covariância entre $X_{i}$ e $X_{i+k}(i=1,2,3, \ldots)$ do processo de incrementos é igual a

$$
\gamma(k)=\frac{\sigma^{2}}{2}\left[|k+1|^{2 H}+|k-1|^{2 H}-2|k|^{2 H}\right]
$$

de modo que a correlação correspondente é dada por

$$
\rho(k)=\frac{1}{2}\left[|k+1|^{2 H}+|k-1|^{2 H}-2|k|^{2 H}\right] .
$$

Suponha que $k>0$ e observe que $\rho(k)=\frac{1}{2} k^{2 H} g\left(k^{-1}\right)$ onde $g(x)=(1+x)^{2 H}+(1-x)^{2 H}-2$. Quando $k \rightarrow \infty$ temos que $g\left(k^{-1}\right) \rightarrow g(0)=0$. Para $0<H<1$ e $H \neq 1 / 2$, a expansão em série de Taylor de $g(x)$ na vizinhança de zero é $2 H(2 H-1) x^{2}$ e assim,

$$
\frac{\rho(k)}{H(2 H-1) k^{2 H-2}} \rightarrow 1, \text { quando } k \rightarrow \infty .
$$

Para $k$ geral, quando $|k|$ tende ao infinito, temos a equivalência assintótica $\rho(k) \sim H(2 H-$ 1) $|k|^{2 H-2}$. Assim, se $1 / 2<H<1$ concluímos, pela definição 2.1 , que o processo de incrementos de um processo autossimilar com parâmetro de autossimilaridade $H$ é um processo com memória longa e, nesse caso, a intensidade da memória é determinada pelo parâmetro de autossimilaridade $H$. Isso não ocorre quando o processo de incrementos de um processo autossimilar não possui segundo momento finito, como é o caso dos processos estáveis a serem introduzidos posteriormente.

Concluímos esta subseção voltando ao cálculo da variância da média amostral que serviu como motivação na seção 2.1. Se $X_{1}, \ldots, X_{n}$ é uma série do processo de incrementos de um processo autossimilar $\left\{Y_{t}\right\}$ com segundo momento finito e parâmetro de autossimilaridade $H$ então,

$$
\bar{X}_{n}=n^{-1} \sum_{i=1}^{n} X_{i}=n^{-1} Y_{n}=n^{-1} n^{H} Y_{1}=n^{H-1} Y_{1},
$$

e assim

$$
\operatorname{Var}\left(\bar{X}_{n}\right)=n^{2 H-2} \operatorname{Var}\left(Y_{1}\right)=\sigma^{2} n^{2 H-2}
$$


Se $H=1 / 2$ temos o resultado clássico (2.1). Se o processo $\left\{X_{t}\right\}$ for Gaussiano com média $\mu$ e variância $\sigma^{2}$ então $n^{1-H}\left(\bar{X}_{n}-\mu\right) / \sigma$ terá distribuição normal padrão e essa estatística pode ser usada para calcular intervalos de confiança para $\mu$. Se o objetivo for fazer previsões para observações futuras de uma série com memória longa, os intervalos de confiança obtidos da maneira tradicional, isto é, utilizando modelos com memória curta, irão produzir intervalos de previsão que subestimam as observações futuras. A Figura 2.2 ilustra essa falha dos modelos tradicionais em uma série de dados reais que apresenta memória longa.

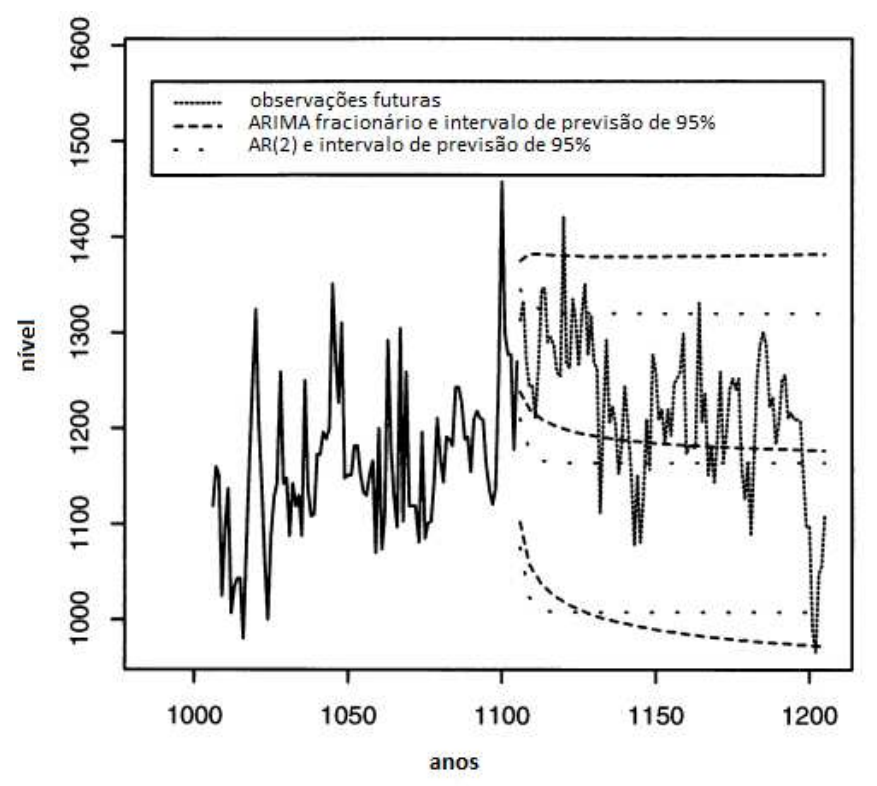

Figura 2.2: Niveis mínimos anuais do rio Nilo e intervalos de previsão obtidos utilizando-se dois modelos Gaussianos: Um com memória longa (ARIMA fracionário) e um com memória curta (autoregressivo de ordem 2).

\subsubsection{Processos de Hermite}

Os processos de Hermite compreendem uma importante classe de processos autossimilares com memória longa que inclui o movimento Browniano fracionário e o processo de Rosenblatt como casos particulares. Os incrementos estacionários desses dois processos são muito utilizados como modelos para dados que apresentam memória longa.

Definição 2.4 (Processos de Hermite). Seja $k \geq 1$ um número inteiro e $H \in\left(\frac{1}{2}, 1\right)$. Um processo de Hermite, denotado por $\left\{Z_{t}^{H, k}, t \in \mathbb{R}\right\}$, é um processo autossimilar com parâmetro de autossimilaridade $H$ que admite a seguinte representaçẫo em termos de integrais estocásticas de Wiener-Itô (veja Oksendal (2013)) calculada com respeito ao movimento Browniano $\left\{B_{y}, y \in \mathbb{R}\right\}$

$$
Z_{t}^{H, k}=c(H, k) \int_{\mathbb{R}^{k}}^{\prime}\left\{\int_{0}^{t} \prod_{j=1}^{k}\left(s-y_{j}\right)_{+}^{-\left(\frac{1}{2}+\frac{1-H}{k}\right)} d s\right\} d B_{y_{1}} \ldots d B_{y_{k}}
$$

onde $x_{+}=\max (x, 0) e$

$$
c(H, k)=\sqrt{\frac{H(2 H-1)}{k ! \beta\left(\frac{1}{2}-\frac{1-H}{k}, \frac{2-2 H}{k}\right)^{k}}}
$$

é uma constante normalizadora de modo que $\mathrm{E}\left(Z_{1}^{H, k}\right)^{2}=1$. A função $\beta(\cdot, \cdot)$ denota a função beta e o símbolo " $\int^{\prime}$ " indica que as diagonais onde $y_{i}=y_{j}(i \neq j)$ não devem ser consideradas na integração. O parâmetro $k$ indica a ordem do processo. 
Quando $k=1,\left\{Z_{t}^{H, 1}, t \in \mathbb{R}\right\}$ corresponde ao movimento Browniano fracionário ou fBm (fractional Brownian motion), o único processo Gaussiano nessa classe. O processo de incrementos estacionários obtido a partir do fBm é conhecido com ruído Gaussiano fracionário ou fGn (fractional Gaussian noise), sendo este amplamente utilizado como modelo para séries que exibem memória longa (Beran, 1994). Um fBm com $H=1 / 2$ se reduz ao movimento Browniano usual.

Quando $k=2$, o processo de Hermite correspondente é conhecido como processo de Rosenblatt, sendo o processo não Gaussiano mais simples nessa classe. Seu processo de incrementos estacionários é utilizado como modelo para séries estacionárias que exibem memória longa, mas que aparentam possuir distribuição marginal com assimetria positiva. O cálculo estocástico do processo de Rosenblatt é desenvolvido em Tudor (2008).

Os processos de Hermite de ordem $k \geq 2$ são importantes pois fornecem uma ampla classe de processos para modelar memória longa, possibilitando desvios significativos do fBm e outros processos Gaussianos sem a necessidade de recorrer à construções de processos a partir de complicadas equações diferenciais estocásticas baseadas no fBm.

Os processos de Hermite admitem outras representações que são detalhadas em Pipiras e Taqqu (2010). A representação mais utilizada, devido o seu papel fundamental no desenvolvimento do cálculo estocástico do processo, simulações e previsões, é a representação em um intervalo de tempo limitado. No caso $k=1$, que corresponde ao $\mathrm{fBm}$, temos que $\left\{Z_{t}^{H, 1}, t \in[0, T]\right\}$ é dado por

$$
B_{t}^{H}=: Z_{t}^{H, 1}=\int_{0}^{t} K^{H}(t, s) d B_{s}
$$

onde $\left\{B_{t}, t \in[0, T]\right\}$ é o movimento Browniano padrão (Tudor, 2008) e, para $t>s$

$$
K^{H}(t, s)=c(H, 1) \int_{s}^{t}\left(\frac{s}{u}\right)^{\frac{1}{2}-H}(u-s)^{H-\frac{3}{2}} d u .
$$

Segue de (2.9) que

$$
\frac{\partial K^{H}}{\partial t}(t, s)=c(H, 1)\left(\frac{s}{t}\right)^{\frac{1}{2}-H}(t-s)^{H-\frac{3}{2}} .
$$

Tudor (2008) demonstra que o processo de Rosenblatt $\left\{Z_{t}^{H, 2} t \in[0, T]\right\}$ pode ser representado por

$$
Z_{t}^{H, 2}=d(H) \int_{[0, t]^{2}}\left[\int_{y_{1} \vee y_{2}}^{t} \frac{\partial K^{H_{0}}}{\partial u}\left(u, y_{1}\right) \frac{\partial K^{H_{0}}}{\partial u}\left(u, y_{2}\right) d u\right] d B_{y_{1}} d B_{y_{2}},
$$

onde $H_{0}=(H+1) / 2$ e $d(H)=\sqrt{\left(2(2 H-1) / H(H+1)^{2}\right)}$ é uma constante de padronização. A representação acima é generalizada para $k \geq 3$ em Pipiras e Taqqu (2010), isto é

$$
Z_{t}^{H, k}=d(H, k) \int_{[0, t]^{k}}\left[\int_{y_{1} \vee \ldots \vee y_{k}}^{t} \prod_{j=1}^{k} \frac{\partial K^{H_{0}}}{\partial u}\left(u, y_{j}\right) d u\right] d B_{y_{1}} \ldots d B_{y_{j}}
$$

onde

$$
H_{0}=1-\frac{1-H}{k},
$$

e a constante de padronização é dada por

$$
d(H, k)=\sqrt{\frac{H(2 H-1)}{k !\left[H_{0}\left(2 H_{0}-1\right)\right]^{k}}} .
$$

Por ser autossimilar, o processo de incrementos estacionários de um processo de Hermite possui a estrutura de correlação dada por (2.8) para todo $k$ e, consequentemente, possui memória longa. 
Embora o fBm seja um caso particular dos processos de Hermite quando $H \in\left(\frac{1}{2}, 1\right)$, ele pode ser definido de outras formas (Beran, 1994) e por isso, o fBm admite valores de $H$ em $(0,1)$.

A Figura 2.3 apresenta trajetórias típicas (e seus respectivos incrementos) dos processo de Hermite de ordem 1 e 2 com parâmetro de memória $H=0.7$.
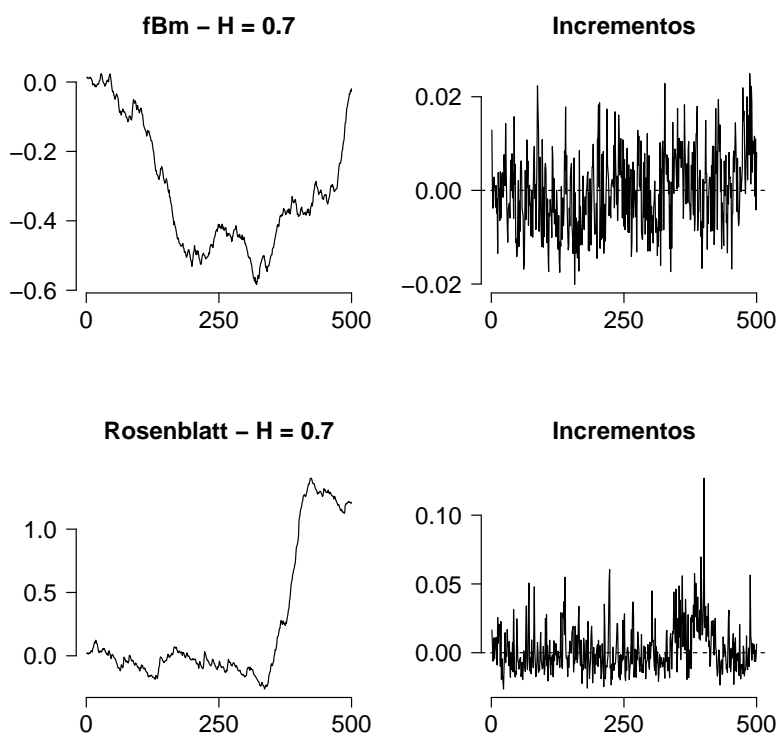

Figura 2.3: Trajetórias do processo de Hermite de ordem 1 (acima e a esquerda) e ordem 2 (abaixo e a esquerda) com parâmetro de memória $H=0.7$ e suas respectivas séries de incrementos (coluna direita).

As técnicas de simulação de processos de Hermite de ordem $k=1$ estão bem desenvolvidas. Um dos métodos mais difundidos para simular variáveis Gaussianas correlacionadas é conhecido como método de decomposição de Cholesky, cuja idéia parte do princípio de que uma matriz de covariância $\Sigma_{N \times N}$ é simétrica e positiva definida logo, pode ser decomposta como $\Sigma=A A^{T}$, onde $A_{N \times N}$ é definida como raiz quadrada de $\Sigma$. Se $\eta$ é um vetor ruído Gaussiano de comprimento $N$, então $Z=A \eta$ é um vetor aleatório Gaussiano com matriz de covariância $\Sigma$. De fato, note que

$$
\mathrm{E}\left(Z Z^{T}\right)=\mathrm{E}\left[(A \eta)(A \eta)^{T}\right]=A \mathrm{E}\left(\eta \eta^{T}\right) A^{T}=A A^{T}=\Sigma .
$$

Assim, o vetor aleatório $Z$ possui matriz de covariância $\Sigma$. Embora muito utilizado, este método é um dos menos eficientes para simular séries Gaussianas correlacionadas (em particular do fGn), pois o tempo computacional deste método cresce como a função $N^{3}$ (Bardet et al., 2003).

Outro método bastante utilizado é o algoritmo de Durbin-Levinson. Tal método explora o fato de que em séries Gaussianas estacionárias, as médias e variâncias condicionais podem ser obtidas recursivamente através de equações do tipo Yule-Walker. Os detalhes deste método podem ser obtidos em Brockwell e Davis (2013). Para simular uma série $X_{1}, \ldots, X_{N}$ com média zero e estrutura de covariância dada por (2.7), simule uma série i.i.d. $\left\{\eta_{i}, i \geq 1\right\}$ com distribuição comum $N(0,1)$. Faça $X_{1}=\gamma(0)^{1 / 2} \eta_{1}$ e obtenha $X_{2}, \ldots, X_{N}$ recursivamente através da relação

$$
X_{n+1}=\phi_{n, 1} X_{n}+\phi_{n, 2} X_{n-1}+\ldots+\phi_{n, n} X_{1}+v_{n}^{1 / 2} \eta_{n+1} .
$$

Os valores $v_{i}, i=1, \ldots, n$ e os coeficientes $\left\{\phi_{n, i}, i=1, \ldots, n\right\}$ são obtidos recursivamente da seguinte maneira: Faça $v_{0}=\gamma(0)$ e $v_{n}=v_{n-1}\left(1-\phi_{n, n}\right)^{2}$. Para $n \geq 1$,

$$
\begin{gathered}
\phi_{n, n}=\left[\gamma(n)-\sum_{j=1}^{n-1} \phi_{n-1, j} \gamma(n-j)\right] v_{n-1}^{-1}, \\
\phi_{n, i}=\phi_{n-1, i}-\phi_{n, n} \phi_{n-1, n-i}, \text { para } i<n .
\end{gathered}
$$


O tempo computacional deste método cresce como a função $N^{2}$.

Por último, seja $\left\{\xi_{i}, i \geq 1\right\}$ um sequência Gaussiana estacionária com média 0 e variância 1. Sottinen (2001) demonstra que

$$
B_{t}^{H, n}=\sum_{i=1}^{[n t]} n \int_{\frac{i-1}{n}}^{\frac{i}{n}} K^{H}\left(\frac{[n t]}{n}, s\right) d s \frac{\xi_{i}}{\sqrt{n}}, \quad t \in[0, T] . \quad n \geq 1,
$$

converge fracamente para o $\mathrm{fBm}\left\{B_{t}^{H}, t \in[0, T]\right\}$ padrão. ${ }^{3}$ Este último resultado viabiliza a simulação do fBm através de integração numérica.

Existem vários outros métodos para simular do proceso fBm ou fGn (Bardet et al. (2003) revisam uma dezena deles). Muitos estão implementados no software R-gui ${ }^{4}$, sendo que um dos mais populares e eficientes, proposto em Coeurjolly (2000), está implementado no pacote chamado dvfBm. Usuários de MATLAB podem obter gratuitamente códigos de simuladores do fGn na página do Professor Jean-Marc Bardet ${ }^{5}$.

Vamos apresentar agora um método de simulação de processos de Hermite de ordem $k \geq 2$. Tal método surgiu a partir de resultados obtidos em Taqqu (1978, 1979, 1975) e Dobrushin e Major (1979).

Definição 2.5 (Polinômios de Hermite). Um polinômio de Hermite de ordem $k$ é um polinômio da forma

$$
H_{k}(x)=(-1)^{k} e^{\frac{x^{2}}{2}} \frac{d^{k}}{d x^{k}} e^{-\frac{x^{2}}{2}} \quad(k=0,1,2, \ldots) .
$$

O seguinte teorema, demonstrado em Taqqu (1975), é a base para a simulação dos processos de Hermite de ordem $k \geq 2$.

Teorema 2.2 (Convergência fraca para um processo de Hermite). Seja $\mathfrak{D}[0,1]$ o espaço de todas as funções em $[0,1]$ cujas descontinuidades sejam no máximo de primeira ordem ${ }^{6}$. Suponha que $\left\{X_{i}, i \geq 1\right\}$ seja um fGn padrão com parâmetro de memória $H_{0}=1-(1-H) / k$ e $H_{k}(x)$ um polinômio de Hermite de ordem $k$. Então

$$
\frac{1}{d_{n}} \sum_{i=1}^{[n t]} H_{k}\left(X_{i}\right) \stackrel{n \rightarrow \infty}{\longrightarrow} Z_{t}^{H, k}, \text { fracamente em } \mathfrak{D}[0,1],
$$

onde, para $n \rightarrow \infty$,

$$
d_{n} \sim n^{H} \sqrt{\frac{k !\left[H_{0}\left(2 H_{0}-1\right)\right]^{k}}{H(2 H-1)}}
$$

é uma constante tal que $E\left(Z_{t}^{H, k}\right)^{2}=1$ (processo padrão).

Note que o teorema acima diz que os processos de Hermite de ordem $k \geq 2$ podem ser obtidos a partir de funções não lineares do fBm. A partir do teorema acima, uma trajetória aproximada $Z_{1}, \ldots, Z_{N}$ de $\left\{Z_{t}^{H, k}\right\}$ é obtida da seguinte maneira:

1. Simule uma trajetória de tamanho $m N$ do fGn padrão com parâmetro de memória $H_{0}=$ $1-(1-H) / k$.

2. Calcule

$$
Y_{j}=\frac{1}{(m N)^{H}} \sum_{i=1}^{m j} H_{k}\left(X_{i}\right), \text { para } j=1, \ldots, N,
$$

\footnotetext{
${ }^{3}[x]$ denota o maior inteiro menor que $x$.

${ }^{4}$ https://cran.r-project.org/

${ }^{5} \mathrm{http}: / /$ samm.univ-paris1.fr/Sofwares-Logiciels

${ }^{6}$ Uma função é descontínua de primeira ordem se ela der um salto em um determinado ponto. Os limites laterais neste ponto existem mas são distintos (incluindo $\pm \infty$ ).
} 
onde $H_{k}$ é o polinômio de Hermite de ordem $k$. Temos que $Y_{1}, \ldots, Y_{N}$ é uma trajetória de um processo de Hermite não padronizado de ordem $k$ nos tempos discretos $1 / N, 2 / N, \ldots, 1$.

3. Calcule

$$
Z_{j}=\frac{N^{H}}{d_{m N}} Y_{j} \text { para } j=1, \ldots, N
$$

onde $d_{m N}$ é a constante (2.10). A multiplicação por $N^{H}$ acima fornece, por autossimilaridade, uma trajetória de um processo de Hermite de ordem $k$ nos tempos discretos $1,2, \ldots, N$, enquanto a multiplicação por $d_{m N}$ faz com que a trajetória $Z_{1}, \ldots, Z_{N}$ tenha como processo adjacente o processo de Hermite padrão.

Note que nos passos acima estamos utilizando o Teorema 2.2 discretizando o tempo contínuo com $n=m N$ e $t=j / N(j=1, \ldots, N)$. O valor de $m$ controla a precisão dessa aproximação sendo que na prática $m$ é tomado entre 100 e 150 nas simulações com $k=2$ (Bardet e Tudor, 2010). Este método de simulação é pouco eficiente quando $N$ é grande, pois devemos simular uma série do fGn $m$ vezes maior.

Uma alternativa para simular de $\left\{Z_{t}^{H, 2}\right\}$ é apresentada em Torres e Tudor (2009) estendendo para $k=2$ o método de simulação do $\mathrm{fBm}$ através de integração numérica. Neste caso foi demonstrado que sendo

$$
F\left(t, y_{1}, y_{2}\right):=d(H, 2) \mathbb{I}_{[0, t]}\left(y_{1}\right) \mathbb{I}_{[0, t]}\left(y_{2}\right) \int_{y_{1} \vee y_{2}}^{t} \frac{\partial K^{H_{0}}}{\partial u}\left(u, y_{1}\right) \frac{\partial K^{H_{0}}}{\partial u}\left(u, y_{2}\right) d u
$$

então

$$
Z_{t}^{H, 2, n}=\sum_{\substack{i, j=1 \\ i \neq j}}^{[n t]} n^{2} \int_{\frac{i-1}{n}}^{\frac{i}{n}} \int_{\frac{j-1}{n}}^{\frac{j}{n}} F\left(\frac{[n t]}{n}, u, v\right) d v d u \frac{\xi_{i}}{\sqrt{n}} \frac{\xi_{j}}{\sqrt{n}}, \quad t \in[0, T], \quad n \geq 1,
$$

converge fracamente para o processo de Rosenblatt $\left\{Z_{t}^{H, 2}, t \in[0, T]\right\}$. Este resultado também pode ser utilizado para simular do processo de Rosenblatt $\left\{Z_{t}^{H, 2}, t \in[0, T]\right\}$ através de integração numérica. A generalização desse método para $k$ geral é apresentada em Sun e Cheng (2014).

Assim como o fBm, o processo de Rosenblatt pode ser simulado com o auxílio de wavelets (Abry e Pipiras, 2006). Códigos MATLAB para a simulação do processo de Rosenblatt podem ser obtidos gratuitamente na página do Professor Jean-Marc Bardet ${ }^{7}$.

\subsubsection{Movimento estável fracionário}

O movimento estável fracionário ou fSm (fractional stable motion) é um processo autossimilar que possui incrementos estacionários e podem exibir memória longa. Estes processos são considerados como uma extensão do movimento Browniano fracionário no sentido de admitir distribuições marginais com caudas pesadas (variância infinita). Essas características produzem o chamado efeito Noé (ou Noah effect), fazendo referência ao personagem bíblico e os efeitos climáticos extremos que este vivenciou.

Antes de definirmos tal processo, devemos definir o que é uma variável aleatória com distribuição estável. Essa classe foi descoberta por Paul Lévy em 1919 quando ainda se acreditava que as distribuições Gaussianas eram as únicas estáveis em contextos que envolviam o teorema central do limite (Kotz et al., 2006).

Definição 2.6 (Variáveis estáveis). Dizemos que uma v.a. X possui distribuição estável se existem parâmetros $\alpha \in(0,2],|\beta| \leq 1, \gamma>0$ e $\delta \in \mathbb{R}$, tais que a função característica de $X$ possui a seguinte forma

\footnotetext{
${ }^{7}$ http://samm.univ-paris1.fr/Sofwares-Logiciels
} 


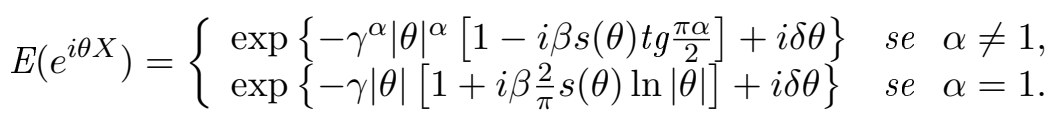

onde

$$
s(\theta)= \begin{cases}1 & \text { se } \theta>0 \\ 0 & \text { se } \theta=0 \\ -1 & \text { se } \theta<0\end{cases}
$$

O parâmetro $\alpha$ é dito indice de estabilidade ou de cauda e controla o peso da cauda da distribuição, $\beta$ controla a assimetria, $\gamma$ é um parâmetro de escala e $\delta$ um parâmetro de localização. Quando $\alpha<2$ dizemos que $X$ possui distribuição $\alpha$-estável.

A definição acima estabelece, de maneira equivalente (Samorodnitsky e Taqqu, 1994), que $X$ é uma v.a. $\alpha$-estável se ela possuir um domínio de atração, isto é, se existirem uma sequência i.i.d. $\left\{Y_{i}, i \geq 1\right\}$, uma sequência de números positivos $\left\{d_{n}, n \geq 1\right\}$ e uma sequência de números reais $\left\{a_{n}, n \geq 1\right\}$ tais que

$$
\frac{Y_{1}+\ldots Y_{n}}{d_{n}}+a_{n} \stackrel{D}{\rightarrow} X .
$$

Isto significa que as distribuições $\alpha$-estáveis são as únicas que podem ser obtidas como limite de somas padronizadas de variáveis i.i.d e são importantes pois generalizam o teorema central do limite. Vamos denotar uma variável aleatória com distribuição estável por $S(\alpha, \beta, \gamma, \delta)$. As Figuras 2.4 e 2.5 nos mostram as formas que essa distribuição assume variando os parâmetros $\alpha$ e $\beta$.
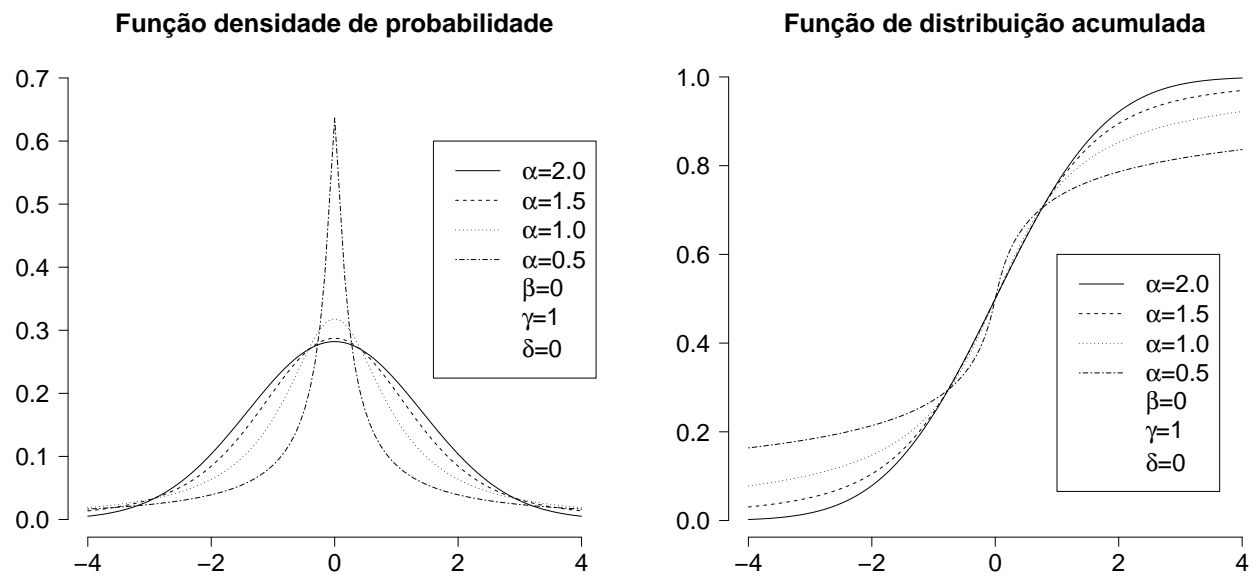

Figura 2.4: Funções densidade (esquerda) e distribuição acumulada (direita) da distribuição $\alpha$-estável com parâmetros $\beta=0, \gamma=1, \delta=0$ e $\alpha$ variando em $\{2.0,1.5,1.0,0.5\}$.

Apenas algumas v.a.'s com distribuição estável admitem função densidade e função de distribuição em forma analítica. Em geral, essas funções são obtidas numericamente. Casos particulares incluem $^{8}$ :

1. $S(2,0, \sigma / \sqrt{2}, \mu) \stackrel{D}{=} N\left(\mu, \sigma^{2}\right)$,

2. $S(1,0,1,0) \stackrel{D}{=} \operatorname{Cauchy}(0,1)$,

3. $S(1 / 2,1, \gamma, \delta)$ é dita distribuição de Lévy (Samorodnitsky e Taqqu, 1994).

A simulação de amostras de uma distribuição estável é algo bem estabelecido e pode ser feito em praticamente todos os softwares estatísticos. No caso do R-gui o pacote stabledist também

\footnotetext{
${ }^{8} \mathrm{O}$ símbolo $\stackrel{D}{=}$ denota igualdade em distribuição.
} 
Função densidade de probabilidade

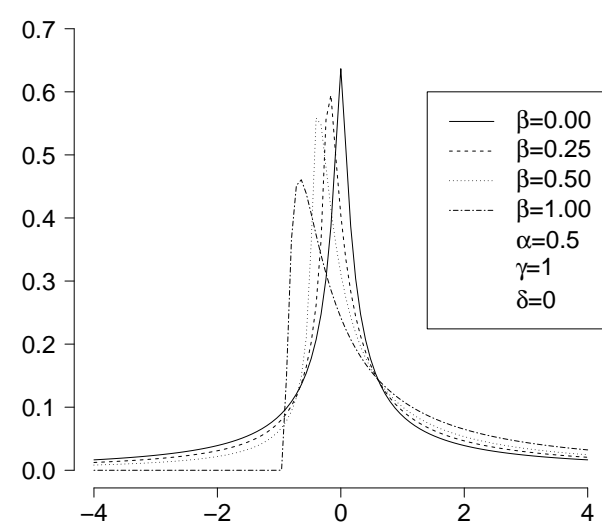

Função de distribuição acumulada

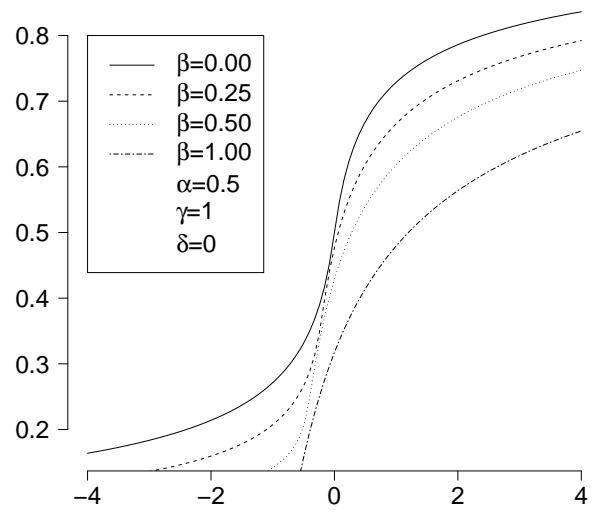

Figura 2.5: Funções densidade (esquerda) e distribuição acumulada (direita) da distribuição $\alpha$-estável com parâmetros $\alpha=0.5, \gamma=1, \delta=0$ e $\beta$ variando em $\{0.00,0.25,0.50,1.00\}$.

calcula numericamente as funções de densidade e de distribuição. Maiores detalhes sobre simulação de distribuições $\alpha$-estáveis podem ser obtidas em Chambers et al. $(1976,1987)$.

Definição 2.7 (Movimento de Lévy Simétrico). O movimento de Lévy simétrico, movimento estável ou voo de Lévy é um processo estocástico que possui incrementos independentes e estacionários com distribuição marginal $S(\alpha, 0, \gamma, 0)$.

O movimento de Lévy é a contraparte do movimento Browniano quando $\alpha<2$, pois enquanto o movimento Browniano é obtido pelas somas acumuladas de v.a.'s normais independentes, o movimento de Lévy é obtido pela soma acumulada de v.a's $\alpha$-estáveis simétricas e independentes. Seu processo de incrementos, conhecido como ruído estável, possui parâmetro de autossimilaridade $H=1 / \alpha$. Observe que $H$ é apenas o parâmetro de autossimilaridade, já que os incrementos do processo não possuem memória.

Definição 2.8 (Movimento estável fracionário). Seja $H \in(0,1)$ e $\alpha \in(0,2]$. O movimento estável fracionário (fSm), denotado por $\left\{L_{t}^{H, \alpha}, t \in \mathbb{R}\right\}$ é um processo estocástico que admite a seguinte representação integral

$$
L_{t}^{H, \alpha}=\int_{-\infty}^{0}\left(|t-s|^{H-\frac{1}{\alpha}}-|s|^{H-\frac{1}{\alpha}}\right) d L_{s}^{\alpha}+\int_{0}^{t}|t-s|^{H-\frac{1}{\alpha}} d L_{s}^{\alpha},
$$

onde $\left\{L_{t}^{\alpha}, t \in \mathbb{R}\right\}$ é o movimento de Lévy simétrico com parâmetro de estabilidade $\alpha$.

O fSm é uma extensão do fBm para o caso $\alpha$-estável. De fato, o fBm é um fSm com $\alpha=2$, pois o fBm admite representação integral como na Definição 2.8 mas neste caso, a integral deve ser calculada com respeito ao movimento Browniano (Samorodnitsky e Taqqu, 1994). Assim como o fBm, este processo é autossimilar com parâmetro de autossimilaridade $H$ e possui incrementos estacionários, que chamaremos de ruído estável fracionário ou fSn (fractional stable noise). Quando $H=1 / \alpha$, o fSm se reduz ao movimento de Lévy, enquanto o fSn se reduz a um ruído estável. Quando $H>1 / \alpha$ o fSn é um PML estacionário de acordo com o Teorema 2.1.

A Figura 2.6 compara trajetórias do fBm com $H=0.8$ e $\alpha=2$ e do fSm com mesmo valor de $H$ e $\alpha=1.25$, enquanto a Figura 2.7 compara suas respectivas séries de incrementos.

Um método de simulação de trajetórias de um fSn pode ser feita através de uma aproximação de sua representação integral. Samorodnitsky e Taqqu (1994) propõem o seguinte método: Considere o kernel dado por

$$
K^{H, \alpha}= \begin{cases}x^{H-\frac{1}{\alpha}}-(x-1)^{H-\frac{1}{\alpha}}, & x>1, \\ x^{H-\frac{1}{\alpha}}, & 0<x \leq 1,\end{cases}
$$


$\mathrm{fBm}: \mathrm{H}=0.8(\alpha=2)$

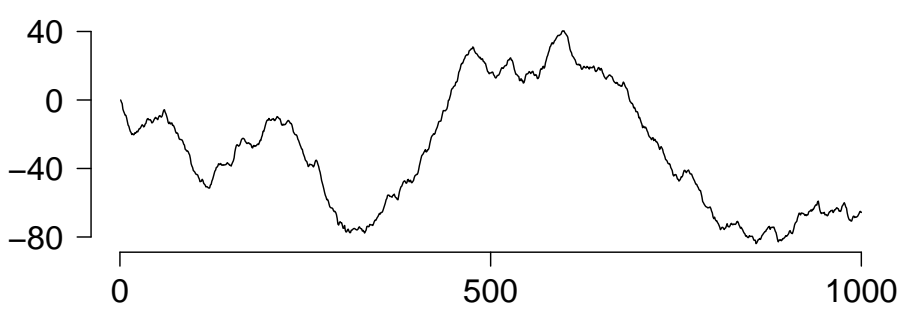

fSm: $H=0.8(\alpha=1.25)$

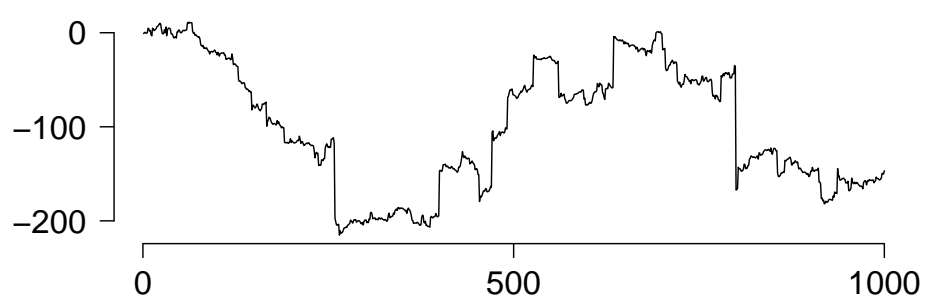

Figura 2.6: Trajetória amostral do fBm (acima) e do fSm (abaixo) com parâmetro de autossimilaridade $H=0.8$.

$$
f G n: H=0.8(\alpha=2)
$$

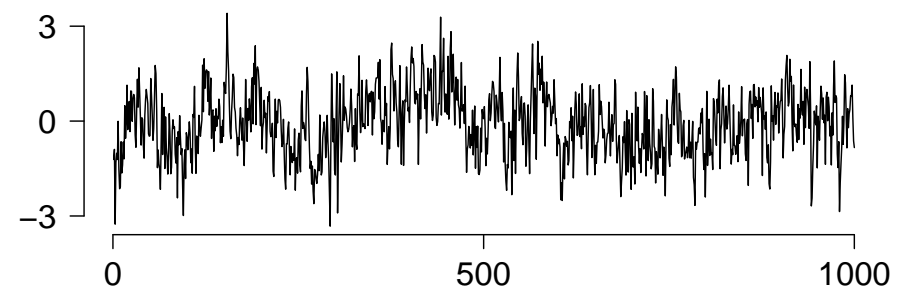

$\mathrm{fSn}: \mathrm{H}=0.8(\alpha=1.25)$

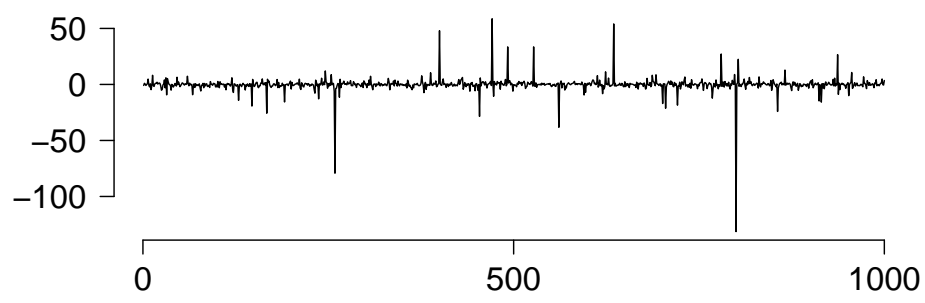

Figura 2.7: Trajetória amostral do $f G n$ (acima) e do fSn (abaixo) com parâmetro de autossimilaridade $H=0.8$.

e calcule 


$$
Y_{j}=\sum_{u=1}^{M m} K^{H, \alpha}\left(\frac{u}{m}\right) \xi_{j-(u / m)}, \quad j=1, \ldots, T,
$$

onde as $\xi$ 's são v.a.'s i.i.d. $\alpha$-estáveis simétricas com $\alpha \in(0,2]$. $M$ e $m$ são números inteiros, onde $M$ determina o truncamento nos limites de integração, $m$ define a discretização da integral e $T \leq M$ é o comprimento da série que se deseja simular. Os valores $Y_{1}, \ldots, Y_{T}$ formam uma trajetória do fSn. Para aproximar uma trajetória do fSm basta calcular $T^{-H} \sum_{j=1}^{[t T]} Y_{j}, 1 / T \leq t \leq 1$.

Um método mais preciso e eficiente, que utiliza transformada rápida de Fourier, é apresentado em Stoev e Taqqu (2004). O código MATLAB deste método pode ser obtido gratuitamente na página de um dos autores ${ }^{9}$.

\subsubsection{Processo gama modulado generalizado}

O processo gama-modulado generalizado ou GGMp (generalized gamma-modulated process) foi proposto em Andrade et al. (2015) como uma generalização, em termos do parâmetro de memória, do processo gama-modulado desenvolvido em Iglesias et al. (2011), onde os autores abordaram um problema da área de finanças supondo que o parâmetro de memória era fixo e conhecido.

No caso do GGMp o parâmetro de memória também é desconhecido e essa generalização torna a estrutura de dependência do processo mais flexível. Tal processo é definido em termos do movimento Browniano padrão e do processo gama a ser definido a seguir.

Definição 2.9 (Processo gama). Um processo gama $\left\{\gamma_{t}(a, b), t>0\right\}$ é um processo cuja distribuição marginal no tempo t é gama com média at/b e variância at $/ b^{2}$.

Dado um processo gamma $\left\{\gamma_{t}(a, b), t>0\right\}$, segue da definição acima que:

(i) $k \gamma_{t}(a, b) \stackrel{D}{=} \gamma_{t}(a, b / k)$;

(ii) $\mathrm{E}\left[\gamma_{t}(a, b)^{n}\right]=b^{-n} \Gamma(a t+n) / \Gamma(a t)$ para $n \geq 0$;

(iii) $\mathrm{E}\left[e^{\theta \gamma_{t}(a, b)}\right]=(1-\theta / b)^{-a t}$, para $\theta<b$;

(iv) Sua função característica $\phi_{\gamma_{t}(a, b)}$ é dada por

$$
\phi_{\gamma_{t}(a, b)}(u)=\left(1-\frac{u}{b} i\right)^{-a t} .
$$

(v) $\operatorname{Corr}\left[\gamma_{s}(a, b), \gamma_{t}(a, b)\right]=\sqrt{s / t}$, para $s<t$.

(vi) Dado $k>0$, a densidade $f_{k}(y)$ do incremento $\gamma_{t+k}(a, b)-\gamma_{t}(a, b)$ é dada por

$$
f_{k}(y)=\frac{b^{a k}}{\Gamma(a k)} y^{a k-1} e^{-b y}, \quad y>0 .
$$

Assim como em Andrade et al. (2015), vamos considerar $a=b=1 / 2$ e $\gamma_{t}(1 / 2,1 / 2):=\gamma_{t}$.

Definição 2.10 (Processo gama-modulado generalizado). Dado um número real $\beta \in[-1,1]$, o processo gamma-modulado generalizado $\left\{{ }^{\beta} X_{t}, t>0\right\}$ é definido por

$$
{ }^{\beta} X_{t}=B_{t} \gamma_{t}^{\beta}, \quad \text { para } t>0,
$$

onde $\left\{B_{t}, t>0\right\}$ é o movimento Browniano padrão e $\left\{\gamma_{t}, t>0\right\}$ é um processo gamma tal que $\gamma_{t}=\gamma_{t}(1 / 2,1 / 2)$.

\footnotetext{
${ }^{9}$ http://dept.stat.lsa.umich.edu/ ${ }^{\text {sstoev/ }}$
} 
Em particular, temos que $\left\{{ }^{0} X_{t}, t>0\right\}$ corresponde ao movimento Browniano usual, enquanto $\left\{{ }^{-0.5} X_{t}, t>0\right\}$ corresponde ao processo $t$-Student com memória longa abordado em Iglesias et al. (2011). Este último é uma generalização do processo $t$-Sudent apresentado em Heyde e Leonenko (2005). Claramente temos que $\mathrm{E}\left({ }^{\beta} X_{t}\right)=0$. A Figura 2.8 apresenta realizações típicas do GGMp para diferentes valores de $\beta$.
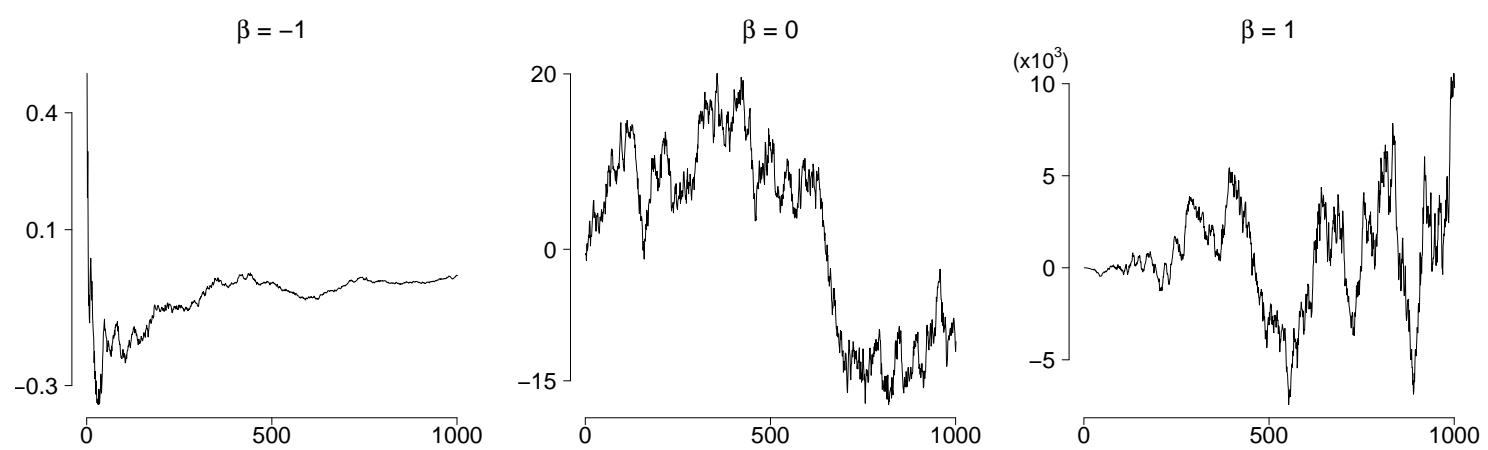

Figura 2.8: Trajetórias amostrais do processo gama-modulado generalizado (GGMp) para diferentes valores de $\beta$.

Tipicamente, trajetórias geradas pelo GGMp exibem heterocedasticidade e quando $\beta \in[-1,0)$, o processo apresenta alta volatilidade em seu início. Essa característica foi demonstrada e chamada de "explosão em zero" em Andrade et al. (2015). Além disso, quando o tempo cresce, a variabilidade decresce de acordo com o parâmetro de memória.

Alternativamente, quando $\beta \in(0,1]$, o processo diverge conforme o tempo cresce a uma taxa controlada novamente pelo parâmetro de memória.

Considere agora o processo de incrementos do GGMp dado por $\left\{\Delta\left({ }^{\beta} X_{t}\right)=B_{t} \gamma_{t}^{\beta}-B_{t-1} \gamma_{t-1}^{\beta}, t>\right.$ $0\}$ (a Figura 2.9 apresenta algumas trajetórias para diferentes valores de $\beta$ ). Por simplicidade vamos chamar este processo de GGMn (generalized gamma-modulated noise). Neste caso $\operatorname{Var}\left[\Delta\left({ }^{\beta} X_{t}\right)\right] \sim$ $t^{2 \beta}$ para $\beta \in[-1,1]$, o que implica que $\log \operatorname{Var}\left[\Delta\left({ }^{\beta} X_{t}\right)\right] \sim 2 \beta \log t$ (veja Andrade et al. (2015)). Logo, para $t$ suficientemente grande, a variância do processo de incrementos é linear com respeito a $t$ em escala logarítmica e esta relação linear é controlada pelo valor $2 \beta$. Este resultado pode ser facilmente visualizado empiricamente através de simulações do processo de incrementos como mostra a Figura 2.10, onde $\operatorname{Var}\left[\Delta\left({ }^{\beta} X_{t}\right)\right]$ é aproximada pela variância amostral nos tempos $1, \ldots, 100$ a partir de 1000 trajetórias simuladas de tamanho 100 . Note que o resultado assintótico pode ser visualizado a partir de $\log t \approx 3$, ou seja, para $t \approx 20$ para todos os valores de $\beta$.
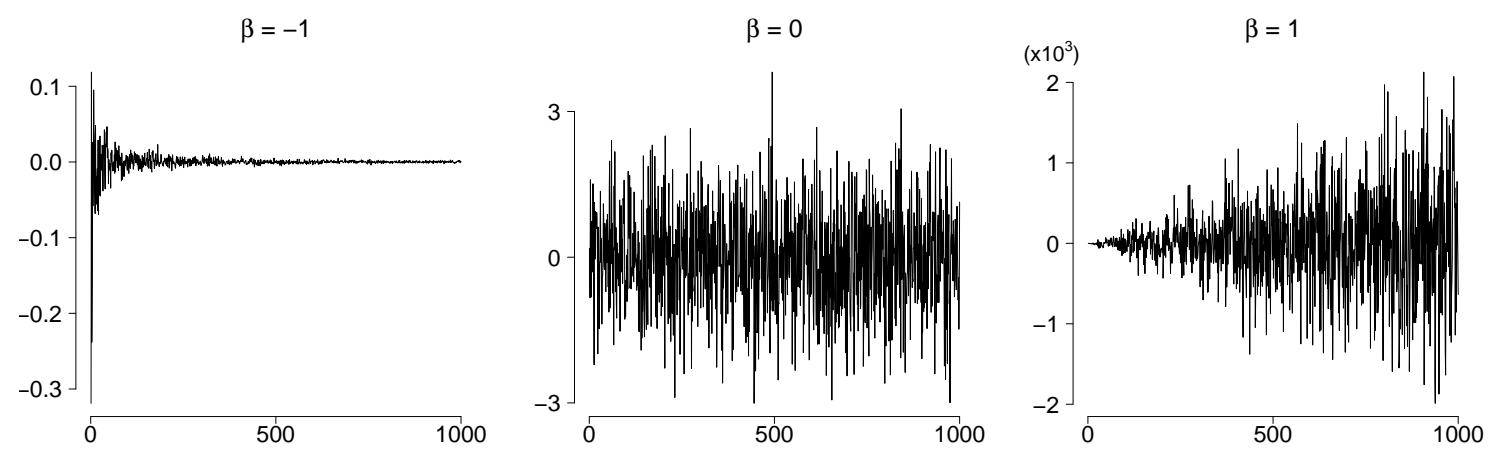

Figura 2.9: Trajetórias amostrais do processo de incrementos do processo gama-modulado generalizado (GGMn) para diferentes valores de $\beta$. 


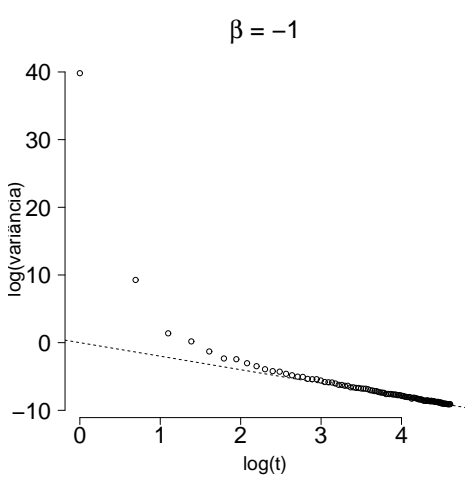

$\beta=0.5$

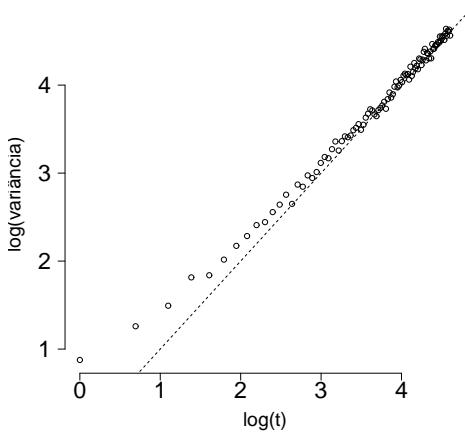

$\beta=-0.5$

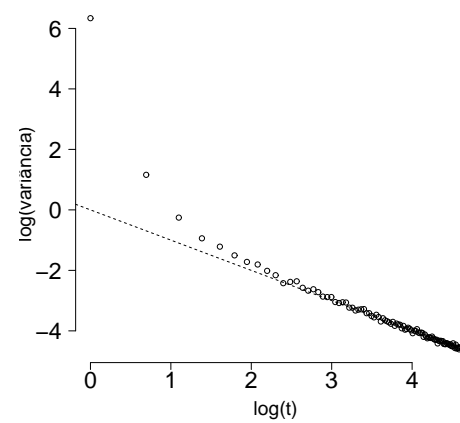

$\beta=0.7$

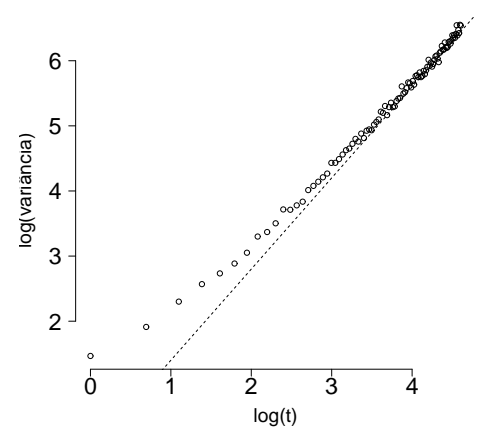

$\beta=0$

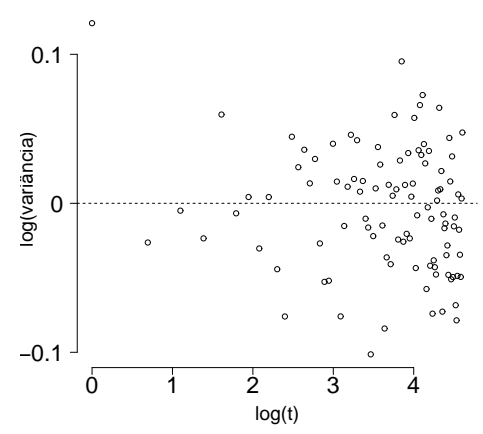

$\beta=1$

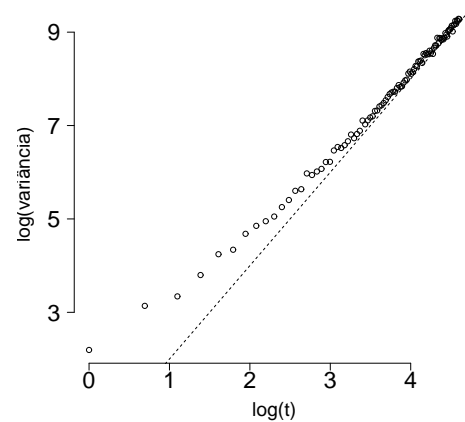

Figura 2.10: Distribuição amostral de $\operatorname{Var}\left[\Delta\left({ }^{\beta} X_{t}\right)\right]$ para $t=1,2, \ldots, 100$ obtida através de 1000 trajetórias simuladas do GGMn para diferentes valores de $\beta$.

O seguinte resultado diz respeito à estrutura de dependência do processo.

Proposição 2.1. Seja $\gamma(k)=\operatorname{Cov}\left[\Delta\left({ }^{\beta} X_{i}\right), \Delta\left({ }^{\beta} X_{i+k}\right)\right](1,2, \ldots)$ a função de autocovariância de lag $k$ do GGMn. Temos que

$$
\lim _{k \rightarrow \infty} \frac{\gamma(k)}{k^{\beta-1}}=1 \text { para } \beta \in(-1,0) \cup(0,1),
$$

e $\gamma(k)=0$ para todo $k$ quando $\beta=0$.

Demonstração. Vamos considerar $k \geq 3$, devido a explosão em zero quando $\beta \in(-1,0)$. Logo

$$
\begin{aligned}
\gamma(k)= & \mathrm{E}\left(\Delta\left({ }^{\beta} X_{3}\right) \Delta\left({ }^{\beta} X_{k+3}\right)\right) \\
= & \mathrm{E}\left(\left(B_{3} \gamma_{3}^{\beta}-B_{2} \gamma_{2}^{\beta}\right)\left(B_{k+3} \gamma_{k+3}^{\beta}-B_{k+2} \gamma_{k+2}^{\beta}\right)\right) \\
= & \mathrm{E}\left(B_{3} \gamma_{3}^{\beta} B_{k+3} \gamma_{k+3}^{\beta}\right)-\mathrm{E}\left(B_{3} \gamma_{3}^{\beta} B_{k+2} \gamma_{k+2}^{\beta}\right) \\
& -\mathrm{E}\left(B_{2} \gamma_{2}^{\beta} B_{k+3} \gamma_{k+3}^{\beta}\right)+\mathrm{E}\left(B_{2} \gamma_{2}^{\beta} B_{k+2} \gamma_{k+2}^{\beta}\right) \\
= & \mathrm{E}\left(B_{3} B_{k+3}\right) \mathrm{E}\left(\gamma_{3}^{\beta} \gamma_{k+3}^{\beta}\right)-\mathrm{E}\left(B_{3} B_{k+2}\right) \mathrm{E}\left(\gamma_{3}^{\beta} \gamma_{k+2}^{\beta}\right) \\
& -\mathrm{E}\left(B_{2} B_{k+3}\right) \mathrm{E}\left(\gamma_{2}^{\beta} \gamma_{k+3}^{\beta}\right)+\mathrm{E}\left(B_{2} B_{k+2}\right) \mathrm{E}\left(\gamma_{2}^{\beta} \gamma_{k+2}^{\beta}\right) \\
= & 3 \mathrm{E}\left(\gamma_{3}^{\beta} \gamma_{k+3}^{\beta}-\gamma_{3}^{\beta} \gamma_{k+2}^{\beta}\right)-2 \mathrm{E}\left(\gamma_{2}^{\beta} \gamma_{k+3}^{\beta}-\gamma_{2}^{\beta} \gamma_{k+2}^{\beta}\right) .
\end{aligned}
$$


Note que

$$
\begin{aligned}
\mathrm{E}\left(\gamma_{t}^{\beta} \gamma_{s}^{\beta}\right) & =\frac{1}{2^{t / 2}} \frac{1}{\Gamma((t-s) / 2) \Gamma(s / 2)} \int_{0}^{\infty} \int_{0}^{\gamma_{t}} \gamma_{t}^{\beta} \gamma_{s}^{\beta+s / 2-1}\left(\gamma_{t}-\gamma_{s}\right)^{(t-s) / 2-1} e^{-\gamma_{t} / 2} d \gamma_{s} d \gamma_{t} \\
& =\frac{1}{2^{t / 2}} \frac{1}{\Gamma\left(\frac{t-s}{2}\right) \Gamma(s / 2)} \int_{0}^{\infty} \int_{0}^{\gamma_{t}} \gamma_{s}^{\beta}\left(\gamma_{t}-\gamma_{s}\right)^{(t-s) / 2-1} \gamma_{s}^{s / 2-1} d \gamma_{s} \gamma_{t}^{\beta} e^{-\gamma_{t} / 2} d \gamma_{t} \\
& =\frac{1}{2^{t / 2}} \frac{1}{\Gamma\left(\frac{t-s}{2}\right) \Gamma(s / 2)} \int_{0}^{\infty} \int_{0}^{1} z^{\beta+s / 2-1}(1-z)^{(t-s) / 2-1} d z \gamma_{t}^{2 \beta+t / 2-1} e^{-\gamma_{t} / 2} d \gamma_{t} \\
& =\frac{1}{2^{t / 2}} \frac{1}{\Gamma\left(\frac{t-s}{2}\right) \Gamma(s / 2)} \frac{\Gamma(\beta+s / 2) \Gamma((t-s) / 2)}{\Gamma(\beta+t / 2)} \int_{0}^{\infty} \gamma_{t}^{2 \beta+t / 2-1} e^{-\gamma_{t} / 2} d \gamma_{t} \\
& =\frac{1}{2^{t / 2}} \frac{1}{\Gamma\left(\frac{t-s}{2}\right) \Gamma(s / 2)} \frac{\Gamma(\beta+s / 2) \Gamma((t-s) / 2)}{\Gamma(\beta+t / 2)} \frac{\Gamma(t / 2 \beta)}{(1 / 2)^{t / 2+2 \beta}} \\
& =4^{\beta} \frac{\Gamma(\beta+s / 2) \Gamma(2 \beta+t / 2)}{\Gamma(s / 2) \Gamma(\beta+t / 2)}
\end{aligned}
$$

Observe agora que $\Gamma(\beta+s / 2) / \Gamma(s / 2)=\Gamma(x+c) / \Gamma(x+1)$, onde $x=s / 2-1$ e $c=\beta+1$. Logo, para $\beta \in(-1,0)$, segue da inequação de Gautschi $^{10}$ (Kershaw, 1983) que

$$
\frac{\Gamma(\beta+s / 2)}{\Gamma(s / 2)}<\left(\frac{s}{2}-1\right)^{\beta} .
$$

Analogamente, temos que

$$
\frac{\Gamma(2 \beta+t / 2)}{\Gamma(\beta+t / 2)}<\left(\frac{t}{2}+\beta-1\right)^{\beta}
$$

Obtemos assim a equivalência assintótica $\mathrm{E}\left(\gamma_{t}^{\beta} \gamma_{s}^{\beta}\right) \sim(s t)^{\beta}$ para $\beta \in(-1,0)$. O mesmo resultado é obtido para o caso $\beta \in(0,1)$ bastando observar que $\Gamma(x+a) \rightarrow x^{a} \Gamma(x)$ para $a>0$ e $x$ suficientemente grande (Magnus et al., 2013). Claramente temos que $\mathrm{E}\left(\gamma_{t}^{\beta} \gamma_{s}^{\beta}\right)=1$ para $\beta=0$ e portanto,

$$
\left.\gamma(k)=\mathrm{E}\left(\gamma_{1}^{\beta} \gamma_{k+1}^{\beta}\right)-\mathrm{E}\left(\gamma_{1}^{\beta} \gamma_{k}^{\beta}\right)\right) \sim k^{\beta-1}, \quad \text { para } \beta \in(-1,0) \cup(0,1),
$$

e $\gamma(k)=0$ para $\beta=0$.

A simulação do GGMp é imediata, bastando observar que este processo é o produto da soma acumulada de variáveis $N(0,1)$ independentes com a soma acumulada de variáveis qui-quadrado com 1 grau de liberdade independentes e elevado a $\beta$. 


\section{Capítulo 3}

\section{Estimadores clássicos para o índice de memória}

Processos Gaussianos com memória longa foram amplamente estudados ao longo dos últimos 50 anos e vários métodos de estimação foram propostos. No entanto, nem sempre podemos assumir que os dados observados possuem natureza Gaussiana, o que torna complexa ou mesmo impossível a estimação do índice de memória. Mesmo sob a conveniente suposição de normalidade e estacionariedade, alguns problemas podem surgir, mesmo para processos simples como o fGn (apresentado na seção 2.2). Neste caso, embora a distribuição finito dimensional do processo seja dada pela distribuição normal multivariada, a matriz de covariância pode tornar-se computacionalmente singular quando o índice de memória aproxima-se de 1, fazendo com que métodos numéricos para obtenção do estimador de máxima verossimilhança falhem. Outro exemplo é que grande parte desses processos possuem apenas representações dadas por integrais estocásticas, dificultando o emprego de métodos inferencias que dependem da forma analítica de funções de densidade ou distribuição. Esses e outros problemas fazem com que a maioria das técnicas clássicas de estimação do índice de memória sejam aproximativas.

Em geral três abordagens são utilizadas para estimar o índice de memória em processos com memória longa:

- Técnicas gráficas ou heurísticas: Tais técnicas assumem que o processo gerador da série estudada é autossimilar ou assintoticamente autossimilar (Doukhan et al., 2003). Esse tipo de abordagem, em geral, é muito pobre em termos de viés e variabilidade (Taqqu et al., 1995), e são recomendadas apenas como uma ferramenta de inspeção visual para detectar a presença de memória longa nos dados. A técnica mais conhecida, que será apresentada posteriormente, é a análise R/S proposta em Hurst (1951).

- Modelos paramétricos: O parâmetro de memória é um dos vários parâmetros que determinam o modelo. Nesse contexto destacam-se os trabalhos de Dahlhaus (1989) e Fox e Taqqu (1986) que lidam com séries Gaussianas, enquanto Giraitis e Surgailis (1990) provam resultados similares para processos lineares. Giraitis e Taqqu (1999) propõem um método inferencial paramétrico para funções não lineares de processos Gaussianos. A abordagem paramétrica têm como principal desvantagem as restrições impostas pelo modelo como, por exemplo, assumir conhecida a verossimilhança ou conhecer a forma analítica da função de densidade espectral em todas as frequências.

- Modelos semiparamétricos: Trata-se do conjunto de técnicas mais utilizadas por serem mais flexíveis e robustas com respeito à especificação do modelo. Nesse caso, a memória longa é descrita por um parâmetro, mas a estrutura de dependência em curto alcance não é especificada ou é parcialmente especificada (Doukhan et al., 2003). As abordagens mais comuns nesse contexto exploram o comportamento da função de densidade espectral próximo da origem como observado na seção 2.1 . 
Vamos revisar alguns desses estimadores, que serão utilizados para comparar com o método proposto neste trabalho.

\subsection{Análise do variograma}

Vimos no final da seção 2.2.1 que se $X=\left\{X_{t}, t \in \mathbb{Z}\right\}$ é um processo autossimilar estacionário com parâmetro de autossimilaridade $H$ e $\mathbf{x}=\left(x_{1}, \ldots, x_{n}\right)$ uma trajetória amostral de $X$, então

$$
\lim _{n \rightarrow \infty} \frac{\operatorname{Var}\left(\bar{x}_{n}\right)}{C_{\text {var }} n^{2 H-2}}=1,
$$

onde $\bar{x}_{n}$ denota a média amostral de $\mathbf{x}$ e $C_{v a r}$ é uma constante positiva.

O limite (3.1) continua válido para qualquer processo linear com memória longa (Beran et al., 2013). Consequentemente, para $n$ suficientemente grande, $\log \operatorname{Var}\left(\bar{x}_{n}\right) \sim \log C_{v a r}+(2 H-2) \log n$. Com base nessa equivalência assintótica, o variograma é construído da seguinte maneira:

1. Divida $\mathbf{x}$ em $m$ blocos disjuntos e adjacentes de tamanho $k$, de modo que $n=[m k]$, onde $[z]$ denota a parte inteira de $z$.

2. Calcule a média amostral $\bar{x}_{k}(j)(j=1, \ldots, m)$ de cada bloco.

3. Calcule a variância amostral

$$
s^{2}(k)=\frac{1}{m-1} \sum_{j=1}^{m}\left(\bar{x}_{k}(j)-\bar{x}\right)^{2}
$$

onde $\bar{x}=1 / m \sum_{j=1}^{m} \bar{x}_{k}(j)$.

4. Repita os passos $1-3$ para $k=2, \ldots, n / 2$. O variograma é o gráfico dos pontos $\left(\log k, \log s^{2}(k)\right)$.

Heuristicamente, quando $k$ cresce, $\operatorname{Var}\left(\bar{x}_{k}(j)\right) \sim C k^{2 H-2}$ para cada $j=1, \ldots, m$. Assim, $s^{2}(k)$ cresce aproximadamente a uma taxa $k^{2 H-2}$. Se a série observada for uma realização de um processo linear com memória longa e índice de memória $H$, os pontos $\left(\log k, \log s^{2}(k)\right)$ do variograma vão se concentrar em torno de uma reta com inclinação aproximadamente igual a $2 H-2$. Deste modo, uma estimativa $\hat{H}_{v a r}$ de $H$ é obtida por mínimos quadrados.

A Figura 3.1 apresenta o variogama de duas series simuladas; uma do fGn com $H=0.5$ (sequência de v.a.'s normais independentes) e outra do fGn com $H=0.8$ (v.a.'s fortemente dependentes). Note que quando o processo possui memória longa os pontos se concentram acima da linha com inclinação -1 que corresponde aos processos com memória curta.

\subsection{Análise $\mathrm{R} / \mathrm{S}$}

Dada uma trajetória amostral $\mathbf{x}=\left(x_{1}, \ldots, x_{n}\right)$ do processo estacionário de segunda ordem $X=\left\{X_{t}, t \in \mathbb{Z}\right\}$, a análise $\mathrm{R} / \mathrm{S}$ baseia-se no estudo do comportamento assintótico da seguinte estatística estudada por Hurst (1951)

$$
R / S(n)=\frac{1}{\hat{s}_{n}}\left[\max _{1 \leq k \leq n} \sum_{i=1}^{k}\left(x_{i}-\bar{x}_{n}\right)-\min _{1 \leq k \leq n} \sum_{i=1}^{k}\left(x_{i}-\bar{x}_{n}\right)\right],
$$

onde $\bar{x}_{n}$ e $\hat{s}_{n}^{2}$ denotam respectivamente a média e a variância amostral da trajetória x. Para processos autossimilares de parâmetro $H$ vale que

$$
\lim _{n \rightarrow \infty} \frac{R / S(n)}{C_{R / S} n^{H}}=1
$$



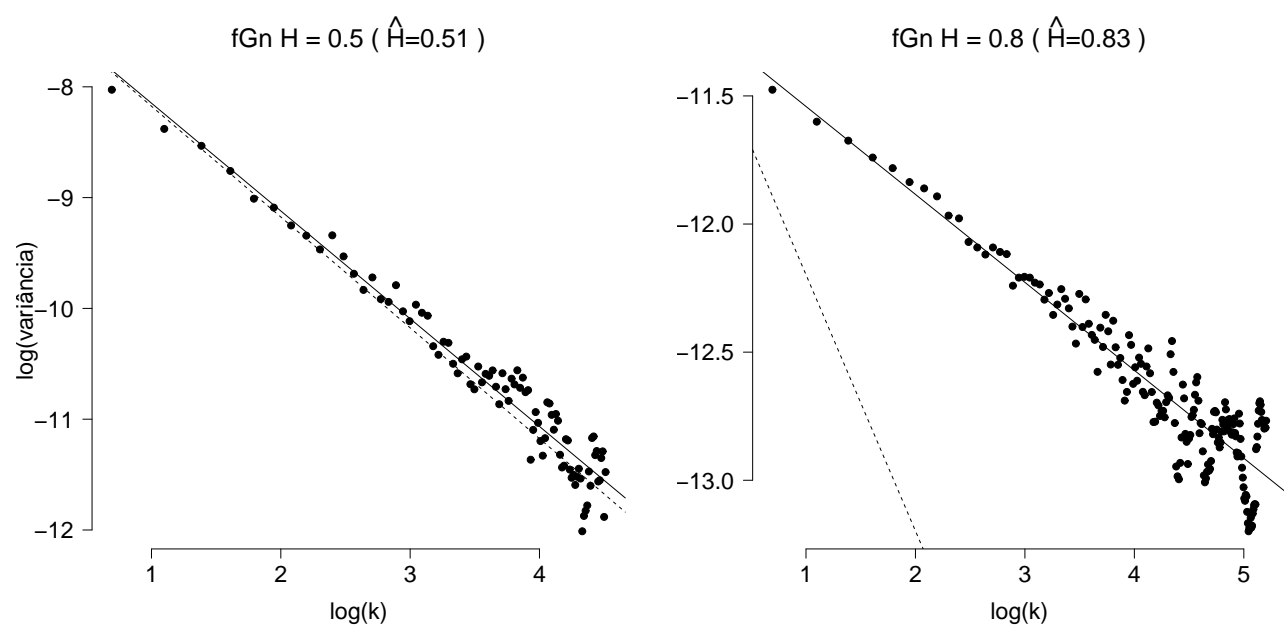

Figura 3.1: Variograma para séries simuladas do fGn com $H=0.5$ (esquerda) e $H=0.8$ (direita). A linha cheia foi obtida por minimos quadrados, enquanto a linha tracejada corresponde a inclinação -1 (memória curta).

onde $C_{R / S}$ é uma constante positiva (Beran et al., 2013).

Assim, temos que para $n$ suficientemente grande, $\log R / S(n) \sim H \log n+\log C_{R / S}$, e uma estimativa $\hat{H}_{R / S}$ de $H$ pode ser obtida de forma análoga ao método do variograma, isto é através da regressão de $R / S(n)$ em $n$ em escala logaritmica. O gráfico dos pontos $(\log (n), \log R / S(n))$ é conhecido como poxplot.

A Figura 3.2 apresenta os poxplots para séries simuladas do fGn com memória curta $(H=0.5)$ e com memória longa $(H=0.8)$. Neste caso, quando o processo possui memória longa os pontos se concentram acima da reta com inclinação 0.5.
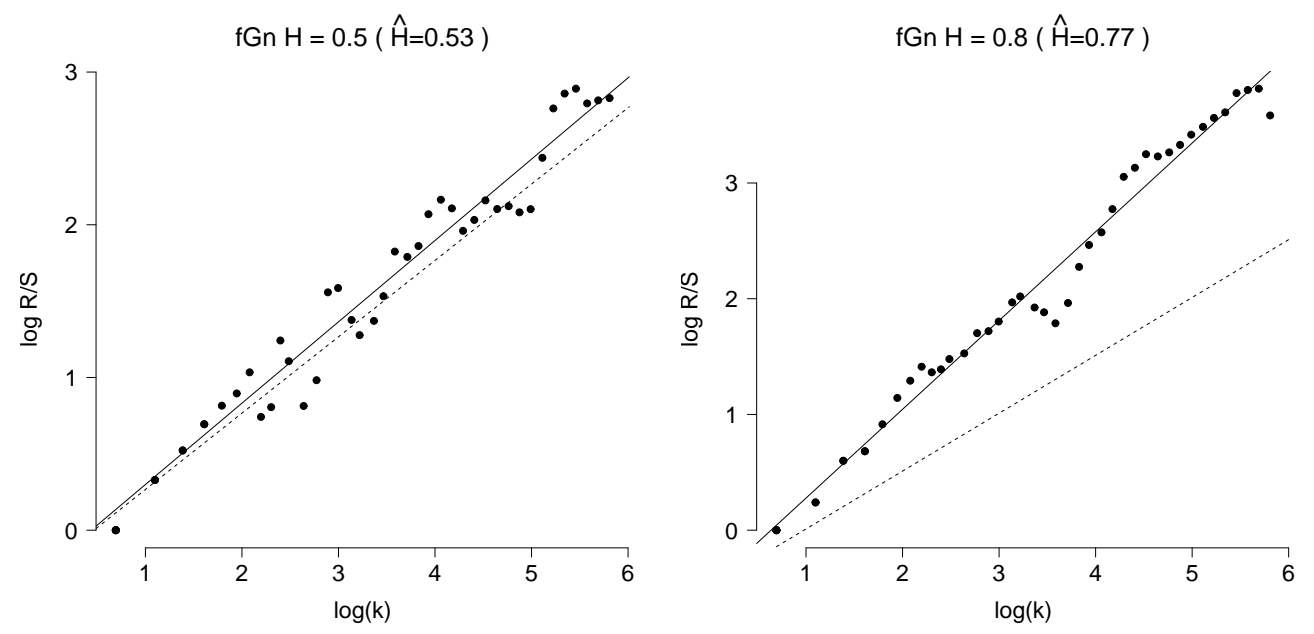

Figura 3.2: Poxplot para séries simuladas do fGn com $H=0.5$ (esquerda) e $H=0.8$ (direita). A linha cheia foi obtida por mínimos quadrados, enquanto a linha tracejada corresponde a inclinação 0.5 (memória curta).

\subsection{Análise local e global do periodograma}

O periodograma de um processo estacionário $X=\left\{X_{t}, t \in \mathbb{Z}\right\}$, é dado por 


$$
I_{n}(\lambda)=\frac{1}{2 \pi n}\left|\sum_{j=1}^{n} X_{j} e^{-i j \lambda}\right|^{2},
$$

onde $e^{i \cdot}$ é a função exponencial complexa. O periodograma é um estimador da densidade espectral de um processo (Hamilton, 1994).

Quando o processo $X_{t}$ possui variância finita e índice de memória longa $H$, seu periodograma é proporcional a $|\lambda|^{1-2 H}$ próximo da origem (Beran, 1994), isto é

$$
\lim _{|\lambda| \rightarrow 0} \frac{I_{n}(\lambda)}{C_{p e r}|\lambda|^{1-2 H}}=1
$$

onde $C_{\text {per }}$ é uma constante positiva.

Assim, a regressão de $I_{n}(\lambda)$ em $|\lambda|$ em escala logaritmica deve ter um coeficiente linear igual a $1-2 H$ para valores de $\lambda$ próximos de 0 , fornecendo uma aproximação $\hat{H}_{\text {per }}$ para o índice de memória $H$. Na prática, o periodograma é calculado nas frequências de Fourier, a saber $\lambda_{j}=2 \pi j / n$, onde $j=1, \ldots,[(n-1) / 2]$ e $n$ é o comprimento da série observada.

A Figura 3.3 apresenta a análise gráfica do periodograma para séries simuladas do fGn com memória curta $(H=0.5)$ e com memória longa $(H=0.8)$. Neste caso, quando o processo possui memória curta a reta obtida por mínimos quadrados tende a ter inclinação nula, passando a ter inclinação cada vez mais negativa conforme o índice de memória aumenta.
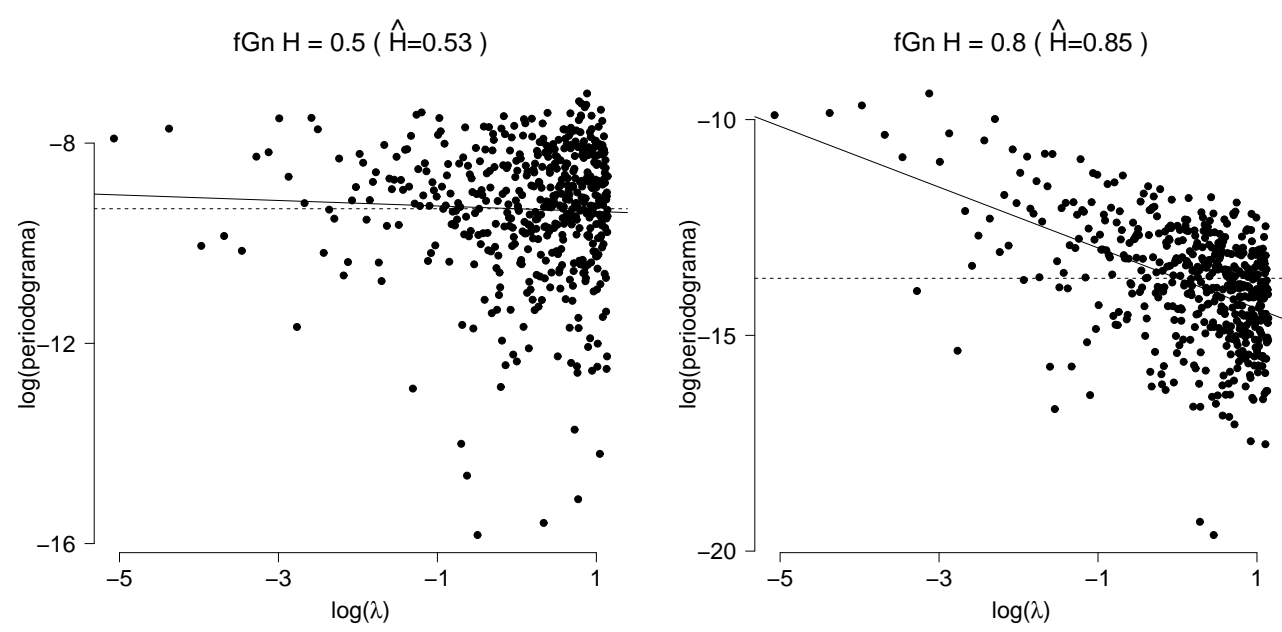

Figura 3.3: Periodograma para séries simuladas do $f G n$ com $H=0.5$ (esquerda) e $H=0.8$ (direita). A linha cheia foi obtida por mínimos quadrados, enquanto a linha tracejada corresponde a inclinação 0 (memória curta).

Seja $g$ a densidade espectral do processo $X$, Moulines e Soulier (1999) assumem que $g$ satisfaz a seguinte relação:

$$
g(\lambda)=g^{*}(\lambda)|1-\exp (i \lambda)|^{1-2 H},
$$

com $H \in\left(\frac{1}{2}, 1\right)$. A função $g^{*}$, contínua e não nula na origem, governa o comportamento de memória curta do processo. Note que $g$ caracteriza um processo com memória longa pois $g(\lambda) \sim g^{*}(0)|\lambda|^{1-2 H}$ quando $\lambda \rightarrow 0$.

Além disso, os autores também consideram a seguinte expansão de $\log g^{*}$ nas frequências de Fourier:

$$
\log g^{*}\left(\lambda_{j}\right)=\frac{1}{\sqrt{\pi}} \sum_{k=0}^{\infty} \theta_{k} \cos \left(k \lambda_{j}\right)
$$


O método do periodograma global ou FEXP (frequency-exponential), consiste em fixar uma ordem de aproximação $m$ para a série (3.5) e calcular a regressão linear do logaritmo do periodograma tendo como variáveis explicativas $\log \left|1-\exp \left(i \lambda_{j}\right)\right|$ e $\cos \left(k \lambda_{j}\right) / \sqrt{\pi}$, obtendo assim o estimador $\hat{H}_{F E X P}$ de $H$ (também é possível obter $\hat{\theta}=\left(\hat{\theta}_{1}, \ldots, \hat{\theta}_{m}\right)$, correspondente à memória curta do processo, com as devidas modificações).

\subsection{Análise DF}

A análise DF, ou simplesmente DFA (detrended fluctuation analysis) foi introduzida em Peng et al. (1994) para testar a presença de memória longa em sequências de DNA. Seja $\mathbf{x}=\left(x_{1}, \ldots, x_{n}\right)$ uma trajetória amostral de um processo estacionário de segunda ordem $X=\left\{X_{t}, t \in \mathbb{Z}\right\}$. A análise DF é feita de seguinte maneira:

1. Divida $\mathbf{x}$ em $m$ blocos disjuntos e adjacentes de tamanho $k$, de modo que $n=[m k]$.

2. Para cada bloco, faça a regressão de $T_{l}=\sum_{i=1}^{l} x_{i}$ em $l$ e estime a variância dos resíduos por

$$
s_{k}^{2}(j)=\frac{1}{m} \sum_{l=(j-1) k+1}^{j k}\left(T_{l}-\hat{\beta}_{0, j}-\hat{\beta}_{1, j}\right)^{2} \quad(j=1, \ldots, m),
$$

onde $\hat{\beta}_{0, j}$ e $\hat{\beta}_{1, j}$ são as estimativas de mínimos quadrados obtidas a partir do $j$-ésimo bloco.

3. Calcule

$$
F(k)=\frac{1}{m} \sum_{j=1}^{m} s_{k}^{2}(j)
$$

4. Repita os passos 1-3 para $k=3, \ldots, n / 2$ e obtenha o gráfico dos pontos $(\log k, \log F(k))$.

Heuristicamente, quando $k$ cresce, $F(k)$ cresce a uma taxa $k^{2 H}$. Logo, uma estimativa $\hat{H}_{D F A}$ de $H$ é obtida por mínimos quadrados de forma análoga ao método do variograma.

A Figura 3.4 apresenta os gráficos obtidos pela DFA para séries simuladas do fGn com memória curta $(H=0.5)$ e com memória longa $(H=0.8)$. Note que quando o processo possui memória longa os pontos se concentram acima da reta com inclinação 1 que corresponde aos processos com memória curta.
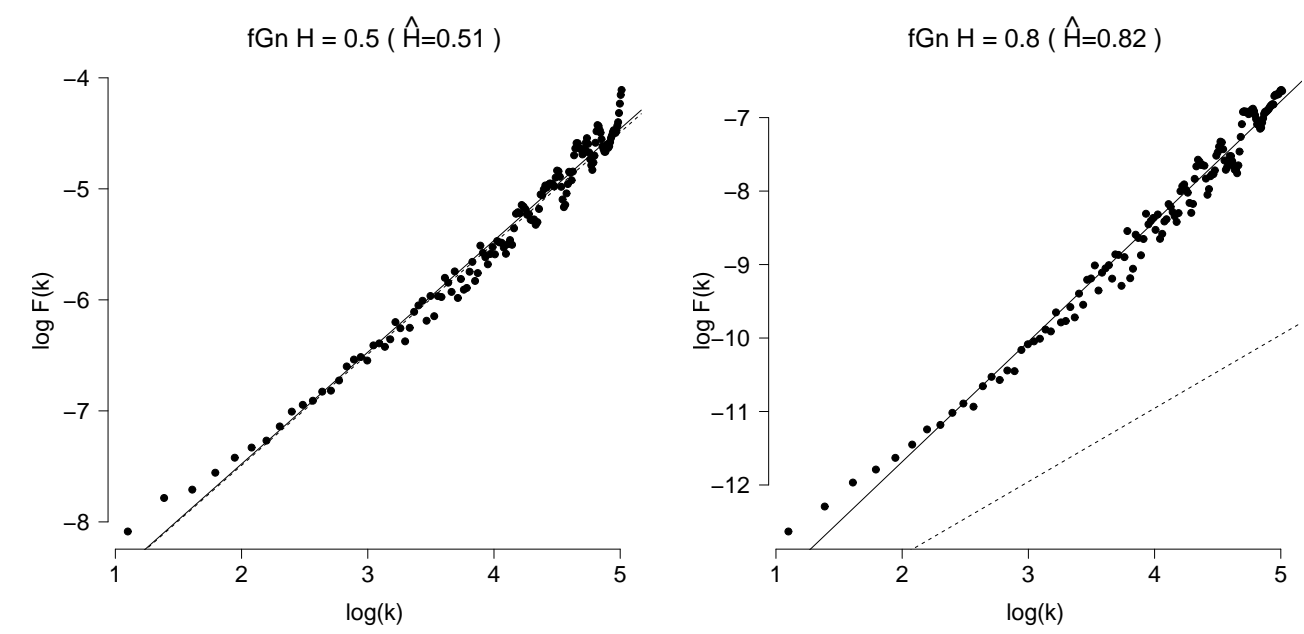

Figura 3.4: Gráficos obtidos pela DFA para séries simuladas do fGn com $H=0.5$ (esquerda) e $H=$ 0.8 (direita). A linha cheia foi obtida por mínimos quadrados, enquanto a linha tracejada corresponde a inclinação 1 (memória curta). 
Este método é similar ao método do variograma ou R/S, porém, este é menos sensível a tendência presente nos dados.

\subsection{Métodos baseados no estimador de Whittle}

Como observado no início desta seção, existem casos onde métodos numéricos para a obtenção do estimador de máxima verossimilhança falham devido a problemas relacionados com a natureza do processo como, por exemplo, matrizes de covariância que não são teoricamente singulares serem consideradas singulares do ponto de vista computacional, já que a precisão do computador é limitada (um dado número pode não ser nulo, mas ser tão pequeno que o computador o reconhece como tal). Além disso, a dimensão dessa matriz de covariância cresce com o comprimento da série observada, tornando métodos de máxima verossimilhança baseados na distribuição finito dimensional do processo pouco eficientes. O estimador de máxima verossimilhança também pode ser obtido através de métodos que lidam com a densidade espectral do processo, mas nem sempre a forma paramétrica dessa densidade é conhecida, ou esta é conhecida apenas parcilmente em algumas frequências. $\mathrm{O}$ método de Whittle (Whittle, 1953) foi criado como o objetido de aproximar a verossimilhança da série observada de processos estacionários. Este método envolve a minimização da seguinte função:

$$
U_{n}(\theta)=\int_{-\pi}^{\pi}\left[\log g(\lambda, \theta)+\frac{I_{n}(\lambda)}{g(\lambda, \theta)}\right] d \lambda .
$$

Podemos substituir $g$ por $h$ tal que $h=\beta g$ e $\int_{-\pi}^{\pi} \log h(\lambda, \theta) d \lambda=0$. O primeiro termo de (3.7) permanece inalterado exceto por uma divisão por $\beta$, que é tratado como um parâmetro extra, mas que não necessita ser estimado, uma vez que este não depende de $\theta$ e consequentemente não afeta a minimização de $U_{n}$ em $\theta$. Na prática, a integral em (3.7) é substituída por uma soma calculada nas primeiras $[(n-1) / 2]$ frequências de Fourier. O parâmetro $\theta$ será um vetor contendo o parâmetro de memória $H$ e parâmetros relacionados com a memória curta do processo como, por exemplo, parâmetros autoregressivos e de médias móveis.

Quando o processo estudado é Gaussiano, o estimador de Whittle $\hat{H}_{W}$ de $H$ converge para seu verdadeiro valor a uma taxa de $n^{1 / 2}$ e, neste caso, $n^{1 / 2}\left(\hat{H}_{W}-H\right)$ é assintoticamente normal (Fox e Taqqu, 1986). No caso dos incrementos do processo de Rosenblatt (que não é Gaussiano), $\hat{H}_{W}$ continua convergindo para seu verdadeiro valor, porém a uma taxa de $n^{1-H}$ e, neste caso, $n^{1-H}\left(\hat{H}_{W}-H\right)$ possui distribuição assintótica dada em termos da distribuição de Rosenblatt, como demonstrado recentemente em Bardet e Tudor (2014).

Um estudo empírico de Taqqu et al. (1995) mostra que o estimador de Whittle é o melhor estimador dentre vários estimadores (incluindo os apresentados até agora) quando utilizados em séries originadas por processos Gaussianos estacionários com memória longa.

O método de Whittle assume que a forma da densidade espectral do processo estudado seja conhecida em todas as frequências, o que pode não ser razoável na prática. Por este motivo, este método é dito global. Como vimos anteriormente, processos estacionários com memória longa apresentam um comportamento característico de suas densidades espectral para frequências próximas de zero. Este comportamento é explorado com o objetivo de flexibilizar o emprego do método de Whittle em processos mais gerais.

O estimador local de Whittle é um estimador semiparamétrico que especifica a forma da densidade espectral somente próximo da origem. O cálculo desse estimador envolve a inclusão de um valor $m<n / 2$ que deve satisfazer, quando $n \rightarrow \infty, 1 / m+m / n \rightarrow 0$. Para a densidade espectral de um processo estacionário com memória longa, temos que a versão discreta de (3.7) é dada por:

$$
U_{n}(c, H)=\frac{1}{m} \sum_{j=1}^{m}\left(\frac{I_{n}\left(\lambda_{j}\right)}{c \lambda_{j}^{1-2 H}}+\log c \lambda_{j}^{1-2 H}\right) .
$$

De onde obtemos que $\hat{c}=\frac{1}{m} \sum_{j=1}^{m} \frac{I_{n}\left(\lambda_{j}\right)}{\lambda_{j}^{1-2 H}}$ e assim, o valor $\hat{H}_{R}$ que minimiza $R_{n}(H)=U_{n}(\hat{c}, H)$ é 
dito estimador local de Whittle. Tal estimador converge em probabilidade para seu valor verdadeiro $H$ quando $n \rightarrow \infty$ e, quando o processo é Gaussiano, $m^{1 / 2}\left(\hat{H}_{R}-H\right)$ é assintoticamente normal, como demonstrado em Robinson (1995).

Neste trabalho iremos considerar o estimador FELW (fully extended local Whittle) introduzido em Abadir et al. (2007). Tal técnica baseia-se no estimador local de Whittle generalizando a definição do periodograma e da transformada rápida de Fourier, tornando possível a aplicação dessas duas funções em processos mais gerais. Além disso, a consistência desse estimador ainda é obtida em muitos casos onde as suposições de normalidade ou linearidade não são razoáveis.

O estimador de Whittle está implementado no software R-gui através do pacote longmemo baseando-se em Beran (1994). Usuários de S-PLUS podem obter implementações do estimador local e global de Whittle gratuitamente na página do professor Murad S. Taqqu${ }^{1}$. A implementação do estimador FELW (e dos demais) na linguagem MATLAB está disponível gratuitamente na página do Professor Jean-Marc Bardet ${ }^{2}$.

\subsection{Método de ondaletas}

O emprego de ondaletas (ou wavelets) para estimar o parâmetro de autossimilaridade e/ou de memória foi proposto pela primeira vez em Abry et al. (1998). Em seguida, Bardet et al. (2000) demonstrou a consistência desse estimador num contexto semiparamétrico Gaussiano. Por último, Roueff e Taqqu (2009) estabeleceram resultados similares em um contexto semiparamétrico para processos lineares. A partir daí, várias melhorias tem sido propostas nos mais diversos contextos. Apresentaremos a seguir uma idéia resumida do método de ondaletas.

Definição 3.1 (Ondaletas). Uma ondaleta é uma função $\psi: \mathbb{R} \rightarrow \mathbb{R}$ que deve satisfazer

$$
\begin{aligned}
& \text { a) } \int_{\mathbb{R}} \psi(t) d t=0, \\
& \text { b) } \psi \in L^{1}(\mathbb{R}) \cap L^{2}(\mathbb{R}), \quad \text { isto é, } \quad \int_{\mathbb{R}}|\psi(t)|^{k} d t=0, \quad(k=1,2) .
\end{aligned}
$$

Em geral as ondaletas consideradas são limitadas, centradas em torno de 0 e com suporte finito, ou com suporte infinito desde que $\psi(t) \rightarrow 0$ rapidamente quando $|t| \rightarrow \infty$.

Definição 3.2. Uma ondaleta é dita possuir $Q \geq 1$ momentos nulos se

$$
\begin{aligned}
\int_{\mathbb{R}} t^{p} \psi(t) d t & =0, \quad p=0,1, \ldots, Q-1 \\
\int_{\mathbb{R}} t^{Q} \psi(t) d t & \neq 0 .
\end{aligned}
$$

Exemplos de ondaletas incluem as derivadas da densidade normal padrão $\psi(t)=\frac{d^{n}}{d t^{n}}(2 \pi)^{-1 / 2} e^{-t^{2} / 2}$, sendo a segunda derivada conhecida como chapéu mexicano (mexican hat) devido a forma que esta assume; a ondaleta de Haar dada por $\psi(t)=\mathbb{I}_{[0,1 / 2)}(t)-\mathbb{I}_{[1 / 2,1)}(t)$, onde $\mathbb{I}_{A}(\cdot)$ é a função indicadora do conjunto $A$, além de outras como as ondaletas de Daubechies e Meyer (Doukhan et al., 2003).

Definição 3.3 (Coeficiente ondaleta). Dada uma escala $b \in \mathbb{R}_{+}^{*}$ e uma localização $k \in \mathbb{R}, o$ coeficiente ondaleta de um processo $\left\{X_{t}, t \in \mathbb{R}\right\}$ é definido por

$$
d(b, k)=\frac{1}{\sqrt{b}} \int_{\mathbb{R}} \psi\left(\frac{t}{b}-k\right) X_{t} d t
$$

onde $\psi: \mathbb{R} \rightarrow \mathbb{R}$ é uma ondaleta.

\footnotetext{
${ }^{1}$ http://math.bu.edu/people/murad/methods/index.html

${ }^{2}$ http://samm.univ-paris1.fr/Sofwares-Logiciels
} 
Se a ondaleta $\psi$ decai suficientemente rápido quando $|t|$ cresce e satisfaz certas condições cuja formulação depende do processo $\left\{X_{t}, t \in \mathbb{R}\right\}$ analisado, então os coeficientes ondaleta são v.a.'s bem definidas.

Na prática, como o processo é observado através de uma série $x_{1}, \ldots, x_{n}$, o coeficiente ondaleta de uma coleção de escalas inteiras positivas $b_{j},(j=1, \ldots, J)$ é aproximado por

$$
e\left(b_{j}, k\right)=\frac{1}{\sqrt{b_{j}}} \sum_{i=1}^{n} \psi\left(\frac{i}{b_{j}}-k\right) x_{i},
$$

onde $k \in \mathbb{Z}$. Tal aproximação é boa somente se $b_{j}$ e $\left[n / b_{j}\right]$ forem suficientemente grandes.

Em nosso contexto, se $\left\{X_{t}, t \in \mathbb{R}\right\}$ for um processo de Hermite então, sob certas condições de regularidade, a variância de $d(b, k)$ é uma função potência de $b$ com expoente $1-2 H$. Se $\left\{X_{t}, t \in \mathbb{R}\right\}$ for um movimento estável fracionário, então a variância de $d(b, k)$ é uma função potência de $b$ com expoente $-2 d \operatorname{com} d=H-\frac{1}{\alpha}$. Assim, se considerarmos a seguinte aproximação de $\operatorname{Var}[d(b, k)]$

$$
S_{n}\left(b_{j}, k\right)=\frac{1}{\left[n / b_{j}\right]} \sum_{k=1}^{\left[n / b_{j}\right]} e\left(b_{j}, k\right)^{2},
$$

então um estimador $\hat{H}_{W a}$ de $H$ pode ser obtido pela regressão linear de $\log S_{n}\left(b_{j}, k\right)$ em $\log \left(b_{j}\right)$, $j=1, \ldots, J$.

A principal dificuldade que surge na aplicação desse método é a escolha da maior escala $b_{j}$ para que a aproximação $e\left(b_{j}, k\right)$ seja boa. Veitch et al. (2003) propõem um método heurístico para a escolha dessas escalas, enquanto Bardet et al. (2008) provam a consistência do método em um contexto semiparamétrico Gaussiano quando as escalas são escolhidas com base no estudo do variograma da série observada. Este resultado é estendido para processos lineares em Bardet e Bibi (2012).

O método de ondaletas está implementado no software R-gui através do pacote fArma, enquanto implementações em linguagem MATLAB podem ser obtidas gratuitamente em http://samm. univ-paris1.fr/Sofwares-Logiciels.

\subsection{Variação- $p$}

O estudo de variações de um processo estocástico é importante tanto do ponto de vista probabilístico quanto estatístico. Para processos autossimilares o estudo de suas variações constitui uma ferramenta para construir estimadores para o parâmetro de autossimilaridade e consequentemente para o índice de memória.

Antes de definirmos a variação- $p$ amostral, vamos introduzir o conceito de filtros discretos que será utilizado para construir estatísticas resumo para serem utilizadas no método ABC.

Definição 3.4 (Filtros discretos). Um filtro $f$ com comprimento $l \in \mathbb{N}$ e ordem $k \in \mathbb{N}(k \neq 0)$ é um vetor $f=\left\{f_{0}, f_{1}, \ldots, f_{l}\right\}$ que satisfaz

$$
\begin{aligned}
& \sum_{q=0}^{l} f_{q} q^{r}=0, \quad \text { para } 0 \leq r \leq k-1, \quad r \in \mathbb{Z}, \\
& \sum_{q=0}^{l} f_{q} q^{k} \neq 0
\end{aligned}
$$

com a convenção $0^{0}=1$.

Isto significa dizer que filtros discretos, assim como as ondaletas apresentadas na subseção anterior, possuem $k$ momentos nulos. 
Como na prática o processo é observado em tempo discreto, temos que se $X_{1}, \ldots, X_{n}$ for uma trajetória amostral de comprimento $n$ do processo $\left\{X_{t}, t \in \mathbb{R}\right\}$, então a série filtrada, denotada por $\mathbf{X}(f)=\left\{\mathbf{X}_{i}(f), i=l+1, \ldots, n\right\}$, é dado pela convolução da série com o filtro, isto é

$$
\mathbf{X}_{i}(f)=\sum_{q=0}^{l} f_{q} X_{i-q}, \text { para } i=l+1, \ldots, n .
$$

Alguns exemplos de filtros discretos são os filtros de diferença finita, conhecidos também como operadores diferença ou retrocesso, isto é

1. para $f=\{1,-1\}$ (filtro de diferença finita de ordem 1),

$$
\mathbf{X}_{i}(f)=X_{i}-X_{i-1}:=\Delta X_{i} .
$$

2. Para $f=\{1,-2,1\}$ (filtro de diferença finita de ordem 2),

$$
\mathbf{X}_{i}(f)=X_{i}-2 X_{i-1}+X_{i-2}=\Delta\left(\Delta X_{i}\right):=\Delta^{2} X_{i} .
$$

3. Em geral, um processo submetido a um filtro de diferença finita de ordem $k$ é dado por

$$
\mathbf{X}_{i}(f)=\Delta^{k-1}\left(\Delta X_{i}\right):=\Delta^{k} X_{i}
$$

Definição 3.5 (Variação-p amostral). Sejam $X_{1}, \ldots, X_{n}$ uma trajetória amostral do processo estocástico $\left\{X_{t}, t \in \mathbb{R}\right\}$ e $f=\left\{f_{0}, \ldots, f_{l}\right\}$ um filtro de comprimento l e ordem $k$. A variação-p amostral da série filtrada $X_{l+1}(f), \ldots, X_{n}(f)$ é definida por

$$
V_{n}^{(p)}(f):=\sum_{i=l+1}^{n}\left|X_{i}(f)\right|^{p} .
$$

A variação- $p$ é uma generalização dos conceitos de variação total $(p=1)$ e variação quadrática $(p=2)$, amplamente aplicados nas áreas de física, matemática e engenharia (Burnecki e Weron , 2010). No caso de processos autossimilares, a variação quadrática pode ser utilizada para estimar o parâmetro de autossimilaridade (Chronopoulou e Viens, 2009; Chronopoulou et al., 2008, 2009).

Seja $f=\{1,-1\}$ e considere a variação- $p$ de lag $m$ definida por

$$
V_{m}^{(p)}=\sum_{i=1}^{[n / m]-1}\left|X_{(i+1) m}-X_{i m}\right|^{p} .
$$

Dada uma trajetória amostral $X_{1}, \ldots, X_{n}$ de um fSm e $n / m$ suficientemente grande, Burnecki et al. (2012) argumentam que:

- Se $\alpha=2$ (caso fBm), então $V_{m}^{(p)} \sim m^{H p-1}$;

- se $1<\alpha<2$ e $H>\frac{1}{\alpha}$, então $V_{m}^{(p)} \sim m^{H p-1}$;

- se $1<\alpha<2$ e $H<\frac{1}{\alpha}$, então $V_{m}^{(p)} \sim m^{p\left(H-\frac{1}{\alpha}\right)}$.

Quando $H>\frac{1}{\alpha}$, ou seja, quando o fSm é um processo com memória longa, temos que a variação- $p$ amostral de lag $m$ é uma função crescente de $m$ se $p>\frac{1}{H}$ e decrescente quando $p<\frac{1}{H}$.

A idéia do método da variação- $p$ de lag $m$ é encontrar $p=\frac{1}{H}$ para o qual $V_{m}^{(p)}$, como função de $m$, muda seu comportamento monótono tornando-se constante. Burnecki et al. (2012) propõem calcular a diferença ao quadrado entre $V_{1}^{(p)}$ e $V_{m}^{(p)}$ para um valor grande de $m$, mas este não deve ser muito grande para garantir que $n / m$ seja grande (na prática $m=8$ é suficiente). Essas diferenças 
devem ser calculadas para vários valores de $p$, por exemplo, $p \in\left\{\frac{1}{0.01}, \frac{1}{0.02}, \ldots, \frac{1}{0.99}\right\}$. A menor diferença define o estimador $\hat{p}$ e consequentemente $\hat{H}=\frac{1}{\hat{p}}$.

Uma característica importante desse método é que este pode estimar o parâmetro de autossimilaridade $H$ sem a necessidade de conhecer o valor do parâmetro de estabilidade $\alpha$. No entanto, é importante conhecer o valor de $\alpha$ para determinar se o processo possui memória longa ou não. Além disso, veremos nas aplicações que as estimativas obtidas para $H$ através desse método parecem sofrer influência do valor de $\alpha$ do processo adjacente. Essa influência desaparece conforme $\alpha$ se aproxima de 2 (caso normal).

\subsection{Método MSD}

O método do deslocamento quadrático médio amostral, ou simplesmente MSD (sample meansquared displacement), foi apresentado em Burnecki e Weron (2010) como um método para estimar o parâmetro de memória $d=H-\frac{1}{\alpha}$ do movimento estável fracionário.

Dado uma trajetória amostral $X_{1}, \ldots, X_{n}$ de comprimento $n$ de um fSm com $H \in(0,1)$ e $\alpha \in(0,2]$, o deslocamento quadrático médio amostral $M_{n}(k)$ de lag $k$ é definido como

$$
M_{n}(k)=\frac{1}{n-k} \sum_{i=1}^{n-k}\left(X_{i+k}-X_{i}\right)^{2} .
$$

Para $n$ suficientemente grande, Burnecki e Weron (2010) mostram que $M_{n}(k) \sim k^{2 d+1}$, onde $d=H-\frac{1}{\alpha}$. Assim, o método MSD pode ser implementado da seguinte maneira:

(i) Calcule $M_{n}(k)$ para alguns valores de $k$ (os 10 primeiros são suficientes);

(ii) ajuste uma reta de regressão aos pontos $\left(\log k, \log M_{n}(k)\right)$ e obtenha sua inclinação $a$;

(iii) obtenha a estimativa de $d$ através de $\hat{d}=(a-1) / 2$.

Podemos concluir, a partir dessa análise, que se $\hat{d}>0$ então o fSm que gerou os dados possui memória longa. Observe que para estimar o parâmetro de autossimilaridade $H$, devemos conhecer ou estimar o valor do parâmetro de estabilidade $\alpha$. 


\section{Capítulo 4}

\section{Computação Bayesiana Aproximada}

Muitas aplicações estatísticas têm como objetivo inferir sobre modelos estocásticos através de observações parciais do mesmo. Em geral é fácil obter simulações desses modelos, mas o cálculo da verossimilhança dos dados pode ser impraticável.

Iniciamos esta seção com os seguintes questionamentos: $\mathrm{O}$ que podemos fazer quando a função de verossimilhança é impossível, ou computacionalmente custosa de ser calculada? Uma vez que métodos de Monte Carlo via cadeias de Markov (MCMC) não podem ser implementados, por serem dependentes da função de verossimilhança, devemos desistir completamente da abordagem Bayesiana?

A classe de métodos conhecida como computação Bayesiana aproximada, ou simplesmente ABC (approximate Bayesian computation) surge como uma alternativa para esses casos problemáticos, buscando formas de aproximar a distribuição a posteriori de interesse pela distribuição a posteriori condicionada a funções da amostra observada.

Os métodos ABC tiveram sua gênese com a proposta simples, embora muito limitada, de Rubin (1984), passando a ganhar maior visibilidade no final da década de 90 com os trabalhos de Tavaré et al. (1997) e Pritchard et al. (1999), ambos na área de genética populacional. A partir daí surgiram vários trabalhos relacionados ao emprego do $\mathrm{ABC}$ e suas características, e nota-se um crescente interesse no uso desses métodos nas mais diversas áreas como em epidemiologia (McKinley et al., 2009), inferência para extremos (Bortot et al., 2007), sistemas dinâmicos (Toni et al., 2009) e campos aleatórios de Gibbs (Grelaud et al., 2009).

Suponha que $\mathcal{M}$ seja um modelo para um conjunto de dados $\mathcal{D}$, em um espaço amostral $\mathcal{X}$ e que $\theta$, pertencente a um espaço paramétrico $\Theta$, seja um vetor de parâmetros deste modelo. Suponha também que $\pi$ seja uma distribuição a priori para $\theta$ e $\mathbb{P}(\mathcal{D} \mid \theta)$ a verossimilhança dos dados. Lembremos que a distribuição a posteriori de $\theta$, denotada por $f(\theta \mid \mathcal{D})$, atualiza a distribuição a priori após observar os dados. Esta é definida por

$$
f(\theta \mid \mathcal{D})=\frac{\mathbb{P}(\mathcal{D} \mid \theta) \pi(\theta)}{\int \mathbb{P}(\mathcal{D} \mid \theta) \pi(\theta) d \theta}=\frac{\mathbb{P}(\mathcal{D} \mid \theta) \pi(\theta)}{\mathbb{P}(\mathcal{D})} .
$$

Um dos problemas mais comuns na área de inferência Bayesiana é o cálculo da distribuição a posteriori (4.1), pois na maioria das aplicações não é possível obter analiticamente tal distribuição. Por outro lado, quando tais posterioris podem ser obtidas analiticamente, seu cálculo pode ser computacionamente dispendioso ou até mesmo impossível. Uma alternativa para solucionar este tipo de problema é recorrer às técnicas de simulação estocástica, técnicas estas que promoveram uma revolução no emprego da inferência Bayesiana em problemas complexos ou insolúveis analiticamente sob o ponto de vista Bayesiano.

Os métodos de Monte Carlo, ou MCMC são amplamente utilizados para produzir amostras de uma distribuição a posteriori de interesse. Uma visão geral dessas técnicas pode ser obtida em Gamerman e Lopes (2006) e Robert e Casella (2013). Partiremos da abordagem mais simples de simulação estocástica; o método de rejeição 


\section{Algoritmo A: Rejeição simples}

A1: Simule $\theta^{*}$ da priori $\pi($.$) ,$

A2: aceite $\theta^{*}$ com probabilidade $h=\mathbb{P}\left(\mathcal{D} \mid \theta^{*}\right)$; retorne a A1.

A repetição do algoritmo A produz uma amostra de valores de $\theta$ cuja distribuição adjacente é $f(\theta \mid \mathcal{D})$. De fato, observe que

$$
\begin{aligned}
\mathbb{P}(\theta \leq x \mid \theta \text { é aceito }) & =\frac{\mathbb{P}(\theta \leq x, \theta \text { é aceito })}{\mathbb{P}(\theta \text { é aceito })}=\frac{\int_{-\infty}^{x} \mathbb{P}(\mathcal{D} \mid \theta) \pi(\theta) d \theta}{\int_{-\infty}^{\infty} \mathbb{P}(\mathcal{D} \mid \theta) \pi(\theta) d \theta} \\
& =\int_{-\infty}^{x} f(\theta \mid \mathcal{D}) d \theta=F(x \mid \mathcal{D}) .
\end{aligned}
$$

Pode-se aumentar a eficiência do algoritmo A caso seja conhecido um valor $M>0$ tal que $\mathbb{P}(\mathcal{D} \mid \theta) \leq M$ para qualquer conjunto de dados $\mathcal{D}$, bastando substituir $h$ por $h / M$. Logo, se $\hat{\theta}$ é o estimador de máxima verossimilhança de $\theta$, então $M=\mathbb{P}(\mathcal{D} \mid \hat{\theta})$.

Observe que para utilizar o algoritmo A devemos conhecer e calcular a verossimilhança $\mathbb{P}(\mathcal{D} \mid \theta)$. No entanto, existem modelos probabilísticos complexos para os quais o cálculo da verossimilhança é impossível ou computacionalmente proibitivo. Rubin (1984) apresenta uma alternativa para resolver este tipo de problema, embora tal alternativa seja limitada aos espaços amostrais discretos. Trata-se do algoritmo de rejeição sem verossimilhança, dado pelo algoritmo B a seguir:

Algoritmo B: Rejeição sem verossimilhança
B1: Simule $\theta^{*}$ da priori $\pi()$,
B2: simule $\mathcal{D}^{\prime}$ do modelo $\mathcal{M}$ com o parâmetro $\theta^{*}$ simulado em B1,
B3: aceite $\theta^{*}$ se $\mathcal{D}^{\prime}=\mathcal{D}$; retorne a B1.

Neste caso, o valor $\theta^{*}$ selecionado é distribuído segundo $f(\theta \mid \mathcal{D})$ (veja Rubin (1984) e Diggle e Gratton (1984)). Observe que no algoritmo B não há a necessidade de se conhecer a verossimilhança, bastando apenas que seja possível, e fácil, simular dados do modelo $\mathcal{M}$. Note também que caso a probabilidade $\mathbb{P}(\mathcal{D})$ seja pequena, a probabilidade de aceitação será baixa, uma vez que tal probabilidade é proporcional a $\mathbb{P}(\mathcal{D})$. Uma alternativa aos casos onde a taxa de aceitação é muito baixa, ou quando os dados são contínuos, é recorrer ao seguinte algoritmo:

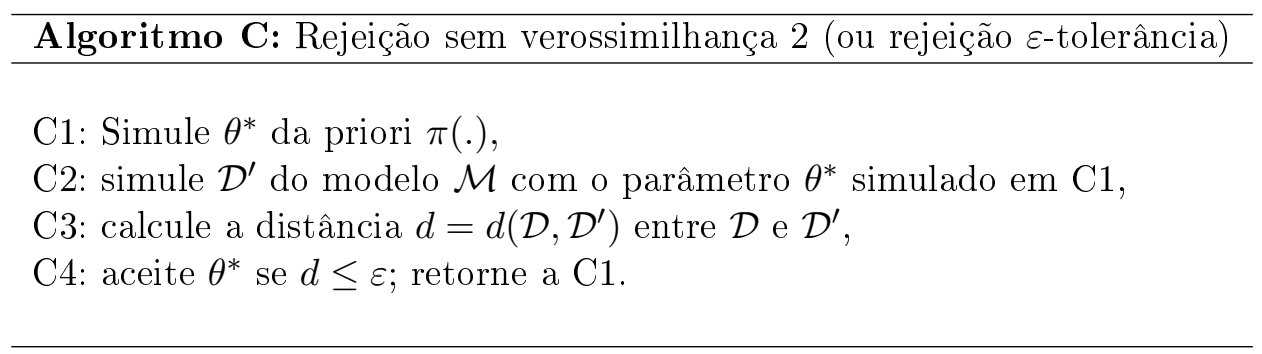

Para utilizar o algoritmo $\mathrm{C}$ devemos selecionar uma medida de discrepância $d$ em $\mathcal{X}$ e uma tolerância $\varepsilon>0$. Quando $\varepsilon \rightarrow \infty$ o algoritmo simplesmente produz uma amostra da distribuição a priori. Por outro lado, se $\varepsilon=0$ então $\mathcal{D}=\mathcal{D}^{\prime}$ e assim, os valores simulados aceitos serão realizações de $f(\theta \mid \mathcal{D})$ segundo o algoritmo B.

Como $\varepsilon>0$ na prática, o algoritmo $\mathrm{C}$ amostra da marginal em $\mathcal{D}^{\prime}$ da seguinte distribuição conjunta 


$$
f_{\varepsilon}\left(\theta, \mathcal{D}^{\prime} \mid \mathcal{D}\right) \propto \pi(\theta) f\left(\mathcal{D}^{\prime} \mid \theta\right) \mathbb{I}_{A_{\varepsilon, \mathcal{D}}}\left(\mathcal{D}^{\prime}\right),
$$

onde $\mathbb{I}_{B}\left(\right.$.) denota a função indicadora do conjunto $B$ e $A_{\varepsilon, \mathcal{D}}=\left\{\mathcal{D}^{\prime} \in \mathcal{X}: d\left(\mathcal{D}, \mathcal{D}^{\prime}\right) \leq \varepsilon\right\}$. Assim, para $\varepsilon$ suficientemente pequeno, teremos uma aproximação para a distribuição a posteriori, a saber

$$
f_{\varepsilon}(\theta \mid \mathcal{D})=\int f_{\varepsilon}\left(\theta, \mathcal{D}^{\prime} \mid \mathcal{D}\right) d \mathcal{D}^{\prime} \approx f(\theta \mid \mathcal{D}) .
$$

A escolha de $\varepsilon$ induz um problema de "trade-off" entre custo computacional e precisão. Voltaremos a comentar sobre a escolha de $\varepsilon$ posteriormente nos exemplos.

Quando os dados possuem dimensão alta, as abordagens apresentadas até agora podem tornarse impraticáveis. Além disso, os algorítmos B e C não são úteis quando os dados são contínuos, já que eventos do tipo $\left\{\mathcal{D}=\mathcal{D}^{\prime}\right\}$ possuem medida de probabilidade nula. Pritchard et al. (1999) propuseram o que muitos autores consideram o primeiro algoritmo genuinamente $\mathrm{ABC}$, cuja ideia é comparar os dados $\mathcal{D}$ e $\mathcal{D}^{\prime}$ através de estatísticas resumo adequadas. O emprego de estatísticas resumo visa eliminar problemas de dimensionalidade ou problemas da natureza dos dados como, por exemplo, quando os dados são séries temporais. Dado um conjunto $T=\left(T_{1}, \ldots, T_{p}\right)$ de $p$ estatísticas, o algoritmo proposto em Pritchard et al. (1999) é dado por:
Algoritmo D: ABC 1
D1: Simule $\theta^{*}$ da priori $\pi($.$) ,$
D2: simule $\mathcal{D}^{\prime}$ do modelo $\mathcal{M}$ com o parâmetro $\theta^{*}$ simulado em D1 e calcule $T^{\prime}=T\left(\mathcal{D}^{\prime}\right)$,
D3: calcule a distância $d=d\left(T, T^{\prime}\right)$ entre $T$ e $T^{\prime}$,
D4: aceite $\theta^{*}$ se $d \leq \varepsilon$; retorne a D1.

A constante normalizadora $\mathbb{P}(T)$ é tipicamente maior que $\mathbb{P}(\mathcal{D})$, o que resulta em um número maior de aceitações dos valores simulados pelo algoritmo D.

Diferente do algoritmo $\mathrm{C}$, que amostra de $f\left(\theta \mid d\left(\mathcal{D}, \mathcal{D}^{\prime}\right) \leq \varepsilon\right)$, o algortimo D amostra da distribuição de $f\left(\theta \mid d\left(T, T^{\prime}\right) \leq \varepsilon\right)$. Lembremos que se $T$ é uma estatística suficiente para $\theta$, então $\mathbb{P}(\mathcal{D} \mid T, \theta)$ independe de $\theta$ e consequentemente $f(\theta \mid T)=f(\theta \mid \mathcal{D})$. Logo, quando $\varepsilon \rightarrow 0$, temos que $f\left(\theta \mid d\left(T, T^{\prime}\right) \leq \varepsilon\right) \rightarrow f(\theta \mid T)$ que é igual a $f(\theta \mid \mathcal{D})$ se $T$ for uma estatística suficiente para $\theta$.

Vale mencionar que na prática são raros os casos onde uma estatística suficiente esteja disponível para problemas que demandam o uso de métodos ABC. Devemos usar o conhecimento sobre o modelo que será utilizado para propor um conjunto de estatísticas capaz de manter boa parte da informação sobre $\theta$ contida nos dados. Assim, se $T$ não é uma estatística suficiente, o máximo que se pode esperar do algoritmo $\mathrm{D}$ é que $\theta^{*} \sim f(\theta \mid T)$ e não $\theta^{*} \sim f(\theta \mid \mathcal{D})$. Caso a estatística $T$ seja ancilar a situação se agrava, uma vez que toda a informação sobre $\theta$ contida em $\mathcal{D}$ é perdida, e consequentemente $\theta^{*} \sim \pi(\theta)$. Logo, métodos ABC como o algoritmo D usam estatísticas insuficientes buscando que $f\left(\theta \mid d\left(T, T^{\prime}\right) \leq \varepsilon\right) \approx f(\theta \mid \mathcal{D})$ para $\varepsilon$ suficientemente pequeno, e essa aproximação pode ser muito ruim se a estatística $T$ não for selecionada com cautela.

No decorrer deste trabalho, iremos chamar de representativas ou potencialmente informativas as estatísticas que preservam alguma informação sobre $\theta$ contida nos dados. Essa importante característica das estatísticas será observada com mais clareza nos exemplos e nas aplicações.

Exemplo 1 - Distribuições $\alpha$-estáveis. Considere estimar o parâmetro de localização de uma distribuição $\alpha$-estável (apresentada na Definição 2.6). Para isso, considere um conjunto de dados de $S(\alpha, \beta, \gamma, \delta)$ obtidos por simulação, assumindo que $\alpha, \beta$ e $\gamma$ são conhecidos e que $\delta$ seja desconhecido. Usaremos o algoritmo D para inferir sobre $\delta$.

Como $\delta$ é simplesmente um parâmetro de localização, temos que a média amostral ou a mediana amostral são escolhas óbvias para estatísticas resumo. Podemos observar o quão representativas essas estatísticas são para o parâmetro de interesse $\delta$ através de simulações do modelo. Simulamos 10000 
valores de $\delta$ uniformemente no intervalo $(-100,100)$ e, para cada valor de $\delta$, simulamos uma amostra do modelo $S(1.5,0.3,1, \delta)$. Para cada amostra simulada, calculamos sua média e sua mediana para, em seguida, plotar os valores de $\delta$ simulados contra os valores simulados dessas estatísticas. A Figura 4.1(a,b) apresenta esses gráficos, onde fica claro a capacidade que essas estatísticas possuem para discriminar valores diferentes de $\delta$. Neste caso a mediana é mais precisa, pois a média é influenciada pelos outliers que são comuns nos dados, já que as distribuições estáveis com $\alpha<2$ possuem variância infinita. Note que se fosse utilizado o desvio-padrão amostral como estatística resumo (o que seria um absurdo), não seria possível extrair informação alguma sobre $\delta$ (Figura 4.1(c)). Apesar de óbvio, esse recurso é muito útil e importante para selecionar boas estatísticas resumo para o algoritmo D, mas este só é útil se as estatísticas utilizadas tiverem no máximo dimensão 2 para cada parâmetro.

(a) $\mathrm{T}(\mathrm{x})=\overline{\mathrm{x}}$

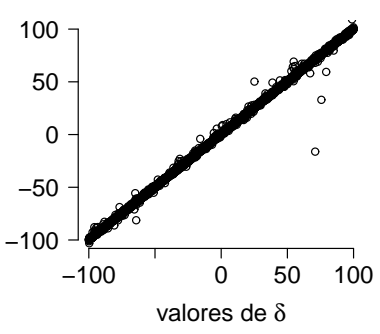

(c) $T(x)=d p(x)$

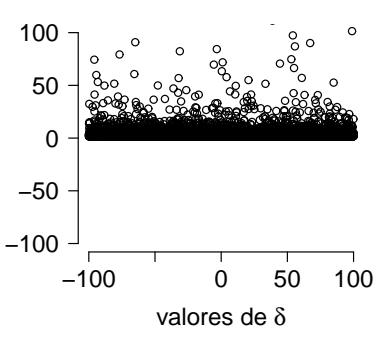

(b) $\mathrm{T}(\mathrm{x})=$ mediana $(\mathrm{x})$

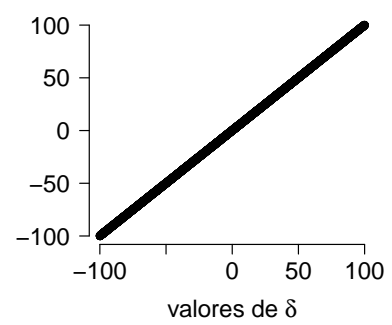

Figura 4.1: Estudo da média, mediana e desvio-padrão como estatísticas resumo para o parâmetro de localização $\delta$ da distribuição $S(1.5,0.3,1, \delta)$.

Assuma que a amostra aleatória observada de $S(1.5,0.3,1, \delta)$ tenha tamanho $n=100$ e que o valor nominal de $\delta$ seja 2. Considere, a priori, que $\delta \sim N\left(0, \sigma^{2}\right)$ com $\sigma=5$.

Usaremos o algoritmo D com $N=10^{4}$ repetições e tomaremos a distância Euclidiana na etapa D3. Além disso, vamos escolher $\varepsilon$ de modo a aceitar $1 \%$ dos valores simulados (nessas condições, $\varepsilon \approx 0.0039$ ). A Figura 4.2 mostra a curva da densidade a priori para $\delta$ e o histograma da amostra obtida através do algoritmo D cuja média é igual a 1.842 e o desvio-padrão 0.432 .

Vamos investigar agora como ficam os resultados variando o valor da tolerância $\varepsilon$. Para isso, repetimos o estudo feito anteriormente nas mesmas condições e para a mesma amostra, variando apenas a escolha de $\varepsilon$. A Tabela 4.1 fornece o valor de $\varepsilon$ escolhido, o tamanho da amostra aceita de valores de $\delta$, bem como média e desvio-padrão dessas amostras. Note que quando $\varepsilon$ cresce, a média aproxima-se da média da distribuição a priori, que neste caso é 0 .

\begin{tabular}{ccc}
\hline \hline Valor de $\varepsilon$ & Tamanho da amostra de $\delta$ & média (desvio-padrão) \\
\hline 0.0001 & 10 & $1.6875(0.5286)$ \\
0.0039 & 100 & $1.8424(0.4321)$ \\
0.4316 & 1000 & $1.8184(0.5929)$ \\
79.8296 & 9000 & $0.6477(4.0374)$ \\
\hline \hline
\end{tabular}

Tabela 4.1: Estudo do impacto da escolha de $\varepsilon$ na inferência sobre $\delta$ usando o algoritmo D. 


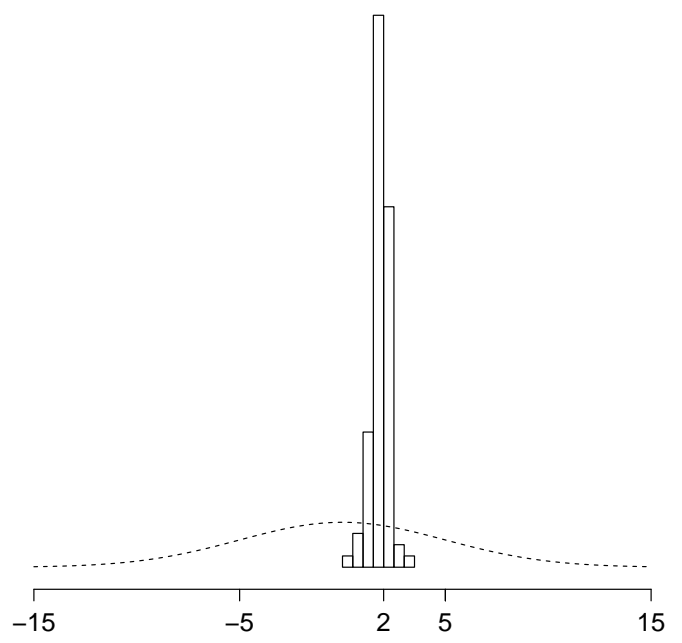

Figura 4.2: Histograma dos valores de $\delta$ obtidos pelo algoritmo D para uma amostra de tamanho 100 da distribuição $S(1.5,0.3,1, \delta=2)$. A curva tracejada representa a função de densidade a priori que neste caso é $N(0,25)$.

Observação 4.1 (Escolha de $\varepsilon$ ). Ratmann et al. (2009) e Fearnhead e Prangle (2012) propõem técnicas para obtenção de $\varepsilon$, mas em geral este valor é escolhido como um pequeno percentil das distâncias simuladas no passo D3 do algoritmo D (Marin et al., 2012) (usualmente 1\% ou 10\%). Deste modo é possivel obter previamente o número de repetiçôes necessárias para se obter um tamanho de amostra adequado, isto é, se $N$ é o número de repetiçóes do algoritmo e $\varepsilon$ é o percentil de $q \%$ das distâncias simuladas no passo $D 3$ do algoritmo $D$, então a amostra obtida pelo mesmo terá tamanho $N q / 100$.

Com o intuito de estudar a performance do algoritmo D para diferentes estatísticas resumo, realizamos 100 simulações de Monte Carlo para estimar $\delta$ considerando as seguintes estatísticas:

(i) Média amostral,

(ii) mediana amostral,

(iii) percentis empíricos com valores em $\{5 \%, 15 \%, 25 \%, \ldots, 85 \%, 95 \%\}$,

(iv) todas as estatísticas acima conjuntamente.

Novamente, em todos os casos $\varepsilon$ foi tomado de modo a aceitar $1 \%$ das $N=10^{4}$ repetições do algoritmo $\mathrm{D}$ considerando a priori que $\delta \sim N(0,25)$. O estimador utilizado foi a média a posteriori e os boxplots das estimativas obtidas por cada abordagem são dados na Figura 4.3, enquanto média e desvio-padrão das estimativas sob cada abordagem são dados na Tabela 4.2. Observe que dentre as quatro abordagens, a melhor em termos de viés e precisão é a que utiliza apenas a mediana amostral como estatística resumo, enquanto a que utiliza apenas a média amostral é a pior. Além disso, esse estudo ilustra o fato de que um conjunto com muitas estatísticas resumo nem sempre torna a performance do algoritmo D melhor.

Por último, considere a análise do efeito da escolha da priori. Novamente, foram realizadas 100 simulações de Monte Carlo do modelo $S(1.5,0.3,1, \delta=2)$ e $\delta$ foi estimado usando o algoritmo D. Desta vez a mediana amostral foi utilizada como estatística resumo e $\varepsilon$ foi escolhido de modo a aceitar 100 das $N=10^{4}$ repetições do algoritmo. Assuma a priori que $\delta \sim N\left(\mu, \sigma^{2}\right)$ com $\mu=0$ e $\sigma \in\{1,5,10,20\}$ e $\delta \sim N(-2,1)$, sendo esta última priori uma má especificação proposital. Os 


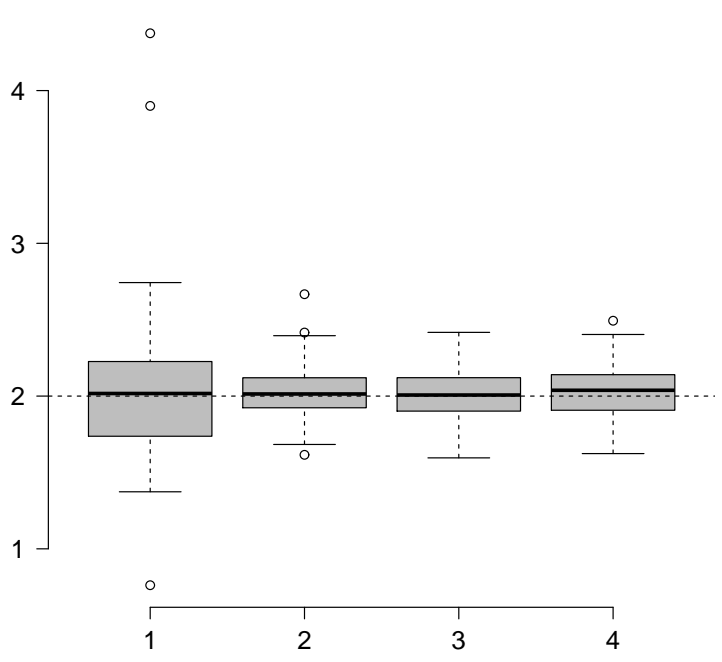

Figura 4.3: Boxplots das médias a posteriori obtidas para $\delta$ usando o algoritmo $D$ em 100 simulações de Monte Carlo. Os números $1-4$ no eixo horizontal indicam as abordagens $(i)-(i v)$.

\begin{tabular}{cc}
\hline \hline Abordagem & média (desvio-padrão) \\
\hline (i) & $2.0257(0.4505)$ \\
(ii) & $2.0246(0.1602)$ \\
(iii) & $2.0068(0.1712)$ \\
(iv) & $2.0286(0.1732)$ \\
\hline \hline
\end{tabular}

Tabela 4.2: Média e desvio-padrão das estimativas (média a posteriori) obtidas para $\delta$ usando o algoritmo D em 100 simulações de Monte Carlo segundo as abordagens (i) - (iv).

boxplots da Figura 4.4 representam as estimatívas obtidas para cada priori assumida, enquanto a Tabela 4.3 nos fornece a média e o desvio padrão da distribuição amostral dessas estimativas.

\begin{tabular}{cc}
\hline \hline Priori & média (desvio-padrão) \\
\hline$N(0,1)$ & $1.9123(0.1785)$ \\
$N(0,25)$ & $1.9721(0.1575)$ \\
$N(0,100)$ & $2.0100(0.1716)$ \\
$N(0,400)$ & $1.9834(0.1643)$ \\
$N(-2,1)$ & $0.6174(0.0520)$ \\
\hline \hline
\end{tabular}

Tabela 4.3: Média e devio-padrão das estimativas (média a posteriori) obtidas para $\delta$ usando o algoritmo $D$ em 100 simulações de Monte Carlo utilizando diferentes distribuições a priori para $\delta$.

Note que não há muita diferença nas estimativas obtidas quando utilizamos prioris normais centradas em zero, pois todas conseguem gerar valores próximos do valor nominal de $\delta$. No entanto, considerando a priori que $\delta \sim N(-2,1)$, o algoritmo $\mathrm{D}$ tende a aceitar valores na cauda desta priori, e como a distribuição normal possui caudas leves, o algortimo não consegue simular valores próximos de 2. Esta sensibilidade a uma má especificação da distribuição a priori é um dos problemas do algoritmo D.

Os métodos de rejeição apresentados até o momento possuem diversas vantagens, como a simplicidade, facilidade de implementação e o fato de gerar observações independentes. No entanto, 


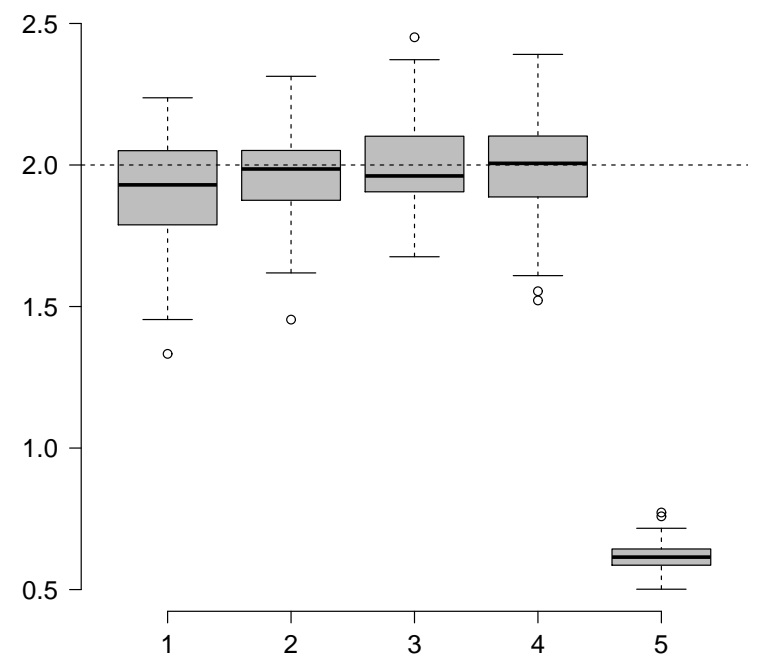

Figura 4.4: Boxplots das médias a posteriori obtidas para $\delta$ usando o algoritmo $D$ em 100 simulações de Monte Carlo. Os números $1-5$ no eixo horizontal indicam cada uma das 5 prioris assumidas para $\delta$.

como vimos no final do exemplo anterior, estes métodos perdem eficiência quando há conflito de informação. Para superar este tipo de problema, Marjoram et al. (2003) propõem incluir uma etapa do tipo Metropolis-Hastings ao algoritmo D, tornando-o assim mais eficiente ao custo de se obter observações correlacionadas.

Recordemos brevemente o que o algoritmo de Metropolis-Hastings faz (para maiores detalhes veja Gamerman e Lopes (2006) ou Robert e Casella (2013)). Tal algoritmo gera observações de $f(\theta \mid \mathcal{D})$ da seguinte maneira:

MH1: Se o estado atual é $\theta$, mova para o estado $\theta^{\prime}$ de acordo com o kernel de transição $q\left(\theta \rightarrow \theta^{\prime}\right)$,

MH2: calcule

$$
h=\min \left\{1, \frac{\mathbb{P}\left(\mathcal{D} \mid \theta^{\prime}\right) \pi\left(\theta^{\prime}\right) q\left(\theta^{\prime} \rightarrow \theta\right)}{\mathbb{P}(\mathcal{D} \mid \theta) \pi(\theta) q\left(\theta \rightarrow \theta^{\prime}\right)}\right\}
$$

MH3: mova para $\theta^{\prime}$ com probabilidade $h$, caso contrário permaneça no estado $\theta$; vá para MH1.

Sob certas condições de regularidade, o algoritmo acima gera estados que formam uma cadeia de Markov cuja distribuição estacionária é $f(\theta \mid \mathcal{D})$. Novamente, o algoritmo de Metropolis-Hastings requer o cálculo da função de verossimilhança em cada repetição. Este algoritmo tende a aceitar mais valores que o algoritmo A, mas o preço dessa eficiência é a dependência que existe entre os valores simulados.

Com base no algoritmo acima e no algoritmo D, Marjoram et al. (2003) propõem o seguinte algoritmo ABC:

\footnotetext{
Algoritmo E: MCMC-ABC ou MCMC sem verossimilhança

E1: Se o estado atual é $\theta$, mova para o estado $\theta^{\prime}$ de acordo com o kernel de transição $q\left(\theta \rightarrow \theta^{\prime}\right)$,

E2: simule $\mathcal{D}^{\prime}$ do modelo $\mathcal{M}$ com o parâmetro $\theta^{\prime}$,

E3: se $\mathcal{D}^{\prime}=\mathcal{D}$, vá para $\mathrm{E} 4$, caso contrário permaneça no estado $\theta$ e vá para $\mathrm{E} 1$,

E4: calcule $h=h\left(\theta, \theta^{\prime}\right)=\min \left\{1, \frac{\pi\left(\theta^{\prime}\right) q\left(\theta^{\prime} \rightarrow \theta\right)}{\pi(\theta) q\left(\theta \rightarrow \theta^{\prime}\right)}\right\}$,

E5: aceite $\theta^{\prime}$ com probabilidade $h$, caso contrário permaneça em $\theta$; vá para E1.
} 
A distribuição estacionária da cadeia formada pelo algoritmo E é $f(\theta \mid \mathcal{D})$. De fato, denote por $r\left(\theta \rightarrow \theta^{\prime}\right)$ o mecanismo de transição da cadeia e suponha que $\theta^{\prime} \neq \theta$ satisfaz a condição

$$
\frac{\pi\left(\theta^{\prime}\right) q\left(\theta^{\prime} \rightarrow \theta\right)}{\pi(\theta) q\left(\theta \rightarrow \theta^{\prime}\right)} \leq 1
$$

Para que $f(\theta \mid \mathcal{D})$ seja a distribuição estacionária, devemos mostrar que $f(\theta \mid \mathcal{D}) r\left(\theta \rightarrow \theta^{\prime}\right)=$ $f\left(\theta^{\prime} \mid \mathcal{D}\right) r\left(\theta^{\prime} \rightarrow \theta\right)$. Logo,

$$
\begin{aligned}
f(\theta \mid \mathcal{D}) r\left(\theta \rightarrow \theta^{\prime}\right) & =f(\theta \mid \mathcal{D}) q\left(\theta \rightarrow \theta^{\prime}\right) \mathbb{P}\left(\mathcal{D} \mid \theta^{\prime}\right) h\left(\theta, \theta^{\prime}\right) \\
& =\frac{\mathbb{P}(\mathcal{D} \mid \theta) \pi(\theta)}{\mathbb{P}(\mathcal{D})}\left\{q\left(\theta \rightarrow \theta^{\prime}\right) \mathbb{P}\left(\mathcal{D} \mid \theta^{\prime}\right) \frac{\pi\left(\theta^{\prime}\right) q\left(\theta^{\prime} \rightarrow \theta\right)}{\pi(\theta) q\left(\theta \rightarrow \theta^{\prime}\right)}\right\} \\
& =\frac{\mathbb{P}\left(\mathcal{D} \mid \theta^{\prime}\right) \pi\left(\theta^{\prime}\right)}{\mathbb{P}(\mathcal{D})}\left\{q\left(\theta^{\prime} \rightarrow \theta\right) \mathbb{P}(\mathcal{D} \mid \theta) \frac{\pi(\theta) q\left(\theta \rightarrow \theta^{\prime}\right)}{\pi(\theta) q\left(\theta \rightarrow \theta^{\prime}\right)}\right\} \\
& =f\left(\theta^{\prime} \mid \mathcal{D}\right) q\left(\theta^{\prime} \rightarrow \theta\right) \mathbb{P}(\mathcal{D} \mid \theta) \\
& \stackrel{(I)}{=} f\left(\theta^{\prime} \mid \mathcal{D}\right) q\left(\theta^{\prime} \rightarrow \theta\right) \mathbb{P}(\mathcal{D} \mid \theta) h\left(\theta^{\prime}, \theta\right) \\
& =f\left(\theta^{\prime} \mid \mathcal{D}\right) r\left(\theta^{\prime} \rightarrow \theta\right)
\end{aligned}
$$

Na passagem $(I)$ usamos o fato de que como vale (4.2), então $h\left(\theta^{\prime}, \theta\right)=1$. A demonstração para $\frac{\pi\left(\theta^{\prime}\right) q\left(\theta^{\prime} \rightarrow \theta\right)}{\pi(\theta) q\left(\theta \rightarrow \theta^{\prime}\right)}>1$ é análoga.

Note que o algoritmo E se restringe aos espaços amostrais discretos. Assim, como feito anteriormente, dado uma medida de discrepância adequada $d$, uma precisão $\varepsilon>0$ e um conjunto de $p$ estatísticas $T=\left(T_{1}, \ldots, T_{p}\right)$, a etapa $\mathrm{E} 3$ do algoritmo pode ser alterada de duas formas:

E3': se $d\left(\mathcal{D}^{\prime}, \mathcal{D}\right) \leq \varepsilon$, vá para E4, caso contrário permaneça no estado $\theta$ e vá para E1.

E3": se $d\left(T^{\prime}, T\right) \leq \varepsilon$, vá para E4, caso contrário permaneça no estado $\theta$ e vá para E1.

Considerando E3' ao invés de E3, a distribuição estacionária é $f\left(\theta \mid d\left(\mathcal{D}^{\prime}, \mathcal{D}\right) \leq \varepsilon\right)$, enquanto que ao considerar E3", teremos $f\left(\theta \mid d\left(T^{\prime}, T\right) \leq \varepsilon\right)$ como distribuição estacionária.

Exemplo 2 - Distribuições $\alpha$-estáveis (revisitado - parte 1). Aplicamos o algoritimo E usando o passo E3" aos mesmos dados do Exemplo 1, cujo valor nominal do parâmetro de interesse $\delta$ é igual a 2. O kernel de transição escolhido é o passeio aleatório com desvio-padrão 0.1, e a precisão do algoritmo é dada por $\varepsilon=0.1$. Consideramos a mesma priori para $\delta$, isto é, $\delta \sim N(0,25)$, média amostral como valor inicial do algoritmo e mediana amostral como estatística resumo. Realizamos 10000 repetições do algoritmo descartando as 5000 primeiras observações simuladas. A estimativa da média aproximada a posteriori ficou igual a 1.897 e o desvio-padrão da amostra obtida foi 0.345 , lembrando que o resultado obtido pelo algoritmo D foi 1.842 para a média e 0.432 para o desviopadrão, ou seja, os resultados são praticamente os mesmos. A Figura 4.5 ilustra o aprendizado sobre $\delta$ através do histograma da amostra obtida pelo algoritmo E e a curva da densidade a priori. Na mesma figura mostramos o traço dos valores aceitos indicando um bom mixing da cadeia.

Realizamos novamente 100 simulações de Monte Carlo considerando duas prioris diferentes para $\delta$, sendo elas $N(0,25)$ e $N(-2,1)$, lembrando que esta última não produz bons resultados através do algoritmo D. A Tabela 4.4 nos fornece a média e o desvio-padrão das estimativas obtidas nas simulações de Monte Carlo segundo cada priori. Note que mesmo no caso onde há conflito de informação, isto é, quando $\delta \sim N(-2,1)$ a priori, o algoritmo E produz resultados satisfatórios onde o algoritmo D falha. Esta característica do algoritmo E torna seu uso atrativo em situações onde utiliza-se prioris pouco informativas ou quando o valor verdadeiro do parâmetro encontra-se na cauda da distribuição a priori. O caso onde a priori é $N(0,25)$ retorna resultados similares aos obtidos pelo algoritmo D. 
Valores aceitos

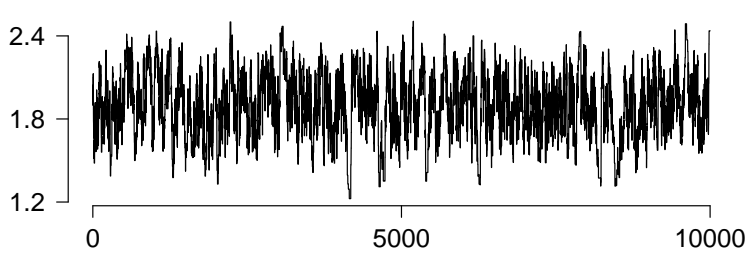

Posteriori aproximada

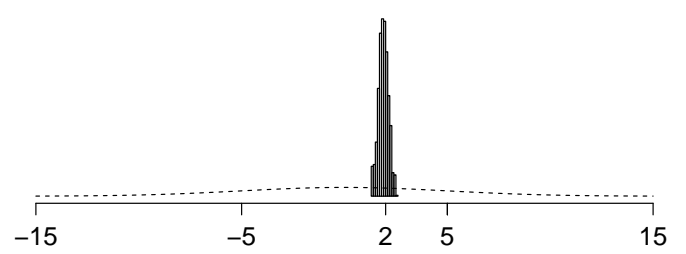

Figura 4.5: Traço dos valores de $\delta$ obtidos pelo algoritmo E para uma amostra de tamanho 100 da distribuição $S(1.5,0.3,1, \delta=2)$ e histograma desses valores após exclusão dos 5000 primeiros. A curva tracejada representa a função de densidade a priori que neste caso é $N(0,25)$.

\begin{tabular}{cc}
\hline \hline Priori & média (desvio-padrão) \\
\hline$N(0,25)$ & $1.9932(0.1788)$ \\
$N(-2,1)$ & $1.8068(0.2705)$ \\
\hline \hline
\end{tabular}

Tabela 4.4: Média e devio-padrão das estimativas (média a posteriori) obtidas para $\delta$ usando o algoritmo E em 100 simulações de Monte Carlo utilizando as prioris $N(0,25)$ e $N(-2,1)$ para $\delta$.

Exemplo 3 - Processo de médias móveis. Este exemplo foi apresentado em Marin et al. (2012) e ilustra uma aplicação do método $\mathrm{ABC}$ na área de inferência em processos estocásticos.

O processo de médias móveis de ordem $q$, denotado por $M A(q)$, é um processo estocástico $\left\{Y_{k}, k \in \mathbb{N}^{*}\right\}$ definido por

$$
Y_{k}=Z_{k}+\sum_{i=1}^{q} \theta_{i} Z_{k-i},
$$

onde $\left(Z_{k}\right)_{k \in \mathbb{Z}}$ é uma sequência i.i.d de variáveis $N(0,1)$, e uma condição padrão de identificabilidade é que as raízes do polinômio

$$
Q(x)=1-\sum_{i=1}^{q} \theta_{i} x^{i},
$$

devem estar fora do círculo unitário.

Considere o processo $M A(2)$. Neste caso $\theta=\left(\theta_{1}, \theta_{2}\right)$ deve satisfazer

$$
\left\{\begin{array}{c}
-2<\theta_{1}<2 \\
\theta_{1}+\theta_{2}>-1 \\
\theta_{1}-\theta_{2}<1
\end{array}\right.
$$

Suponha que foi observado uma série $y=\left(y_{1}, \ldots, y_{n}\right)^{T}$ com $n=100$ de um processo $M A(2)$ com parâmetros $\theta=\left(\theta_{1}, \theta_{2}\right)=(0.6,0.2)$. Assuma que $\theta$ seja desconhecido e que os algoritmos $\mathrm{C}$ e D serão utilizados para obter uma amostra aproximada da posteriori de $\theta$. Note que neste caso a 
função de verossimilhança é conhecida e dada por

$$
f(y \mid \theta)=(2 \pi)^{-n / 2}\left|\sum\right|^{-1 / 2} \exp \left\{-\frac{1}{2} y^{T} \sum^{-1} y\right\}
$$

onde $\sum=\sum_{n \times n}$ é a matriz de covariância correspondente ao modelo $M A(2)$ (Hamilton, 1994).

Assuma a priori que $\theta$ seja distribuído uniformemente no triângulo determinado pelas relações (4.3). Deste modo, a distribuição a posteriori para $\theta$ será a própria verossimilhança (4.4) limitada aos valores que $\theta_{1}$ e $\theta_{2}$ podem assumir. A Figura 4.6 nos apresenta essa posteriori que foi obtida numericamente. Para o algoritmo D considere como estatísticas resumo as duas primeiras autocovariâncias amostrais definidas por

$$
\tau_{j}=\sum_{k=j+1}^{n} y_{k} y_{k-j}, \quad j=1,2,
$$

e assuma também a distância Euclidiana como métrica nos algoritmos.

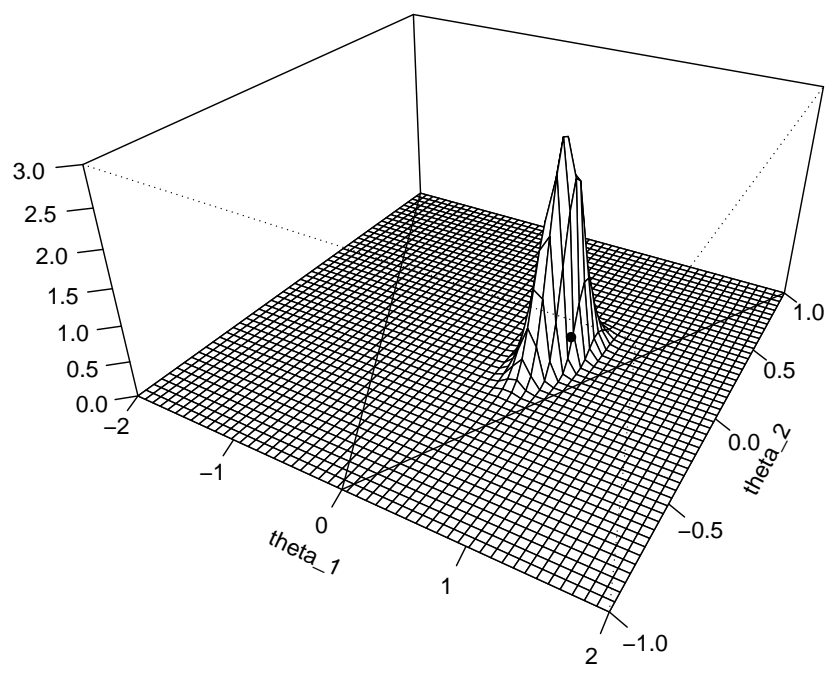

Figura 4.6: Distribuição a posteriori para $\theta=\left(\theta_{1}, \theta_{2}\right)$. O triângulo representa os valores que os parâmetros podem assumir.

Foram realizadas $10^{6}$ repetições dos algoritmos $\mathrm{C}$ e $\mathrm{D}$ tomando três valores distintos para $\varepsilon$, escolhidos de modo a aceitar $10 \%, 1 \%$ e $0.1 \%$ das simulações. A Figura 4.7 apresenta os gráficos de dispersão das amostras obtidas pelo algoritmo C, enquanto a Figura 4.8 apresenta as amostras obtidas pelo algoritmo D. Cada gráfico apresenta curvas de nível da distribuição a posteriori teórica, bem como os limites da distribuição a priori considerada. Estas figuras ilustram o efeito da escolha de estatísticas resumo representativas para o parâmetro de interesse, de fato, as duas primeiras autocovariâncias amostrais são essenciais na determinação da ordem do modelo $M A(2)$. Observe que no caso onde foi utilizado o algoritmo $\mathrm{C}$, a amostra obtida tende a se concentrar no meio do triângulo definido pelas relações (4.3), não importando o quão pequeno $\varepsilon$ seja tomado. Este é um exemplo que nos mostra que tomar a distância Euclidiana entre duas séries temporais torna o $\mathrm{ABC}$ pouco preciso. O mesmo não acontece quando o algoritmo $\mathrm{D}$ é utilizado pois, neste caso, as amostras obtidas concentram-se cada vez mais em torno da posteriori verdadeira de $\theta$ quando $\varepsilon$ diminui. A média a posteriori aproximada para os parâmetros $\theta_{1}$ e $\theta_{2}$ usando o algoritmo $\mathrm{D}$ foram 
respectivamente 0.550 e 0.164 com desvios padrão dados por 0.136 e 0.185 respectivamente. Os gráficos da Figura 4.9 apresentam as posterioris marginais aproximadas de $\theta$ obtidas pelo algoritmo $\mathrm{D}$, juntamente com as curvas das marginais teóricas que foram obtidas através de integração numérica. Nesta figura também é possível notar a melhoria na precisão conforme o valor de $\varepsilon$ diminui.
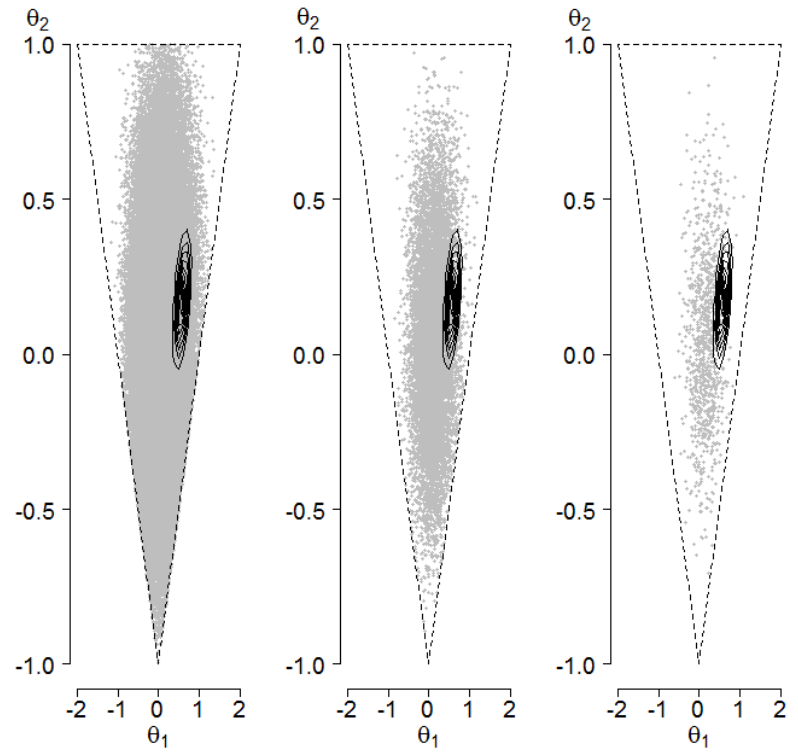

Figura 4.7: Dispersão dos pontos aceitos pelo $A B C$ (algoritmo $C$ ) variando os valores de $\varepsilon$ de modo a aceitar $10 \%$ (esquerda), $1 \%$ (centro) e $0.1 \%$ (direita) das simulações. Cada gráfico possui curvas de nivel da distribuição a posteriori teórica e os triângulos tracejados representam a distribuição a priori.
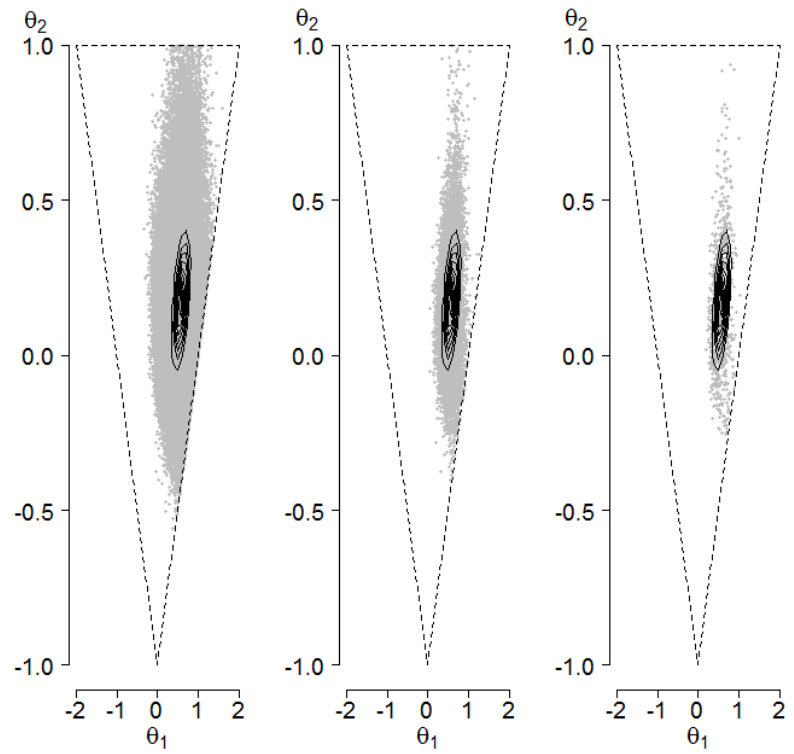

Figura 4.8: Dispersão dos pontos aceitos pelo $A B C$ (algoritmo D) utilizando as duas primeiras autocovariâncias como estatísticas resumo. Os valores de $\varepsilon$ foram tomados de modo a aceitar $10 \%$ (esquerda), $1 \%$ (centro) e $0.1 \%$ (direita) das simulações. Cada gráfico possui curvas de nível da distribuição a posteriori teórica e os triângulos tracejados representam a distribuição a priori. 

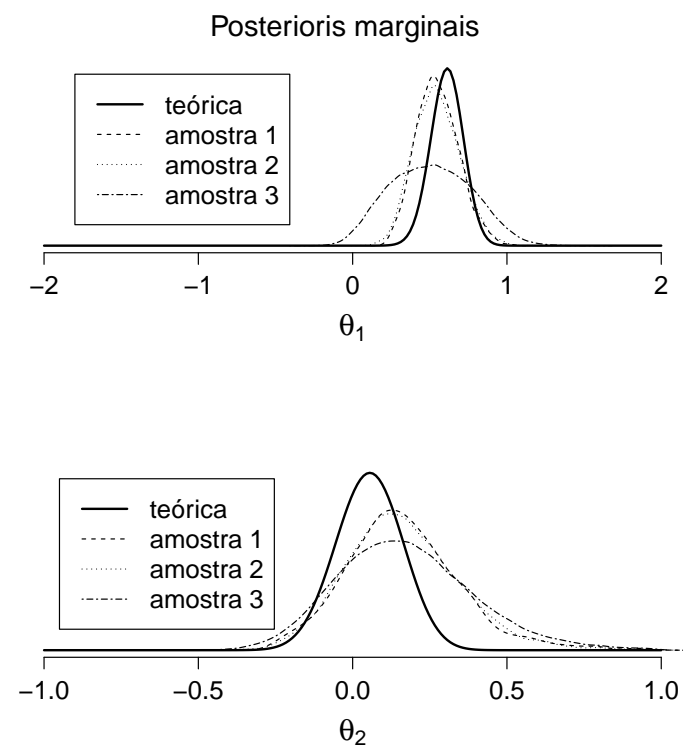

Figura 4.9: Distribuições a posteriori marginais de $\theta=\left(\theta_{1}, \theta_{2}\right)$ obtidas pelo algoritmo D. A curva sólida representa a distribuição teórica, enquanto as curvas obtidas através das amostras 1, 2 e 3 correspondem aos valores de $\varepsilon$ considerados no algoritmo.

\subsection{Sobre a escolha de estatísticas resumo}

\subsubsection{Critério de mínima entropia}

Como mencionado anteriormente, a escolha de estatísticas resumo para uso em métodos $\mathrm{ABC}$ corresponde a uma etapa delicada do processo de implementação do método, uma vez que a perda de informação induzida pelo uso de estatísticas insuficientes pode tornar o método pouco preciso. Como na prática são raros os casos onde podemos utilizar estatísticas suficientes, devemos aos menos procurar estatísticas que sejam representativas, isto é, aquelas que produzam uma perda de informação mínima com respeito ao parâmetro de interesse. Em princípio obtemos informação máxima usando todas as estatísticas resumo disponíveis, já que na pior das hipóteses certas estatísticas não adicionarão informação alguma sobre $\theta$, e incluir tais estatísticas não afeta a posteriori para $\theta$. No entanto, na prática, a adição de uma nova estatística àquelas consideradas num método de rejeição como o algoritmo D resultará em um número menor de iterações sendo aceitas, resultando em uma adição de ruído ao estimador empírico baseado no conjunto de $\theta$ 's aceitos. Logo, uma estratégia de escolha de estatísticas resumo deve levar em conta se a inclusão de uma nova estatística melhora ou não a aproximação da posteriori resultante.

Uma alternativa simples é motivada pela definição de suficiência no contexto da Teoria da Informação (Cover e Thomas, 2006), embora essa só seja útil após uma análise prévia das estatísticas a serem utilizadas, assim como foi feito no Exemplo 1 (Figura 4.1).

Definição 4.1 (Entropia). A entropia $\mathbf{h}(X)$ de uma variável aleatória $X$ com densidade $f(x)$ é definida como

$$
\mathbf{h}(X)=-\int f(x) \log f(x) d x,
$$

onde a integral, caso exista, é definida no suporte de $f$.

Definição 4.2 (Informação mútua). Considere duas variáveis aleatórias $X$ e $Y$ com densidade conjunta $f(x, y)$ e densidades marginais $f(x)$ e $g(y)$ respectivamente. A informação mútua entre $X$ e $Y$, denotada por $I(X ; Y)$, é definida por 


$$
I(X ; Y)=\iint f(x, y) \log \frac{f(x, y)}{f(x) g(y)} d x d y,
$$

onde a integral, caso exista, é definida nos valores que $X$ e $Y$ podem assumir.

Definição 4.3. A função $T(X)$ é uma estatística suficiente para a família $\{f(x \mid \theta) ; \theta \in \Theta\}$ se, $e$ somente se, $I(\theta ; T(X))=I(\theta ; X)$

Em geral, se $T(X)$ é insuficiente, temos que $I(\theta ; T(X)) \leq I(\theta ; X)$. Logo, a Definição 4.3 sugere que quanto mais próximo $I(\theta ; T(X))$ está de $I(\theta ; X)$, menor será a perda de informação induzida por $T$ com respeito a $\theta$. Um resultado clássico da teoria da informação estabelece que

$$
I(\theta ; T(X))=\mathbf{h}(\theta)-\mathbf{h}(\theta \mid T(X)) .
$$

Ou seja, a partir da inequação $I(\theta ; T(X))<I(\theta ; X)$ e de (4.5), segue que maximizar a informação mútua $I(\theta ; T(X))$ é equivalente a minimizar a entropia condicional $\mathbf{h}(\theta \mid T(X))$ que é exatamente a entropia da distribuição a posteriori obtida pelos métodos $\mathrm{ABC}$ que utilizam estatísticas resumo, e quando $\varepsilon \rightarrow 0$.

Portanto, este é um critério que pode ser utilizado para selecionar uma (ou mais) estatística(s) resumo ótima(s) de um conjunto de estatísticas resumo disponível, estimando-se a entropia $\mathbf{h}(\theta \mid T(X))$ com base na amostra obtida pelo ABC. É importante ressaltar que essa escolha é ótima com respeito às estatísticas consideradas na implementação do método, isto é, se todas as estatísticas consideradas tiverem um mau desempenho no método ABC utilizado, estaremos apenas escolhendo aquela(s) que nos faz errar menos. Logo, este critério é recomendado apenas quando se está na dúvida sobre qual estatística está produzindo resultados melhores ou se a inclusão de novas estatísticas melhoram ou pioram a análise, como observado anteriormente. A entropia da distribuição a posteriori pode ser estimada a partir do método apresentado em Singh et al. (2003).

Exemplo 4 - Distribuições $\alpha$-estáveis (revisitado - parte 2). No Exemplo 1 consideramos 4 conjuntos de estatísticas resumo para serem utilizados no algoritmo D, a saber, média amostral, mediana amostral, percentis empíricos e todas as estatísticas anteriores conjuntamente. Embora as quatro abordagens sejam capazes de produzir resultados satisfatórios, concluímos através da Figura 4.3 que a média amostral produzia os piores resultados, enquanto os melhores eram obtidos quando se utilizava a mediana amostral como estatística resumo. Repetindo as simulações nas mesmas condições do Exemplo 1, utilizamos o critério de mínima entropia e contamos quantas vezes cada conjunto de estatísticas resumo foi selecionado. De 100 simulações, a média amostral não foi selecionada, a mediana amostral foi selecionada em $75 \%$ das vezes, os percentis em $15 \%$ e o conjunto com todas as estatísticas anteriores foi selecionado em $10 \%$ das simulações, o que está de acordo com o que foi observado no Exemplo 1.

\subsubsection{Critério de $\epsilon$-suficiência}

Assim como na proposta de mínima entropia, Joyce e Marjoram (2008) propõem uma abordagem sequencial para a escolha de estatísticas resumo. Suponha que $T=\left\{T_{1}, \ldots, T_{p-1}\right\}$ seja um conjunto de estatísticas resumo e que $T_{p}$ seja um candidato a entrar neste conjunto. Joyce e Marjoram (2008) procuram responder se a adição de $T_{p}$ ao conjunto $T$ melhora a qualidade da inferência, ou se a informação sobre o parâmetro de interesse induzida por $T_{p}$ é tão pequena que esta pode ser ignorada. A idéia parte do princípio de $\epsilon$-suficiência a ser definida a seguir.

Definição 4.4 ( $\epsilon$-suficiência). As estatísticas $T_{1}, \ldots, T_{p-1}$ são ditas $\epsilon$-suficientes com respeito a uma estatística $X$ se

$$
\sup _{\theta} \ln \mathbb{P}\left(X \mid T_{1}, \ldots, T_{p-1}, \theta\right)-\inf _{\theta} \ln \mathbb{P}\left(X \mid T_{1}, \ldots, T_{p-1}, \theta\right) \leq \epsilon
$$


Se $X$ representa todo o conjunto de dados e $\epsilon=0$, então a definição 4.4 se reduz à definição de suficiência. O objetivo é tomar $X=T_{p}$.

Definição 4.5. O escore de $T_{p}$ relativo a $T_{1}, \ldots, T_{p-1}$ é definido por

$$
\delta_{p}=\sup _{\theta} \ln \mathbb{P}\left(T_{p} \mid T_{1}, \ldots, T_{p-1}, \theta\right)-\inf _{\theta} \ln \mathbb{P}\left(T_{p} \mid T_{1}, \ldots, T_{p-1}, \theta\right)
$$

ou,

$$
e^{\delta_{p}}=\frac{\sup _{\theta} \mathbb{P}\left(T_{p} \mid T_{1}, \ldots, T_{p-1}, \theta\right)}{\inf _{\theta} \mathbb{P}\left(T_{p} \mid T_{1}, \ldots, T_{p-1}, \theta\right)} .
$$

Quando o escore $\delta_{p}$ estiver abaixo de um certo limiar a adição de novas estatísticas não melhora a inferência sobre $\theta$. Se $\pi(\theta)$ é uma priori para $\theta$, segue que

$$
\begin{aligned}
f\left(\theta \mid T_{1}, \ldots, T_{p}\right) & =\frac{\mathbb{P}\left(T_{1}, \ldots, T_{p-1} \mid \theta\right) \mathbb{P}\left(T_{p} \mid T_{1}, \ldots, T_{p-1}, \theta\right) \pi(\theta)}{\int \mathbb{P}\left(T_{1}, \ldots, T_{p-1} \mid \theta\right) \mathbb{P}\left(T_{p} \mid T_{1}, \ldots, T_{p-1}, \theta\right) \pi(\theta) d \theta} \\
& \leq \frac{\mathbb{P}\left(T_{1}, \ldots, T_{p-1} \mid \theta\right) \pi(\theta)}{\int \mathbb{P}\left(T_{1}, \ldots, T_{p-1} \mid \theta\right) \pi(\theta) d \theta} \frac{\sup _{\theta} \mathbb{P}\left(T_{p} \mid T_{1}, \ldots, T_{p-1}, \theta\right)}{\inf _{\theta} \mathbb{P}\left(T_{p} \mid T_{1}, \ldots, T_{p-1}, \theta\right)} \\
& =f\left(\theta \mid T_{1}, \ldots, T_{p-1}\right) e^{\delta_{p}}
\end{aligned}
$$

ou seja,

$$
R_{p}(\theta):=\frac{f\left(\theta \mid T_{1}, \ldots, T_{p}\right)}{f\left(\theta \mid T_{1}, \ldots, T_{p-1}\right)} \leq e^{\delta_{p}}
$$

e usando o mesmo raciocínio, segue que $R_{p}(\theta) \geq e^{-\delta_{p}}$.

Esta razão de chances $R_{p}(\theta)$ mostra como o escore da estatística resumo proposta se relaciona com a distribuição a posteriori de interesse, sendo que as posterioris $f\left(\theta \mid T_{1}, \ldots, T_{p}\right)$ e $f\left(\theta \mid T_{1}, \ldots, T_{p-1}\right)$ podem ser estimadas via ABC. Note que escolhendo um escore limiar suficientemente pequeno, teremos $e^{\delta_{p}} \approx e^{-\delta_{p}} \approx 1$ e a diferença entre as posterioris $f\left(\theta \mid T_{1}, \ldots, T_{p}\right)$ e $f\left(\theta \mid T_{1}, \ldots, T_{p-1}\right)$ será pequena. Além disso, temos que $e^{\delta_{p}}$ pode ser obtido a partir de $R_{p}(\theta)$, pois

$$
\begin{aligned}
\mathbb{P}\left(T_{p} \mid T_{1}, \ldots, T_{p-1}, \theta\right) & =\frac{f\left(\theta, T_{p} \mid T_{1}, \ldots, T_{p-1}\right)}{f\left(\theta \mid T_{1}, \ldots, T_{p-1}\right)} \\
& =\frac{f\left(\theta \mid T_{1}, \ldots, T_{p}\right)}{f\left(\theta \mid T_{1}, \ldots, T_{p-1}\right)} \mathbb{P}\left(T_{p} \mid T_{1}, \ldots, T_{p-1}\right) \\
& =R_{p}(\theta) \mathbb{P}\left(T_{p} \mid T_{1}, \ldots, T_{p-1}\right),
\end{aligned}
$$

e assim,

$$
e^{\delta_{p}}=\frac{\sup _{\theta} \mathbb{P}\left(T_{p} \mid T_{1}, \ldots, T_{p-1}, \theta\right)}{\inf _{\theta} \mathbb{P}\left(T_{p} \mid T_{1}, \ldots, T_{p-1}, \theta\right)}=\frac{\sup _{\theta} R_{p}(\theta)}{\inf _{\theta} R_{p}(\theta)} .
$$

Joyce e Marjoram (2008) implementaram esse método da seguinte maneira: Sendo $\mathcal{D}$ o conjunto de dados com $t_{i}=T_{i}(\mathcal{D})(i=1, \ldots, p)$, podemos obter uma aproximação para $f\left(\theta \mid t_{1}, \ldots, t_{p}\right)$ usando algum método ABC como o algoritmo D. Essa aproximação pode ser usada para aproximar a posteriori de $\theta$ dado qualquer subconjunto de $\left\{t_{1}, \ldots, t_{p}\right\}$ sendo possível obter uma aproximação para $R_{p}(\theta)$ denotada por $R_{p}^{*}(\theta)$. Considere agora a discretização de $\theta$ no conjunto $\left\{\theta_{1}, \ldots, \theta_{L}\right\}$. Seja $N_{p}$ o número de observações aceitas no método de rejeição usando as estatísticas $T_{1}, \ldots, T_{p}$ e $N_{p-1}(i)$ o número de observações aceitas quando $\theta=\theta_{i}$ considerando as estatísticas $T_{1}, \ldots, T_{p-1}$. Sob a hipótese nula de que a estatística $T_{p}$ não adiciona informação para a posteriori de $\theta$, o número esperado de valores aceitos depois de adicionar a $p$-ésima estatística é $\frac{N_{p-1}(i) N_{p}}{N_{p-1}}=: n(i)$. Com isso, podemos calcular o desvio-padrão amostral de $n(i)(i=1, \ldots, L)$ permitindo que $R_{p}^{*}(\theta)$ seja diferente de 1 em até 4 vezes esse desvio-padrão. 
Vale observar que a ordem das estatísticas a serem incluídas influencia o mecanismo de escolha do método, e não há como estabelecer a priori quais estatísticas carregam mais informação sobre o parâmetro de interesse. O ideal seria ir adicionando estatísticas em ordem decrescente, isto é, da que contém mais informação para a que contém menos, mas tal ordenação é praticamente impossível de se obter na prática. Um alternativa seria usar o ABC para obter amostras a posteriori segundo cada estatística e usar o critério de mínima entropia para determinar uma ordem para essas estatísticas. Outra abordagem seria considerar todos os subconjuntos de estatísticas, mas em casos onde o número de estatísticas é grande, o método torna-se computacionalmente intratável. A alternativa proposta por Joyce e Marjoram (2008) consiste em adicionar uma estatística escolhida aleatoriamente em cada passo, e se esta for aceita no conjunto atual, novos testes devem determinar se as demais devem permanecer ou não no conjunto.

\subsubsection{ABC semiautomático}

Fearnhead e Prangle (2012) enfraquecem o requerimento de que a posteriori obtida pelo ABC seja uma boa aproximação para $f(\theta \mid \mathcal{D})$, argumentando que a mesma seja uma boa aproximação apenas em termos da precisão de certos estimadores. Além disso, consideram que seu método possui uma importante propriedade chamada calibração. Neste contexto, um método ABC é dito calibrado se as afirmações probabilísticas que são feitas sobre a posteriori obtida dele são apropriadas e, em particular, essa posteriori pode ser utilizada para quantificar incerteza nas estimativas. Deste modo, os autores argumentam que usando tal critério é possível construir posterioris através de métodos ABC que possuem boas propriedades inferenciais. A abordagem de Fearnhead e Prangle (2012) é chamada de $\mathrm{ABC}$ semiautomático, pois esta seleciona estatísticas resumo automaticamente, mas algumas características do método deve ser escolhidas de maneira ad-hoc.

Seja $\mathcal{D}$ um conjunto de dados e $\theta^{T}=\left(\theta_{1}, \ldots, \theta_{q}\right)$ parâmetros do modelo. Tal método seleciona estatísticas que minimizam a perda quadrática

$$
L\left(\theta_{V}, \hat{\theta}\right)=\left(\theta_{V}-\hat{\theta}\right)^{T} A\left(\theta_{V}-\hat{\theta}\right),
$$

onde $\theta_{V}$ é o verdadeiro valor de $\theta, \hat{\theta}$ uma estimativa e $A$ é uma matriz $p \times p$ positiva definida.

Tal perda é minimizada por $E(\theta \mid \mathcal{D})$ e assim, os autores demonstram que as estatísticas resumo ótimas são iguais as médias a posteriori de cada um dos parâmetros.

Como a esperança $E(\theta \mid \mathcal{D})$ é desconhecida, Fearnhead e Prangle (2012) propõem estimar essa esperança através de modelos de regressão como segue: Em primeiro lugar é considerada uma função $h(\cdot)$, de modo que $h(\mathcal{D})$ seja um vetor de transformações, possivelmente não lineares, dos dados. A escolha mais simples para $h$ é a função identidade mas, na prática, incluir outras transformações pode ser benéfico. Além disso, ao invés de aplicar a função $h$ nos dados, esta pode ser aplicada às estatísticas potencialmente informativas sobre o parâmetro de interesse (Blum et al., 2013).

Os $M$ valores simulados $\theta_{i}^{(1)}, \ldots, \theta_{i}^{(M)}$ do $i$-ésimo parâmetro $(i=1, \ldots, q)$ são utilizados como respostas, enquanto as transformações dos dados simulados $h\left(\mathcal{D}^{(1)}\right), \ldots, h\left(\mathcal{D}^{(M)}\right)$ são utilizadas como variáveis explicativas para ajustar o modelo

$$
\theta_{i}=E\left(\theta_{i} \mid \mathcal{D}\right)+\eta_{i}=\beta_{0}^{(i)}+\beta^{(i)} h(\mathcal{D})+\eta_{i}
$$

onde $\eta_{i}$ é um ruído com média nula.

A função ajustada por mínimos quadrados $\hat{\beta}_{0}^{(i)}+\hat{\beta}^{(i)} h(\mathcal{D})$ é então uma estimativa para $E\left(\theta_{i} \mid \mathcal{D}\right)$. O termo constante pode ser ignorado na prática, já que o $\mathrm{ABC}$ utiliza apenas a diferença entre estatísticas resumo (cancelando os $\hat{\beta}_{0}^{(i)}$ s) e assim, a $i$-ésima estatística resumo a ser utilizada no método $\mathrm{ABC}$ é $\hat{\beta}^{(i)} h(\mathcal{D})$.

Deste modo, o método $\mathrm{ABC}$ semiautomático deve ser executado da seguinte maneira:

(SA1) (Opcional) Aplique um método ABC piloto para determinar uma região com alta probabilidade a posteriori, 
(SA2) simule valores para os parâmetros da priori restrita à região obtida no passo (SA1) e simule dados do modelo com esses valores de parâmetros,

(SA3) use os parâmetros e dados simulados no passo (SA2) para estimar as estatísticas resumo,

(SA4) aplique um método $\mathrm{ABC}$ com as estatísticas resumo obtidas no passo (SA3).

O passo (SA1) é opcional e serve para tornar o método mais eficiente quando se utiliza prioris não informativas, especialmente se forem impróprias. Caso a priori utilizada seja razoavelmente informativa, este passo pode ser ignorado. Note que a repetição do passo (SA3) com diferentes escolhas para $h(\cdot)$ pode ser feita sem a necessidade de simular novos parâmetros e dados e com isso, procedimentos de comparação de modelos usuais, como o critério de informação Bayesiano (BIC), podem ser utilizados para escolher estatísticas resumo que são obtidas através das regressões lineares utilizando diferentes variáveis explicativas.

\subsection{Pós-processamento da amostra ABC}

No Exemplo 3 ficou evidente como a escolha de $\varepsilon$ influencia na precisão do método $\mathrm{ABC}$, especialmente quando $\varepsilon$ foi escolhido de modo a aceitar $10 \%$ das simulações. Beaumont et al. (2002) propõem um pós-processamento da amostra obtida pelo ABC, melhorando a aproximação, especialmente em casos onde $\varepsilon$ não pode ser tomado muito pequeno. Dado um conjunto de dados $\mathcal{D}$, e um conjunto de estatísticas resumo $T=\left(T_{1}, \ldots, T_{p}\right)$, este pós-processamento busca modelar a discrepância entre $T(\mathcal{D})$ e $T\left(\mathcal{D}_{i}\right)$, onde $\mathcal{D}_{i}$ é um conjunto de dados simulados do modelo com parâmetro $\theta_{i}$.

Suponha por simplicidade que o parâmetro $\theta$ do modelo é univariado. O caso multivariado é feito aplicando-se o método para cada parâmetro individualmente. Temos que a esperança condicional $m(T(\mathcal{D}))=E[\theta \mid T(\mathcal{D})]$ pode ser usada para estimar $\theta$, mas a perda de informação sobre $\theta$ induzida pela estatística $T$ produz um aumento no erro de estimação.

Assim, podemos considerar a seguinte regressão na região de $T(\mathcal{D})$ :

$$
\theta^{i}=m\left(T\left(\mathcal{D}_{i}\right)\right)+\omega^{i},
$$

onde os $\theta^{i},(i=1, \ldots, N)$ são os $N$ valores aceitos pelo método $\mathrm{ABC}$ utilizado, $T\left(\mathcal{D}_{i}\right)$ é o valor da estatística resumo aplicada aos dados simulados do modelo com parâmetro $\theta^{i}$ e $\omega^{i}$ é um ruído com média nula.

Para estimar a média condicional $m(\cdot)$, Beaumont et al. (2002) assumem o modelo linear

$$
m\left(T\left(\mathcal{D}_{i}\right)\right)=\beta_{0}+\beta_{1}^{\prime} T\left(\mathcal{D}_{i}\right),
$$

na vizinhança de $T(\mathcal{D})$ e uma estimativa dessa média, $\hat{m}(\cdot)$, é obtida utilizando o método de mínimos quadrados ponderados, ou seja, minimizando

$$
\sum_{i=1}^{N} \eta^{i}\left\|m\left(T\left(\mathcal{D}_{i}\right)\right)-\theta^{i}\right\|^{2},
$$

onde $\eta^{i}=K_{\lambda}\left(\left\|T\left(\mathcal{D}_{i}\right)-T(\mathcal{D})\right\|\right)$ é o kernel de Epanechnikov dado por,

$$
K_{\lambda}(x)= \begin{cases}c \lambda^{-1}\left[1-(x / \lambda)^{2}\right], & x \leq \lambda \\ 0, & x>\lambda,\end{cases}
$$

onde $c$ é uma constante normalizadora.

O uso de regressão linear local na vizinhança de $T(\mathcal{D})$ é utilizada pois a suposição de que a regressão seja linear globalmente é implausível. Logo, a amostra a posteriori ajustada pelo método de pós-processamento de Beaumont et al. (2002) é dada por 


$$
\theta^{* i}=\hat{m}(T(\mathcal{D}))+\left[\theta^{i}-\hat{m}\left(T\left(\mathcal{D}_{i}\right)\right)\right], \quad(i=1, \ldots, N) .
$$

Note que a aproximação para a média a posteriori de $\theta$ obtida através desse método é dada por

$$
\frac{\sum_{i} \theta^{* i} K_{\lambda}\left(\left\|T\left(\mathcal{D}_{i}\right)-T(\mathcal{D})\right\|\right)}{\sum_{i} K_{\lambda}\left(\left\|T\left(\mathcal{D}_{i}\right)-T(\mathcal{D})\right\|\right)}
$$

e se o kernel escolhido for uniforme, isto é, $K_{\lambda}(x)=\mathbb{I}_{\{x \leq \lambda\}}(x)$, então a média a posteriori é estimada por

$$
\frac{\sum_{i} \theta^{i} \mathbb{I}_{\left\{\left\|T\left(\mathcal{D}_{i}\right)-T(\mathcal{D})\right\| \leq \lambda\right\}}\left(\left\|T\left(\mathcal{D}_{i}\right)-T(\mathcal{D})\right\|\right)}{\sum_{i} \mathbb{I}_{\left\{\left\|T\left(\mathcal{D}_{i}\right)-T(\mathcal{D})\right\| \leq \lambda\right\}}\left(\left\|T\left(\mathcal{D}_{i}\right)-T(\mathcal{D})\right\|\right)}
$$

que é simplesmente a estimativa da média a posteriori obtida pelo algoritmo $\mathrm{D}$, ou seja, o algoritmo $\mathrm{D}$ pode ser visto como um caso particular do método de regressão linear local de Beaumont et al. (2002).

Outras técnicas de pós processamento da amostra do ABC podem ser obtidas em Blum e François (2010), onde a regressão linear local proposta no método anterior é substituída por uma regressão não linear com heterocedasticidade, e em Leuenberger et al. (2010) onde se utiliza regressão inversa.

O método de pós-processamento de Beaumont et al. (2002) é similar às ideias do ABC semiautomático de Fearnhead e Prangle (2012), apresentado na subseção 4.1.3. Enquanto o último usa regressão linear para construir estatísticas resumo e é aplicado a todos os parâmetros simulados e suas respectivas estatísticas calculadas nas amostras simuladas, o outro procura reduzir o erro entre $f(\theta \mid T=t)$ e a aproximação obtida pelo $\mathrm{ABC}$, sendo aplicado apenas aos parâmetros aceitos pelo $\mathrm{ABC}$ e suas respectivas estatísticas calculadas nas amostras simuladas. Além disso, o método de pós-processamento assume uma escolha prévia de estatísticas resumo potencialmente informativas.

Exemplo 6 - Processo de médias móveis (revisitado - parte 1). No Exemplo 3 foram obtidas as posterioris marginais para $\theta_{1}$ e $\theta_{2}$ usando o algoritmo D (Figura 4.9) considerando três valores para $\varepsilon$, onde os resultados obtidos quando $\varepsilon$ foi tomado de modo a aceitar $10 \%$ das simulações foram os piores. Tomando as amostras correspondentes a este valor alto de $\varepsilon$ e aplicando as técnicas de pós processamento de Beaumont et al. (2002) e Blum e François (2010), é possível obter a Figura 4.10. Observe que mesmo tomando um valor relativamente grande para $\varepsilon$, os métodos de pós-processamento são capazes de tornar os resultados tão precisos quanto os obtidos com valores menores.

Exemplo 7 - Distribuição $g$-and- $k$. Vários conjuntos de dados complexos não admitem distribuições padrão como modelo, muitas vezes sugerindo distribuições assimétricas, leptocúrticas ou platicúrticas. Tais dados podem ser modelados por distribuições da família de distribuições quantílicas, que possuem formas flexíveis. Os membros dessa família são definidos pelo inverso de suas funções de distribuição acumulada e raramente possuem verossimilhança analiticamente tratável, tornando os métodos ABC uma ferramenta potencialmente viável nesses casos. A distribuição $g$-and$k$ (Haynes et al., 1997) é definida por sua função de distribuição inversa (4.8) e não possui densidade dada analíticamente, embora seja simples simular de tal distribuição através do método de inversão. A função de verossimilhança pode ser calculada numericamente (Rayner e MacGillivray, 2002), porém a um alto custo computacional.

A distribuição $g$-and- $k$ é definida por

$$
F^{-1}(x ; A, B, c, g, k)=A+B\left[1+c \frac{1-\exp \{-g z(x)\}}{1+\exp \{-g z(x)\}}\right]\left\{1+z(x)^{2}\right\}^{k} z(x),
$$

onde $z(x)$ é o $x$-ésimo quantil da distribuição normal padrão, $A$ e $B>0$ são parâmetros de localização e escala respectivamente, $g$ é um parâmetro relacionado à assimetria da distribuição, enquanto $k>-\frac{1}{2}$ está relacionado com a curtose. Em geral o parâmetro $c$ é fixo e igual a 0.8 para garantir 

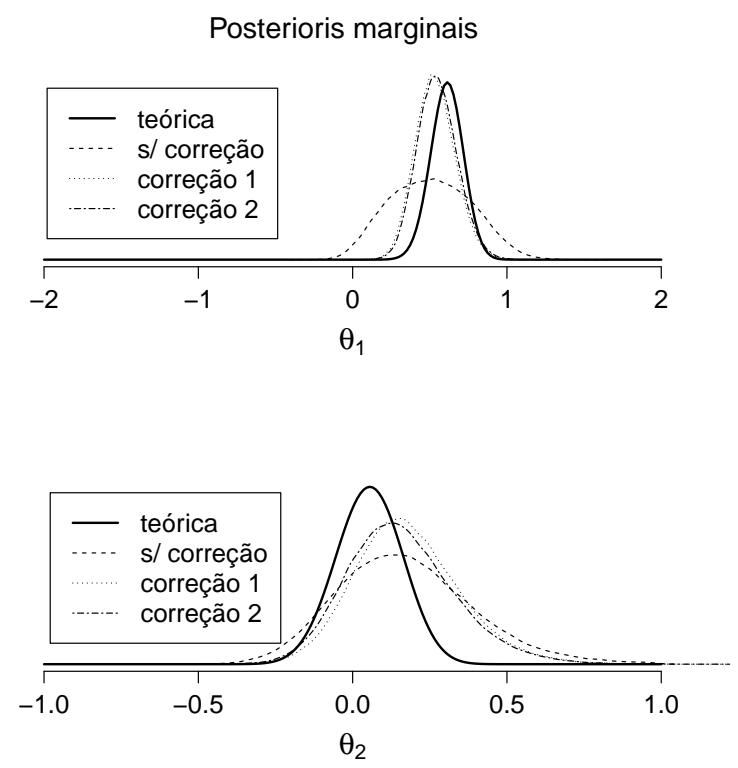

Figura 4.10: Distribuições a posteriori marginais de $\theta=\left(\theta_{1}, \theta_{2}\right)$ obtidas pelo algoritmo $D$. A curva sólida representa a distribuição teórica, enquanto as demais curvas correspondem as amostras obtidas sem pós processamento (s/ correção), com pós processamento de Beaumont et al. (2002) (correção 1) e de Blum e François (2010) (correção 2).

que a distribuição seja própria. Logo, $\theta=(A, B, g, k)$.

O uso do método ABC para estudar dados simulados da distribuição $g$-and- $k$ foi proposto em Allingham et al. (2009), onde é utilizado o algoritmo E. Os autores estudaram dados de tamanho $n=10^{4}$ com valor nominal de $\theta$ igual a $\theta_{0}=(3,1,2,0.5)$ e utilizaram para os parâmetros uma priori uniforme em $[0,10]^{4}$. O conjunto de estatísticas resumo considerado foi o conjunto de todas as estatísticas de ordem, ou seja, se $\mathcal{D}$ for o conjunto de dados com tamanho $n$, então $T(\mathcal{D})$ corresponde a uma ordenação crescente de todos os $n$ valores de $\mathcal{D}$. A métrica utilizada para medir a proximidade das estatísticas foi a norma Euclidiana e o kernel de transição proposto foi o Gaussiano truncado em $[0,10]^{4}$ com média igual ao estado atual da cadeia e variâncias iguais a 0.1 . O valor de $\varepsilon$ foi escolhido através de simulações, enquanto o valor inicial da cadeia foi obtido através de uma aplicação piloto do algoritmo D, utilizando-se as mesmas estatísticas resumo.

Embora não fique muito evidente, o método ABC proposto em Allingham et al. (2009) produz estimativas pobres se comparado com estimativas de máxima verossimilhança calculadas numericamente, especialmente para o parâmetro $g$. A Figura 4.11 mostra os níveis das densidades conjuntas de $(A, B)$ e $(g, k)$ obtidas por esse método (dois gráficos superiores) para uma amostra de tamanho 1000 com valor do parâmetro igual a $\theta_{0}$. Um intervalo de credibilidade de $95 \%$ para $g$ é igual a $(1.95 ; 9.77)$ e por pouco não contém o verdadeiro valor 2 .

Sob as mesmas condições descritas acima, Fearnhead e Prangle (2012) comparam os resultados obtidos em Allingham et al. (2009) com os obtidos pelo ABC semiautomático. Numa primeira análise, as variáveis explicativas do método semiautomático foram tomadas como sendo o conjunto completo de estatísticas de ordem e o ABC piloto do passo (SA1) é o próprio método de Allingham et al. (2009). Chamaremos este método de ABC semiauto I. A Figura 4.11 mostra os níveis das densidades conjuntas de de $(A, B)$ e $(g, k)$ obtidas segundo essa abordagem (dois gráficos inferiores) para a mesma amostra de tamanho 1000 considerada anteriormente. Neste caso, o intervalo de credibilidade de $95 \%$ para $g$ é igual a $(1.75 ; 4.04)$.

Uma escolha natural para as variáveis explicativas (função $h$ ) seria considerar as estatísticas de ordem e suas potências de 1 a 4, uma vez que os quatro parâmetros do modelo correspondem à localização, escala, assimetria e curtose. No entanto, tal escolha resulta no ajuste de um modelo linear com $4 \times 10^{4}$ variáveis explicativas (para amostras de tamanho $10^{4}$ ), o que torna o método 
pouco eficiente computacionalmente. Para melhorar a eficiência do método, Fearnhead e Prangle (2012) propõem utilizar um subconjunto de $m$ estatísticas de ordem igualmente espaçadas e $l$ potências das mesmas (que chamaremos de ABC semiauto II). Os valores de $m$ e $l$ foram escolhidos via $\mathrm{BIC}$, ajustando-se vários modelos lineares através da variação de $m$ em $\{60, \ldots, 140\}$ e $l$ em $\{1,2,3,4\}$. Neste caso foram obtidos $m=100$ e $l=4$. O ABC piloto do passo (SA1) foi realizado com esse mesmo conjunto de estatísticas através do algoritmo D.

Além disso, Fearnhead e Prangle (2012) usam o pós-processamento de Beaumont et al. (2002) na amostra a posteriori obtida em Allingham et al. (2009), que também é incluída na comparação (e que será chamada de Allingham + correção).

A comparação foi realizada através do erro quadrático médio, utilizando-se 50 amostras aleatórias de tamanho $n=10^{4}$ da distribuição $g$-and- $k$ com parâmetro igual a $\theta_{0}=(3,1,2,0.5)$. Os resultados são dados na Tabela 4.5 onde fica evidente a melhor performance dos métodos ABC semiautomáticos, especialmente sob a abordagem ABC semiauto II que retorna resultados idênticos aos obtidos por máxima verossimilhança, que foram obtidas numericamente como em Rayner e MacGillivray (2002).
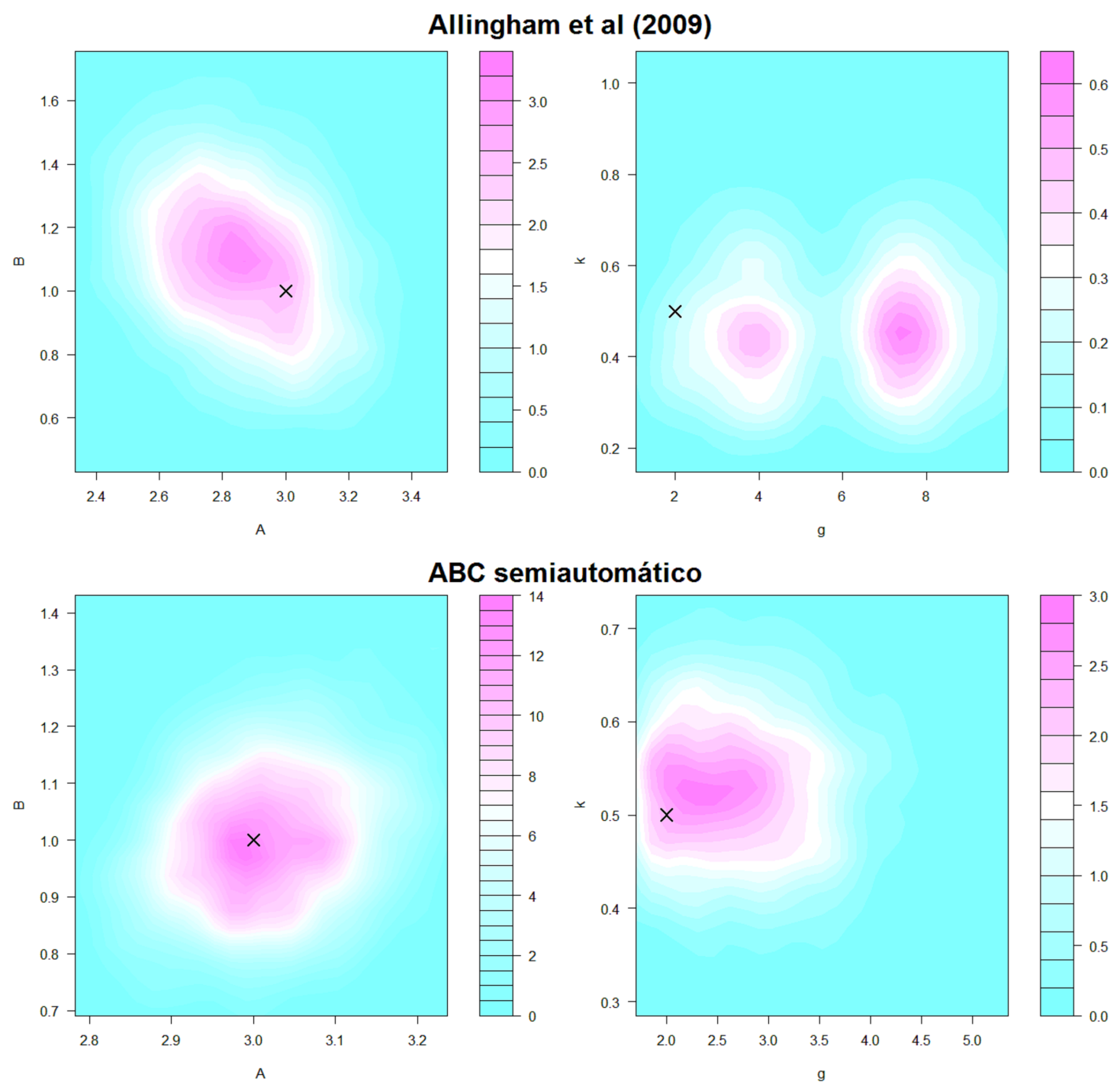

Figura 4.11: Niveis das densidades conjuntas para $(A, B)$ (esquerda) e $(g, k)$ (direita) utilizando o $A B C$ proposto por Allingham et al. (2009) (gráficos superiores) e o método ABC semiautomático de Fearnhead e Prangle (2012) (gráficos inferiores) para uma amostra de tamanho 1000 com valor nominal de $\theta$ igual a $\theta_{0}=(3,1,2,0.5)$. $O$ " $\times$ " demarca o valor nominal. 


\begin{tabular}{l|cccc}
\hline \hline Método & $A$ & $B$ & $g$ & $k$ \\
\hline Allingham et al (2009) & 0.00590 & 0.00130 & 3.8500 & 0.00063 \\
Allingham + correção & 0.00040 & 0.00170 & 0.2800 & 0.00051 \\
ABC semiauto I & 0.00016 & 0.00056 & 0.0441 & 0.00023 \\
ABC semiauto II & 0.00015 & 0.00053 & 0.0014 & 0.00015 \\
Máx. verossimilhança & 0.00016 & 0.00055 & 0.0013 & 0.00014 \\
\hline \hline
\end{tabular}

Tabela 4.5: Erro quadrático médio das estimativas baseadas em 50 simulações de Monte Carlo da distribuição g-and-k de tamanho $n=10^{4}$ e parâmetro nominal igual a $\theta_{0}=(3,1,2,0.5)$, segundo cada abordagem.

\subsection{Uma nota sobre a escolha de modelos e testes de hipóteses através do ABC}

Seja $\mathcal{D}$ um conjunto de dados em um espaço amostral $\mathcal{X}$ modelado por $f(\cdot \mid \theta)$, com $\theta \in \Theta$. No contexto Bayesiano, um objeto padrão para a comparação de modelos é a verossimilhança marginal (Jeffreys, 1939)

$$
\nu(\mathcal{D})=\int_{\Theta} \pi(\theta) f(\mathcal{D} \mid \theta) \theta,
$$

onde $\pi(\theta)$ é a distribuição a priori de $\theta$.

O fator de Bayes, definido por

$$
B_{12}(\mathcal{D})=\frac{\int_{\Theta_{1}} \pi_{1}\left(\theta_{1}\right) f_{1}\left(\mathcal{D} \mid \theta_{1}\right) d \theta_{1}}{\int_{\Theta_{2}} \pi_{2}\left(\theta_{2}\right) f_{2}\left(\mathcal{D} \mid \theta_{2}\right) d \theta_{2}}=\frac{\nu_{1}(\mathcal{D})}{\nu_{2}(\mathcal{D})}
$$

utiliza o objeto (4.9) para comparar as evidências de dois modelos, cada qual com verossimilhanças $f_{1}\left(\mathcal{D} \mid \theta_{1}\right)$ e $f_{2}\left(\mathcal{D} \mid \theta_{2}\right)$ (Robert, 2007).

A escolha Bayesiana de modelos tem como objetivo criar uma estrutura probabilística através de $M$ modelos (ou verossimilhanças). Neste contexto, dados $M$ modelos dos quais um deva ser escolhido, devemos considerar uma priori $\pi(\mathcal{M}=m)(m=1, \ldots, M)$ de que o verdadeiro modelo seja o m-ésimo, além das prioris para os parâmetros condicional a $\mathcal{M}=m$, denotadas por $\pi_{m}\left(\theta_{m}\right)$ e definidas em $\Theta_{m}$. Assim, $\mathcal{M}$ passa a ser considerado um parâmetro e a escolha do modelo é orientada pela distribuição a posteriori de $\mathcal{M}$

$$
\mathbb{P}(\mathcal{M}=m \mid \mathcal{D})=\frac{\pi(\mathcal{M}=m) \nu_{m}(\mathcal{D})}{\sum_{k} \pi(\mathcal{M}=m) \nu_{k}(\mathcal{D})},
$$

onde $\nu_{k}(\mathcal{D})=\int_{\Theta_{k}} \pi_{k}\left(\theta_{k}\right) f_{k}\left(\mathcal{D} \mid \theta_{k}\right) d \theta_{k}$ é a verossimilhança marginal para o k-ésimo modelo.

Embora a obtenção desta posteriori seja, em geral, complicada do ponto de vista prático, os métodos $\mathrm{ABC}$ fornecem uma solução simples, especialmente em casos onde a verossimilhança não está disponível. Logo, como apontado em Grelaud et al. (2009) e Didelot et al. (2011), o espaço paramétrico é estendido, isto é, $\Theta=\cup_{m=1}^{M}\{m\} \times \Theta_{m}$ e a aproximação para sua posteriori pode ser obtida através dos métodos $\mathrm{ABC}$ vistos até agora. Assim, uma implementação de um método $\mathrm{ABC}$ para escolha de modelos é dada por:

\footnotetext{
Algoritmo G: ABC-MC ou ABC escolha de modelo

G1: Simule $m$ da priori $\pi(\mathcal{M})$,

G2: simule $\theta_{m}$ da priori $\pi_{m}\left(\theta_{m}\right)$,

G3: simule $\mathcal{D}^{\prime}$ do $m$-ésimo modelo com o parâmetro $\theta_{m}$ simulado em G2,

G4: calcule a distância $d=d\left(\mathcal{D}, \mathcal{D}^{\prime}\right)$ entre $\mathcal{D}$ e $\mathcal{D}^{\prime}$,

G5: aceite $m$ e $\theta_{m}$ se $d \leq \varepsilon$; retorne a G1.
} 
A estimativa $\mathrm{ABC}$ da probabilidade a posteriori $\mathbb{P}(\mathcal{M}=m \mid \mathcal{D})$ é a frequência de aceitação do $m$-ésimo modelo, que também corresponde à proporção de dados simulados que estão próximos dos dados observados $\mathcal{D}$ segundo a tolerância $\varepsilon>0$ suficientemente pequena.

Novamente, é importante observar que na prática o passo G4 do algoritmo G não é muito eficiente, por isso, devemos recorrer ao uso de estatísticas resumo, substituindo este passo por

G4 $4^{\prime}$ : Calcule $\mathbf{T}^{\prime}=\mathbf{T}\left(\mathcal{D}^{\prime}\right)$ e a distância $d=d\left(\mathbf{T}, \mathbf{T}^{\prime}\right)$ entre $T$ e $T^{\prime}$,

onde o conjunto de estatísticas resumo $\mathbf{T}=\mathbf{T}(\mathcal{D})=\left(T^{1}(\mathcal{D}), \ldots, T^{M}(\mathcal{D})\right)$ é uma concatenação das estatísticas resumo utilizadas em todos os modelos (eliminando-se as duplicatas). Neste caso, a proporção de dados simulados que estão próximos dos dados observados $\mathcal{D}$ segundo a tolerância $\varepsilon>0$ suficientemente pequena, estima a probabilidade a posteriori $\pi(\mathcal{M}=m \mid \mathbf{T}(\mathcal{D})) \neq \pi(\mathcal{M}=m \mid \mathcal{D})$ quando $\mathbf{T}$ é insuficiciente.

A estimativa do fator de Bayes (4.10) obtida pelo algoritmo G com o passo G4' é dada por

$$
\widehat{B_{12}}(\mathcal{D})=\frac{\pi(\mathcal{M}=2) \sum_{l=1}^{L} \mathbb{I}\left\{m^{l}=1\right\} \mathbb{I}\left\{d\left(\mathbf{T}, \mathbf{T}^{l}\right) \leq \varepsilon\right\}}{\pi(\mathcal{M}=1) \sum_{l=1}^{L} \mathbb{I}\left\{m^{l}=2\right\} \mathbb{I}\left\{d\left(\mathbf{T}, \mathbf{T}^{l}\right) \leq \varepsilon\right\}},
$$

onde $L$ é o número necessário de simulações para se obter um dado número de aceitações, $\mathbf{T}^{l}=$ $\mathbf{T}\left(\mathcal{D}^{l}\right)$ e os pares $\left(m^{l}, \mathcal{D}^{l}\right)$ são simulados da priori conjunta.

Quando métodos $\mathrm{ABC}$ são utilizados para estimação pontual, o uso de estatísticas insuficientes não invalida os resultados obtidos, como pode ser visto em Fearnhead e Prangle (2012). Por outro lado, a perda de informação induzida pela estatística $\mathbf{T}$, em geral, tem um efeito significativo na convergência de (4.11) para o seu verdadeiro valor, mesmo quando $\mathbf{T}$ é suficiente para ambos os modelos, como demonstrado em Robert et al. (2011). A convergência de (4.11) para $B_{12}(\mathcal{D})$ só é válida para um número muito pequeno de casos conhecidos, como nos campos aleatórios de Gibbs (Gibbs random fields) estudados em Grelaud et al. (2009), onde cada campo aleatório possui sua própria estatística suficiente, que também é conjuntamente suficiente para $\left(\mathcal{M}, \theta_{1}, \ldots, \theta_{m}\right)$. O Exemplo 8, a ser apresentado posteriormente, ilustra essa deficiência do uso do ABC na escolha de modelos.

Definição 4.6 (Hipóteses precisas). Uma hipótese $H$ que determina que $\theta$ pertence a um subconjunto $\Theta_{H}$ de dimensão menor que $\Theta$, é dita hipótese precisa.

Como um subproduto dos métodos $\mathrm{ABC}$, é possível calcular (aproximadamente) o $e$-valor, uma medida de evidência definida em Pereira e Stern (1999) para testar hipóteses precisas. Os testes de hipóteses baseados no e-valor também são conhecidos por FBST de Full Bayesian Significance Test e serão definidos a seguir (Pereira et al. (2008) apresentam uma revisão do FBST).

Definição 4.7 (e-valor). Considere uma hipótese precisa $H: \theta \in \Theta_{H}$ e sejam

$$
\pi_{0}=\sup _{H} f(\theta \mid \mathcal{D}) \quad e \quad T_{0}=\left\{\theta \in \Theta: f(\theta \mid \mathcal{D})>\pi_{0}\right\} .
$$

$O$ valor de evidência Bayesiano contra $H$, denotado por êv, é definido como a seguinte probabilidade a posteriori

$$
\overline{e v}=\mathbb{P}\left(\theta \in T_{0} \mid \mathcal{D}\right)=\int_{T_{0}} f(\theta \mid \mathcal{D}) d \theta .
$$

O conjunto $T_{0}$ é comumente chamado de conjunto tangente a $\Theta_{H}$. Como a hipótese alternativa não é precisa, temos que $\overline{e v}$ não é a evidência em favor da hipótese alternativa. Equivalentemente, temos que $e v=1-\overline{e v}$ é a hipótese em favor de $H$ mas não é a evidência contra a hipótese alternativa. A probabilidade $e v$ também é chamada de $e$-valor e seu uso não viola o chamado Princípio da Verossimilhança (veja Basu (1975) e Birnbaum (1962)).

Definição 4.8 (FBST). O teste FBST é o teste que rejeita $H$ sempre que ev $=1-\bar{v}$ é pequeno. 
O FBST satisfaz requerimentos lógicos que não são satisfeitos pelos $p$-valores e pelos testes que baseiam-se nos fatores de Bayes (Stern, 2003). Além disso, sempre que a distribuição a posteriori é absolutamente contínua e a hipótese nula é precisa, o uso de fatores de Bayes para testes de significância é controverso, como apontado nos trabalhos de Good (1983), Lindley (1957), Lindley (1997) e Shafer (1982).

Exemplo 8 - Processo de médias móveis (revisitado - parte 2). Voltando ao exemplo do modelo MA(2), utilizamos o algoritmo G para escolher entre os modelos MA(1) e MA(2) com base nos dados. Neste caso, as probabilidades a posteriori de cada modelo podem ser obtidas através de integração numérica e seus valores são aproximadamente iguais a 0.0530 para o modelo MA(1) e 0.9470 para o modelo MA(2).

As probabilidades a posteriori de cada modelo obtidas pelo algoritmo G são dadas na Tabela 4.6 onde consideramos diferentes valores para a precisão $\varepsilon$ do algoritmo. Note que a probabilidade a posteriori para o modelo $\mathrm{MA}(2)$ subestima seu valor verdadeiro e aumenta pouco conforme a precisão diminui. O contrário acontece com a probabilidade a posteriori do modelo MA(1). Mesmo com um número muito grande de simulações (aqui usamos $10^{6}$ ), o ganho na aproximação é muito pequeno, pois a convergência é muito lenta.

Essa discrepância mostra a limitação do uso do ABC para aproximar os fatores de Bayes como exposto em Robert et al. (2011).

\begin{tabular}{ccc}
\hline \hline$\varepsilon$ & $\hat{\mathbb{P}}(\mathcal{M}=1 \mid \mathcal{D})$ & $\hat{\mathbb{P}}(\mathcal{M}=2 \mid \mathcal{D})$ \\
\hline$q_{10 \%}(d)$ & 0.2352 & 0.7648 \\
$q_{1 \%}(d)$ & 0.2256 & 0.7744 \\
$q_{0.1 \%}(d)$ & 0.2190 & 0.7810 \\
\hline Nominal & 0.0530 & 0.9470 \\
\hline \hline
\end{tabular}

Tabela 4.6: Estimativas para as probabilidades a posteriori de cada modelo obtidas através do algoritmo $G$ considerando diferentes valores para $\varepsilon$. O valor $q_{p \%}(d)$ indica o número de simulações aceitas. A última linha indica os valores verdadeiros de $\mathbb{P}(\mathcal{M}=m \mid \mathcal{D})$ obtidos por integração numérica.

O próximo exemplo é uma abordagem original à um problema de estimação que surge naturalmente no contexto de processos com memória longa.

Exemplo 9 - Distribuição de Rosenblatt. A distribuição de Rosenblatt é a distribuição não Gaussiana mais simples que surge como limite não degenerado de somas parciais de variáveis aleatórias com dependência de longo alcance. Tal distribuição está relacionada com o processo de Rosenblatt (apresentado na seção 2.2) como sendo a distribuição desse processo com parâmetro de memória $H \in(3 / 4,1)$ no instante $t=1$. A distribuição de Rosenblatt também surge como limite de alguns estimadores no contexto de inferência em processos com memória longa.

Seja $\sigma_{D}=\{(1-2 D)(1-D) / 2\}^{1 / 2}$, com $D \in(0,1 / 2)$. Rosenblatt (1961) demonstra que se $\left\{X_{i}\right\}_{i \in \mathbb{N}}$ é uma sequência estacionária Gaussiana com estrutura de covariância dada por $E X_{0} X_{k} \sim$ $k^{-D}$ quando $k \rightarrow \infty$ então

$$
Z_{D}^{N}=\frac{\sigma_{D}}{N^{1-D}} \sum_{i=1}^{N}\left(X_{i}^{2}-1\right),
$$

converge, quando $N \rightarrow \infty$, para uma v.a. não degenerada com média 0 e variância 1 , que denotaremos por $Z_{D}$. A distribuição de $Z_{D}$ recebeu o nome de distribuição de Rosenblatt em Taqqu (1975). O fator de escala $\sigma_{D}$ garante que $E\left(Z_{D}\right)^{2}=1$ (Taqqu, 2011).

As funções de distribuição e densidade de $Z_{D}$ não possuem forma analítica, mas $Z_{D}$ pode ser caracterizada por sua função característica (que é convergente apenas próximo da origem) dada por 


$$
\phi(t)=\exp \left\{\sum_{k=2}^{\infty} \frac{\left(2 i t \sigma_{D}\right)^{k} c_{k}}{2 k}\right\}, t \in \mathbb{R}
$$

onde

$$
c_{k}=\int_{x \in[0,1]^{k}}\left|x_{k}-x_{1}\right|^{-D} \prod_{j=2}^{k}\left|x_{j-1}-x_{j}\right|^{-D} d x,
$$

ou pode ser caracterizada como limite da soma ponderada de variáveis qui-quadrado padronizadas, isto é,

$$
Z_{D}=\sum_{n=1}^{\infty} \lambda_{n}(D)\left(\epsilon_{n}^{2}-1\right),
$$

onde $\epsilon_{n} \sim N(0,1)(n=1,2, \ldots)$ e os valores $\lambda_{n}(D)$ devem satisfazer a relação $\sum_{n=1}^{\infty} \lambda_{n}(D)^{k}=\sigma_{D}^{k} c_{k}$ $(k=2,3, \ldots)$.

Quando $D \rightarrow 0^{+}$, temos que $c_{k} \rightarrow 1$ para todo $k$ e $\sigma_{D} \rightarrow 1 / \sqrt{2}$, deste modo, para $t$ suficientemente pequeno temos que

$$
\begin{aligned}
\phi(t) & =\exp \left\{\frac{1}{2} \sum_{k=2}^{\infty} \frac{(\sqrt{2} i t)^{k}}{k}\right\} \\
& =\exp \left\{\frac{1}{2}\left[\sum_{k=1}^{\infty} \frac{(\sqrt{2} i t)^{k}}{k}-\sqrt{2} i t\right]\right\} \\
& =\exp \left\{\frac{1}{2}[-\log (1-\sqrt{2} i t)-\sqrt{2} i t]\right\} \\
& =\left(\frac{1}{1-\sqrt{2} i t}\right)^{1 / 2} e^{-i t / \sqrt{2}}
\end{aligned}
$$

que é a função característica da v.a. $\frac{1}{\sqrt{2}}\left(\epsilon^{2}-1\right)$, com $\epsilon \sim N(0,1)$, ou seja, $Z_{0}$ é uma v.a. qui-quadrado com 1 grau de liberdade, padronizada para ter média 0 e variância 1.

Quando $D \rightarrow 1 / 2^{-}$, o denominador do fator de escala em (4.12) tende a $\sqrt{N}$ e podemos intuitivamente assumir, pelo teorema central do limite, que $Z_{1 / 2} \sim N(0,1)$. Uma demonstração formal desse fato é dada em Veillette et al. (2013) através de uma representação de Lévy-Khintchine de $Z_{D}$, o que torna possível a obtenção de uma forma alternativa para a função característica (4.13), onde o limite Gaussiano é mais simples de ser obtido.

A representação dada por (4.14) possibilita a simulação de amostras aleatórias da distribuição de Rosenblatt, desde que seja possível calcular uma grande quantidade dos valores $\lambda_{n}(D)$. Veillette et al. (2013) demonstram que esses valores podem ser considerados autovalores de um determinado operador linear, tornando possível calculá-los numericamente. Além disso, os $\lambda_{n}(D)$ são não crescentes em $n$ e convergem para zero. A Tabela 4.7 fornece os primeiros 10 valores de $\lambda_{n}(D)$, bem como $\lambda_{500}(D)$ com $D \in\{0.1,0.2,0.3,0.4\}$ obtidos numericamente como em Veillette et al. (2013).

A partir da expansão em autovalores (4.14) de $Z_{D}$, Veillette et al. (2013) derivam métodos numéricos para obtenção dos cumulantes, momentos, função de distribuição e densidade da distribuição de Rosenblatt. A Figura 4.12 apresenta as funções de distribuição e densidade de $Z_{D}$ obtidas numericamente para alguns valores de $D$, onde fica evidente os casos limites $D=0$ e $D=1 / 2$.

Embora seja possível calcular a densidade de $Z_{D}$ numericamente, o tempo computacional gasto para calcular tal densidade avaliada em um número grande de valores torna a implementação de métodos de máxima verossimilhança pouco eficientes. Tentamos fazê-lo utilizando o mesmo código MATLAB utilizado em Veillette et al. (2013) e o tempo gasto para calcular uma única estimativa de 


\begin{tabular}{ccccc}
\hline \hline $\mathrm{n}$ & $D=0.1$ & $D=0.2$ & $D=0.3$ & $D=0.4$ \\
\hline 1 & 0.7020 & 0.6813 & 0.6305 & 0.5125 \\
2 & 0.0565 & 0.1126 & 0.1604 & 0.1784 \\
3 & 0.0348 & 0.0734 & 0.1107 & 0.1301 \\
4 & 0.0245 & 0.0541 & 0.0851 & 0.1042 \\
5 & 0.0195 & 0.0441 & 0.0712 & 0.0895 \\
6 & 0.0160 & 0.0371 & 0.0613 & 0.0788 \\
7 & 0.0137 & 0.0324 & 0.0545 & 0.0712 \\
8 & 0.0120 & 0.0287 & 0.0490 & 0.0651 \\
9 & 0.0107 & 0.0260 & 0.0449 & 0.0604 \\
10 & 0.0096 & 0.0237 & 0.0414 & 0.0563 \\
500 & $8.1 \times 10^{-5}$ & $3.3 \times 10^{-4}$ & $9.9 \times 10^{-4}$ & $2.3 \times 10^{-3}$ \\
\hline \hline
\end{tabular}

Tabela 4.7: Primeiros 10 valores de $\lambda_{n}(D)$ e $\lambda_{500}(D)$ para diferentes valores de $D$ obtidos numericamente.
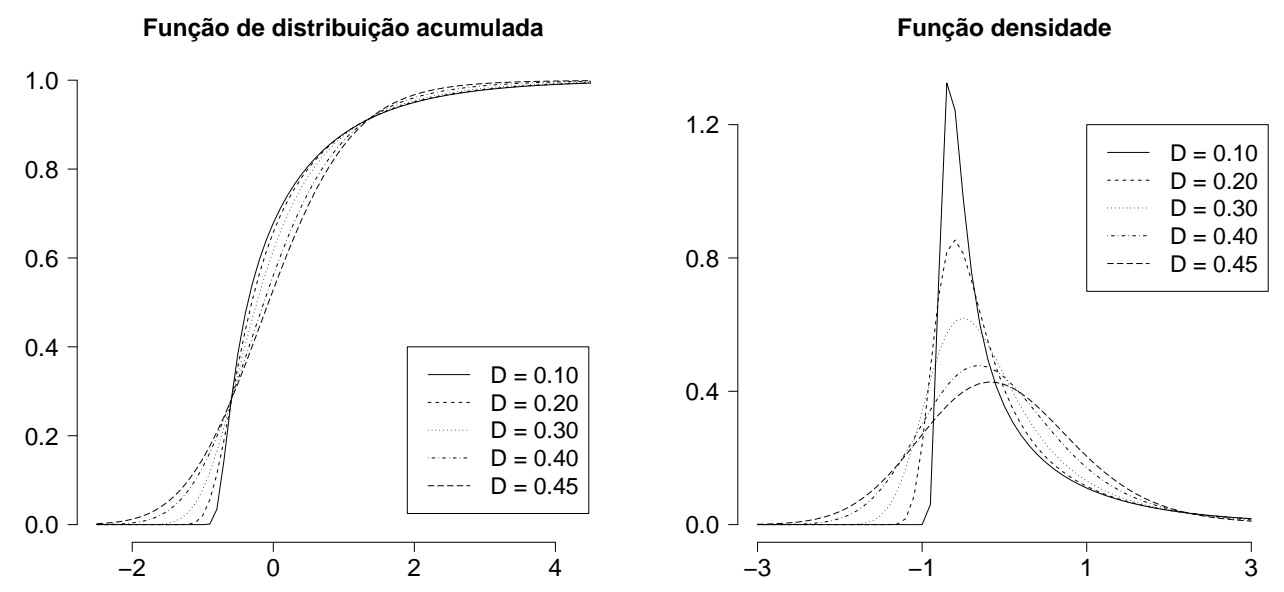

Figura 4.12: Gráficos da função de distribuição (esquerda) e função de densidade (direita) da distribuição de Rosenblatt para alguns valores de D.

máxima verossimilhança para uma amostra de tamanho 500 foi de quase 4 horas em um computador comum. No entanto, a simulação de amostras aleatórias utilizando a expansão (4.14) é precisa e eficiente, o que torna os métodos $\mathrm{ABC}$ uma alternativa viável para se fazer inferência para essa distribuição. Vamos utilizar essa expansão para simular amostras da distribuição de Rosenblatt e aplicar o método ABC de diferentes maneiras. Em todos os casos a distribuição a priori para $D$ será uniforme contínua no intervalo $(0,1 / 2)$.

Em um primeiro momento consideramos o uso do algoritmo D para obter uma amostra aproximada a posteriori para o parâmetro D da distribuição de Rosenblatt. Como vimos no exemplo 7, que tratava da distribuição $g$-and- $k$, uma escolha natural consiste em considerar como estatísticas resumo o conjunto completo de estatísticas de ordem. Da mesma forma, vamos tomar a norma Euclidiana como métrica do algoritmo D nesta etapa. A Figura 4.13 apresenta os boxplots das estimativas obtidas (média a posteriori aproximada) nas condições acima, para 100 simulações de Monte Carlo de $Z_{D}$ variando $D$ em $\{0.1,0.2,0.3,0.4\}$. O valor de $\varepsilon$ foi novamente tomado de modo a aceitar $1 \%$ das amostras simuladas em 10000 iterações do algoritmo. Note que a distribuição amostral das estimativas indicam que o método $\mathrm{ABC}$ descrito acima é capaz de retornar resultados razoáveis, mesmo para um número baixo de iterações. Isso é esperado uma vez que as estatísticas que utilizamos baseia-se em toda a amostra que por sua vez é uma estatística suficiente. A Tabela 4.9 apresenta o erro quadrático médio (EQM) dessas estimativas. A mesma tabela fornece os resultados desse mesmo método $\mathrm{ABC}$ com a técnica de pós-processamento de Beaumont et al. (2002) apresentado na seção 4.2. Neste caso utilizamos $m=100$ estatísticas de ordem igualmente 
espaçadas como estatísticas resumo e nota-se um leve ganho em precisão. O aumento do valor de $m$ não melhora as estimativas significativamente.

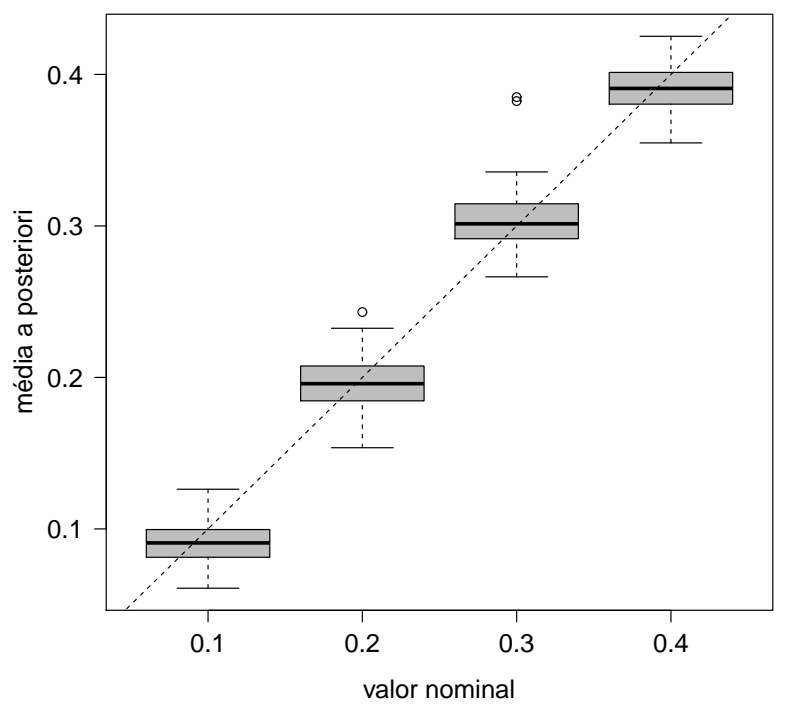

Figura 4.13: Distribuição amostral da média a posteriori para D obtidas pelo algoritmo D. Cada coluna corresponde a um valor nominal utilizado nas simulações.

Vamos agora considerar o uso do algoritmo D porém utilizando métricas diferentes da Euclidiana. Nesta etapa, ao invés de calcular a distância Euclidiana entre estatísticas resumo diversas, vamos calcular a distância entre funções de distribuição, utilizando métricas como a distância de Kolmogorov-Smirnov

$$
d_{K S}\left(F_{1}, F_{2}\right)=\sup _{x}\left|F_{1}(x)-F_{2}(x)\right|,
$$

a discrepância de Cramér-von Mises

$$
d_{C M}\left(F_{1}, F_{2}\right)=\int\left(F_{1}(x)-F_{2}(x)\right)^{2} d F_{1}(x),
$$

a divergência de Kullback-Leibler

$$
d_{K L}\left(F_{1}, F_{2}\right)=\int \log \left(\frac{f_{1}(x)}{f_{2}(x)} f_{1}(x) d x\right),
$$

e por último, a distância de Hellinger,

$$
H^{2}\left(F_{1}, F_{2}\right)=1-\int \sqrt{f_{1}(x) f_{2}(x)} d x
$$

onde $f_{i}$ é a densidade associada a função de distribuição acumulada $F_{i}(i=1,2)$.

Todas essas distâncias foram estimadas utilizando-se de suas versões discretas em pacotes disponíveis em $\mathrm{R}$ e MATLAB.

Novamente realizamos 100 simulações de Monte Carlo de $Z_{D}$ para cada valor de $D$ em $\{0.1,0.2,0.3,0.4\}$, e aplicamos o algoritmo D para cada amostra (10000 iterações) aceitando 1\% das simulações. A Tabela 4.8 fornece a média e o desvio-padrão das estimativas obtidas (média a posteriori aproximada) para cada valor nominal de D utilizado nas simulações. Nota-se que este método ABC performa bem para todas as distâncias utilizadas com uma leve vantagem para a distância de Hellinger. A Tabela 4.9 apresenta o erro quadrático médio das estimativas considerando apenas a distância de Hellinger. 


\begin{tabular}{lcccc}
\hline \hline $\mathbf{D}$ & Kolmogorov-Smirnov & Cramér-von Mises & Kullback-Leibler & Hellinger \\
\hline $\mathbf{0 . 1}$ & $0.1016(0.0065)$ & $0.1008(0.0068)$ & $0.1062(0.0046)$ & $0.1001(0.0054)$ \\
$\mathbf{0 . 2}$ & $0.2027(0.0109)$ & $0.2002(0.0100)$ & $0.2091(0.0094)$ & $0.2006(0.0066)$ \\
$\mathbf{0 . 3}$ & $0.3110(0.0209)$ & $0.3070(0.0186)$ & $0.3259(0.0105)$ & $0.3027(0.0130)$ \\
$\mathbf{0 . 4}$ & $0.3952(0.0165)$ & $0.3935(0.0125)$ & $0.3708(0.0111)$ & $0.3958(0.0110)$ \\
\hline \hline
\end{tabular}

Tabela 4.8: Média e desvio padrão amostral das estimativas para D em 100 simulações de Monte Carlo para cada métrica considerada.

Por último, consideramos o uso do método ABC semiautomático de Fearnhead e Prangle (2012) apresentado na seção 4.1. Como o parâmetro $D$ pertence a um pequeno intervalo da reta, o passo (SA1) é desnecessário. Escolhemos a mesma função $h$ utilizada no exemplo 7, isto é, $h$ mapeia toda a amostra em $m=100$ estatísticas de ordem igualmente espaçadas e suas potências de ordem 2 a 4. Assim como no método de pós-processamento, o aumento do valor de $m$ não melhora as estimativas significativamente. Como sempre, $\varepsilon$ foi escolhido de modo a aceitar $1 \%$ das 10000 simulações do algoritmo. A Figura 4.14 apresenta as posterioris aproximadas obtidas por esse método para quatro amostras típicas da distribuição de Rosenblatt, cada qual com parâmetros 0.1, 0.2, 0.3 e 0.4 onde nota-se que os resultados obtidos correspondem ao esperado. A Tabela 4.9 apresenta o erro quadrático médio das estimativas obtidas (média a posteriori aproximada) em 100 simulações de Monte Carlo para diferentes valores de $D$.

(a)

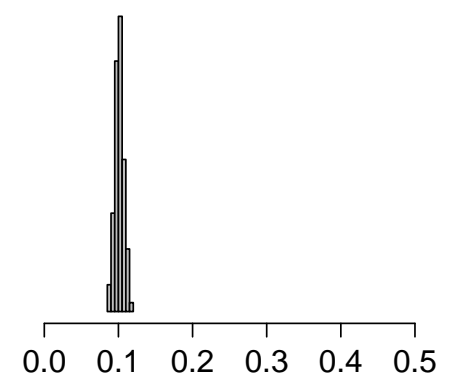

(c)

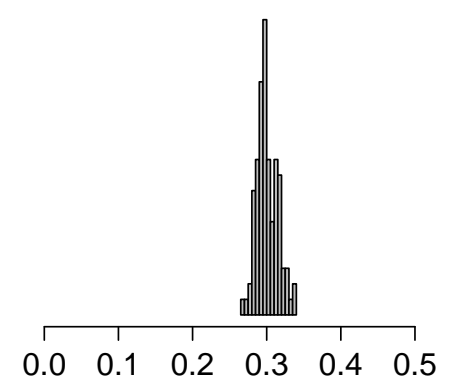

(b)

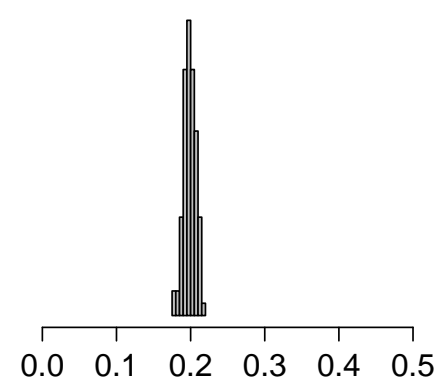

(d)

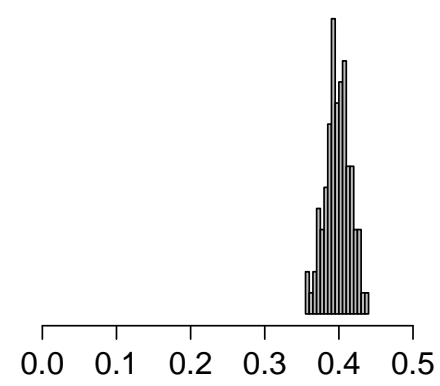

Figura 4.14: Distribuição a posteriori para $D$ obtido pelo $A B C$ semiautomático para uma amostra de tamanho 1000 da distribuição de Rosenblatt quando: (a) $D=0.1$, (b) $D=0.2$, (c) $D=0.3$ e (d) $D=0.4$.

Como é possível observar na Tabela 4.9, todas as abordagens retornaram resultados satisfatórios com uma vantagem negligenciável daquela que utiliza a distância de Hellinger como medida de discrepância no algoritmo D. No entanto, essa mesma abordagem é a que apresenta maior custo computacional (em média 130 segundos para obter cada estimativa), uma vez que deve-se estimar 


\begin{tabular}{l|cccc}
\hline \hline Algoritmo & $\mathbf{0 . 1}$ & $\mathbf{0 . 2}$ & $\mathbf{0 . 3}$ & $\mathbf{0 . 4}$ \\
\hline D: estatísticas de ordem & $3.1 \times 10^{-4}$ & $2.7 \times 10^{-4}$ & $3.9 \times 10^{-4}$ & $3.0 \times 10^{-4}$ \\
D: pós-processamento & $3.9 \times 10^{-5}$ & $7.7 \times 10^{-5}$ & $1.9 \times 10^{-4}$ & $1.7 \times 10^{-4}$ \\
D: distância de Hellinger & $2.9 \times 10^{-5}$ & $4.3 \times 10^{-5}$ & $1.7 \times 10^{-4}$ & $1.0 \times 10^{-4}$ \\
ABC semiautomático & $3.5 \times 10^{-5}$ & $6.5 \times 10^{-5}$ & $2.1 \times 10^{-4}$ & $2.0 \times 10^{-4}$ \\
\hline \hline
\end{tabular}

Tabela 4.9: Erro quadrático médio (EQM) das estimativas obtidas (média a posteriori aproximada) em 100 simulações de Monte Carlo segundo diferentes aplicações do método $A B C$.

a função de distribuição acumulada e a densidade das amostras simuladas em cada iteração do algoritmo D. A abordagem com menor custo computacional corresponde ao algoritmo ABC semiautomático onde cada estimativa foi obtida em um tempo médio de 3 segundos. Nesse custo computacional não foi incluído o tempo gasto para simular as amostras utilizadas em todos os métodos $\mathrm{ABC}$ considerados, uma vez que essas amostras precisam ser simuladas uma única vez e podem ser reutilizadas sempre que uma nova amostra for observada.

Consideramos o uso do ABC semiautomático para obter numericamente os e-valores (seção 4.3) associados às hipóteses $D_{0}=0$ e $D_{0}=0.5$ quando o valor nominal das amostras observadas viariam em uma grade de valores de $D$. Lembre que quando $D=0$ a distribuição de $Z_{D}$ é qui-quadrado com 1 grau de liberdade, padronizada para ter média 0 e variância 1 , enquanto que quando $D=0.5$ temos que $Z_{D} \sim N(0,1)$. Essas duas distribuições são bem conhecidas e fáceis de lidar na prática e correspondem à casos importantes da distribuição de Rosenblatt. Os e-valores foram obtidos através da integração numérica da densidade estimada por um kernel de Epanechnikov (4.7) construído com base na amostra aproximada a posteriori obtida pelo ABC semiautomático. Os gráficos da Figura 4.15 apresentam os e-valores calculados para cada hipótese em questão onde 100 amostras foram simuladas para cada valor de $D$. Note que em geral a evidência em favor da hipótese nula é maior do que para os demais valores de $D$ porém, é importante observar que o ABC performa mal nesses dois casos extremos, pois os valores simulados da priori para $D$ são sempre estritamente maiores que 0 e estritamente menores que 0.5 , tornando as posterioris obtidas nesses casos truncadas nesses valores. Isso explica o motivo de ocorrerem e-valores baixos, especialmente no caso $D_{0}=0.5$. Mesmo com essa limitação, a evidência em favor da hipótese nula será maior, em média, do que a evidência em favor dos demais valores. Este mesmo problema não acontece com os demais valores de $D$, mas estes não são interessantes do ponto de vista prático.

$\mathrm{D}_{0}=0.0$

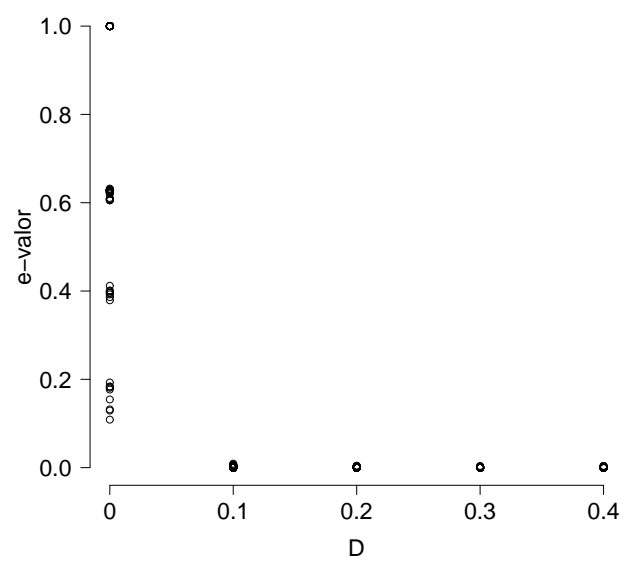

$\mathrm{D}_{0}=0.5$

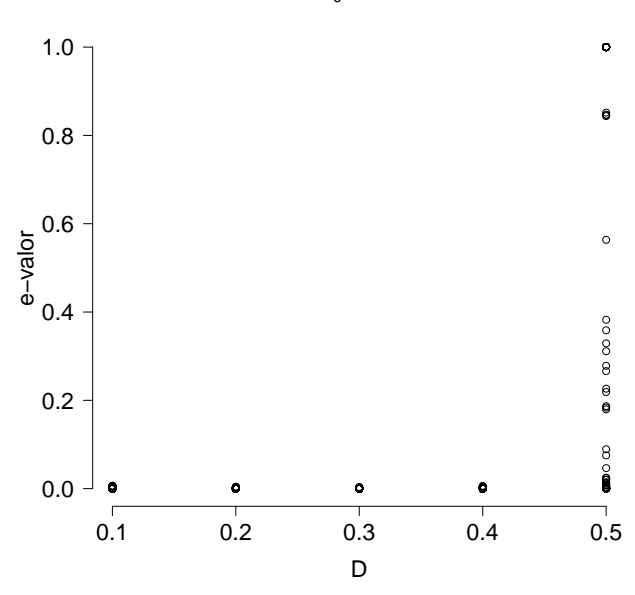

Figura 4.15: e-valores obtidos numericamente com respeito às hipóteses $D_{0}=0$ (esquerda) e $D_{0}=0.5$ (direita). 


\section{Capítulo 5}

\section{Aplicações e Conclusões}

Neste capítulo apresentaremos os resultados obtidos através de diferentes implementações do método ABC para os processos apresentados na seção 2.2. A performance de cada implementação será analisada a partir de simulações do modelo. Além disso, os resultados obtidos pela abordagem proposta serão comparados com os métodos de estimação clássicos apresentados no Capítulo 3.

\subsection{Descrição dos resultados}

O estudo de simulação foi realizado da mesma forma para todos os modelos como segue:

- Para cada valor nominal do parâmetro $\theta$, simulamos $K=200$ trajetórias do modelo denotadas por $\mathbf{x}^{k}=\left(x_{1}^{k}, \ldots, x_{n}^{k}\right)(k=1, \ldots, K)$, cada uma com comprimento $n=1000$.

- Para um dado método de estimação, foram obtidas $K$ estimativas para $\theta$, denotadas por $\left\{\theta_{k}, k=1, \ldots, K\right\}$.

- A partir dessas estimativas calculamos sua média, que será denotada por $\hat{\theta}$, seu desvio padrão, denotado por $d p$ e a raiz quadrada do erro quadrático médio definida por

$$
\sqrt{\mathrm{EQM}}:=\sqrt{\frac{1}{K} \sum_{k=1}^{K}\left(\theta_{k}-\theta\right)^{2}} .
$$

O valor $\sqrt{\mathrm{EQM}}$ fornece alguma informação sobre o viés do método de estimação quando comparado com $d p$.

As estatísticas utilizadas nos métodos $\mathrm{ABC}$ serão apresentadas em cada seção de acordo com cada modelo e o valor da tolerância $\varepsilon$ será sempre tomada de modo a aceitar $1 \%$ das simulações (20000 simulações). As estimativas consideradas serão dadas pela média das amostras obtidas por esses métodos (média aproximada a posteriori).

Os resultados serão apresentados em tabelas no Apêndice A e através de boxplots. Os boxplots fornecem uma visualização gráfica simples dos resultados obtidos com respeito à precisão e viés das estimativas.

\subsection{Ruído Gaussiano Fracionário}

Como visto na seção 2.2.2, o ruído Gaussiano fracionário (fGn) é o processo de incrementos do movimento Browniano fracionário. Por ser Gaussiano e autossimilar, todas as técnicas de estimação apresentadas no Capítulo 3 deveriam retornar estimativas razoáveis para o índice de memória, especialmente o estimador de Whittle que é obtido a partir de uma aproximação consistente da função de verossimilhança do fGn. Neste caso, temos que $\theta=H \operatorname{com} H \in(0,1)$. 
Inicialmente utilizamos o algoritmo D para obter uma posteriori aproximada para $H$. Para tanto, estudamos a performance de várias estatísticas resumo como:

1. $\log s^{2}(n) / \log (n) \operatorname{com} s^{2}(\cdot)$ dado em (3.2);

2. $\log R / S(n) / \log (n) \operatorname{com} R / S(\cdot)$ dado em (3.3);

3. $\log I_{n}[(n-1) / 2] / \log [(n-1) / 2] \operatorname{com} I_{n}(\cdot)$ dado em (3.4);

4. $\log F(n) / \log (n) \operatorname{com} F(\cdot)$ dado em (3.6);

5. variações quadráticas baseadas em filtros de diferença finita de ordem 1 a 5 , definidas na seção 3.7 ;

6. 100 estatísticas de ordem igualmente espaçadas começando pela $10^{\mathrm{a}}$ e suas potências de 2 a 4

7. todas as estatísticas de ordem e suas potências de 2 a 4 .

Consideramos também transformações como logarítmo, raíz quadrada e recíproca das estatísticas 1. a 5. pois, como apontado em Blum (2010), essas transformações podem melhorar a performance dos métodos ABC. A priori utilizada será sempre uniforme no intervalo $(0,1)$.

Vimos no Capítulo 3 que as estatísticas 1. a 5. são potencialmente informativas com respeito ao parâmetro $H$, pois seus valores mudam conforme $H$ muda e, por isso, são candidatas naturais à estatísticas resumo para o algoritmo $\mathrm{D}$, ou qualquer método $\mathrm{ABC}$ que utilize estatísticas resumo. Andrade e Rifo (2015a) consideram também o uso de mais duas estatísticas obtidas pelas análises KPSS e V/S (Beran et al., 2013), no entanto, o comportamento dessas estatísticas são similares ao comportamento da estatística $\mathrm{R} / \mathrm{S}$.

O uso das estatísticas de ordem e suas potências possuem informação com respeito à forma da distribuição marginal do processo e mostraram-se pouco eficazes, retornando estimativas distorcidas para $H$. Isso é esperado, pois a forma da distribuição não muda conforme o valor de $H$ muda (a distribuição marginal é sempre normal). Concluímos que o uso da própria série como estatística resumo (ou uma grande quantidade de estatísticas de ordem) fornece uma quantidade negligenciável de informação com respeito ao parâmetro de memória.

O uso das estatísticas 1. a 4. (e suas transformações) conjuntamente evidencia um certo aprendizado sobre o parâmetro $H$, mas ainda assim as estimativas apresentam viés e variabilidade consideráveis, tornando o método $\mathrm{ABC}$ baseado no algoritmo $\mathrm{D}$ insatisfatório frente a seus competidores clássicos. O uso de pós-processamento da amostra a posteriori obtida não melhora esses resultados.

Utilizamos o critério de mínima entropia, apresentado na subseção 4.1.1, para selecionar as estatísticas resumo para algoritmo $D$. No caso do fGn as estatísticas 1. a 4. nunca são escolhidas. As estatísticas baseadas na variação quadrática com filtro de diferença finita de ordem 1 a 5 , e suas transformações, são as únicas selecionadas. Essa escolha fornece os melhores resultados tornando o método $\mathrm{ABC}$ baseado no algoritmo $\mathrm{D}$ um método preciso para a estimação pontual do índice de memória como veremos a seguir.

Consideramos o uso do método ABC semiautomático, apresentado na subseção 4.1.3, utilizando as estatísticas de variação quadrática do item 5 . e a função identidade para a função $h(\cdot)$ deste método. Neste caso os resultados obtidos também são satisfatórios.

A Figura 5.1 apresenta os boxplots dos desvios relativos com respeito ao valor nominal de $H$ quando este varia em $\{0.2,0.5,0.7,0.9\}$. Quanto mais concentrado em torno de zero o desvio está, mais preciso é o método de estimação. O sumário desses resultados são dados na Tabela A.1. Para cada valor nominal de $H$ temos 10 boxplots onde cada um corresponde a um método de estimação a saber:

1. Análise do variograma;

2. análise $\mathrm{R} / \mathrm{S}$; 
3. método do periodograma;

4. análise DF;

5. método do periodograma global (FEXP);

6. método FELW;

7. método de ondaletas;

8. máxima verossimilhança (Whittle);

9. $\mathrm{ABC}$ (algoritmo D);

10. ABC semiautomático (SAABC).

Observe que os métodos $\mathrm{ABC}$ propostos apresentam resultados melhores que os obtidos por máxima verossimilhança através do método de Whittle, com exceção do caso $H=0.2$ quando se utiliza o método semiautomático.

Vale observar que com o aumento do comprimento da série observada, as estimativas obtidas pelos métodos clássicos concentram-se mais em torno do valor nominal do parâmetro, especialmente para os métodos 5. a 8., que são estimadores consistentes para o índice de memória. No entanto, mesmo tomando séries de tamanho 10000 as estimativas obtidas pelos métodos ABC continuam melhores e as estimativas obtidas por máxima verossimilhança retornam resultados praticamente idênticos.

\subsubsection{Aplicação: Níveis mínimos anuais do rio Nilo}

A Figura 5.2 apresenta os níveis mínimos anuais (em centímetros) do rio Nilo medidos entre os anos 622 e 1284 em uma região próxima ao Cairo. Este conjunto de dados pode ser obtido em Beran (1994) ou através do pacote longmemo do software R. Este conjunto de dados possui um importante valor histórico, pois sua análise contribuiu para a descoberta do efeito Hurst (Hurst, 1951) e o posterior desenvolvimento de processos com memória longa.

Historicamente, os dados do rio Nilo são considerados Gaussianos e esta suposição parece ser razoável quando observamos o gráfico que compara os quantís empíricos da série observada com os quantís teóricos da distribuição normal (Figura 5.3(a)). Além disso, o histograma da série em conjunto com a curva da densidade da distribuição normal ajustada por máxima verossimilhança (Figura 5.3(b)) reforça essa suposição. Realizamos também 100 simulações de amostras normais com parâmetros iguais aos estimados por máxima verossimilhança da série observada e realizamos o teste de Kolmogorov-Smirnov comparando se a série observada e a simulada possuem a mesma distribuição. Apenas 7\% dos testes rejeitaram a hipótese de normalidade ao nível de $5 \%$.

Se observarmos o gráfico da série do rio Nilo, notamos o comportamento típico de uma série com memória longa, pois embora a série como um todo pareça estacionária, ela possui períodos onde permanece abaixo ou acima da média amostral (efeito Hurst).

A presença de memória longa nos dados é imediatamente confirmada pelas análises heurísticas. A Figura 5.3(c) apresenta a análise $\mathrm{R} / \mathrm{S}$ da série indicando que $H>0.5$, enquanto o resultado das análises do variograma, periodograma e DF são dadas na Tabela 5.1.

O fGn é muito utilizado como modelo para dados hidrológicos que sugerem um comportamento Gaussiano (Beran, 1994). Por essa razão, os estimadores clássicos funcionam muito bem, já que a hipótese de normalidade garante a consistência desses estimadores, especialmente do estimador de Whittle, como vimos no estudo de simulação.

Para testar nossa abordagem baseada em ABC, aplicamos o método baseado no algoritmo D e o método semiautomático (SAABC) utilizados no estudo de simulação, assumindo que os dados foram gerados pelo fGn. A priori para $H$ é uniforme no intervalo $(0,1)$. As estimativas pontuais obtidas por esses dois métodos (média aproximada a posteriori) e pelos demais métodos clássicos utilizados no estudo de simulação são dadas na Tabela 5.1. Note que as estimativas obtidas pelos 
fGn: $H=0.2$

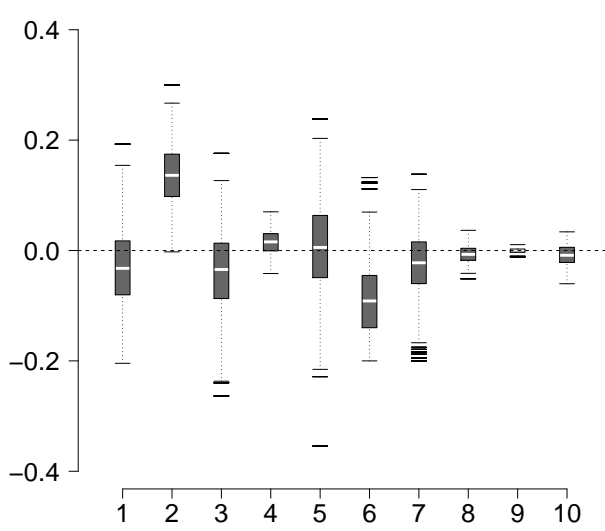

fGn: $H=0.7$

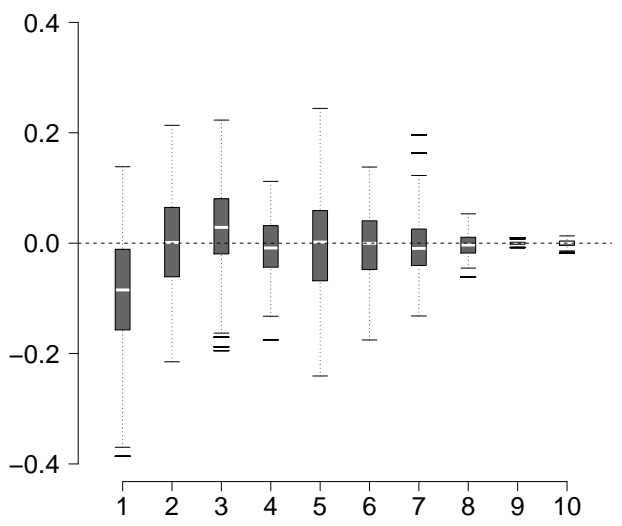

fGn: $H=0.5$

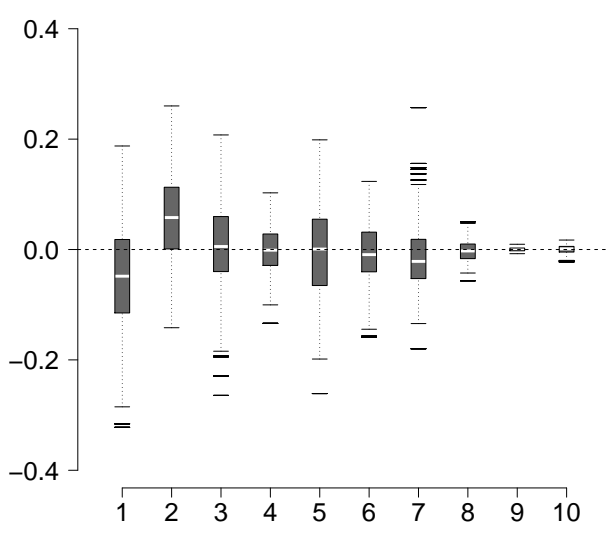

fGn: $H=0.9$

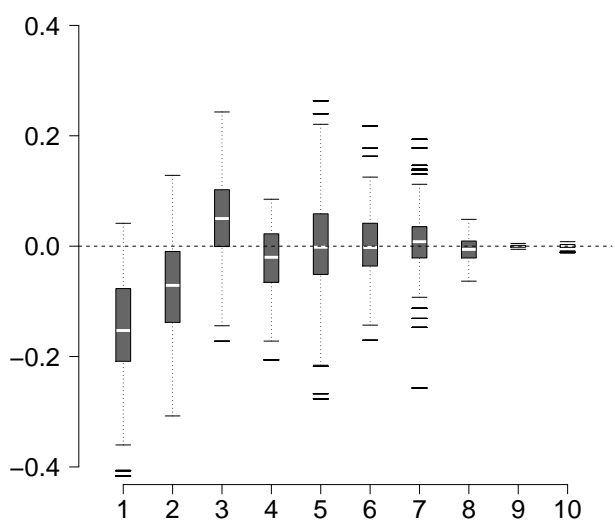

Figura 5.1: Boxplots dos desvios relativos com respeito ao valor nominal de $H \in\{0.2,0.5,0.7,0.9\}$ em 200 simulações do processo fGn segundo cada método de estimação. Cada número no eixo horizontal corresponde ao respectivo método de estimação enumerado anteriormente.

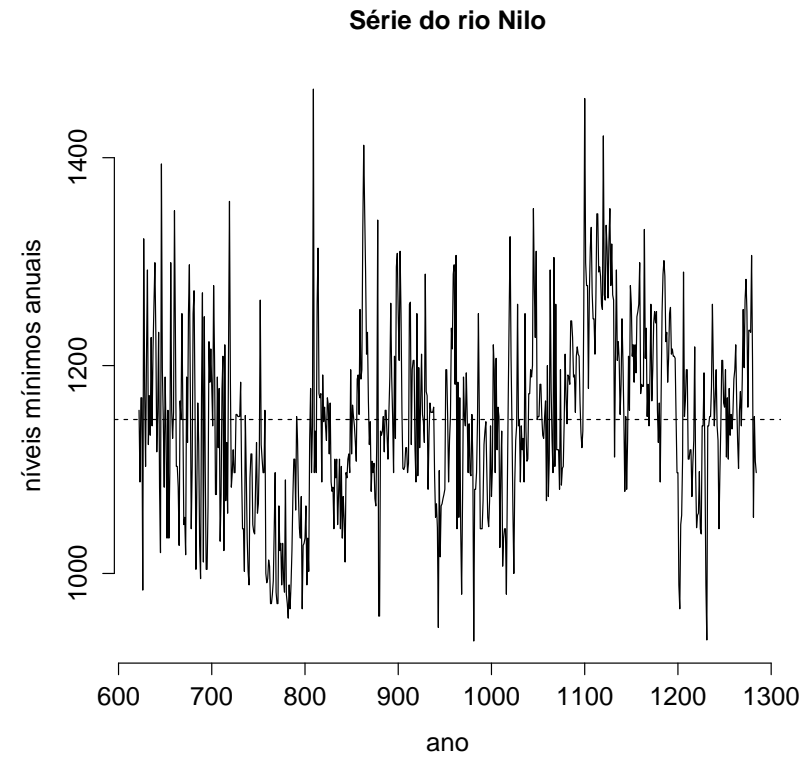

Figura 5.2: Niveis minimos anuais do rio Nilo entre os anos 622 e 1284. 


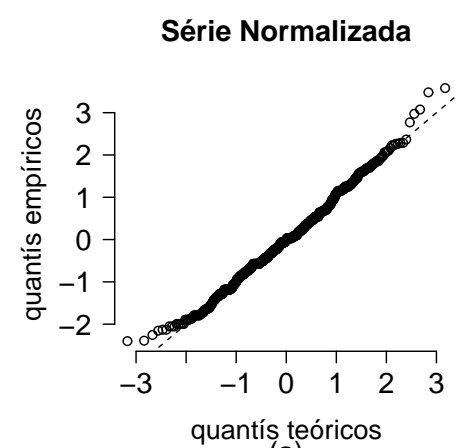

(a)

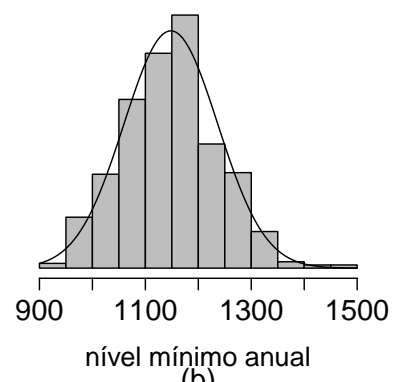

(b)

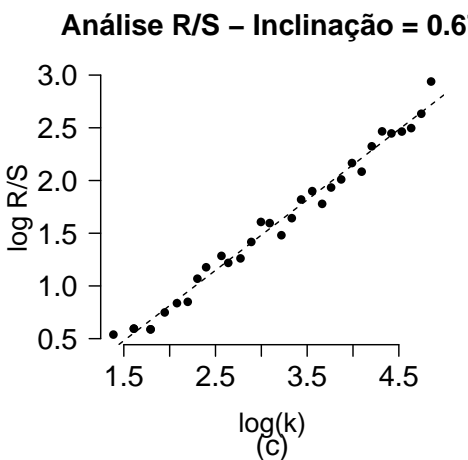

Figura 5.3: (a) Gráfico dos quantís teóricos da distribuição normal padrão contra os quantís empíricos da série observada padronizada. (b) Histograma da série observada e curva da densidade normal ajustada aos dados por máxima verossimilhança. (c) Análise $R / S$ da série observada.

métodos ABC estão de acordo com as abordagens clássicas, especialmente quando comparadas com o estimador de Whittle, o mais preciso dentre os estimadores clássicos utilizados sob a hipótese de normalidade (lembrando que este converge para o estimador de máxima verossimilhança).

O intervalo de confiança para $H$ pode ser obtido analiticamente para o fGn (Beran, 1994), neste caso o intervalo de $95 \%$ de confiança para $H$ é dado por $(0.7864,0.8884)$. Alternativamente, podemos obter um intervalo de credibilidade aproximado baseado nas amostras obtidas pelos métodos ABC. Os intervalos com 95\% de probabilidade obtidos pelo algoritmo D e pelo SAABC foram, respectivamente, $(0.8044,0.9084)$ e $(0.7805,0.8927)$.

Como utilizamos priori uniforme para $H$, temos que a distribuição a posteriori deveria ser proporcional a verossimilhança restrita ao intervalo $(0,1)$. Como o estimador de Whittle converge para o estimador de máxima verossimilhança sob o modelo fGn e os resultados obtidos por ABC são praticamente idênticos aos resultados obtidos pelo método de Whittle, acreditamos que a nossa abordagem aproxima adequadamente a verdadeira distribuição a posteriori. A Figura 5.4 apresenta as densidades aproximadas a posteriori obtidas pelo algoritmo D e pelo SAABC.

\subsection{Incrementos do processo de Rosenblatt}

Nesta seção reportamos os resultados obtidos por ABC para os incrementos do processo de Rosenblatt (que denotaremos por Rn) e comparamos com os resultados obtidos pelos métodos clássicos. Para esse processo, temos que $\theta=H \operatorname{com} H \in\left(\frac{1}{2}, 1\right)$.

Neste caso, vimos que o processo não é Gaussiano e os métodos de estimação clássicos, embora aplicáveis, passam a ser influênciados por esse desvio da normalidade. A consistência de estimadores como FELW, ondaleta e Whittle continua válida, mas a velocidade de convergência para o valor nominal passa a ser menor e controlada pelo valor de $H$.

Estudamos o mesmo conjunto de estatísticas utilizadas na seção anterior e notamos um desempenho similar das estatísticas de variação quadrática em conjunto com o algoritmo D. Desta vez as estatísticas mais selecionadas através de mínima entropia foram aquelas baseadas em filtros de 


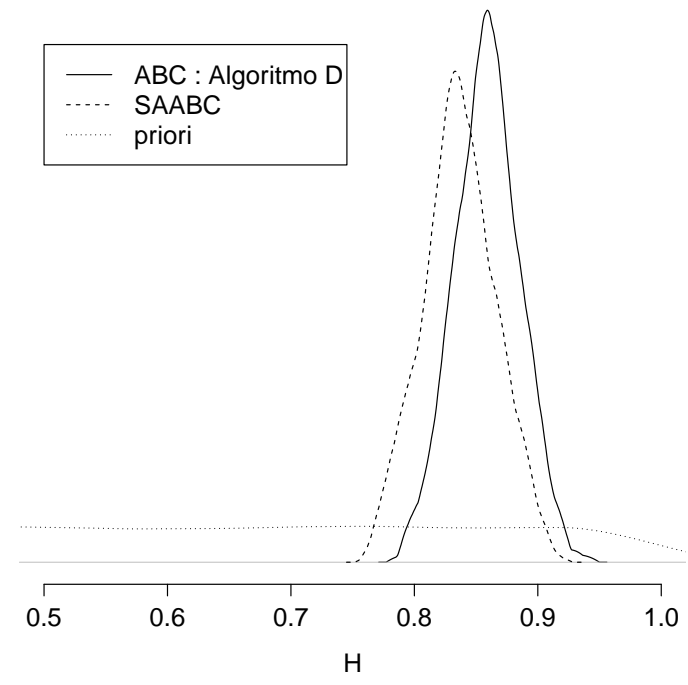

Figura 5.4: Posterioris aproximadas para $H$ : (linha cheia) obtida pelo algoritmo $D$ e (linha tracejada) ABC semiautomático. A linha pontilhada indica a distribuição a priori.

\begin{tabular}{lc}
\hline \hline Método de estimação & $\hat{H}$ \\
\hline Variograma & 0.8467 \\
R/S & 0.6677 \\
Periodograma & 0.9927 \\
DFA & 0.8268 \\
FEXP & 1.0018 \\
FELW & 0.9076 \\
Ondaleta & 0.8695 \\
Whittle & 0.8374 \\
ABC & 0.8590 \\
SAABC & 0.8366 \\
\hline \hline
\end{tabular}

Tabela 5.1: Estimativas de $H$ para a série de níveis minimos anuais do rio Nilo obtidas por diferentes métodos.

diferença finita de ordem 3 e 4 . Naturalmente, a priori utilizada é uniforme no intervalo $\left(\frac{1}{2}, 1\right)$. O uso de pós-processamento da amostra obtida pelo algoritmo D não influencia os resultados de forma significativa.

Diferente do que ocorre com o fGn, a forma da distribuição marginal do Rn muda de acordo com o valor de $H$. Por esta razão, as estatísticas de ordem fornecem alguma informação com respeito ao parâmetro de memória. Logo, implementamos também o algoritmo ABC semiautomático utilizando as estatísticas de ordem e suas potências de ordem 2 a 4 . Os resultados obtidos foram tão bons quantos os obtidos pelo algoritmo D.

Notamos um bom desempenho dos métodos $\mathrm{ABC}$ quando comparado com seus competidores clássicos, visto que os resultados obtidos por $\mathrm{ABC}$ competem diretamente com as estimativas de Whittle. Os boxplots dos desvios relativos com respeito ao valor nominal de $H$ obtidas segundo cada método encontram-se na Figura 5.5. Neste caso, variamos $H$ em $\{0.55,0.75,0.85,0.95\}$. O sumário desses resultados são dados na Tabela A.2. 
$\mathrm{Rn}: \mathrm{H}=\mathbf{0 . 5 5}$

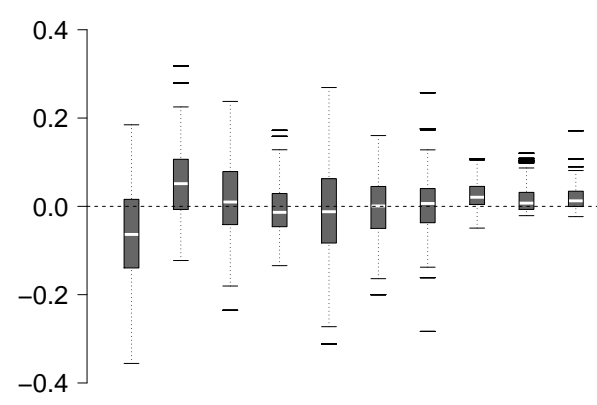

\begin{tabular}{lllllllllll}
\hline & 2 & 3 & 4 & 5 & 6 & 7 & 8 & 9 & 10
\end{tabular}

Rn: $\mathbf{H}=0.85$

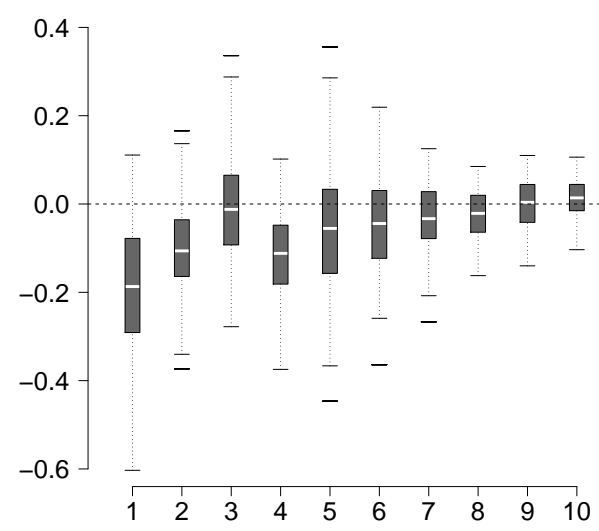

Rn: $\mathbf{H}=\mathbf{0 . 7 5}$

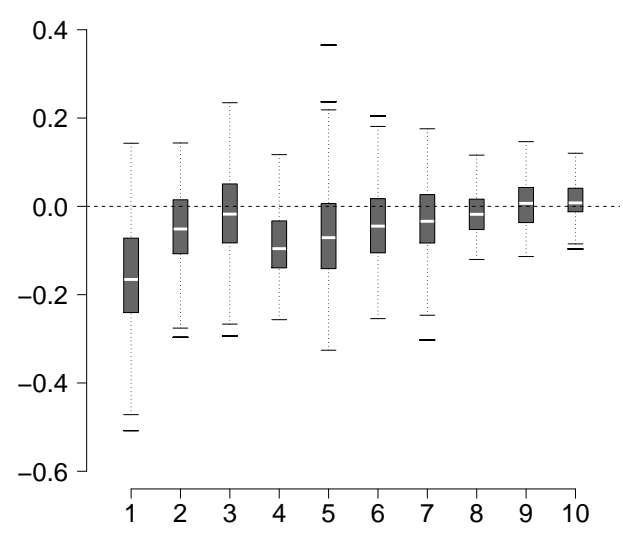

Rn: $\mathbf{H}=\mathbf{0 . 9 5}$

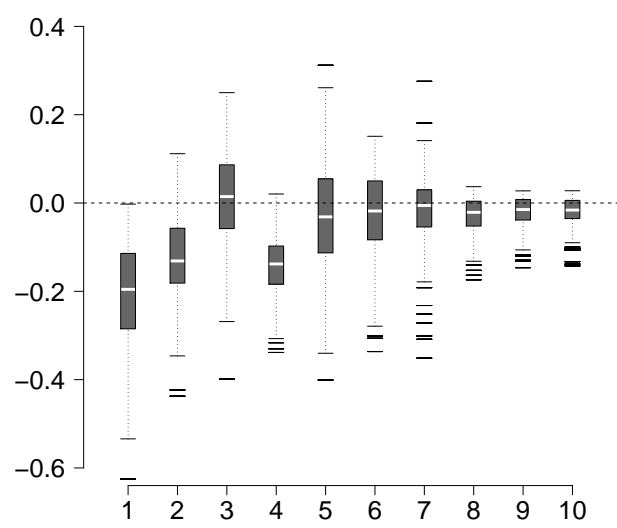

Figura 5.5: Boxplots dos desvios relativos com respeito ao valor nominal de $H \in\{0.55,0.75,0.85,0.95\}$ em 200 simulações do processo Rn segundo cada método de estimação. Cada número no eixo horizontal corresponde ao respectivo método de estimação enumerado anteriormente.

\subsubsection{Aplicação: Vazão média mensal dos rios Mosa e Vístula}

As Figuras 5.6(a,b) apresentam as séries da vazão média mensal $\left(\mathrm{em} \mathrm{m}^{3} / \mathrm{s}\right)$ de dois rios Europeus:

- Rio Mosa (ou Mass) monitorado entre os anos 1911 e 1983 pela estação Lith na Holanda.

- Rio Vístula (ou Wisla) monitorado entre os anos 1901 e 1985 pela estação Tczew na Polônia.

Os dados foram obtidos através do site do Center of Sustainability and Global Environment ${ }^{1}$. A simulação estocástica da vazão de rios é importante para estabelecer medidas de gerenciamento dos recursos hídricos e, como comentamos no início da seção 2.2, negligenciar a presença de memória longa nos dados pode levar a uma subestimação de intervalos de previsão.

Antes de realizarmos qualquer análise sobre a estrutura de dependência, é importante mencionar que dados hidrológicos mensais possuem uma forte componente sazonal causada especialmente por efeitos climáticos. Em geral esta componente é removida antes de se aplicar qualquer método inferencial. Nesta análise iremos remover a sazonalidade utilizando o método STL (seasonal-trend decomposition based on loess) de Cleveland et al. (1990). Os dados livres de sazonalidade podem ser visualizados nas Figuras 5.7(a) e 5.8(a). A partir de agora iremos aplicar os métodos de estimação nessas novas séries.

\footnotetext{
${ }^{1}$ https://nelson.wisc.edu/sage/data-and-models/riverdata/index.php
} 
(a) - Série original do rio Mosa

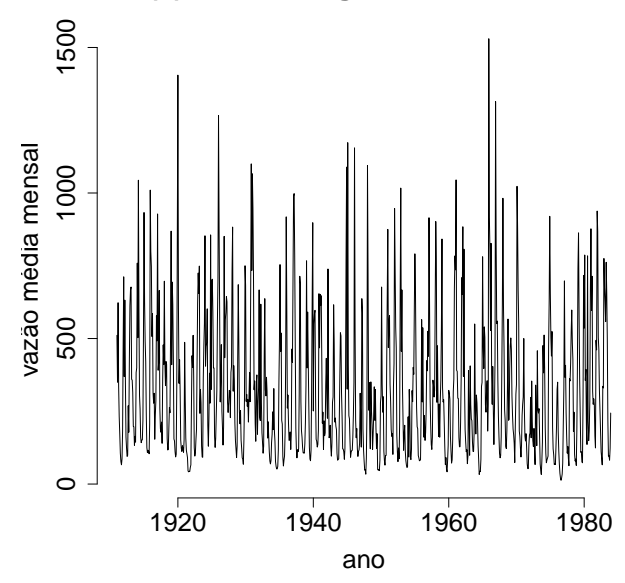

(b) - Série original do rio Vístula

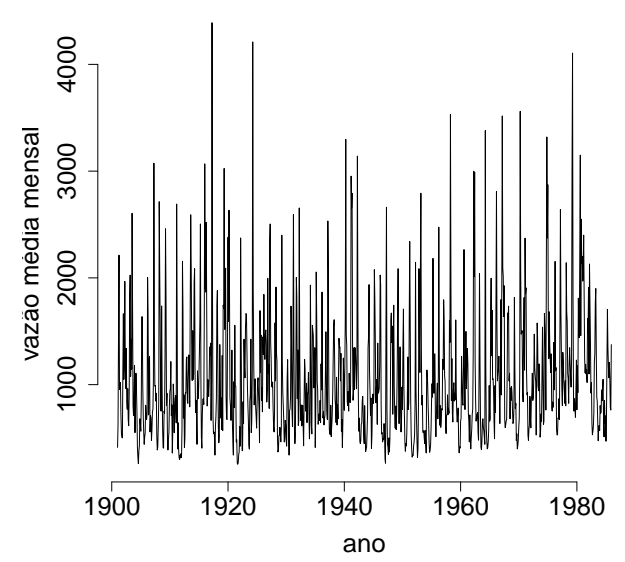

Figura 5.6: Vazão média mensal dos rios Mosa e Vístula.

Observe que os gráficos de quantís (Figuras 5.7(b) e 5.8(b)) e os histogramas (Figuras 5.7(c) e $5.8(\mathrm{c}))$ das séries livres de sazonalidade indicam que os dados possuem assimetria positiva. Assim como fizemos para os dados do rio Nilo, simulamos 100 amostras normais com parâmetros estimados via máxima verossimilhança de cada série e realizamos testes de Kolmogorov-Smirnov para testar se as séries observadas e simuladas possuem a mesma distribuição. Para ambas as séries, a normalidade é rejeitada ao nível de $5 \%$ em $100 \%$ das simulações.

Assim como observado nos dados do rio Nilo, as séries livres de sazonalidade apresentam o efeito Hurst. Além disso, uma análise heurística preliminar sugere que ambas as séries possuem memória longa. Os gráficos da análise R/S de cada série são dadas nas Figuras 5.7(d) e 5.8(d).

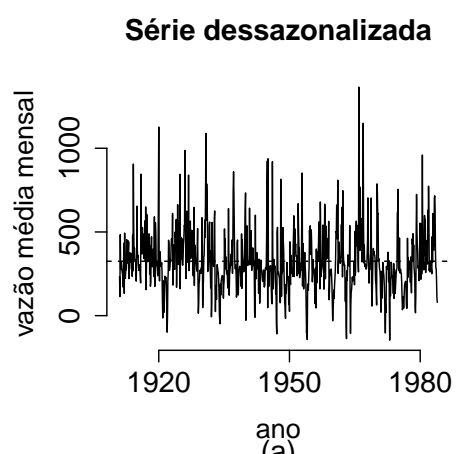

(a)

Série dessazonalizada

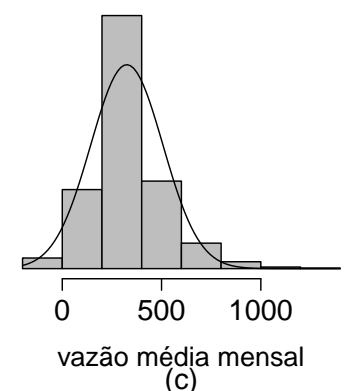

Série Normalizada

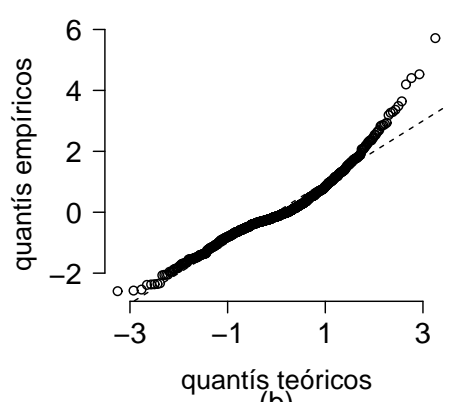

(b)

Análise R/S - Inclinação = 0.76

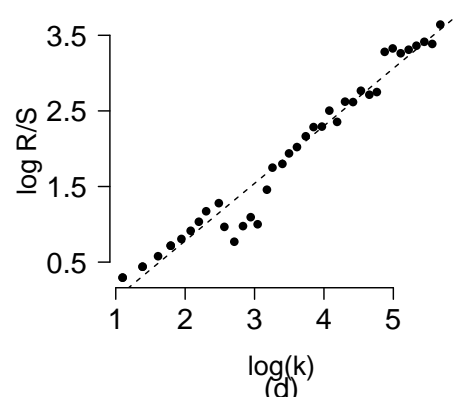

Figura 5.7: (a) Série do rio Mosa livre de sazonalidade. (b) Gráfico dos quantís teóricos da distribuição normal padrão contra os quantís empíricos da série observada padronizada. (c) Histograma da série observada (dessazonalizada) e curva da densidade normal ajustada aos dados por máxima verossimilhança. (d) Análise $R / S$ da série observada (dessazonalizada). 


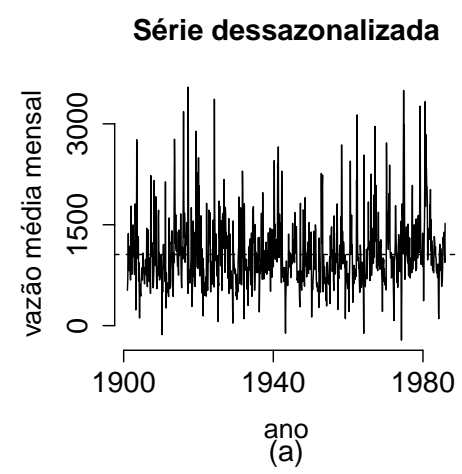

Série dessazonalizada

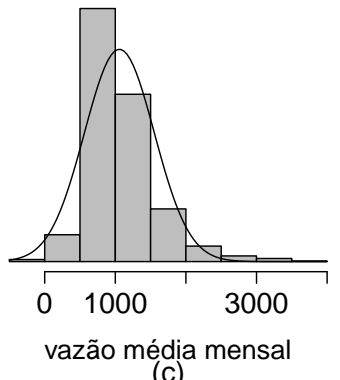

Série Normalizada

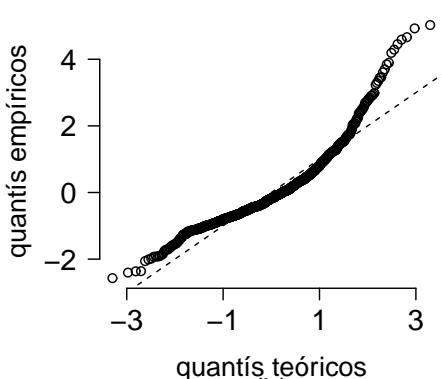

(b)

Análise R/S - Inclinação = 0.75

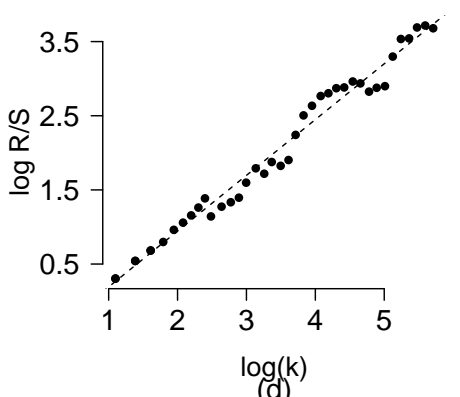

Figura 5.8: (a) Série do rio Vistula livre de sazonalidade. (b) Gráfico dos quantís teóricos da distribuição normal padrão contra os quantís empíricos da série observada padronizada. (c) Histograma da série observada (dessazonalizada) e curva da densidade normal ajustada aos dados por máxima verossimilhança. (d) Análise $R / S$ da série observada (dessazonalizada).

A distribuição marginal dos incrementos do processo de Rosenblatt ( $\mathrm{Rn}$ ) possui assimetria positiva. Por essa razão optamos por utilizar este processo como modelo para as séries observadas ao invés de utilizar o fGn. A priori utilizada agora é uniforme no intervalo $(0.5,1)$, pois o processo de Rosenblatt não está definido para $H<0.5$. Vamos utilizar novamente o método ABC baseado no algoritmo $\mathrm{D}$, o método $\mathrm{ABC}$ semiautomático (SAABC) e todos os métodos de estimação empregados no estudo de simulação. Os resultados são apresentados na Tabela 5.2.

Observe que os resultados obtidos pelos métodos ABC estão novamente de acordo com os seus competidores clássicos. Embora a suposição de normalidade não seja razoável, o estimador de Whittle ainda é consistente para o parâmetro de memória quando o modelo é o Rn, como demonstrado recentemente em Bardet e Tudor (2014). Neste caso, para $H>0.75$ a distribuição assintótica do estimador é dado em termos da distribuição de Rosenblatt apresentada no Exemplo 9. Além disso, a taxa de convergência deixa de ser $\sqrt{n}$, onde $n$ é o tamanho da amostra, e passa a ser $n^{1-H}$, tornando os intervalos de confiança obtidos sob o modelo Rn mais esparso que no caso Gaussiano. Os intervalos de $95 \%$ de confiança para $H$ obtidos neste caso são dados por $(0.6730,0.8685)$ para os dados do rio Mosa e $(0.6787,0.8928)$ para os dados do rio Vístula. Os intervalos aproximados de credibilidade de $95 \%$ para $H$ obtidos pelo $\mathrm{ABC}$ baseado no algoritmo $\mathrm{D}$ foram, respectivamente, $(0.6788,0.9166)$ e $(0.7257,0.9391)$ para os dados dos rios Mosa e Vístula. Para o método SAABC os intervalos aproximados foram respectivamente iguais a $(0.6880,0.9317)$ e $(0.7068,0.9477)$. Assim como os intervalos clássicos, os intervalos Bayesianos aumentaram em amplitude se comparados com a amplitude dos intervalos obtidos para o índice de memória do rio Nilo, onde o modelo fGn foi utilizado. Novamente, o fato de termos utilizado priori uniforme e os resultados do ABC ficarem próximos dos resultados obtidos pelo método de Whittle, acreditamos que a nossa abordagem aproximou adequadamente a posteriori de $H$. A Figura 5.9 apresenta essas posterioris.

A distribuição marginal do processo de Rosenblatt é dada pela distribuição de Rosenblatt com parâmetro $D=2-2 H$ (Veja o Exemplo 9). A Figura 5.10 apresenta novamente os histogramas das séries livres de sazonalidade padronizadas de cada rio e a curva da densidade da distribuição de 
(a) Rio Mosa

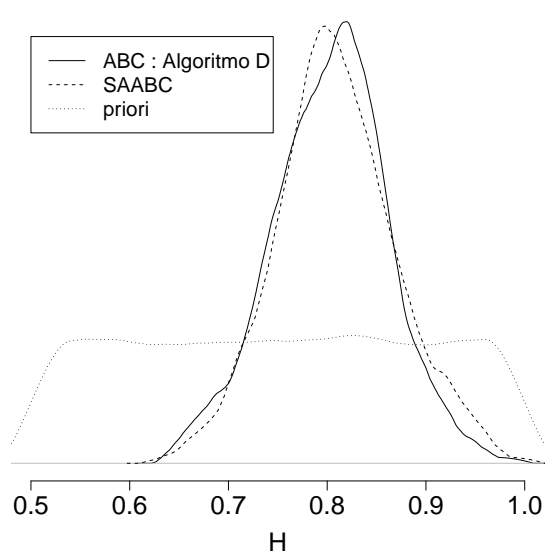

(b) Rio Vístula

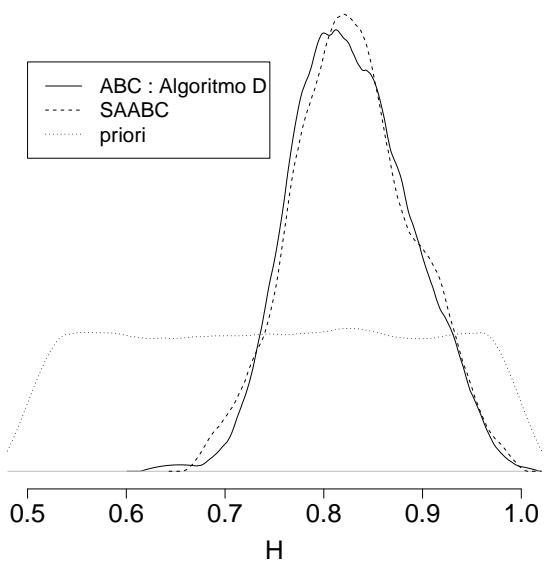

Figura 5.9: Posterioris aproximadas para o parâmetro $H$ obtidas pelos métodos ABC para os dados dos rios Mosa e Vistula.

Rosenblatt com parâmetro $\hat{D}=2-2 \hat{H}$, onde $\hat{H}$ são as estimativas obtidas pelo método SAABC. Essas curvas foram obtidas numericamente utilizando as técnicas apresentadas em Veillette et al. (2013). Observe que as curvas se ajustam melhor nos dados do que a curva normal.

(a)

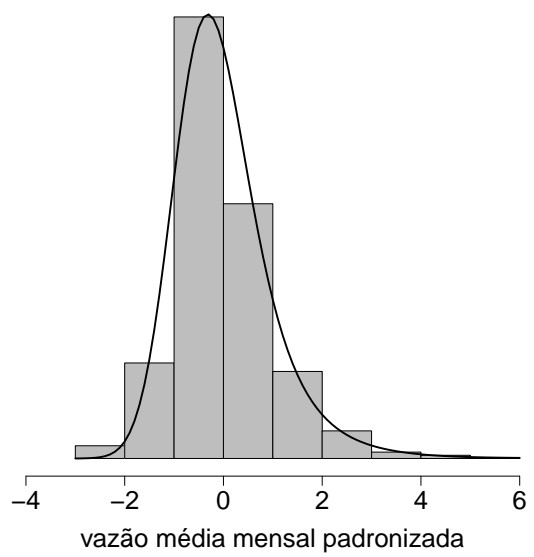

(b)

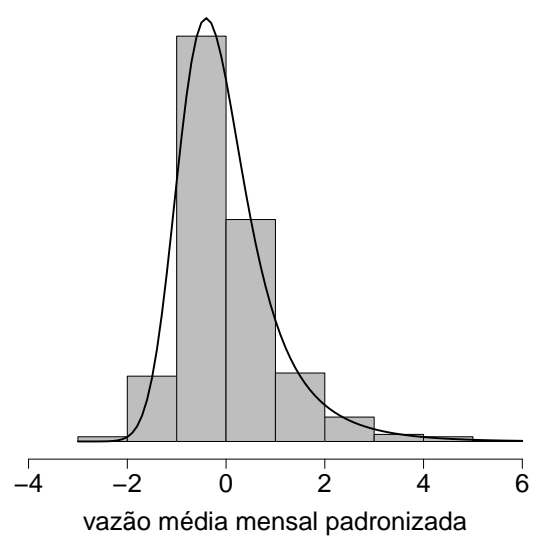

Figura 5.10: Histograma das séries dessazonalizadas e padronizadas dos rios Mosa (a) e Vistula (b) com curva da densidade da distribuição de Rosenblatt com parâmetro $\hat{D}=2-2 \hat{H}$, onde $\hat{H}$ são, respectivamente iguais a 0.8089 e 0.8297 .

\subsection{Processo binário com memória longa}

Esta aplicação, apresentada em Andrade e Rifo (2015b), nos motivou a utilizar métodos ABC para estimar o índice de memória em processos mais gerais. Neste caso, um processo binário assumindo valores em $\{0,1\}$ é construído artificialmente a partir de um ruído Gaussiano fracionário, de modo que a estrutura de dependência de longo alcance seja preservada, mas a natureza Gaussiana dos dados é perdida. Assim, os métodos usuais passam a falhar conforme o índice de memória se distancia do valor 0.5 .

Uma trajetória binária $y_{1}, \ldots, y_{n}$ com índice de memória $H$ é construída seguindo o algoritmo abaixo:

- Simule uma trajetória $x_{1}, \ldots, x_{n}$ do fGn padrão com parâmetro de memória $H$; 


\begin{tabular}{lcc}
\hline \hline Método de Estimação & Mosa & Vístula \\
\hline Variograma & 0.5975 & 0.6337 \\
R/S & 0.7605 & 0.7522 \\
Periodograma & 0.8911 & 0.8157 \\
DFA & 0.6757 & 0.6134 \\
FEXP & 0.6943 & 0.6357 \\
FELW & 0.7655 & 0.6845 \\
Ondaleta & 0.7379 & 0.7978 \\
Whittle & 0.7848 & 0.8032 \\
ABC & 0.8030 & 0.8285 \\
SAABC & 0.8089 & 0.8297 \\
\hline \hline
\end{tabular}

Tabela 5.2: Estimativas de $H$ para as séries de vazão média mensal dos rios Mosa e Vístula por diferentes métodos.

- para $i=1, \ldots, n$, calcule $p_{i}=\Phi\left(x_{i}\right)$, onde $\Phi$ denota a função de distribuição acumulada da distribuição normal padrão;

- para $i=1, \ldots, n$, obtenha os quantís $y_{i}$ com probabilidade $p_{i}$ da distribuição Bernoulli(1/2).

A Figura 5.11 nos mostra 3 trajetórias binárias simuladas pelo algoritmo acima com $H \in$ $\{0.5,0.7,0.9\}$. Note que conforme o índice de memória cresce a série tende a permanecer por mais tempo nos estados 0 ou 1.
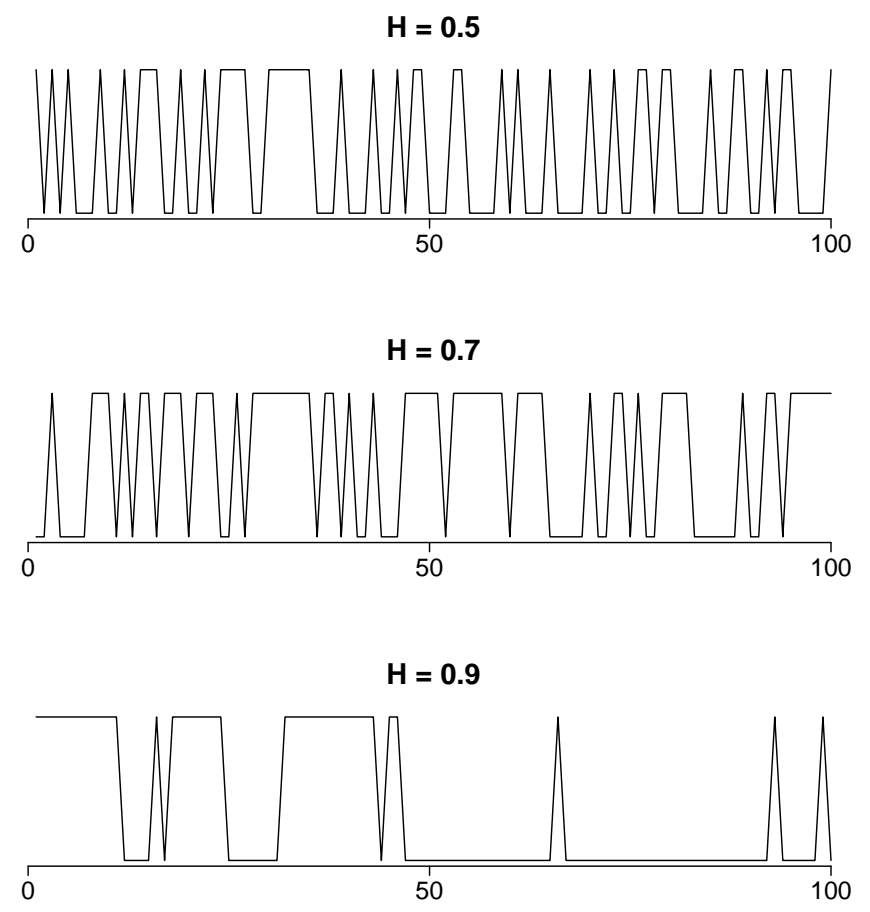

Figura 5.11: Trajetórias amostrais de comprimento 100 do processo binário com diferentes indices de memória.

Procedemos da mesma maneira que no caso fGn e os resultados são reportados na Figura 5.12. Neste caso, a análise DF não retorna resultados safisfatórios para nenhum valor de $H$ enquanto os demais competidores clássicos apresentam resultados razoáveis apenas no caso $H=0.5$, isto é, quando temos uma sequência i.i.d Bernoulli(1/2). Nos demais casos apenas as abordagens baseadas em métodos $\mathrm{ABC}$ retornam estimativas satisfatórias. 
É importante notar que esse estudo apenas ilustra como o ABC pode ser uma alternativa interessante desde que o modelo probabilístico escolhido para os dados seja adequado. Neste caso conhecemos o dispositivo gerador dos dados, ou seja, espera-se que o ABC tenha um desempenho melhor frente aos métodos clássicos que são mais gerais.

\section{$\mathrm{P} 0-1: \mathrm{H}=0.2$}

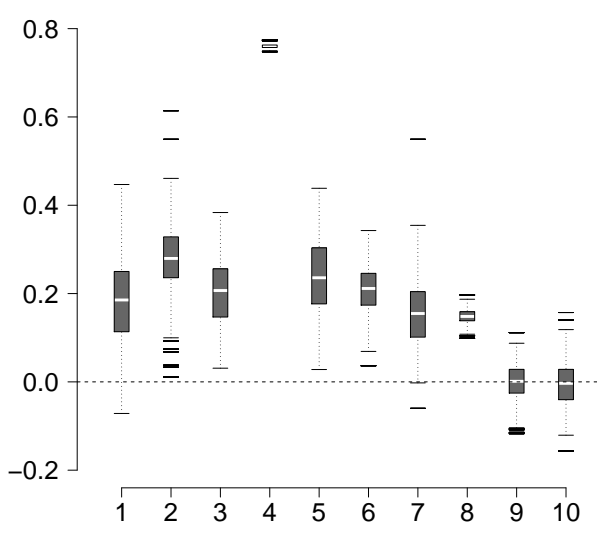

$\mathrm{P0}-1: \mathrm{H}=0.7$

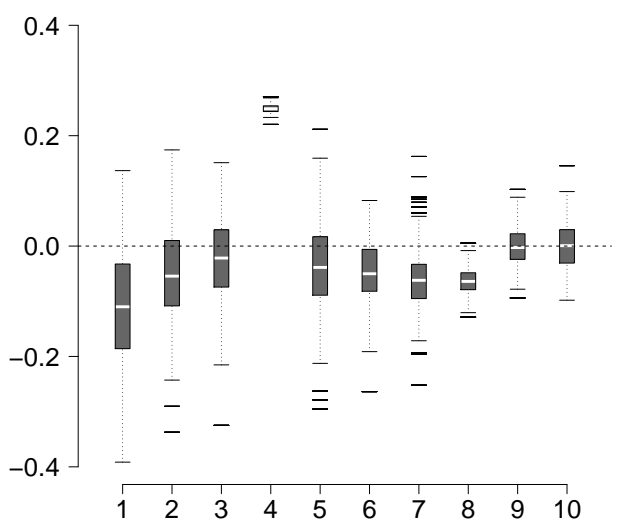

$\mathrm{P} 0-1: \mathrm{H}=0.5$

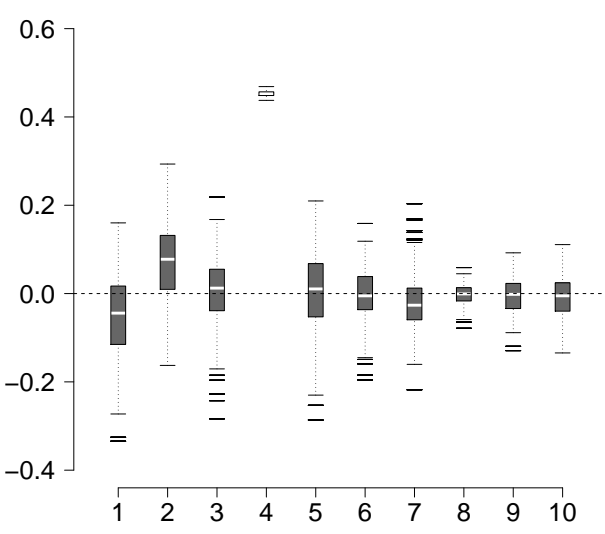

P0-1: $\mathrm{H}=0.9$

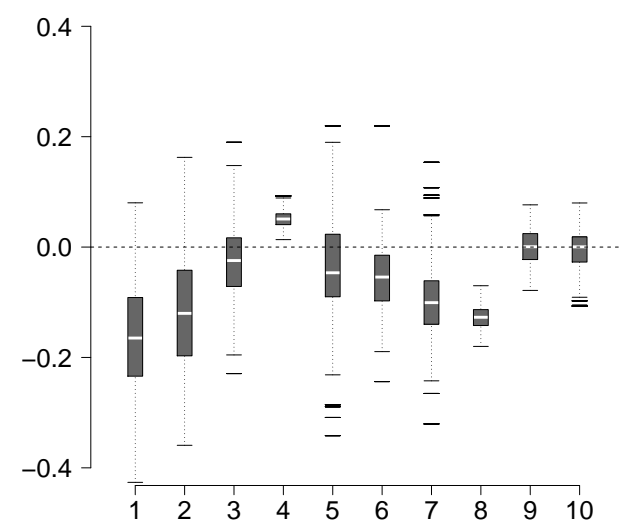

Figura 5.12: Boxplots dos desvios relativos com respeito ao valor nominal de $H \in\{0.2,0.5,0.7,0.9\}$ em 200 simulações do processo binário com memória longa segundo cada método de estimação. Cada número no eixo horizontal corresponde ao respectivo método de estimação enumerado anteriormente.

\subsection{Movimento estável fracionário}

Nesta seção iremos estudar o ruído estável fracionário (fSn) apresentado na seção 2.2.3. Neste caso, dois parâmetros deverão ser estimados, a saber, o parâmetro de estabilidade $\alpha$ e o índice de memória $H$. Logo, $\theta=(\alpha, H)$ e a memória do processo é dada em termos de $d=H-\frac{1}{\alpha}$.

Vamos estudar os casos onde há memória longa, isto é, quando $d>0\left(H>\frac{1}{\alpha}\right)$. Para isso, devemos ter $\alpha \in(1,2)$ e $H \in\left(\frac{1}{2}, 1\right)$ com $H>\frac{1}{\alpha}$. Recorde que quando $\alpha=2$ o fSn se reduz ao fGn e quando $\alpha<2$ a distribuição marginal do fSn possui variância infinita.

Embora o fSn não satisfaça condições de segunda ordem, uma série observada de tal processo possui variância finita, de modo que métodos de estimação como a análise do variograma, $\mathrm{R} / \mathrm{S}$ entre outras podem ser aplicadas, porém os resultados obtidos não são satisfatórios por serem viesados e possuírem grande variabilidade. O método de ondaletas e o método FELW, utilizados nos estudos anteriores, estimam a memória do fSn consistentemente (ambos são semiparamétricos), especialmente o FELW, que foi desenvolvido com o intuito de ser aplicado em processos não Gaussianos mais gerais como o movimento estável fracionário. No caso do fGn, esses estimadores retornavam 
uma estimativa de $d=H-\frac{1}{2}$, mas no caso do fSn, estes retornam estimativas de $d=H-\frac{1}{\alpha}$. Vamos comparar os resultados obtidos pelos métodos $\mathrm{ABC}$ com os seguintes métodos:

1. Variação- $p$, que denotaremos por var- $p$;

2. método MSD;

3. método FELW;

4. método de ondaletas, que denotaremos por Wave.

O fSn é um processo estacionário cuja distribuição marginal é uma v.a. estável com parâmetro de estabilidade $\alpha$. Para obter informação sobre este parâmetro, ou estimá-lo, devemos considerar que a série observada do fSn é uma amostra aleatória de v.a.'s estáveis. Existem vários métodos clássicos de estimação para os parâmetros de uma distribuição estável. Os métodos mais utilizados são o da função característica empírica (ECF) de Koutrouvelis (1980) e o método dos percentís de McCulloch (1986). Este último, embora mais eficiente computacionalmente, é levemente menos preciso que o primeiro logo, para as abordagens clássicas, iremos utilizar o método de Koutrouvelis (1980) para estimar $\alpha$.

Recentemente, Peters et al. (2012) utilizaram um método ABC para estimar os parâmetros de distribuições estáveis considerando diversas estatísticas resumo, entre elas foram incluídas como estatísticas as estimativas fornecidas pelos métodos mencionados acima. Neste trabalho iremos utilizar como estatísticas resumo para $\alpha$ a estimativa obtida pelo método de Koutrouvelis (1980). Os resultados obtidos pelo $\mathrm{ABC}$ para $\alpha$, através dessas estatísticas resumo, são praticamente idênticos aos obtidos em Peters et al. (2012).

Para o parâmetro $H$, testamos as mesmas estatísticas utilizadas nos casos fGn e Rn, mas os resultados não foram satisfatórios. Os melhores resultados foram obtidos através do uso do método da variação- $p$ amostral apresentado na seção 3.7 e do deslocamento quadrático médio amostral apresentado na seção 3.8 .

Supondo a priori que $\alpha \sim U[1,2]$ e $H \sim U[0.5,1]$, implementamos o método ABC semiautomático de duas formas:

- ABC 1: As estatísticas resumo são dadas pela estimativa de $\alpha$ obtida pelo método de Koutrouvelis (1980) e o valor $1 / \hat{p}$ obtido através do método da variação- $p$.

- ABC 2: As estatísticas resumo são dadas pela mesma estimativa de $\alpha$ e a inclinação da reta obtida pelo método MSD.

Para ambos os métodos, a função $h(\cdot)$ do método ABC semiautomático é a função identidade. O algoritmo D poderia ser utilizados, mas notamos que o método semiautomático fornece resultados mais precisos. Simulamos 200 trajetórias amostrais do fSn para cada $H$ entre os valores $\{0.6,0.7,0.8,0.9\}$ e cada $\alpha$ em $\{1.7,1.8,1.9\}$. Neste caso, $d>0$ para qualquer combinação de $H$ e $\alpha$, isto é, as séries apresentam memória longa.

Os boxplots das Figuras 5.13, 5.14, 5.15 e 5.16 apresentam os resultados obtidos em termos dos desvios relativos ao valor nominal de $d=H-\frac{1}{\alpha}$, isto é, os valores $\hat{d}-d$. Os resultados obtidos separadamente para $\alpha$ e $H$ são reportados respectivamente nas tabelas A.4 e A.5.

Quando o valor de $H$ aproxima-se de 0.5 (caso sem memória), os métodos MSD, ABC 1 e ABC 2 apresentam resultados similares para todos os valores de $\alpha$, enquanto o método var- $p$ torna-se cada vez mais preciso quando $\alpha$ aproxima-se de 2 (caso normal). Conforme $H$ aumenta os melhores resultados são obtidos pelos métodos $\mathrm{ABC}$, enquanto os métodos var- $p$ e MSD ficam viesados para todos os valores de $\alpha$. Em todos os casos, os métodos Wave e FELW retornam resultados razoáveis, especialmente quando o valor de $\alpha$ cresce, mas a variabilidade de ambos é maior que a dos métodos ABC. 
Desvios do valor nominal

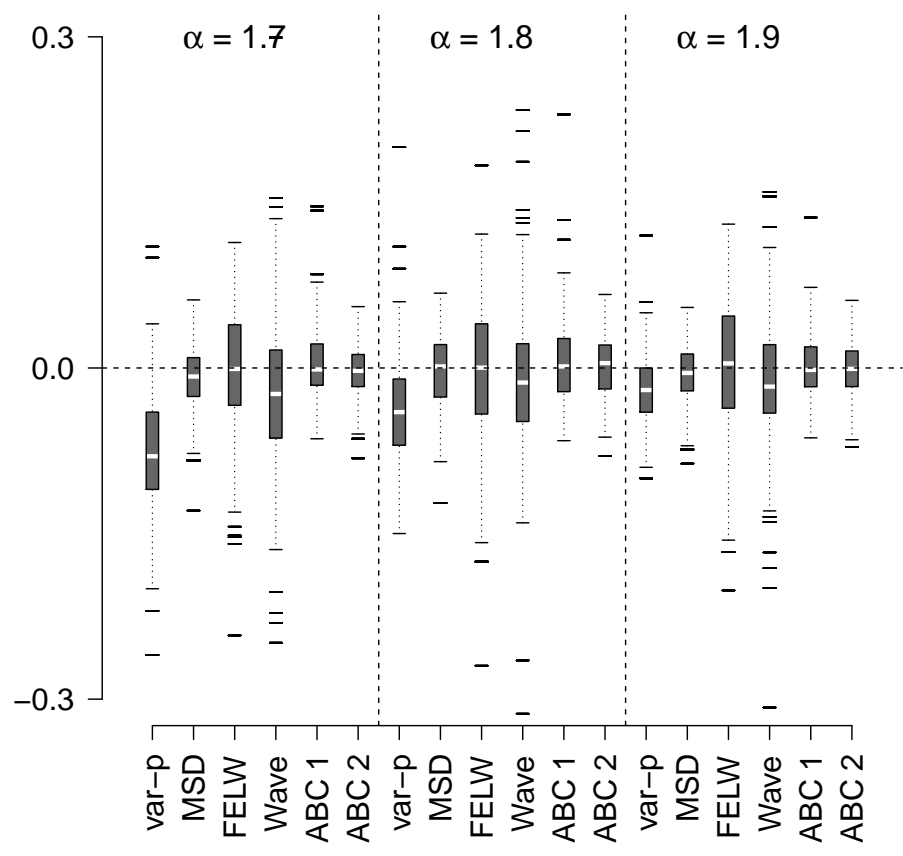

Figura 5.13: Boxplots dos desvios relativos com respeito ao valor nominal de $d=H-\frac{1}{\alpha}$, com $H=0.6$ $e \quad \alpha \in\{1.7,1.8,1.9\}$ (divididos pelas linhas verticais pontilhadas). Cada coluna corresponde a um método (etiquetado no eixo $0 x$ ).

\section{Desvios do valor nominal}

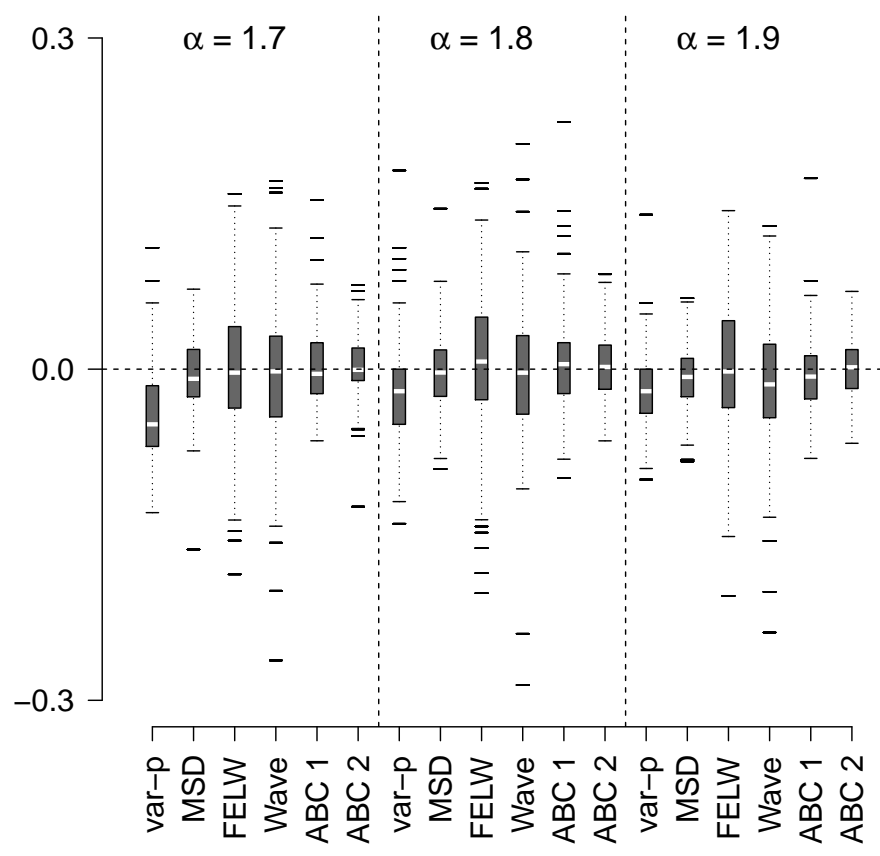

Figura 5.14: Boxplots dos desvios relativos com respeito ao valor nominal de $d=H-\frac{1}{\alpha}$, com $H=0.7$ $e \alpha \in\{1.7,1.8,1.9\}$ (divididos pelas linhas verticais pontilhadas). Cada coluna corresponde a um método (etiquetado no eixo $0 x$ ). 


\section{Desvios do valor nominal}

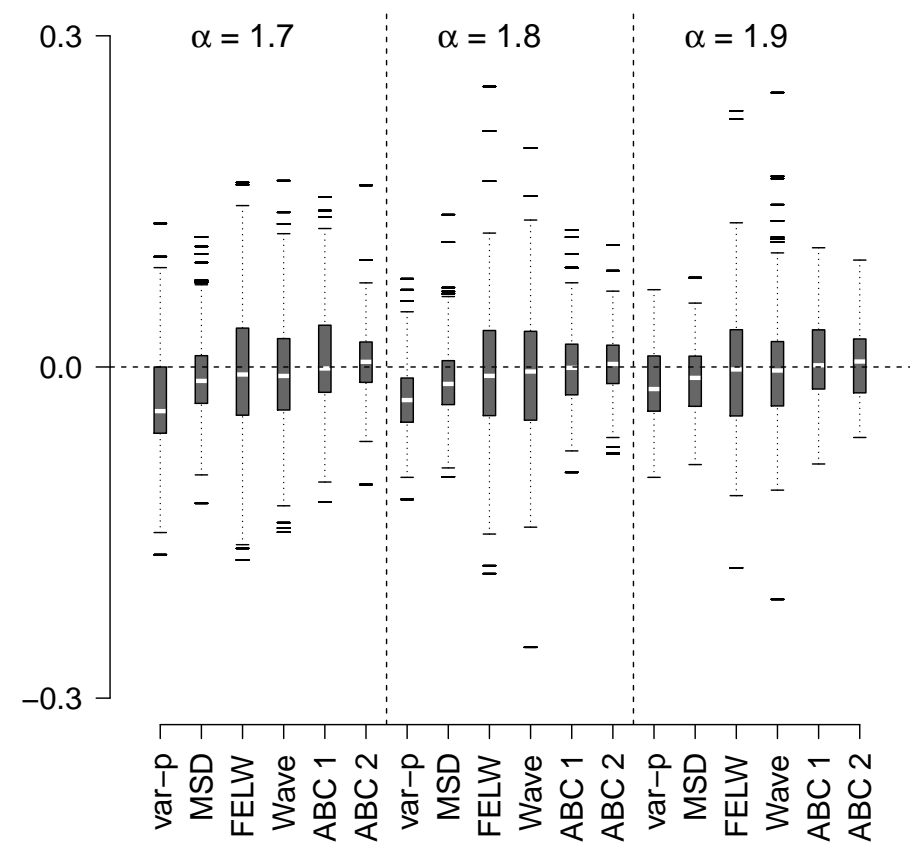

Figura 5.15: Boxplots dos desvios relativos com respeito ao valor nominal de $d=H-\frac{1}{\alpha}$, com $H=0.8$ e $\alpha \in\{1.7,1.8,1.9\}$ (divididos pelas linhas verticais pontilhadas). Cada coluna corresponde a um método (etiquetado no eixo $0 x$ ).

Desvios do valor nominal

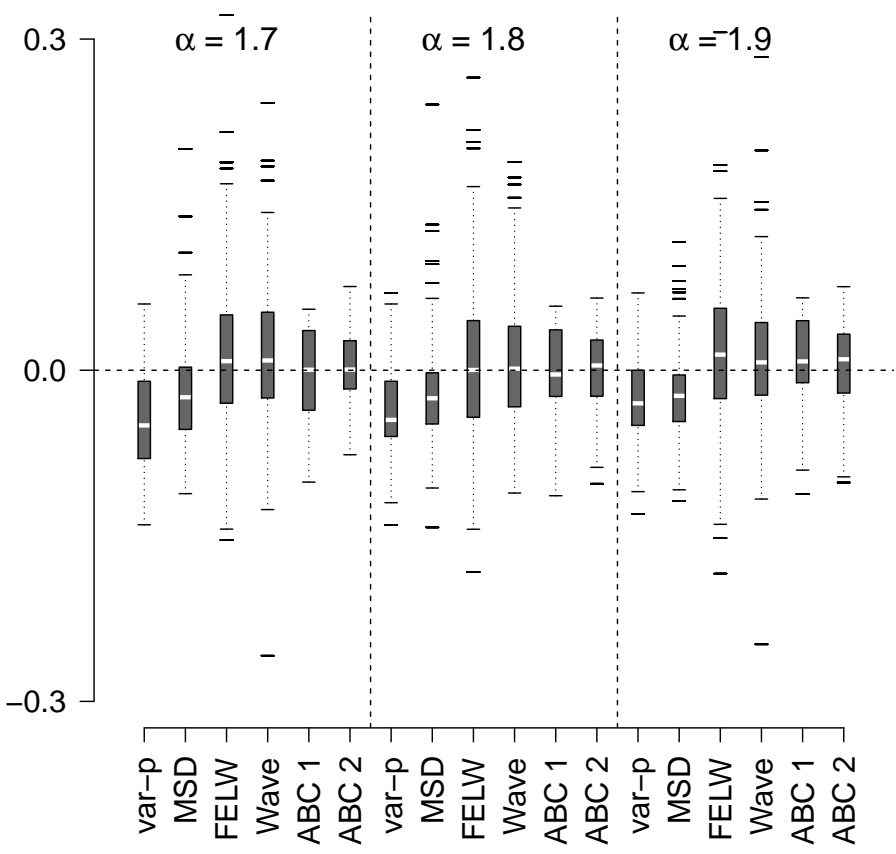

Figura 5.16: Boxplots dos desvios relativos com respeito ao valor nominal de $d=H-\frac{1}{\alpha}$, com $H=0.9$ e $\alpha \in\{1.7,1.8,1.9\}$ (divididos pelas linhas verticais pontilhadas). Cada coluna corresponde a um método (etiquetado no eixo $0 x$ ).

\subsubsection{Aplicação: Erupções solares}

Uma erupção solar é um aumento súbito de curta duração na intensidade de radiação emitida na superfície do sol na vizinhança de manchas solares. Essas erupções produzem radiações 
eletromagnéticas que no espaço podem causar sérios danos à equipamentos feitos pelo homem. A radiação que não é desviada pelo campo magnético terrestre pode causar interferências em canais de comunicação eletrônica e influenciar as flutuações da temperatura terrestre.

Emissões energéticas das erupções podem ser observadas com o auxílio de telescópios acoplados em satélites, pois essas emissões não são capazes de penetrar na atmosfera terrestre.

Vamos analisar um conjunto de dados de emissões energéticas obtidos pelo satélite GOES (Geostationary Operational Environmental Satellite). Esse conjunto de dados pode ser acessado no site do NGDC (National Geophysical Data Center ${ }^{2}$ ).

Os dados foram coletados diariamente no período de 1 de janeiro de 2003 a 31 de dezembro de 2005 e medem a energia em Watts por metro ao quadrado $\left(\mathrm{W} / \mathrm{m}^{2}\right)$. Esta série pode ser visualizada na Figura 5.17.

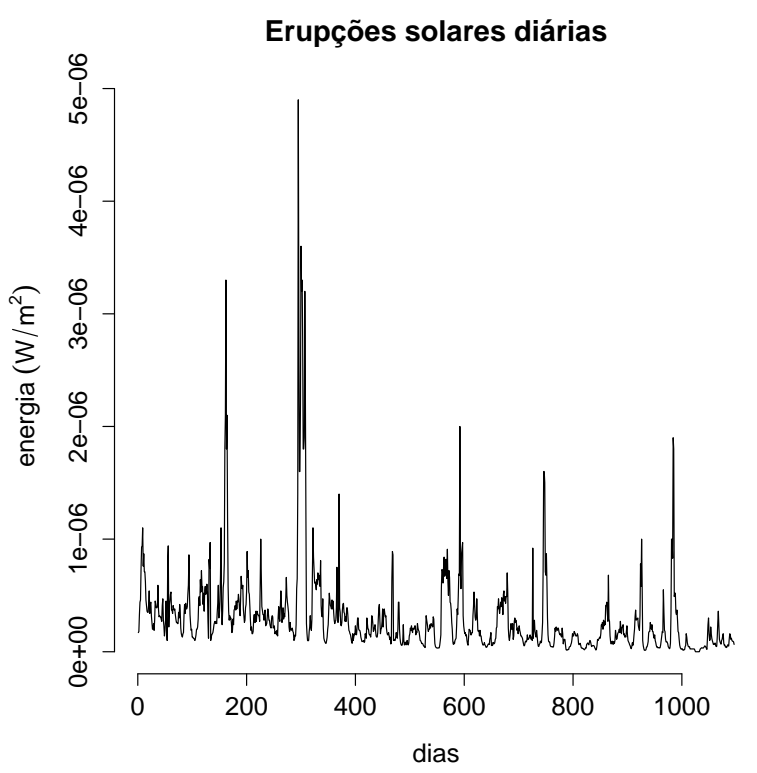

Figura 5.17: Emissões energéticas de erupções solares obtidas pelo satélite GOES no período de 2003 a 2005.

Burnecki et al. (2008) analisaram as emissões energéticas no período de 1999 a 2002 obtidas pelo satélite GOES, mas os dados não estavam mais disponíveis no site do NGDC. Os autores consideram que o processo que gerou os dados possuem memória longa com distribuição marginal estável e estimam os parâmetros de autossimilaridade $H$ e de estabilidade $\alpha$. Esses parâmetros podem ser utilizados para ajustar modelos como o fSn ou FARIMA (Beran, 1994; Doukhan et al., 2003) com inovações estáveis. Os dados analisados aqui apresentam as mesmas características dos dados utilizados em Burnecki et al. (2008) como veremos a seguir.

A Figura 5.18(a) apresenta os histograma da série observada com a curva de uma distribuição estável ajustada pelo método de Koutrouvelis (1980). A estimativa para o parâmetro de estabilidade obtida por esse método foi $\hat{\alpha}=1.2452$. Observe que tal distribuição de fato parece se ajustar bem aos dados como apontado em Burnecki et al. (2008), onde o valor de $\alpha$ obtido para os dados por eles estudados foi igual a 1.2674. O gráfico dos quantís da distribuição estável ajustada (obtidos numericamente) contra os quantís empíricos da série observada é apresentado na Figura 5.18(b) e reforça a suposição de que a distribuição marginal dos dados é estável. Uma análise heurística prévia através da análise R/S é dada na Figura 5.18(c) e fornece um valor alto para o parâmetro de autossimilaridade $H$ porém, o valor estimado de $d$ está próximo de zero, indicando que a série possui memória curta (neste caso, $\hat{d}=\hat{H}-1 / \hat{\alpha}=-0.05<0$ ). Como o método $R / S$ é pouco preciso e seu valor está muito próximo de zero, a análise fornecida por ele neste caso é inconclusiva.

\footnotetext{
${ }^{2}$ http://www.ngdc.noaa.gov/nndc/struts/results?t $=102827 \& \mathrm{~s}=1 \& \mathrm{~d}=8,6,9$
} 


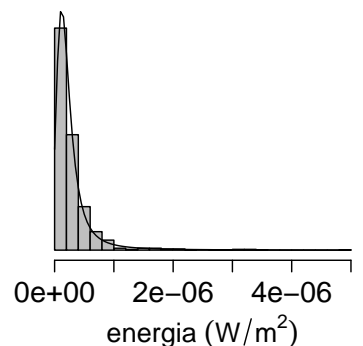

(a)

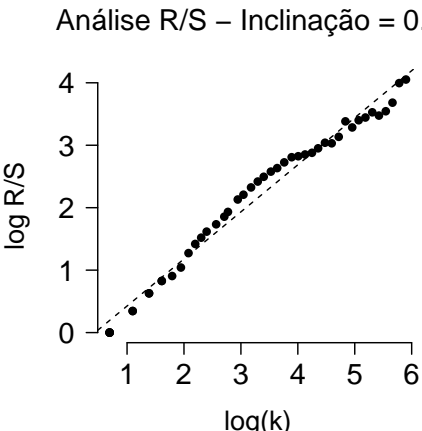

$\log (k)$

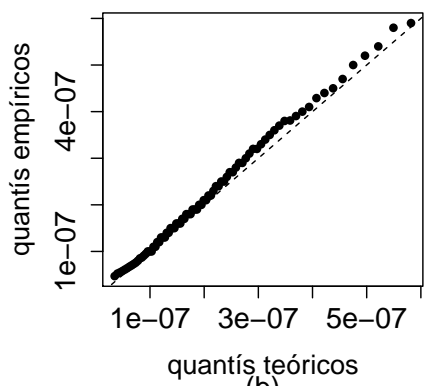

(b)

Posteriori ABC para $\alpha$

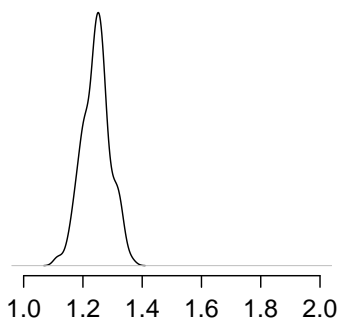

$\left(\begin{array}{l}\alpha \\ (d)\end{array}\right.$

Figura 5.18: (a) Histograma da série observada de erupções solares e curva da densidade estável ajustada. (b) Gráfico dos quantís teóricos da distribuição estável ajustada aos dados contra os quantís empíricos da série observada. (c) Análise $R / S$ aplicada à série observada. (d) Posteriori aproximada para $\alpha$ obtida pelo método $A B C$ semiautomático.

Aplicamos o método ABC 2 utilizado no estudo de simulação da seção anterior obtendo as posterioris aproximadas para $\alpha$ e $H$. No caso de $\alpha$, sua média aproximada a posteriori é igual a 1.2444, um valor próximo do obtido pelo método de Koutrouvelis (1980). A densidade de sua posteriori aproximada é dada na Figura 5.18(d).

As estimativas obtidas para $H$ segundo cada método são dadas na Tabela 5.3. Note que com exceção do método da Variação- $p$, todos os estimadores clássicos retornaram estimativas para $H$ maiores que 1 (lembre que $H<1$ por definição). No caso dos estimadores de ondaleta e FELW, vimos no estudo de simulação que esses possuem alta variabilidade, o que pode explicar o valor maior que 1 estimado se o verdadeiro valor de $H$ estiver próximo de 1 . No caso dos estimadores MSD e var- $p$, vimos que estes deveriam subestimar $H$ quando $\alpha$ e $H$ se aproximam de 1 , mas isso aconteceu apenas com a estimativa obtida pelo método var- $p$. A estimativa para $H$ obtida nos dados de Burnecki et al. (2008) foi 0.9990. Em todos os casos temos $\hat{d}>0$, indicando que os dados exibem memória longa, contrariando a análise $\mathrm{R} / \mathrm{S}$ feita anteriormente.

As estimativas obtidas pelo método ABC estão de acordo com as estimativas obtidas por seus competidores. Como a priori para $H$ é limitada a valores menores que 1 , os métodos $\mathrm{ABC}$ não fornecem estimativas maiores que 1. A Figura 5.19 apresenta a densidade a posteriori aproximada obtida por ABC.

\begin{tabular}{lc}
\hline \hline Método de estimação & $\hat{H}$ \\
\hline Var- $p$ & 0.9400 \\
MSD & 1.2428 \\
FELW & 1.2832 \\
Wave & 1.0352 \\
SAABC 2 & 0.9735 \\
\hline \hline
\end{tabular}

Tabela 5.3: Estimativas de $H$ obtidas pelos métodos de estimação apresentados na seção 5.5. 
Posteriori para H - ABC 2

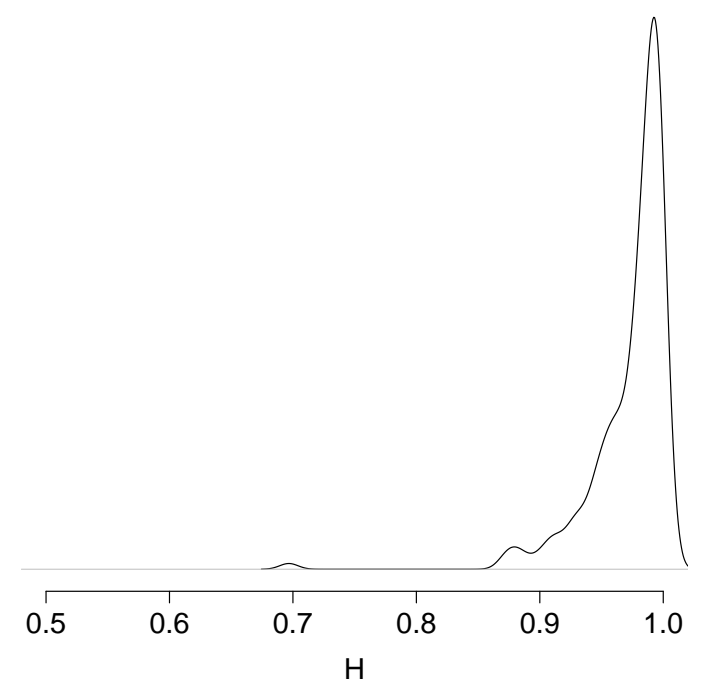

Figura 5.19: Posteriori aproximada para $H$ obtida pelo método ABC 2 apresentado na seção 5.5.

\subsection{Processo gama modulado generalizado}

Nesta seção estudaremos a perfomance do método ABC aplicado aos incrementos do processo gama modulado generalizado (GGMn) apresentado na seção 2.2.4. Até onde sabemos, não há na literatura métodos clássicos disponíveis para comparar nossos resultados, mas apresentaremos aqui uma abordagem alternativa à apresentada em Andrade et al. (2015).

Considere o processo $\left\{Y_{t}, t>0\right\}$ tal que $Y_{t}=\mu+\sigma \Delta\left({ }^{\beta} X_{t}\right)$ onde $\Delta\left({ }^{\beta} X_{t}\right)={ }^{\beta} X_{t}-{ }^{\beta} X_{t-1}$ e ${ }^{\beta} X_{t}$ é um GGMp. Neste caso $\theta=(\beta, \mu, \sigma)$.

Para os parâmetros de localização e escala, é possível obter uma família de distribuições conjugadas, como demonstrado em Iglesias et al. (2011) e Andrade et al. (2015). Neste caso, considerando $\beta$ fixo, as posterioris para $\mu$ e $\sigma$ são obtidas através de um amostrador de Gibbs. O mesmo não ocorre para o parâmetro $\beta$, pois sua verossimilhança não é tratável analiticamente, o que motiva o uso de métodos ABC. Neste caso, precisamos obter alguma informação sobre $\beta$ através de alguma(s) estatística(s). Como $\beta$ é um parâmetro de memória, as estatísticas utilizadas nas seções anteriores são escolhas naturais. No entanto, devido à não estacionariedade e heterocedasticidade inerentes ao processo, o uso dessas estatísticas em conjunto com o $\mathrm{ABC}$ não produzem resultados satisfatórios.

Como mencionado na seção 2.2.4, assintoticamente, a variância do GGMn é uma função potência de $\beta$, implicando que o gráfico da variância do processo contra o tempo, em escala logarítmica, é uma reta com inclinação proporcional a $\beta$. A Figura 2.10 apresentada na mesma seção demonstra esse resultado empíricamente, de onde concluímos que para $t>20, \operatorname{Var}\left[\Delta\left({ }^{\beta} X_{t}\right)\right] \sim t^{2 \beta}$. A exploração deste resultado viabiliza o uso do método $\mathrm{ABC}$ para estimar $\beta$ com uma precisão notável. Além disso, podemos estimar este parâmetro através do método $\mathrm{ABC}$ independentemente dos valores de $\mu$ e $\sigma$ pois, neste caso, para $t$ grande, teremos $\operatorname{Var}\left(Y_{t}\right) \sim \sigma^{2} t^{2 \beta}$ e assim, $\log \operatorname{Var}\left(Y_{t}\right) \sim 2 \log \sigma+2 \beta \log t$, ou seja, a inclinação da reta continua sendo proporcional a $\beta$.

Como observamos apenas uma trajetória do GGMn, temos que a variância de cada ponto amostral é nula. No entanto, dada uma trajetória amostral de tamanho $n$ e um número inteiro $m<<n$, podemos aproximar a variância do processo padrão da seguinte maneira:

- remova os 20 primeiros pontos amostrais (pois devemos ter $\mathrm{t}>20$ ). Seja $N=n-20$;

- divida essa nova trajetória em $[N / m] m$ blocos consecutivos e disjuntos de tamanho $m$ (o símbolo $[\xi]$ denota a parte inteira de $\xi$ ); 
- calcule a variância amostral $s^{2}(k)$ de cada bloco. Esses valores são utilizados como estimativas para as variâncias nos tempos $m, 2 m, \ldots,[N / m] m$;

- para $k=1, \ldots,[N / m]$, a reta de regressão ajustada aos pontos $\left(k m, s^{2}(k)\right)$, em escala logarítmica, deve ter uma inclinação que aproxima o valor $2 \beta$.

O tamanho $m$ dos blocos pode ser escolhido variando $m$ desde que se tenha $m<<n$, por exemplo $m \in\{10,20, \ldots, 100\}$ e $n=1000$. A escolha pode ser feita seguindo algum critério de informação como AIC ou BIC em cada ajuste para cada valor de $m$. Isso pode ser feito iterativamente para cada amostra simulada, mas notamos que o valor $m=20$ produz bons resultados para amostras de tamanho $n \approx 1000$.

A Figura 5.20 apresenta os pontos $\left(k m, s^{2}(k)\right)$ em escala logarítmica com $n=1000(N=980)$ e $m=20$ para trajetórias simuladas do GGMn com diferentes valores de $\beta$ e suas respectivas retas estimadas por mínimos quadrados (linha cheia). A linha tracejada corresponde à linha teórica.
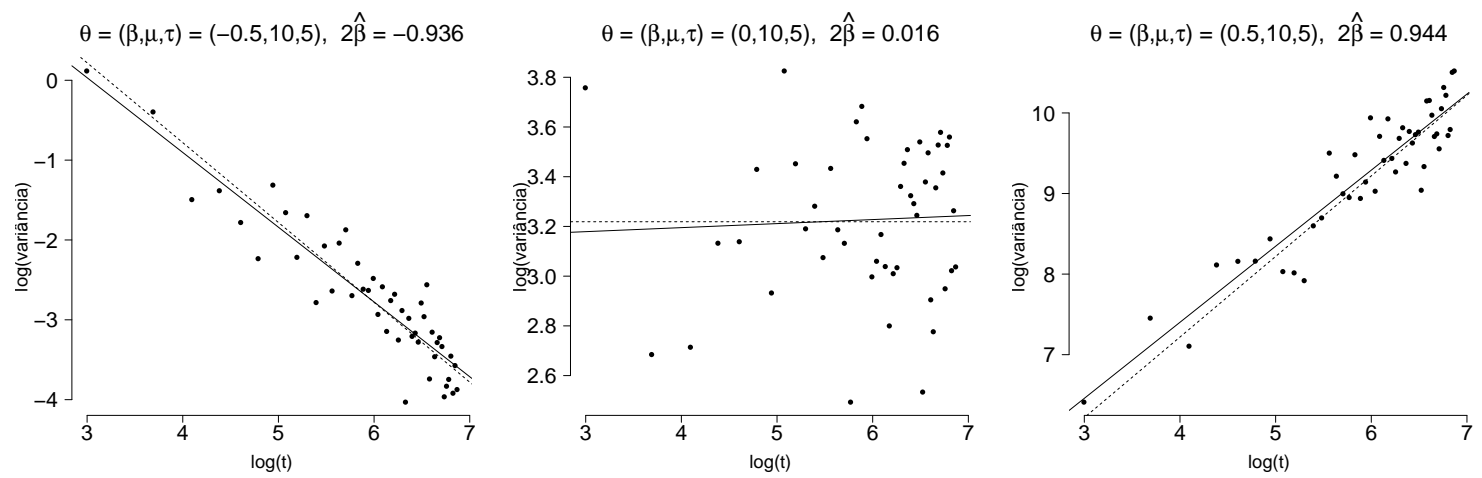

Figura 5.20: Variância em blocos para séries simuladas do GGMn para diferentes valores de $\beta$ em escala logaritmica. A linha cheia corresponde à reta ajustada aos pontos por mínimos quadrados, enquanto a linha tracejada corresponde à linha teórica cuja inclinação é $2 \beta$.

Simulamos 10000 valores de $\theta=(\beta, \mu, \sigma)$ uniformemente no cubo $[-1,1] \times[-100,100] \times[0,100]$ e simulamos uma trajetória do GGMn para cada valor de $\theta$ escolhido. A Figura 5.21(a) apresenta os valores de $\beta$ simulados plotados contra as respectivas aproximações para $\beta$ obtidas pelo método acima. Podemos ver que a inclinação da reta obtida por esse método é de fato informativa com respeito à $\beta$ sendo levemente imprecisa nos pontos extremos -1 e 1 . O mesmo foi feito para cada $\mu$ (Figura 5.21(b)) escolhido plotado contra a média amostral de cada trajetória e, como esperado, a média amostral é altamente informativa para $\mu$.

Embora fosse esperado que o intercepto estimado pelo método acima fosse informativo para o parâmetro de escala $\sigma$, já que seu valor teórico é igual a $2 \log (\sigma)$, a Figura 5.21(c) mostra que esses valores não conseguem discriminar valores diferentes de $\sigma$ efetivamente. Neste gráfico foram plotados os valores de $\sigma$ simulados contra a exponencial dos interceptos estimados dividos por 2 .

Optamos por estimar os parâmetros do modelo como em Andrade et al. (2015), isto é, estimando $\beta$ através do algoritmo $\mathrm{D}$, já que este método de estimação independe dos valores de $\mu \mathrm{e}$ $\sigma$ da trajetória observada, para em seguida estimar $\mu$ e $\sigma$ utilizando a posteriori obtida para $\beta$. Como mencionado anteriormente, Andrade et al. (2015) utilizaram um amostrador de Gibbs para estimar os parâmetros de localização e escala obtendo resultados razoáveis. No entanto, a abordagem lá apresentada aplica-se apenas para $\beta \in[-1,0]$, pois para $\beta \in(0,1]$ a matriz de covariância utilizada na distribuição que modela os dados torna-se computacionalmente singular, o que impede o cálculo numérico de sua inversa.

Ao invés de utilizar um amostrador de Gibbs para estimar $\mu$ e $\sigma$, vamos utilizar o método ABC semiautomático. Os resultados obtidos para $\beta \leq 0$ serão comparados com os obtidos em Andrade et al. (2015), mas apresentaremos também os resultados para $\beta>0$.

Para $\beta$ utilizamos o mesmo método de Andrade et al. (2015), isto é, implementamos o algoritmo $\mathrm{D}$ tendo como estatística resumo a inclinação da reta obtida pelo método descrito anteriormente. 


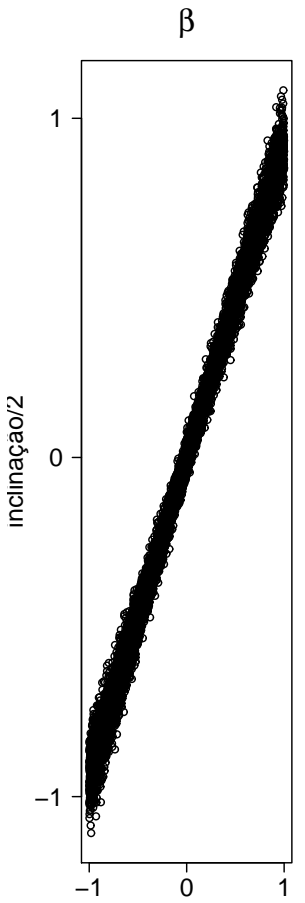

(a)

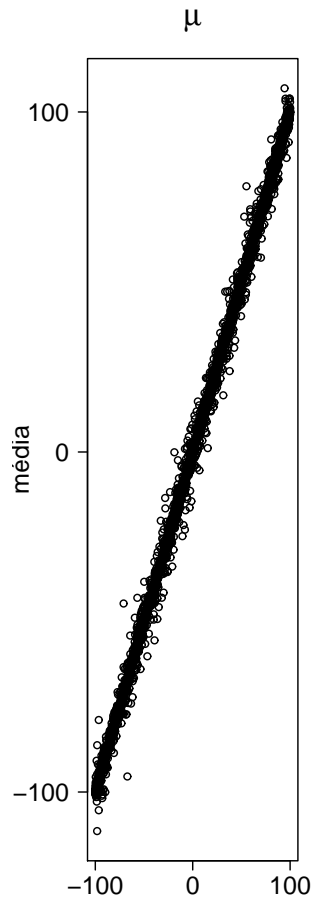

(b)

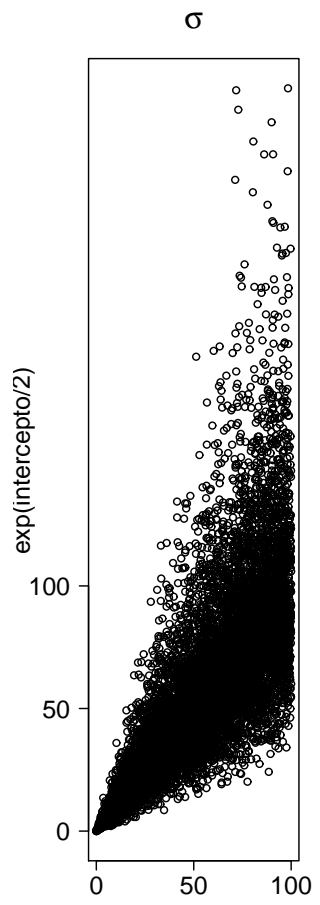

(c)

Figura 5.21: Gráficos dos parâmetros $(\beta, \mu, \sigma)$ simulados da priori plotados contra os valores de suas respectivas estatísticas resumo calculadas em 10000 simulações do processo GGMn.

Neste caso, a priori para $\beta$ é uniforme no intervalo $[-1,1]$. A Figura 5.22 nos mostra os boxplots da distribuição amostral da média a posteriori para $\beta$ obtidas através de simulações de Monte Carlo com $\beta$ variando em $\{-1,-0.8, \ldots, 0.8,1\}$. Note que as estimativas obtidas pelo algoritmo $\mathrm{D}$ são razoáveis para todos os valores do parâmetro em questão, com uma pequena superestimação para $\beta=-1$ e uma pequena subestimação para $\beta=1$.

Calculamos os $e$-valores aproximados (veja a seção 4.3) com respeito à hipótese $\beta_{0}=-0.5$ (processo $t$-Student) com base na amostra a posteriori obtida pelo algoritmo $\mathrm{D}$, quando o valor nominal de $\beta$ varia em $[-1,1]$. A Figura 5.23(a) apresenta a distribuição amostral desses $e$-valores para 200 simulações com $\beta \in\{-1,-0.9, \ldots, 0.9,1\}$. Observe que os $e$-valores obtidos fornecem alta evidência para $\beta_{0}=-0.5$ quando o valor nominal de $\beta$ está próximo de $\beta_{0}$ e baixa evidência caso contrário. A evidência para valores próximos aos extremos -1 e 1 refletem o desempenho do método $\mathrm{ABC}$ para esses valores, mas não prejudicam de forma significativa a análise.

No caso $\beta_{0}=0$ (caso normal), apresentado na Figura 5.23((b), os $e$-valores são menores para $\beta=0$ quando comparado com o caso anterior, mas este ainda fornece uma boa discriminação em favor da hipótese nula.

Para os parâmetros de localização e escala $\mu$ e $\sigma$, aplicamos o método ABC-MCMC apresentado em Andrade et al. (2015) e o método ABC semiautomático (SAABC). Para o método semiautomático, utilizamos as estatísticas de ordem como estatísticas resumo iniciais. A Figura 5.24 apresenta 10000 valores de $(\mu, \sigma)$ gerados uniformemente no plano $[-100,100] \times[0,100]$ plotados contra as estatísticas resumo obtidas pelo método SAABC. Nota-se que estas discriminam bem os valores de $\mu$ e $\sigma$, mas quando $\sigma$ cresce, o método torna-se menos preciso.

A Tabela 5.4 apresenta os resultados obtidos em 200 simulações de Monte Carlo para $\beta$ variando em $\{-1,-0.8, \ldots,-0.2,0\}$ (mesmo resultado para os métodos ABC-MCMC e SAABC) e para $\mu$ e $\sigma$ fixos e iguais a 0 e 1 respectivamente segundo cada método. Neste caso utilizamos priori uniforme no cubo $[-1,0] \times[-100,100] \times[0,100]$ para $(\beta, \mu, \tau)$. Nota-se que as estimativas para $\mu$ são praticamente as mesmas para os dois métodos. No entanto, as estimativas para $\sigma$ são bem melhores quando $\beta$ tende a -1 e o método SAABC é utilizado. Quando $\beta$ tende a 0 (caso normal) os resultados são equivalentes. Este comportamento se repete para outros valores de $\mu$ e $\sigma$. 


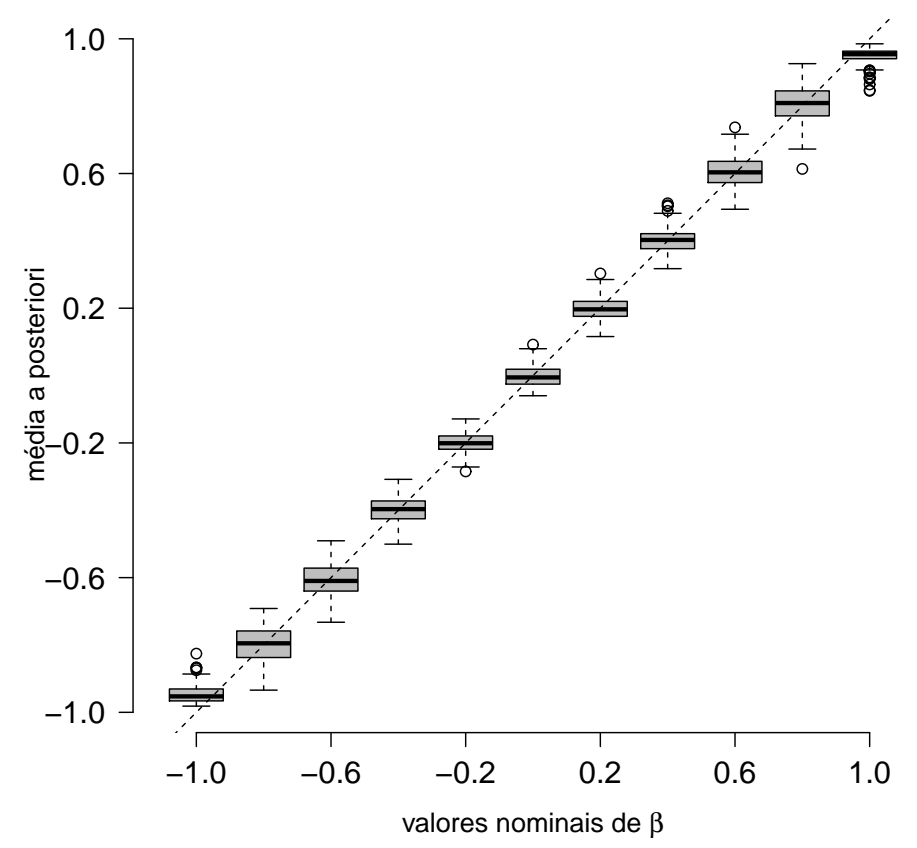

Figura 5.22: Distribuição amostral da média aproximada a posteriori para $\beta$ em 200 simulações de Monte Carlo através do algoritmo $D$ para diferentes valores de $\beta$.

(a)

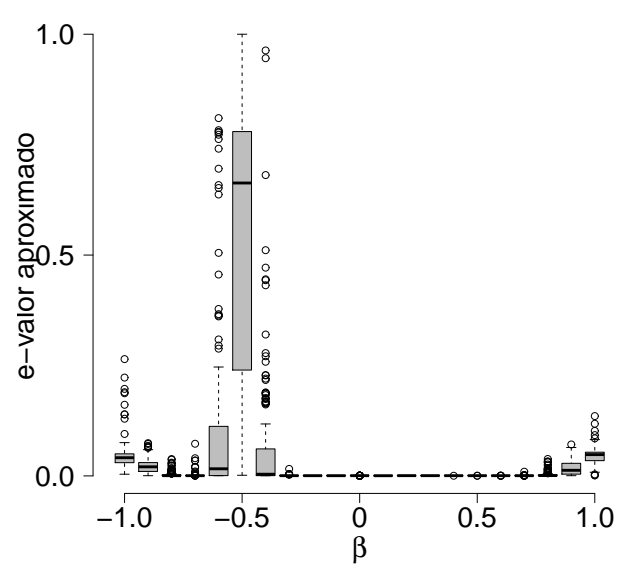

(b)

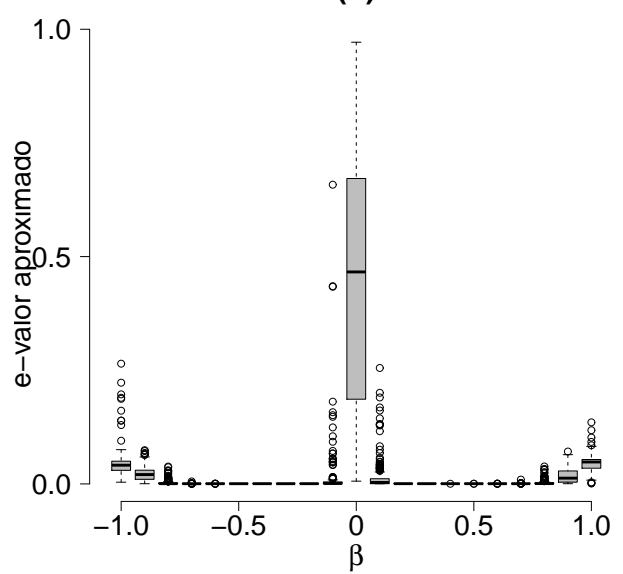

Figura 5.23: Boxplots dos e-valores obtidos a partir da amostra aproximada a posteriori em 200 simulações de Monte Carlo para diferentes valores de $\beta$ : (a) $\beta_{0}=-0.5$; (b) $\beta_{0}=0$.

A Figura 5.25 apresenta os boxplots das estimativas (média a posteriori) para $\mu$ e $\sigma$ obtidas pelo SAABC em 200 simulações de Monte Carlo. Os valores nominais para $\mu$ e $\sigma$ são iguais a 0 e 1 respectivamente e o valor nominal de $\beta$ varia no conjunto $\{-1,-0.8, \ldots, 0.8,1\}$. Os resultados sugerem dois comportamentos distintos quando $\beta$ varia em seu espaço paramétrico: (1) Quando $\beta \rightarrow 1^{-}$, os dados se tornam menos informativos para o parâmetro de localização. Este resultado era esperado devido o comportamento das trajetórias do GGMn, pois quando $\beta \rightarrow-1^{+}$, os incrementos estabilizam rapidamente em torno de $\mu$ e quando $\beta \rightarrow 1^{-}$, o processo de incrementos diverge. (2) Quando $|\beta| \rightarrow 1$ os dados se tornam menos informativo para o parâmetro de escala, embora os resultados ainda sejam razoáveis.

A Tabela A. 6 apresenta os resultados obtidos pelo SAABC para $\mu=10$ e $\sigma=5$ e $\operatorname{com} \beta$ variando em $[-1,1]$, equanto a Figura 5.26 apresenta a distribuição amostral conjunta das estimativas de 
$\mu$

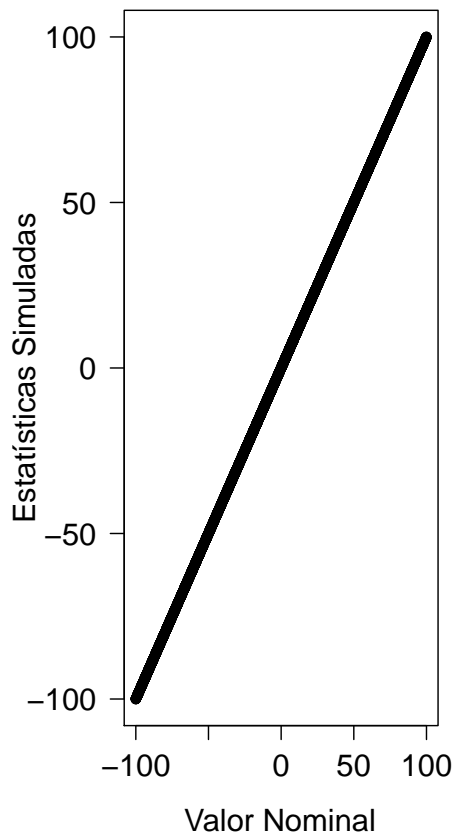

$\sigma$

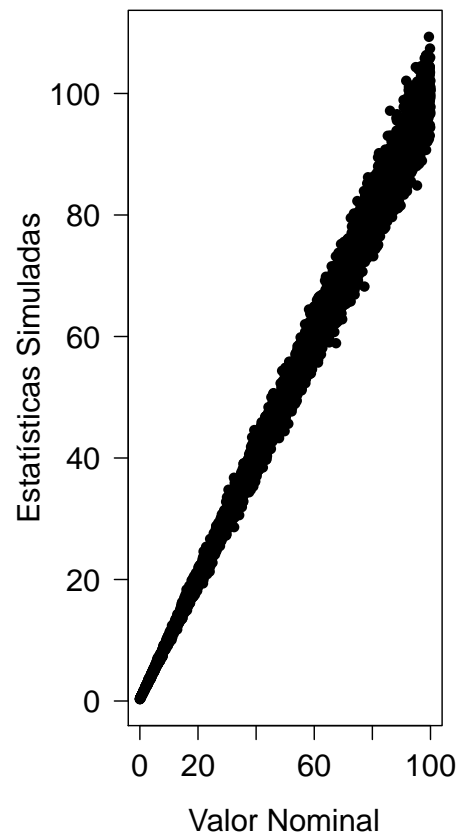

Figura 5.24: Gráficos dos parâmetros $(\mu, \sigma)$ simulados da priori plotados contra os valores de suas respectivas estatísticas resumo obtidas pelo método SAABC.

média a posteriori para $\mu$

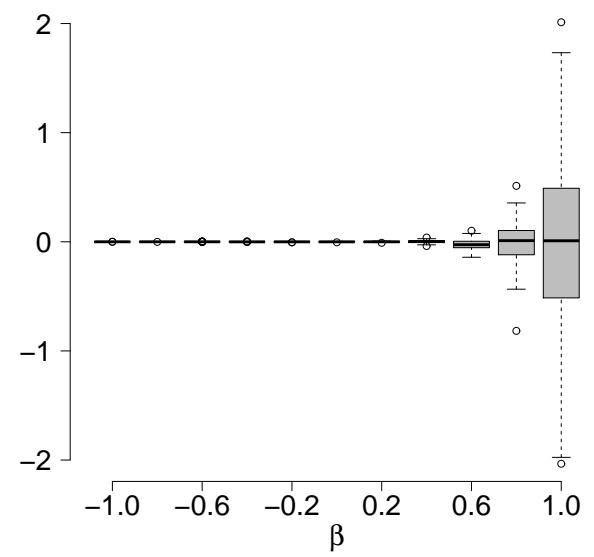

média a posteriori para $\sigma$

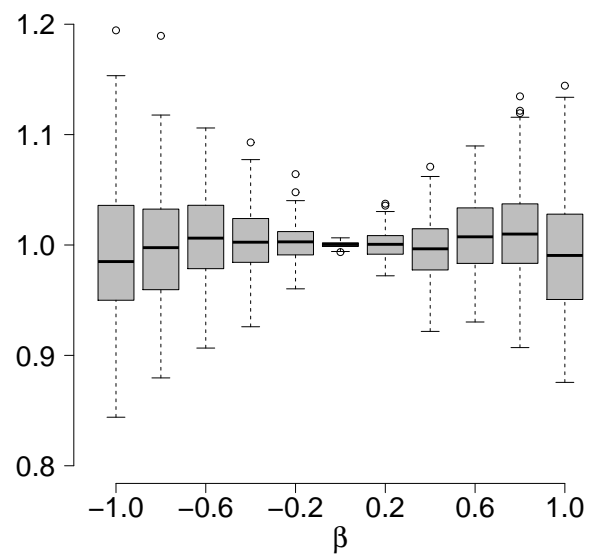

Figura 5.25: Distribuição amostral da média aproximada a posteriori para $\mu$ (esquerda) e $\sigma$ (direita), obtidas em 200 simulações de Monte Carlo variando $\beta$.

$(\mu, \sigma)$ para $\beta \in\{-0.5,-0.1,0.1,0.5\}$. Podemos notar que os resultados são razoáveis em todos os casos.

\subsubsection{Aplicação: Retornos do índice S\&P500}

Este conjunto de dados foi estudado em Iglesias et al. (2011) e consiste de aproximadamente 1000 log-retornos do valor de fechamento (end-of-day) do índice S\&P500 no período de novembro de 2005 a março de 2009. Este conjunto de dados pode ser obtido em https://finance.yahoo.com/ $\mathrm{q} / \mathrm{hp} ? \mathrm{~s}=\% 5 \mathrm{EGSPC}+$ Historical + Prices .

Podemos notar pela série observada (Figura 5.27(a)) que esta possui um comportamento similar ao GGMn com $\beta<0$, ou seja, a variabilidade não é constante e decresce com o tempo. Isso também 


\begin{tabular}{|l|c|cc|cc|}
\hline \hline Método & & ABC-MCMC & SAABC & \\
\hline$\theta$ & $\beta=-\mathbf{1 . 0}$ & $\mu=\mathbf{0}$ & $\sigma=\mathbf{1}$ & $\mu=0$ & $\sigma=\mathbf{1}$ \\
\hline$\hat{\theta}$ & -0.9447 & $-2 \mathrm{e}-06$ & 0.9670 & $3 \mathrm{e}-06$ & 0.9923 \\
$d p$ & 0.0267 & $8 \mathrm{e}-05$ & 0.2403 & $7 \mathrm{e}-05$ & 0.0618 \\
$\sqrt{\mathrm{EQM}}$ & 0.0614 & $8 \mathrm{e}-05$ & 0.2419 & $7 \mathrm{e}-05$ & 0.0622 \\
\hline$\theta$ & $\beta=-\mathbf{0 . 8}$ & $\mu=\mathbf{0}$ & $\sigma=\mathbf{1}$ & $\mu=0$ & $\sigma=\mathbf{1}$ \\
\hline$\hat{\theta}$ & -0.7996 & $-1 \mathrm{e}-05$ & 0.9879 & $1 \mathrm{e}-05$ & 0.9977 \\
$d p$ & 0.0521 & 0.0002 & 0.1857 & 0.0002 & 0.0501 \\
$\sqrt{\mathrm{EQM}}$ & 0.0519 & 0.0002 & 0.1856 & 0.0002 & 0.0500 \\
\hline$\theta$ & $\beta=-\mathbf{0 . 6}$ & $\mu=\mathbf{0}$ & $\sigma=\mathbf{1}$ & $\mu=0$ & $\sigma=\mathbf{1}$ \\
\hline$\hat{\theta}$ & -0.6082 & $-4 \mathrm{e}-05$ & 1.0015 & $-2 \mathrm{e}-05$ & 1.0067 \\
$d p$ & 0.0463 & 0.0005 & 0.1324 & 0.0006 & 0.0404 \\
$\sqrt{\mathrm{EQM}}$ & 0.0469 & 0.0005 & 0.1320 & 0.0006 & 0.0409 \\
\hline$\theta$ & $\beta=-\mathbf{0 . 4}$ & $\mu=\mathbf{0}$ & $\sigma=\mathbf{1}$ & $\mu=0$ & $\sigma=\mathbf{1}$ \\
\hline$\hat{\theta}$ & -0.3994 & $-7 \mathrm{e}-05$ & 1.0082 & -0.0003 & 1.0034 \\
$d p$ & 0.0356 & 0.0011 & 0.0781 & 0.0011 & 0.0286 \\
$\sqrt{\mathrm{EQM}}$ & 0.0355 & 0.0011 & 0.0783 & 0.0011 & 0.0287 \\
\hline$\theta$ & $\beta=-\mathbf{0 . 2}$ & $\mu=\mathbf{0}$ & $\sigma=\mathbf{1}$ & $\mu=0$ & $\sigma=\mathbf{1}$ \\
\hline$\hat{\theta}$ & -0.1995 & $-5 \mathrm{e}-05$ & 1.0065 & -0.0003 & 1.0023 \\
$d p$ & 0.0286 & 0.0021 & 0.0380 & 0.0015 & 0.0168 \\
$\sqrt{\mathrm{EQM}}$ & 0.0285 & 0.0021 & 0.0385 & 0.0015 & 0.0168 \\
\hline$\theta$ & $\beta=\mathbf{0 . 0}$ & $\mu=\mathbf{0}$ & $\sigma=\mathbf{1}$ & $\mu=0$ & $\sigma=\mathbf{1}$ \\
\hline$\hat{\theta}$ & -0.0011 & 0.0001 & 0.9992 & -0.0001 & 1.0002 \\
$d p$ & 0.0313 & 0.0015 & 0.0019 & 0.0015 & 0.0024 \\
$\sqrt{\mathrm{EQM}}$ & 0.0313 & 0.0015 & 0.0020 & 0.0015 & 0.0024 \\
\hline \hline
\end{tabular}

Tabela 5.4: Resultados obtidos em 200 simulações de Monte Carlo utilizando-se os métodos ABC-MCMC de Andrade et al. (2015) e SAABC.

é observado no gráfico das variâncias em blocos dado pela Figura 5.27(b), ou seja, os dados sugerem que o processo que os gerou possui variância proporcional a $t^{x}$, com $x<0$. Iglesias et al. (2011) consideram como modelo o processo $t$-Student, isto é, um GGMn com $\beta$ fixo e igual a -0.5 . Vamos verificar se tal suposição é razoável, aplicando o método SAABC.

Utilizamos o algoritmo D para estimar $\beta$ obtendo a sua posteriori marginal para, em seguida, obter as posterioris de $\mu$ e $\sigma$ pelo SAABC. A Figura 5.28 apresenta essas posterioris. As estimativas pontuais obtidas (média a posteriori) foram $\hat{\beta}=-0.5799( \pm 0.0463), \hat{\mu}=-0.0002( \pm 0.0003)$ e $\hat{\sigma}=$ $0.2400( \pm 0.0197)$. Os intervalos de credibilidade de $95 \%$ para $\beta, \mu$ e $\sigma$ são dados, respectivamente, por $(-0.6701 ;-0.4922),(-0.0010 ; 0.0004)$ e $(0.1976 ; 0.2860)$. Observe que o intervalo de credibilidade para $\beta$ contém o valor -0.5 utilizado em Iglesias et al. (2011), mas o $e$-valor aproximado calculado é igual a 0.1098 , ou seja, uma evidência em favor da hipótese $\beta_{0}=-0.5$ que pode ser considerada baixa. $\mathrm{O}$ valor de $\sigma$ estimado parece cobrir de maneira efetiva toda a variabilidade presente nos dados.

\subsection{Considerações Finais}

Neste trabalho propomos o uso de uma classe de métodos Bayesianos conhecida como ABC para estudar a memória de alguns processos com memória longa em situações onde a função de verossimilhança é intratável analiticamente ou inexistente. A abordagem proposta mostrou-se eficaz quando comparada com os principais métodos de estimação clássicos encontrados na literatura. Essa eficácia foi comprovada através da aplicação do método em dados sintéticos e reais. Embora a abordagem apresentada tenha sido aplicada apenas em algumas classes de processos com memória 

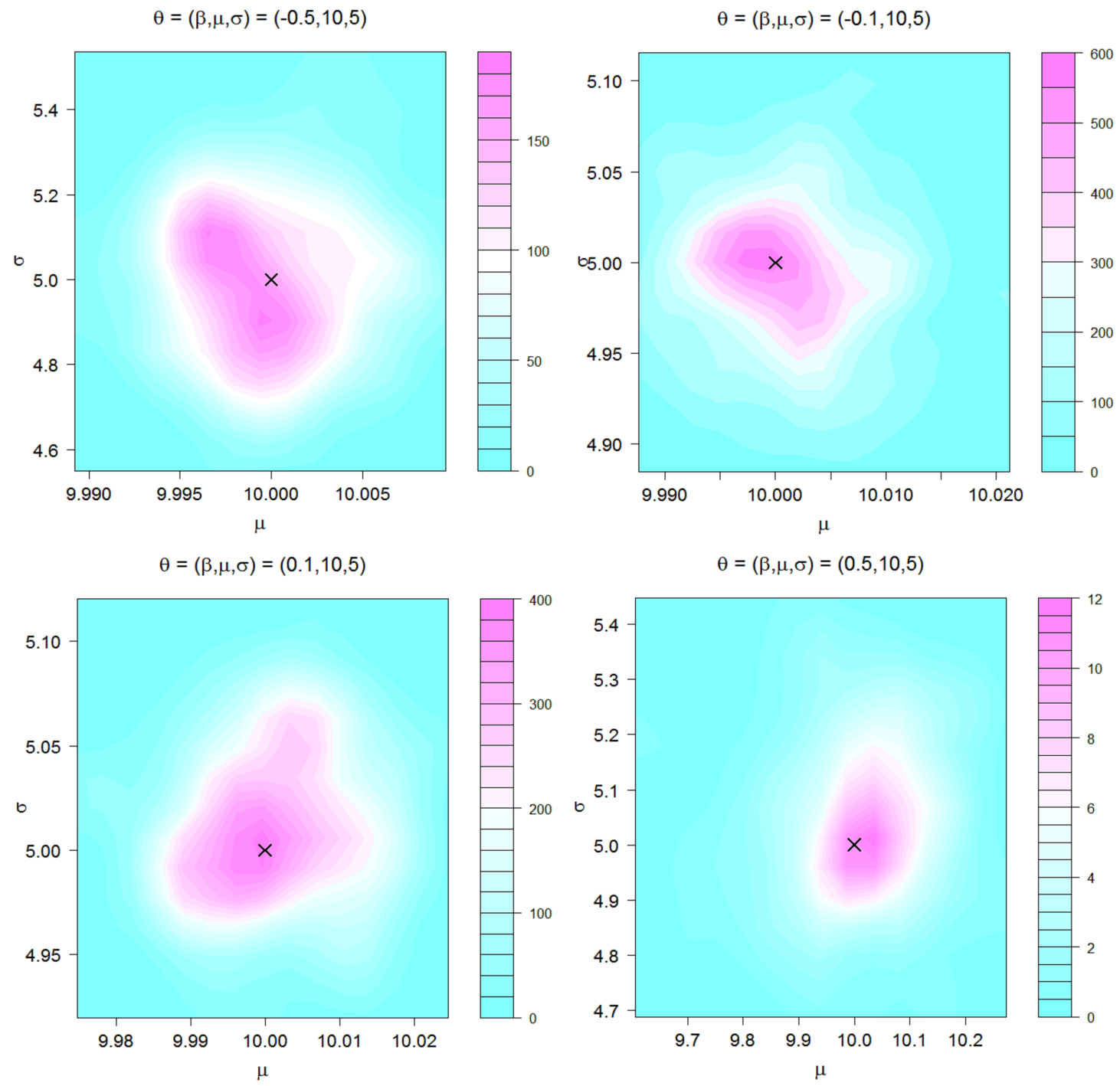

Figura 5.26: Niveis das distribuições amostrais conjuntas de $(\mu, \sigma)$ obtidas pelo método $S A A B C$ em 200 simulações de Monte Carlo para diferentes valores de $\beta$.

(a) log-retornos do índice S\&P500

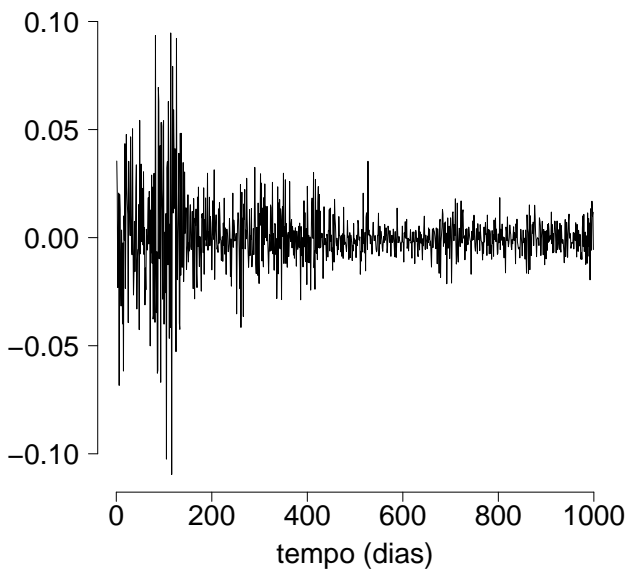

(b) inclinação $=-1.0680$

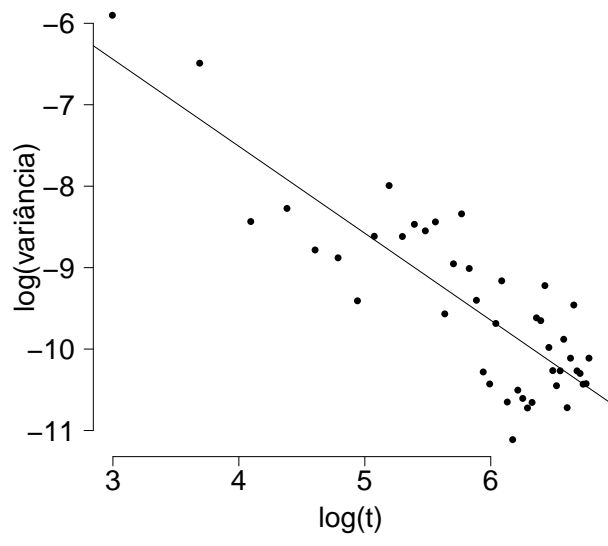

Figura 5.27: (a) log-retornos dos valores de fechamento do indice SEP500 no período de novembro de 2005 a março de 2009. (b) Variância em blocos da série observada e reta ajustada por mínimos quadrados. 


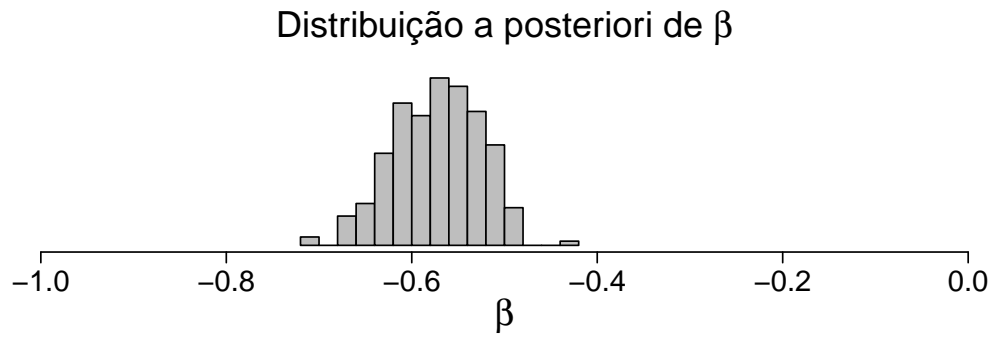

Distribuição a posteriori de $\mu$

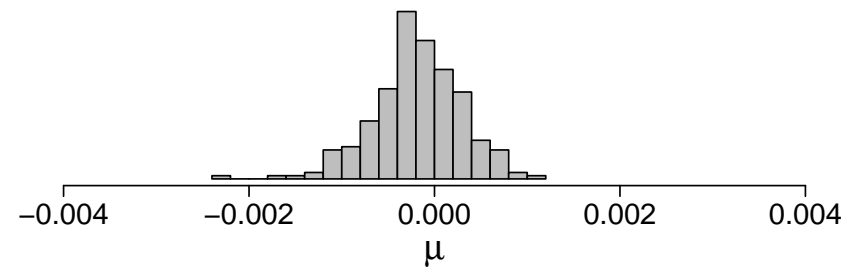

Distribuição a posteriori de $\sigma$

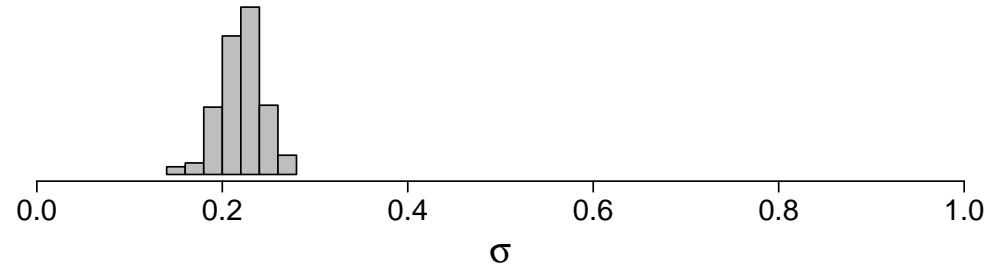

Figura 5.28: Distribuiçôes a posteriori aproximadas obtidas para os parâmetros $(\beta, \mu, \sigma)$ para os dados do indice S\&P500.

longa, esta pode ser aplicada em qualquer outro processo com memória longa, desde que seja possível simular deste processo e que as devidas modificações sejam feitas. Essas modificações basicamente consistem em obter boas estatísticas resumo para os parâmetros de interesse.

Com base nos resultados obtidos, acreditamos que seja possível realizar boas inferências para processos complexos utilizando métodos baseados em ABC.

\subsection{Sugestões para Pesquisas Futuras}

Como mencionado na seção anterior, a abordagem proposta pode ser estendida a uma ampla classe de processos com memória longa. Especialmente em processos que apresentem certas propriedades, como autossimilaridade. Deste modo, muito do que foi apresentado aqui pode ser reaproveitado.

Existe na literatura uma grande quantidade de processos com memória longa que pouco foram estudados do ponto de vista inferencial com respeito ao parâmetro de memória. Alguns exemplos incluem:

- Processo de Poisson fracionário (Cahoy et al., 2010). Tal processo generaliza o processo de Poisson usual para o caso com memória longa;

- Modificações do GGMp (veja o apêndice de Andrade et al. (2015));

- Modelos FARIMA (Beran, 1994; Beran et al., 2013) com inovações estáveis ou dadas pela distribuição de Rosenblatt. Neste caso a memória de curto alcance também é modelada.

Além disso, podemos avaliar neste contexto a eficácia dos métodos ABC na escolha de modelos e testes de hipóteses Bayesianos. 
APLICAÇÕES E CONCLUSÕES 


\section{Apêndice A}

\section{Tabelas de Resultados}

\begin{tabular}{llcccc}
\hline \hline Método de Estimação & & $\mathbf{0 . 2}$ & $\mathbf{0 . 5}$ & $\mathbf{0 . 7}$ & $\mathbf{0 . 9}$ \\
\hline Variograma & $\hat{\mathbf{H}}$ & 0.1710 & 0.4499 & 0.6139 & 0.7466 \\
& $d p$ & 0.0758 & 0.0981 & 0.1039 & 0.1034 \\
& $\sqrt{\mathrm{EQM}}$ & 0.0810 & 0.1100 & 0.1345 & 0.1849 \\
\hline $\mathbf{R} / \mathbf{S}$ & $\hat{\mathbf{H}}$ & 0.3369 & 0.5601 & 0.7034 & 0.8275 \\
& $d p$ & 0.0585 & 0.0799 & 0.0884 & 0.0904 \\
& $\sqrt{\mathrm{EQM}}$ & 0.1488 & 0.0995 & 0.0883 & 0.1157 \\
\hline Periodograma & $\hat{\mathbf{H}}$ & 0.1602 & 0.5056 & 0.7263 & 0.9476 \\
& $d p$ & 0.0777 & 0.0792 & 0.0783 & 0.0785 \\
& $\sqrt{\mathrm{EQM}}$ & 0.0871 & 0.0794 & 0.0825 & 0.0916 \\
\hline DFA & $\hat{\mathbf{H}}$ & 0.2166 & 0.5000 & 0.6935 & 0.8765 \\
& $d p$ & 0.0230 & 0.0407 & 0.0526 & 0.0598 \\
Whittle & $\sqrt{\mathrm{EQM}}$ & 0.0283 & 0.0400 & 0.0529 & 0.0640 \\
\hline FEXP & $\hat{\mathbf{H}}$ & 0.1938 & 0.4980 & 0.6975 & 0.8950 \\
& $d p$ & 0.0162 & 0.0195 & 0.0206 & 0.0206 \\
& $\sqrt{\mathrm{EQM}}$ & 0.0173 & 0.0200 & 0.0200 & 0.0200 \\
\hline FELW & $\hat{\mathbf{H}}$ & 0.2070 & 0.4999 & 0.6963 & 0.9001 \\
& $d p$ & 0.0921 & 0.0912 & 0.0906 & 0.0931 \\
& $\sqrt{\mathrm{EQM}}$ & 0.0921 & 0.0911 & 0.0905 & 0.0933 \\
\hline Ondaleta & $\hat{\mathbf{H}}$ & 0.1745 & 0.4962 & 0.6965 & 0.9049 \\
& $d p$ & 0.0617 & 0.0588 & 0.0606 & 0.0628 \\
& $\sqrt{\mathrm{EQM}}$ & 0.0666 & 0.0592 & 0.0607 & 0.0632 \\
\hline ABC & $\hat{\mathbf{H}}$ & 0.1133 & 0.4811 & 0.6914 & 0.9055 \\
& $d p$ & 0.0690 & 0.0600 & 0.0522 & 0.0510 \\
& $\sqrt{\mathrm{EQM}}$ & 0.1107 & 0.0632 & 0.0529 & 0.0509 \\
\hline \hline & $\hat{\mathbf{H}}$ & 0.1999 & 0.5003 & 0.6998 & 0.9002 \\
& $d p$ & 0.0044 & 0.0035 & 0.0030 & 0.0022 \\
& $\sqrt{\mathrm{EQM}}$ & 0.0043 & 0.0035 & 0.0030 & 0.0022 \\
\hline & $\hat{\mathbf{H}}$ & 0.1908 & 0.5006 & 0.7003 & 0.9003 \\
& $d p$ & 0.0183 & 0.0072 & 0.0057 & 0.0038 \\
& $\sqrt{\mathrm{EQM}}$ & 0.0204 & 0.0072 & 0.0056 & 0.0037 \\
\hline & & & & & \\
& & & & & \\
& & & &
\end{tabular}

Tabela A.1: Resultados obtidos para $H$ através de diferentes métodos em 200 simulações de Monte Carlo do ruído Gaussiano fracionário ( $f G n$ ). 


\begin{tabular}{llcccc}
\hline \hline Método de Estimação & & $\mathbf{0 . 5 5}$ & $\mathbf{0 . 7 5}$ & $\mathbf{0 . 8 5}$ & $\mathbf{0 . 9 5}$ \\
\hline Variograma & $\hat{\mathbf{H}}$ & 0.4890 & 0.5892 & 0.6582 & 0.7441 \\
& $d p$ & 0.1102 & 0.1280 & 0.1451 & 0.1163 \\
& $\sqrt{\mathrm{EQM}}$ & 0.1257 & 0.2057 & 0.2402 & 0.2364 \\
\hline $\mathbf{R} / \mathbf{S}$ & $\hat{\mathbf{H}}$ & 0.6025 & 0.7054 & 0.7517 & 0.8246 \\
& $d p$ & 0.0817 & 0.0919 & 0.0988 & 0.0944 \\
& $\sqrt{\mathrm{EQM}}$ & 0.0969 & 0.1020 & 0.1393 & 0.1568 \\
\hline Periodograma & $\hat{\mathbf{H}}$ & 0.5619 & 0.7296 & 0.8417 & 0.9599 \\
& $d p$ & 0.0904 & 0.1078 & 0.1139 & 0.1101 \\
& $\sqrt{\mathrm{EQM}}$ & 0.0911 & 0.1095 & 0.1140 & 0.1104 \\
\hline DFA & $\hat{\mathbf{H}}$ & 0.5439 & 0.6637 & 0.7350 & 0.8057 \\
& $d p$ & 0.0568 & 0.0770 & 0.0818 & 0.0667 \\
& $\sqrt{\mathrm{EQM}}$ & 0.0566 & 0.1153 & 0.1411 & 0.1587 \\
\hline Whittle & $\hat{\mathbf{H}}$ & 0.5760 & 0.7347 & 0.8275 & 0.9216 \\
& $d p$ & 0.0306 & 0.0527 & 0.0519 & 0.0418 \\
& $\sqrt{\mathrm{EQM}}$ & 0.0400 & 0.0548 & 0.0566 & 0.0500 \\
\hline FEXP & $\hat{\mathbf{H}}$ & 0.5379 & 0.6875 & 0.7970 & 0.9206 \\
& $d p$ & 0.1056 & 0.1205 & 0.1378 & 0.1267 \\
& $\sqrt{\mathrm{EQM}}$ & 0.1058 & 0.1356 & 0.1473 & 0.1296 \\
\hline FELW & $\hat{\mathbf{H}}$ & 0.5477 & 0.7068 & 0.8088 & 0.9230 \\
& $d p$ & 0.0707 & 0.0928 & 0.1008 & 0.0936 \\
& $\sqrt{\mathrm{EQM}}$ & 0.0707 & 0.1019 & 0.1086 & 0.0969 \\
\hline Ondaleta & $\hat{\mathbf{H}}$ & 0.5542 & 0.7209 & 0.8200 & 0.9326 \\
& $d p$ & 0.0648 & 0.0799 & 0.0724 & 0.0830 \\
& $\sqrt{\mathrm{EQM}}$ & 0.0648 & 0.0848 & 0.0781 & 0.0848 \\
\hline ABC & $\hat{\mathbf{H}}$ & 0.5670 & 0.7562 & 0.8507 & 0.9300 \\
& $d p$ & 0.0322 & 0.0581 & 0.0505 & 0.0353 \\
& $\sqrt{\mathrm{EQM}}$ & 0.0364 & 0.0583 & 0.0503 & 0.0405 \\
\hline SAABC & $\hat{\mathbf{H}}$ & 0.5680 & 0.7645 & 0.8651 & 0.9214 \\
& $d p$ & 0.0264 & 0.0413 & 0.0415 & 0.0444 \\
& $\sqrt{\mathrm{EQM}}$ & 0.0319 & 0.0471 & 0.0441 & 0.0527 \\
\hline \hline & & & & & \\
& & & & & \\
& & &
\end{tabular}

Tabela A.2: Resultados obtidos para $H$ através de diferentes métodos em 200 simulações de Monte Carlo dos incrementos do processo de Rosenblatt (Rn). 


\begin{tabular}{llcccc}
\hline \hline Método de Estimação & & $\mathbf{0 . 2}$ & $\mathbf{0 . 5}$ & $\mathbf{0 . 7}$ & $\mathbf{0 . 9}$ \\
\hline Variograma & $\hat{\mathbf{H}}$ & 0.3869 & 0.4484 & 0.5792 & 0.7295 \\
& $d p$ & 0.0952 & 0.0945 & 0.1130 & 0.1086 \\
& $\sqrt{\mathrm{EQM}}$ & 0.2097 & 0.1077 & 0.1652 & 0.2020 \\
\hline $\mathbf{R} / \mathbf{S}$ & $\hat{\mathbf{H}}$ & 0.4812 & 0.5687 & 0.6547 & 0.7935 \\
& $d p$ & 0.0879 & 0.0814 & 0.0940 & 0.1239 \\
& $\sqrt{\mathrm{EQM}}$ & 0.2945 & 0.1063 & 0.1039 & 0.1634 \\
\hline Periodograma & $\hat{\mathbf{H}}$ & 0.4041 & 0.5027 & 0.6782 & 0.8725 \\
& $d p$ & 0.0732 & 0.0790 & 0.0736 & 0.0743 \\
& $\sqrt{\mathrm{EQM}}$ & 0.2168 & 0.0787 & 0.0781 & 0.0794 \\
\hline DFA & $\hat{\mathbf{H}}$ & 0.9605 & 0.9530 & 0.9484 & 0.9515 \\
& $d p$ & 0.0047 & 0.0060 & 0.0081 & 0.0153 \\
& $\sqrt{\mathrm{EQM}}$ & 0.7605 & 0.4531 & 0.2486 & 0.0538 \\
\hline Whittle & $\hat{\mathbf{H}}$ & 0.3487 & 0.4985 & 0.6352 & 0.7714 \\
& $d p$ & 0.0174 & 0.0211 & 0.0210 & 0.0215 \\
& $\sqrt{\mathrm{EQM}}$ & 0.1496 & 0.0200 & 0.0678 & 0.1304 \\
\hline FEXP & $\hat{\mathbf{H}}$ & 0.4356 & 0.5014 & 0.6619 & 0.8670 \\
& $d p$ & 0.0873 & 0.0912 & 0.0882 & 0.0934 \\
& $\sqrt{\mathrm{EQM}}$ & 0.2512 & 0.0911 & 0.0959 & 0.0990 \\
\hline FELW & $\hat{\mathbf{H}}$ & 0.4097 & 0.4957 & 0.6545 & 0.8467 \\
& $d p$ & 0.0551 & 0.0589 & 0.0592 & 0.0575 \\
& $\sqrt{\mathrm{EQM}}$ & 0.2168 & 0.0592 & 0.0748 & 0.0781 \\
\hline Ondaleta & $\hat{\mathbf{H}}$ & 0.3550 & 0.4830 & 0.6359 & 0.8029 \\
& $d p$ & 0.0746 & 0.0676 & 0.0573 & 0.0643 \\
& $\sqrt{\mathrm{EQM}}$ & 0.1719 & 0.0693 & 0.0860 & 0.1166 \\
\hline ABC & $\hat{\mathbf{H}}$ & 0.1997 & 0.4953 & 0.6976 & 0.8998 \\
& $d p$ & 0.0425 & 0.0366 & 0.0400 & 0.0337 \\
& $\sqrt{\mathrm{EQM}}$ & 0.0423 & 0.0360 & 0.0316 & 0.0332 \\
\hline SAABC & $\hat{\mathbf{H}}$ & 0.1968 & 0.4949 & 0.6988 & 0.8954 \\
& $d p$ & 0.0489 & 0.0436 & 0.0408 & 0.0358 \\
& $\sqrt{\mathrm{EQM}}$ & 0.0488 & 0.0438 & 0.0407 & 0.0360 \\
\hline \hline & & & & & \\
& & &
\end{tabular}

Tabela A.3: Resultados obtidos para $H$ através de diferentes métodos em 200 simulações de Monte Carlo do processo binário com memória longa. 


\begin{tabular}{|c|c|c|c|c|c|c|c|}
\hline \multirow[b]{2}{*}{ Método de Estimação } & & \multicolumn{3}{|c|}{$\mathrm{H}=0.6$} & \multicolumn{3}{|c|}{$\mathbf{H}=0.7$} \\
\hline & & $\alpha=1.7$ & $\alpha=1.8$ & $\alpha=1.9$ & $\alpha=1.7$ & $\alpha=1.8$ & $\alpha=1.9$ \\
\hline \multirow[t]{3}{*}{ ECF } & $\hat{\alpha}$ & 1.7080 & 1.8002 & 1.8972 & 1.7083 & 1.7992 & 1.9030 \\
\hline & & 0.0508 & 0.0461 & 0.0399 & 0.0508 & 0.0578 & 0.0453 \\
\hline & $\sqrt{\mathrm{EQM}}$ & 0.0513 & 0.0459 & 0.0399 & 0.0513 & 0.0576 & 0.0453 \\
\hline \multirow{3}{*}{ ABC 2} & $\hat{\alpha}$ & 1.7044 & 1.7952 & 1.8903 & 1.7038 & 1.7935 & 1.8909 \\
\hline & & 0.0511 & 0.0410 & 0.0381 & 0.0475 & 0.0528 & 0.0448 \\
\hline & $\sqrt{\mathrm{EQM}}$ & 0.0512 & 0.0411 & 0.0392 & 0.0476 & 0.0531 & 0.0456 \\
\hline \multirow{2}{*}{\multicolumn{2}{|c|}{ Método de Estimação }} & & $\mathrm{H}=\mathbf{0 . 8}$ & & & $\mathbf{H}=\mathbf{0 . 9}$ & \\
\hline & & $\alpha=1.7$ & $\alpha=1.8$ & $\alpha=\mathbf{1 . 9}$ & $\alpha=1.7$ & $\alpha=1.8$ & $\alpha=\mathbf{1 . 9}$ \\
\hline \multirow[t]{3}{*}{ ECF } & $\hat{\alpha}$ & 1.7192 & 1.8169 & 1.9067 & 1.7240 & 1.8209 & 1.9141 \\
\hline & $d p$ & 0.0685 & 0.0634 & 0.0461 & 0.1050 & 0.0889 & 0.0686 \\
\hline & $\sqrt{\mathrm{EQM}}$ & 0.0710 & 0.0654 & 0.0465 & 0.1075 & 0.0911 & 0.0686 \\
\hline \multirow[t]{3}{*}{ ABC 2} & $\hat{\alpha}$ & 1.7054 & 1.8001 & 1.8825 & 1.7004 & 1.7802 & 1.8720 \\
\hline & $d p$ & 0.0587 & 0.0617 & 0.0441 & 0.0700 & 0.0792 & 0.0592 \\
\hline & $\sqrt{\mathrm{EQM}}$ & 0.0588 & 0.0616 & 0.0474 & 0.0698 & 0.0815 & 0.0653 \\
\hline
\end{tabular}

Tabela A.4: Resultados obtidos para $\alpha$ através dos métodos de Koutrouvelis (1980) e ABC 2 em 200 simulações de Monte Carlo do ruído estável fracionário (fSn). 


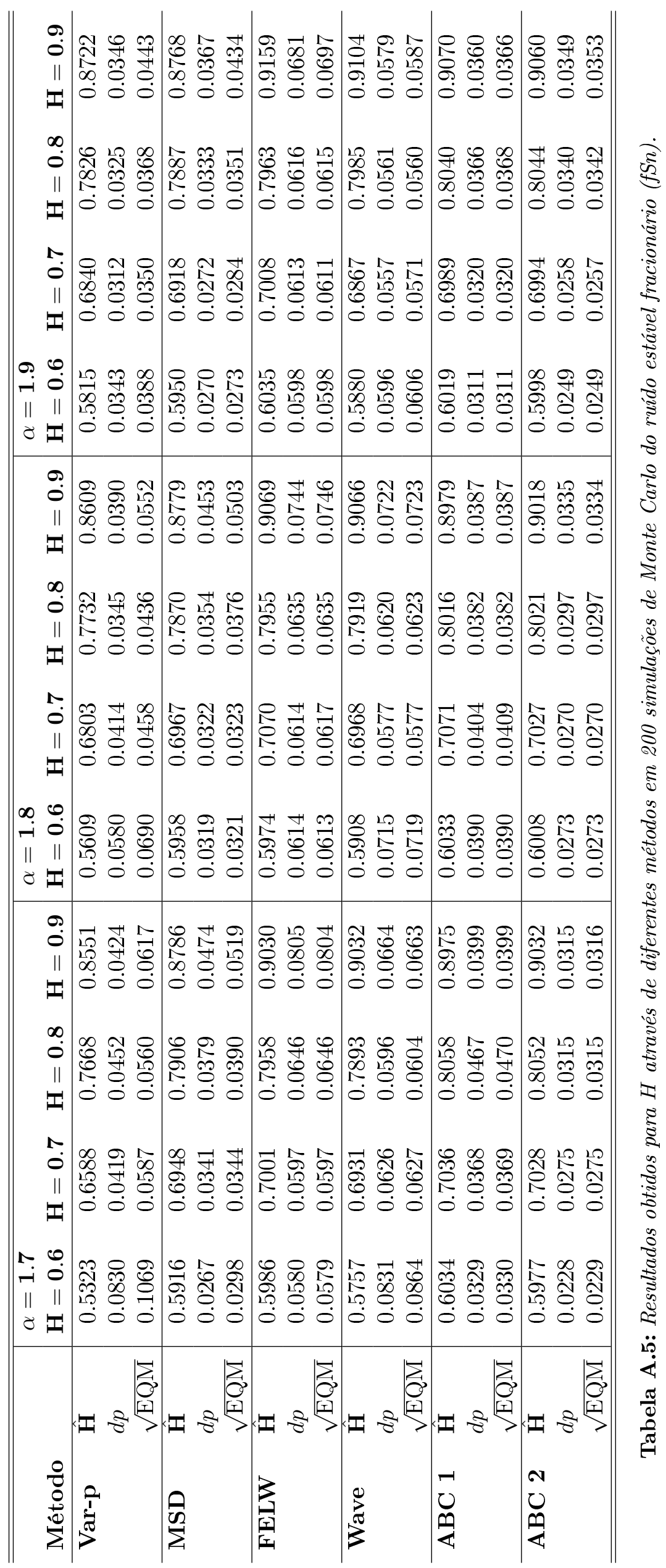




\begin{tabular}{|l|ccc|}
\hline \hline$\theta$ & $\beta=-\mathbf{0 . 9}$ & $\mu=\mathbf{1 0}$ & $\sigma=\mathbf{5}$ \\
\hline$\hat{\theta}$ & -0.8903 & 10.000 & 5.0201 \\
$d p$ & 0.0411 & 0.0006 & 0.2640 \\
$\sqrt{\mathrm{EQM}}$ & 0.0422 & 0.0006 & 0.2640 \\
\hline$\theta$ & $\beta=-\mathbf{0 . 5}$ & $\mu=\mathbf{1 0}$ & $\sigma=\mathbf{5}$ \\
\hline$\hat{\theta}$ & -0.5027 & 9.9997 & 4.9995 \\
$d p$ & 0.0386 & 0.0040 & 0.1754 \\
$\sqrt{\mathrm{EQM}}$ & 0.0386 & 0.0040 & 0.1750 \\
\hline$\theta$ & $\beta=-\mathbf{0 . 1}$ & $\mu=\mathbf{1 0}$ & $\sigma=\mathbf{5}$ \\
\hline$\hat{\theta}$ & -0.1011 & 10.0010 & 4.9929 \\
$d p$ & 0.0316 & 0.0069 & 0.0439 \\
$\sqrt{\mathrm{EQM}}$ & 0.0315 & 0.0069 & 0.0443 \\
\hline$\theta$ & $\beta=\mathbf{0 . 1}$ & $\mu=\mathbf{1 0}$ & $\sigma=\mathbf{5}$ \\
\hline$\hat{\theta}$ & 0.1019 & 10.0011 & 5.0120 \\
$d p$ & 0.0298 & 0.0094 & 0.0363 \\
$\sqrt{\mathrm{EQM}}$ & 0.0298 & 0.0095 & 0.0382 \\
\hline$\theta$ & $\beta=\mathbf{0 . 5}$ & $\mu=\mathbf{1 0}$ & $\sigma=\mathbf{5}$ \\
\hline$\hat{\theta}$ & 0.5031 & 10.0085 & 5.0471 \\
$d p$ & 0.0435 & 0.1140 & 0.1482 \\
$\sqrt{\mathrm{EQM}}$ & 0.0435 & 0.1141 & 0.1552 \\
\hline$\theta$ & $\beta=\mathbf{0 . 9}$ & $\mu=\mathbf{1 0}$ & $\sigma=\mathbf{5}$ \\
\hline$\hat{\theta}$ & 0.8900 & 10.1311 & 5.0842 \\
$d p$ & 0.0466 & 1.7225 & 0.2500 \\
$\sqrt{\mathrm{EQM}}$ & 0.0475 & 1.7231 & 0.2633 \\
\hline \hline
\end{tabular}

Tabela A.6: Resultados obtidos para $(\beta, \mu, \sigma)$ através do método ABC semiautomático em 200 simulações de Monte Carlo dos incrementos do processo gama modulado generalizado, variando $\beta$. 


\section{Referências Bibliográficas}

Abadir et al. (2007) Karim M Abadir, Walter Distaso e Liudas Giraitis. Nonstationarity-extended local whittle estimation. Journal of Econometrics, 141(2):1353-1384. Citado na pág. 29

Abry e Pipiras (2006) Patrice Abry e Vladas Pipiras. Wavelet-based synthesis of the rosenblatt process. Signal processing, 86(9):2326-2339. Citado na pág. 14

Abry et al. (1998) Patrice Abry, Darryl Veitch e Patrick Flandrin. Long-range dependence: Revisiting aggregation with wavelets. Journal of Time Series Analysis, 19(3):253-266. Citado na pág. 29

Allingham et al. (2009) David Allingham, Robert AR King e Kerrie L Mengersen. Bayesian estimation of quantile distributions. Statistics and Computing, 19(2):189-201. Citado na pág. xii, 50,51

Alvarez-Lacalle et al. (2006) Enrique Alvarez-Lacalle, Beate Dorow, Jean-Pierre Eckmann e Elisha Moses. Hierarchical structures induce long-range dynamical correlations in written texts. Proceedings of the National Academy of Sciences, 103(21):7956-7961. Citado na pág. 1

Andrade e Rifo (2015a) Plinio LD Andrade e Laura LR Rifo. Long-range dependence and approximate bayesian computation. Communications in Statistics - Simulation and Computation, (just-accepted). doi: 10.1080/03610918.2014.995816. Citado na pág. 62

Andrade e Rifo (2015b) Plinio LD Andrade e Laura LR Rifo. A note on bayesian inference for long-range dependence of a stationary two-state process. Em Interdisciplinary Bayesian Statistics, páginas 301-310. Springer. Citado na pág. 70

Andrade et al. (2015) Plinio LD Andrade, Laura LR Rifo, Soledad Torres e Francisco TorresAvilés. Bayesian inference on the memory parameter for gamma-modulated regression models. Entropy, 17(10):6576-6597. Citado na pág. xvii, 18, 19, 78, 79, 80, 83, 85

Bardet e Bibi (2012) Jean-Marc Bardet e Hatem Bibi. Adaptive semiparametric wavelet estimator and goodness-of-fit test for long-memory linear processes. Electronic Journal of Statistics, 6:2383-2419. Citado na pág. 30

Bardet e Tudor (2014) Jean-Marc Bardet e Ciprian Tudor. Asymptotic behavior of the whittle estimator for the increments of a rosenblatt process. Journal of Multivariate Analysis, 131:1-16. Citado na pág. 28, 69

Bardet e Tudor (2010) Jean-Marc Bardet e Ciprian Tudor. A wavelet analysis of the rosenblatt process: chaos expansion and estimation of the self-similarity parameter. Stochastic Processes and their Applications, 120(12):2331-2362. Citado na pág. 14

Bardet et al. (2000) Jean-Marc Bardet, G Lang, Eric Moulines e Philippe Soulier. Wavelet estimator of long-range dependent processes. Statistical Inference for Stochastic Processes, 3 (1-2):85-99. Citado na pág. 29 
Bardet et al. (2003) Jean-Marc Bardet, Gabriel Lang, Georges Oppenheim, Anne Philippe e Murad S Taqqu. Generators of long-range dependent processes: a survey. Em Theory and applications of long-range dependence, páginas 579-623. Birkhäuser, Boston, MA. Citado na pág. 12, 13

Bardet et al. (2008) Jean-Marc Bardet, Hatem Bibi e Abdellatif Jouini. Adaptive wavelet-based estimator of the memory parameter for stationary gaussian processes. Bernoulli, 14(3):691-724. Citado na pág. 30

Basu (1975) Debabrata Basu. Statistical information and likelihood. Sankhyā: The Indian Journal of Statistics, Series A, 37(1):1-71. Citado na pág. 53

Beaumont et al. (2002) Mark A Beaumont, Wenyang Zhang e David J Balding. Approximate bayesian computation in population genetics. Genetics, 162(4):2025-2035. Citado na pág. xii, 48, $49,50,51,56$

Benmehdi et al. (2011) S Benmehdi, N Makarava, N Benhamidouche e M Holschneider. Bayesian estimation of the self-similarity exponent of the nile river fluctuation. Nonlinear Processes in Geophysics, 18(3):441-446. Citado na pág. 3

Beran (1994) Jan Beran. Statistics for long memory processes. New York [ua]: Chapman \& Hall. Citado na pág. $2,5,7,11,12,26,29,63,65,76,85$

Beran et al. (2013) Jan Beran, Yuanhua Feng, Sucharita Ghosh e Rafal Kulik. Long-Memory Processes: Probabilistic Properties and Statistical Methods. Springer Science \& Business Media. Citado na pág. $5,24,25,62,85$

Birnbaum (1962) Allan Birnbaum. On the foundations of statistical inference. Journal of the American Statistical Association, 57(298):269-306. Citado na pág. 53

Blum (2010) Michael GB Blum. Choosing the summary statistics and the acceptance rate in approximate bayesian computation. Em Proceedings of COMPSTAT'2010, páginas 47-56. Springer. Citado na pág. 62

Blum e François (2010) Michael GB Blum e Olivier François. Non-linear regression models for approximate bayesian computation. Statistics and Computing, 20(1):63-73. Citado na pág. xii, 49, 50

Blum et al. (2013) Michael GB Blum, Maria Antonieta Nunes, Dennis Prangle, Scott A Sisson et al. A comparative review of dimension reduction methods in approximate bayesian computation. Statistical Science, 28(2):189-208. Citado na pág. 47

Bortot et al. (2007) Paola Bortot, Stuart G Coles e Scott A Sisson. Inference for stereological extremes. Journal of the American Statistical Association, 102(477):84-92. Citado na pág. 33

Brockwell e Davis (2013) Peter J Brockwell e Richard A Davis. Time series: theory and methods. Springer Science \& Business Media. Citado na pág. 12

Burnecki e Weron (2010) Krzysztof Burnecki e Aleksander Weron. Fractional lévy stable motion can model subdiffusive dynamics. Physical Review E, 82(2):021130-1-021130-8. Citado na pág. 31, 32

Burnecki et al. (2008) Krzysztof Burnecki, Joseph Klafter, Marcin Magdziarz e Aleksander Weron. From solar flare time series to fractional dynamics. Physica A: Statistical Mechanics and its Applications, 387(5):1077-1087. Citado na pág. 76, 77 
Burnecki et al. (2012) Krzysztof Burnecki, Marcin Magdziarz e Aleksander Weron. Identification and validation of fractional subdiffusion dynamics. Fractional Dynamics: Recent Advances. J. Klafter, SC Lim, and R. Metzler, editors. World Scientific, New Jersey, páginas 329-349. Citado na pág. 31

Cahoy et al. (2010) Dexter O Cahoy, Vladimir V Uchaikin e Wojbor A Woyczynski. Parameter estimation for fractional poisson processes. Journal of Statistical Planning and Inference, 140 (11):3106-3120. Citado na pág. 85

Chambers et al. (1976) John M Chambers, Colin L Mallows e BW Stuck. A method for simulating stable random variables. Journal of the american statistical association, 71(354):340-344. Citado na pág. 16

Chambers et al. (1987) John M Chambers, Colin L Mallows e BW Stuck. Corrections: A method for simulating stable random variables. Journal of the American Statistical Association, 82(398): 704-704. Citado na pág. 16

Chronopoulou e Viens (2009) Alexandra Chronopoulou e Frederi G Viens. Hurst index estimation for self-similar processes with long-memory. Recent Development in Stochastic Dynamics and Stochastic Analysis, 8:91-117. Citado na pág. 31

Chronopoulou et al. (2008) Alexandra Chronopoulou, Ciprian A Tudor e Frederi G Viens. Selfsimilarity parameter estimation and reproduction property for non-gaussian hermite processes. Communications on Stochastic Analysis, 5(1):161-185. Citado na pág. 31

Chronopoulou et al. (2009) Alexandra Chronopoulou, Frederi G Viens e Ciprian A Tudor. Variations and hurst index estimation for a rosenblatt process using longer filters. Electronic Journal of Statistics, 3:1393-1435. Citado na pág. 31

Cleveland et al. (1990) Robert B Cleveland, William S Cleveland, Jean E McRae e Irma Terpenning. Stl: A seasonal-trend decomposition procedure based on loess. Journal of Official Statistics, $6(1): 3-73$. Citado na pág. 67

Coeurjolly (2000) Jean-François Coeurjolly. Simulation and identification of the fractional brownian motion: a bibliographical and comparative study. J. Stat. Softw., 5(7):1-53. Citado na pág. 13

Cover e Thomas (2006) TM Cover e Joy A Thomas. Elements of information theory. Hoboken, NJ: Wiley-Interscience. Citado na pág. 44

Dahlhaus (1989) Rainer Dahlhaus. Efficient parameter estimation for self-similar processes. The annals of Statistics, páginas 1749-1766. Citado na pág. 23

Didelot et al. (2011) Xavier Didelot, Richard G Everitt, Adam M Johansen e Daniel J Lawson. Likelihood-free estimation of model evidence. Bayesian analysis, 6(1):49-76. Citado na pág. 52

Diggle e Gratton (1984) Peter J Diggle e Richard J Gratton. Monte carlo methods of inference for implicit statistical models. Journal of the Royal Statistical Society. Series B. Methodological, 46(2):193-227. Citado na pág. 34

Dobrushin e Major (1979) Roland Lvovich Dobrushin e Péter Major. Non-central limit theorems for non-linear functional of gaussian fields. Zeitschrift für Wahrscheinlichkeitstheorie und verwandte Gebiete, 50(1):27-52. Citado na pág. 13

Doukhan et al. (2003) Paul Doukhan, George Oppenheim e Murad S Taqqu. Theory and applications of long-range dependence. Springer Science \& Business Media. Citado na pág. 23, 29, 76 
Fearnhead e Prangle (2012) Paul Fearnhead e Dennis Prangle. Constructing summary statistics for approximate bayesian computation: semi-automatic approximate bayesian computation. Journal of the Royal Statistical Society: Series B (Statistical Methodology), 74(3):419-474. Citado na pág. xii, $37,47,49,50,51,53,58$

Fox e Taqqu (1986) Robert Fox e Murad S Taqqu. Large-sample properties of parameter estimates for strongly dependent stationary gaussian time series. The Annals of Statistics, páginas 517-532. Citado na pág. 23, 28

Gamerman e Lopes (2006) Dani Gamerman e Hedibert F Lopes. Markov chain Monte Carlo: stochastic simulation for Bayesian inference. CRC Press. Citado na pág. 33, 39

Giraitis e Surgailis (1990) Liudas Giraitis e D Surgailis. A central limit theorem for quadratic forms in strongly dependent linear variables and its application to asymptotical normality of whittle's estimate. Probability Theory and Related Fields, 86(1):87-104. Citado na pág. 23

Giraitis e Taqqu (1999) Liudas Giraitis e Murad S Taqqu. Whittle estimator for finite-variance non-gaussian time series with long memory. Annals of Statistics, 27:178-203. Citado na pág. 23

Good (1983) Irving John Good. Good thinking: The foundations of probability and its applications. University of Minnesota Press. Citado na pág. 54

Grelaud et al. (2009) Aude Grelaud, Christian P Robert, Jean-Michel Marin, François Rodolphe, Jean-François Taly et al. Abc likelihood-free methods for model choice in gibbs random fields. Bayesian Analysis, 4(2):317-335. Citado na pág. 33, 52, 53

Hamilton (1994) James Douglas Hamilton. Time series analysis, volume 2. Princeton university press. Citado na pág. 26, 42

Haynes et al. (1997) Michele A Haynes, HL MacGillivray e KL Mengersen. Robustness of ranking and selection rules using generalised g-and-k distributions. Journal of Statistical Planning and Inference, 65(1):45-66. Citado na pág. 49

Heyde e Leonenko (2005) Christopher C Heyde e Nikolai N Leonenko. Student processes. Advances in Applied Probability, 37(2):342-365. Citado na pág. 19

Heyde e Yang (1997) Christopher C Heyde e Y Yang. On defining long-range dependence. Journal of Applied Probability, páginas 939-944. Citado na pág. 7

Holan et al. (2009) Scott Holan, Tucker McElroy, Sounak Chakraborty et al. A bayesian approach to estimating the long memory parameter. Bayesian Analysis, 4(1):159-190. Citado na pág. 3

Hurst (1951) Harold Edwin Hurst. Long-term storage capacity of reservoirs. Trans. Amer. Soc. Civil Eng., 116:770-808. Citado na pág. 1, 23, 24, 63

Iglesias et al. (2011) Pilar Iglesias, Jaime San Martín, Soledad Torres e Frederi Viens. Option pricing under a gamma-modulated diffusion process. Annals of Finance, 7(2):199-219. Citado na pág. $18,19,78,82,83$

Jeffreys (1939) Harrold Jeffreys. Theory of Probability. Clarendon Press, Oxford. Citado na pág. 52

Joyce e Marjoram (2008) Paul Joyce e Paul Marjoram. Approximately sufficient statistics and bayesian computation. Statistical Applications in Genetics and Molecular Biology, 7(1):1-18. Citado na pág. $45,46,47$

Karagiannis et al. (2004) Thomas Karagiannis, Mart Molle e Michalis Faloutsos. Long-range dependence ten years of internet traffic modeling. Internet Computing, IEEE, 8(5):57-64. Citado na pág. 1 
Karmeshu e Krishnamachari (2004) Karmeshu e A Krishnamachari. Sequence variability and long-range dependence in dna: An information theoretic perspective. Em NikhilRanjan Pal, Nik Kasabov, RajaniK. Mudi, Srimanta Pal e SwapanKumar Parui, editors, Neural Information Processing, volume 3316 of Lecture Notes in Computer Science, páginas 1354-1361. Springer Berlin Heidelberg. Citado na pág. 1

Kershaw (1983) D Kershaw. Some extensions of w. gautschi's inequalities for the gamma function. Mathematics of Computation, páginas 607-611. Citado na pág. 21

Kotz et al. (2006) Samuel Kotz, Campbell B Read, Narayanaswamy Balakrishnan e Brani Vidakovic. Encyclopedia of Statistical Sciences, volume 12. Wiley Online Library. Citado na pág. 14

Koutrouvelis (1980) Ioannis A Koutrouvelis. Regression-type estimation of the parameters of stable laws. Journal of the American Statistical Association, 75(372):918-928. Citado na pág. xviii, $73,76,77,90$

Leuenberger et al. (2010) Christoph Leuenberger, Daniel Wegmann e Laurent Excoffier. Bayesian computation and model selection in population genetics. Genetics, 184(1):243-252. Citado na pág. 49

Lindley (1957) Dennis V Lindley. A statistical paradox. Biometrika, 44(3):187-192. Citado na pág. 54

Lindley (1997) Dennis V Lindley. Some comments on bayes factors. Journal of Statistical Planning and Inference, 61(1):181-189. Citado na pág. 54

Lo (1997) Aandrew W Lo. Fat tails, long memory, and the stock market since the 1960's'. Economic Notes, 26:219-252. Citado na pág. 1

Magnus et al. (2013) Wilhelm Magnus, Fritz Oberhettinger e Raj Soni. Formulas and theorems for the special functions of mathematical physics, volume 52. Springer Science \& Business Media. Citado na pág. 21

Mandelbrot e Van Ness (1968) Benoit B Mandelbrot e John W Van Ness. Fractional brownian motions, fractional noises and applications. SIAM review, 10(4):422-437. Citado na pág. 1, 8

Mandelbrot e Wallis (1968) Benoit B Mandelbrot e James R Wallis. Noah, joseph, and operational hydrology. Water resources research, 4(5):909-918. Citado na pág. 1, 8

Mandelbrot e Wallis (1969a) Benoit B Mandelbrot e James R Wallis. Robustness of the rescaled range $\mathrm{r} / \mathrm{s}$ in the measurement of noncyclic long run statistical dependence. Water Resources Research, 5(5):967-988. Citado na pág. 1, 8

Mandelbrot e Wallis (1969b) Benoit B Mandelbrot e James R Wallis. Computer experiments with fractional gaussian noises. Water resources research, 5(1):228-267. Citado na pág. 1, 8

Mandelbrot e Wallis (1969c) Benoit B Mandelbrot e James R Wallis. Some long-run properties of geophysical records. Water resources research, 5(2):321-340. Citado na pág. 1, 8

Marin et al. (2012) Jean-Michel Marin, Pierre Pudlo, Christian P Robert e Robin J Ryder. Approximate bayesian computational methods. Statistics and Computing, 22(6):1167-1180. Citado na pág. 37,41

Marjoram et al. (2003) Paul Marjoram, John Molitor, Vincent Plagnol e Simon Tavaré. Markov chain monte carlo without likelihoods. Proceedings of the National Academy of Sciences, 100(26): 15324-15328. Citado na pág. 39 
McCulloch (1986) J Huston McCulloch. Simple consistent estimators of stable distribution parameters. Communications in Statistics-Simulation and Computation, 15(4):1109-1136. Citado na pág. 73

McKinley et al. (2009) Trevelyan McKinley, Alex R Cook e Robert Deardon. Inference in epidemic models without likelihoods. The International Journal of Biostatistics, 5(1). Citado na pág. 33

Moulines e Soulier (1999) Eric Moulines e Philippe Soulier. Broadband log-periodogram regression of time series with long-range dependence. The Annals of Statistics, 27(4):1415-1439. Citado na pág. 26

Oksendal (2013) Bernt Oksendal. Stochastic differential equations: an introduction with applications. Springer Science \& Business Media. Citado na pág. 10

Painter (1998) S Painter. Long-range dependence in the subsurface: Empirical evidence and simulation methods. Em Invited paper at the American Geophysical Union 1998 Fall Meeting. Citado na pág. 1

Peng et al. (1994) C-K Peng, Sergey V Buldyrev, Shlomo Havlin, Michael Simons, H Eugene Stanley e Ary L Goldberger. Mosaic organization of dna nucleotides. Physical Review E, 49(2): 1685-1689. Citado na pág. 27

Pereira e Stern (1999) Carlos A de B Pereira e Julio Michael Stern. Evidence and credibility: full bayesian significance test for precise hypotheses. Entropy, 1(4):99-110. Citado na pág. 53

Pereira et al. (2008) Carlos A de B Pereira, Julio Michael Stern e Sergio Wechsler. Can a significance test be genuinely bayesian? Bayesian Analysis, 3(1):79-100. Citado na pág. 53

Peters et al. (2012) Gareth W Peters, Scott A Sisson e Y Fan. Likelihood-free bayesian inference for $\alpha$-stable models. Computational Statistics 6 S Data Analysis, 56(11):3743-3756. Citado na pág. 73

Pipiras e Taqqu (2010) Vladas Pipiras e Murad S Taqqu. Regularization and integral representations of hermite processes. Statistics 83 probability letters, 80(23):2014-2023. Citado na pág. 11

Pritchard et al. (1999) Jonathan K Pritchard, Mark T Seielstad, Anna Perez-Lezaun e Marcus W Feldman. Population growth of human y chromosomes: a study of y chromosome microsatellites. Molecular biology and evolution, 16(12):1791-1798. Citado na pág. 33, 35

Qu (2005) Leming Qu. Bayesian wavelet estimation of long memory parameter. Journal of Modern Applied Statistical Methods, 4(1):15. Citado na pág. 3

Ratmann et al. (2009) Oliver Ratmann, Christophe Andrieu, Carsten Wiuf e Sylvia Richardson. Model criticism based on likelihood-free inference, with an application to protein network evolution. Proceedings of the National Academy of Sciences, 106(26):10576-10581. Citado na pág. 37

Rayner e MacGillivray (2002) Glen Rayner e Helen MacGillivray. Numerical maximum likelihood estimation for the g-and-k and generalized g-and-h distributions. Statistics and Computing, 12(1):57-75. Citado na pág. 49, 51

Robert (2007) Christian Robert. The Bayesian choice: from decision-theoretic foundations to computational implementation. Springer Science \& Business Media. Citado na pág. 52

Robert e Casella (2013) Christian Robert e George Casella. Monte Carlo statistical methods. Springer Science \& Business Media. Citado na pág. 33, 39 
Robert et al. (2011) Christian P Robert, Jean-Marie Cornuet, Jean-Michel Marin e Natesh S Pillai. Lack of confidence in approximate bayesian computation model choice. Proceedings of the National Academy of Sciences, 108(37):15112-15117. Citado na pág. 53, 54

Robinson (1995) Peter M Robinson. Gaussian semiparametric estimation of long range dependence. Annals of statistics, 23(5):1630-1661. Citado na pág. 29

Robinson (2003) Peter M Robinson. Time series with long memory. Oxford University Press. Citado na pág. 1

Rosenblatt (1961) Murray Rosenblatt. Independence and dependence. Em Proc. 4th Berkeley sympos. math. statist. and prob, volume 2, páginas 431-443. Univ. California Press. Citado na pág. 54

Roueff e Taqqu (2009) François Roueff e Murad S Taqqu. Asymptotic normality of wavelet estimators of the memory parameter for linear processes. Journal of Time Series Analysis, 30 (5):534-558. Citado na pág. 29

Rubin (1984) Donald B Rubin. Bayesianly justifiable and relevant frequency calculations for the applied statistician. The Annals of Statistics, 12(4):1151-1172. Citado na pág. 33, 34

Samorodnitsky (2007) Gennady Samorodnitsky. Long range dependence. Foundations and Trends@ in Stochastic Systems, 1(3):163-257. Citado na pág. 1, 5

Samorodnitsky e Taqqu (1994) Gennady Samorodnitsky e Murad S Taqqu. Stable non-Gaussian random processes: stochastic models with infinite variance, volume 1. CRC Press. Citado na pág. 15, 16

Shafer (1982) Glenn Shafer. Lindley's paradox (with comments). Journal of the American Statistical Association, 77(378):325-351. Citado na pág. 54

Singh et al. (2003) Harshinder Singh, Neeraj Misra, Vladimir Hnizdo, Adam Fedorowicz e Eugene Demchuk. Nearest neighbor estimates of entropy. American journal of mathematical and management sciences, 23(3-4):301-321. Citado na pág. 45

Sottinen (2001) Tommi Sottinen. Fractional brownian motion, random walks and binary market models. Finance and Stochastics, 5(3):343-355. Citado na pág. 13

Stern (2003) Julio Michael Stern. Significance tests, belief calculi, and burden of proof in legal and scientific discourse. Frontiers in Artificial Intelligence and its Applications, 101:139-147. Citado na pág. 54

Stoev e Taqqu (2004) Stilian Stoev e Murad S Taqqu. Simulation methods for linear fractional stable motion and farima using the fast fourier transform. Fractals, 12(01):95-121. Citado na pág. 18

Sun e Cheng (2014) Xichao Sun e Ronglong Cheng. A weak convergence to hermite process by martingale differences. Advances in Mathematical Physics, 2014:1-10. Citado na pág. 14

Taqqu (1978) Murad S Taqqu. A representation for self-similar processes. Stochastic Processes and their Applications, 7(1):55-64. Citado na pág. 13

Taqqu (1979) Murad S Taqqu. Convergence of integrated processes of arbitrary hermite rank. Zeitschrift für Wahrscheinlichkeitstheorie und verwandte Gebiete, 50(1):53-83. Citado na pág. 13

Taqqu (1975) Murad S Taqqu. Weak convergence to fractional brownian motion and to the rosenblatt process. Zeitschrift für Wahrscheinlichkeitstheorie und Verwandte Gebiete, 31(4):287302. Citado na pág. 13,54 
Taqqu (2011) Murad S Taqqu. The rosenblatt process. Em Richard Davis, Keh-Shin Lii e Dimitris Politis, editors, Selected Works of Murray Rosenblatt. Springer Verlag, New York. Citado na pág. 54

Taqqu et al. (1995) Murad S Taqqu, Vadim Teverovsky e Walter Willinger. Estimators for longrange dependence: an empirical study. Fractals, 3(4):785-798. Citado na pág. 23, 28

Tavaré et al. (1997) Simon Tavaré, David J Balding, Robert C Griffiths e Peter Donnelly. Inferring coalescence times from dna sequence data. Genetics, 145(2):505-518. Citado na pág. 33

Toni et al. (2009) Tina Toni, David Welch, Natalja Strelkowa, Andreas Ipsen e Michael PH Stumpf. Approximate bayesian computation scheme for parameter inference and model selection in dynamical systems. Journal of the Royal Society Interface, 6(31):187-202. Citado na pág. 33

Torres e Tudor (2009) Soledad Torres e Ciprian A Tudor. Donsker type theorem for the rosenblatt process and a binary market model. Stochastic Analysis and Applications, 27(3):555-573. Citado na pág. 14

Tudor (2008) Ciprian A Tudor. Analysis of the rosenblatt process. ESAIM: Probability and Statistics, 12:230-257. Citado na pág. 11

Varotsos e Kirk-Davidoff (2006) Costas Varotsos e Daniel Kirk-Davidoff. Long-memory processes in global ozone and temperature variations. Atmospheric Chemistry and Physics Discussions, 6(12):4093-4100. Citado na pág. 1

Veillette et al. (2013) Mark S Veillette, Murad S Taqqu et al. Properties and numerical evaluation of the rosenblatt distribution. Bernoulli, 19(3):982-1005. Citado na pág. 55, 70

Veitch et al. (2003) Darryl Veitch, Patrice Abry e Murad S Taqqu. On the automatic selection of the onset of scaling. Fractals, 11(04):377-390. Citado na pág. 30

Vervaat (1985) Wim Vervaat. Sample path properties of self-similar processes with stationary increments. The Annals of Probability, páginas 1-27. Citado na pág. 9

Vervaat (1987) Wim Vervaat. Properties of general self-similar processes. Katholieke Universiteit Nijmegen. Mathematisch Instituut. Citado na pág. 9

Whittle (1953) Peter Whittle. Estimation and information in stationary time series. Arkiv för matematik, 2(5):423-434. Citado na pág. 28 